\title{
Teoria do momento angular em sistemas complexos
}

Tese apresentada à Faculdade de Filosofia, Ciências e Letras de Ribeirão Preto da Universidade de São Paulo como parte das exigências para a obtenção do título de Doutor em Ciências.

Área de Concentração:

Física Aplicada à Medicina e Biologia.

Orientador:

Alexandre Souto Martinez. 
Autorizo a reprodução e divulgação total ou parcial deste trabalho, por qualquer meio convencional ou eletrônico, para fins de estudo e pesquisa, desde que citada a fonte.

FICHA CATALOGRÁFICA

Nakamura, Gilberto Medeiros

Teoria do momento angular em sistemas complexos / Gilberto Medeiros

Nakamura; orientador: Alexandre Souto Martinez. - - Ribeirão Preto, 2017. $92 \mathrm{f}$ : : il.

Tese (Doutorado) - - Faculdade de Filosofia, Ciências e Letras de Ribeirão Preto, Universidade de São Paulo, 2017.

Inclui Bibliografia.

1. Física estatística 2. Modelos epidêmicos 3. Processos estocásticos 4. Transições de fase 
Nome: Nakamura, Gilberto Medeiros

Título: Teoria do momento angular em sistemas complexos

Tese apresentada à Faculdade de Filosofia, Ciências e Letras de Ribeirão Preto da Universidade de São Paulo como parte das exigências para a obtenção do título de Doutor em Ciências.

Aprovado em:

\section{Banca Examinadora}

$\operatorname{Prof}(\mathrm{a}) \cdot \operatorname{Dr}(\mathrm{a})$.

Instituição:

Julgamento:

Assinatura:

$\operatorname{Prof}(\mathrm{a}) \cdot \operatorname{Dr}(\mathrm{a})$.

Instituição:

Julgamento:

Assinatura:

Prof(a). $\operatorname{Dr}(\mathrm{a})$.

Instituição:

Julgamento:

Assinatura:

Prof(a). $\operatorname{Dr}(\mathrm{a})$.

Instituição:

Julgamento:

Assinatura:

$\operatorname{Prof}(\mathrm{a}) . \operatorname{Dr}(\mathrm{a})$.

Instituição:

Julgamento:

Assinatura: 


\section{Resumo}

NAKAMURA, G. M. Teoria do momento angular em sistemas complexos. 2017. $92 \mathrm{f}$. Tese (Doutorado - Programa de Pós-Graduação em Física Aplicada à Medicina e Biologia) - Faculdade de Filosofia, Ciências e Letras de Ribeirão Preto, Universidade de São Paulo, Ribeirão Preto, 2017.

A emergência de fenômenos coletivos e correlações de longo alcance impossibilitam a inferência de propriedades de sistemas como um todo a partir de suas partes componentes. A modelagem destes sistemas frequentemente ocorre mediante emprego de operadores de spin localizados em grafos com topologias não-triviais. Aqui, mostramos que o operador de momento angular de muitos corpos une o estudo de diversos sistemas complexos, desde a sistemas epidêmicos até cadeias magnéticas de spin. Para o modelo epidêmico SIS, determinamos a matriz de transição do processo estocástico correspondente e mostramos suas soluções para grafos regulares e aleatórios, por meio de técnicas geralmente empregadas em sistemas fortemente correlacionados. Já no modelo de Dicke, identificamos o vínculo que explica a relevância e o efeito finito de operadores anti-girantes para duas espécies atômicas confinadas numa cavidade óptica que interagem com radiação eletromagnética. Por fim, o papel do momento angular também é identificado para duas cadeias quânticas de spin $1 / 2$ acopladas, as quais modelam nanoestruturas magnéticas heterogêneas. A estrutura de bandas é calculada, enquanto efeitos espúrios de superfície são removidos pela introdução de quasipartículas dotadas de grau de liberdade de spin adicional.

Palavras-chave: 1. Física estatística 2. Modelos epidêmicos 3. Processos estocásticos 4. Transições de fase 


\section{Abstract}

NAKAMURA, G. M. Theory of angular momentum in complex systems. 2017. $92 \mathrm{f}$. Thesis (Ph.D. - Postgraduate program in Physics Applied to Medicine and Biology) - Faculty of Philosophy, Sciences and Letters, University of São Paulo, Ribeirão Preto, 2017.

The emergence of collective phenomena and long range correlations makes it impossible to infer the properties of whole systems from their components. Their modeling often occurs through the use of localized spin operators, taking place within graphs with non-trivial topologies. Here, we show that the many-body angular momentum operator connects the study of several complex systems, ranging from epidemic systems to magnetic spinchains. For the SIS epidemic model, we calculate the transition matrix of the corresponding stochastic process and show the corresponding solutions for regular and random graphs, using techniques generally employed in strongly correlated systems. For the Dicke model we identify the constraint that explains the relevance and finite size effect of anti-rotating operators, for two atomic species, confined within an optical cavity, and interacting with electromagnetic radiation. Finally, the role of angular momentum is also identified for two coupled quantum spinchains $1 / 2$ which model heterogeneous magnetic nanostructures. The band structure is calculated, while spurious surface effects are removed due to the introduction of quasiparticles with an additional spin degree of freedom.

Key-words: 1. Statistical Physics 2. Epidemic models 3. Stochastic processes 4. Phase transitions 


\section{Sumário}

1 Introdução 1

2 Momento angular 5

2.1 Spin . . . . . . . . . . . . . . . . 8

2.2 Operadores de spin localizados . . . . . . . . . . . . . 12

2.3 Fermionização . . . . . . . . . . . . . . . . . . 15

2.4 Fermionização: aplicação no modelo XY . . . . . . . . . . . . . 18

2.5 Bosonificação . . . . . . . . . . . . . . . . . . . . . . . 22

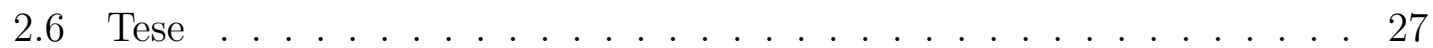

3 Aplicações em modelos epidemiológicos $\quad 29$

3.1 Modelos epidêmicos . . . . . . . . . . . . . . . . . 34

3.2 Abordagem determinística compartimental . . . . . . . . . . . . . . 38

3.3 Abordagem estocástica baseada em agentes . . . . . . . . . . . . . . . 43

3.4 Operadores e a matriz de transição . . . . . . . . . . . . . . . . . . 49

3.5 Observáveis e evolução temporal . . . . . . . . . . . . . . . . 57

3.6 Norma do vetor probabilidade . . . . . . . . . . . . . . . 65

3.7 Teoria de Perturbação . . . . . . . . . . . . . . . . . 74

3.7.1 Redes aleatórias . . . . . . . . . . . . . 74

3.7 .2 Redes periódicas . . . . . . . . . . . . 77

3.8 Outras aplicações epidemiológicas . . . . . . . . . . . . . . . . . 79

3.9 Conclusão . . . . . . . . . . . . . . . . . . . . . . . . 83

4 Simetrias em cavidades ópticas $\quad 85$

4.1 Modelo de Dicke . . . . . . . . . . . . . . . . . . . . . . . . 86

4.2 Efeitos de $N$ finito . . . . . . . . . . . . . . . . . . . . . . 90 
4.3 Simetria $U(1) \ldots \ldots \ldots 5$

4.4 Operadores angulares . . . . . . . . . . . . . . . 100

4.5 Duas espécies atômicas . . . . . . . . . . . . . . . . 101

4.6 Conclusão . . . . . . . . . . . . . . . . . . . . . . . . . . 104

5 Nanoestruturas magnéticas $\quad 107$

5.1 Cadeias de spin . . . . . . . . . . . . . . . . . . 111

5.2 Fermionização . . . . . . . . . . . . . . . . . . . 113

5.3 Modelo . . . . . . . . . . . . . . . . . . . . 115

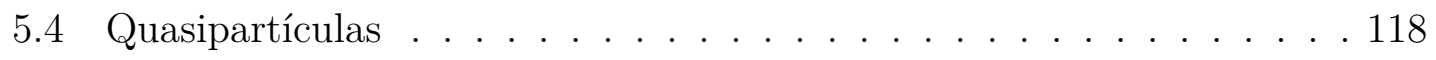

5.5 Hamiltoniano canônico . . . . . . . . . . . . . . . . . . . . . . . . 122

5.6 Conclusão . . . . . . . . . . . . . . . . . . . . . . 125

6 Conclusão e perspectivas $\quad 127$

Apêndice 1 - Grafos e redes 131

1.1 Redes . . . . . . . . . . . . . . . . . . . . . . 133

1.2 Combinação linear de Grafos . . . . . . . . . . . . . . . . . . 136

Apêndice 2 - Simetrias finitas e implementação computacional $\quad 139$

2.1 Espaço vetorial . . . . . . . . . . . . . . . . . . . . . . . 139

2.2 Representação matricial . . . . . . . . . . . . . . . . . . . . . . . 143

Apêndice 3 - Matriz de transição para mais que dois estados epidêmi$\begin{array}{ll}\cos & 147\end{array}$

3.1 Matrizes de Weyl . . . . . . . . . . . . . . . . . . . . . . 147

3.2 Movimentos difusivos de patógenos . . . . . . . . . . . . . . . . . 148

3.3 Endemias . . . . . . . . . . . . . . . . . . . . . . . . 149

Apêndice 4 - Aproximações $\quad 153$

4.1 Estados coerentes . . . . . . . . . . . . . . . . . . . 153

4.2 Aproximação de Bethe-Peierls . . . . . . . . . . . . . . . . . 156

Apêndice 5 - Aplicações em processos estocásticos com memória $\quad 157$

$\begin{array}{ll}\text { Referências } & 161\end{array}$ 


\section{Introdução}

Nessa época de especialização, homens que meticulosamente conhecem uma área são incompetentes para discutir outra.

- Richard Feynman

O estudo das interações entre agentes, partículas e campos é crucial para o entendimento correto de qualquer teoria física. A partir das interações compreendese o relacionamento entre as diferentes partes que compõem um sistema e calcula-se grandezas físicas relevantes para a previsão de seu comportamento ou propriedades físico-químicas. Em geral, tais cálculos são realizados sobre um pequeno subconjunto do sistema donde as conclusões são extrapoladas para toda a extensão do sistema. Por exemplo, a condutividade elétrica de uma placa de cobre de $400 \mathrm{~m}^{2}$, à temperatura ambiente, pode ser inferida a partir de uma pequena ${ }^{1}$ amostra de apenas $1 \mathrm{~cm}^{2}$. Sistemas que se comportam desta maneira são denominados de normais, e compõem a enorme maioria dos sistemas físicos existentes.

Nas últimas décadas, uma classe de sistemas que se comporta de modo anômalo tem atraído atenção da comunidade científica. Essa classe foi cunhada pelo termo sistemas complexos. Exemplos são facilmente encontrados nas mais variadas áreas do conhecimento: epidemias de doenças comunicáveis ou vírus computacionais em redes livre de escala [1]; análise de risco para mercado financeiro [2, 3]; estrutura funcional do cérebro humano [4]; cadeias de spin com acoplamentos aleatórios [5] e outros sistemas fortemente correlacionados [6-9], entre outros. Nos sistemas

\footnotetext{
${ }^{1} \mathrm{O}$ critério requerido para se determinar o quão pequeno é a "pequena amostra" repousa sobre o comprimento de correlação, $\xi$. Se $x(\vec{r})$ é a variável localizada na posição $\vec{r}$, a função de correlação $\langle x(\vec{r}) x(\overrightarrow{0})\rangle \sim \exp (-|\vec{r}| / \xi)$ decai exponencialmente com a escala determinada por $\xi$. Uma amostra é representativa de um sistema normal se suas dimensões lineares $l$ satisfazem $l \gg \xi$.
} 
complexos, as predições de propriedades globais baseadas em padrões locais não se realizam: as interações produzem correlações de longo alcance entre os vários entes componentes do sistema. Equivalentemente, observa-se a emergência de comportamentos e padrões coletivos, em contraposição às interações locais e simples. Num linguajar mais familiar, modos normais de baixa frequência - de extensão da ordem do tamanho do sistema - adquirem maior relevância na descrição das propriedades físicas frente aos modos normais de alta frequência.

A investigação de sistemas complexos tem criado novos métodos e perspectivas nas áreas de conhecimento correspondentes. Tais desenvolvimentos ocorrem de maneira paralela e independente. Não há, em princípio nenhuma motivação para utilizá-las em contextos diferentes daquele de origem. Em áreas mais tradicionais da Física de estado sólido, técnicas como a fermionização [10] ou bosonificação [11] de operadores, além das transformações de Bogoliubov [12], são ferramentas corriqueiras. Já a modelagem epidemiológica baseada em agentes [13] faz uso extensivo de grafos e redes [14] como mecanismo descritor da heterogeneidade das relações sociais dos agentes.

Apesar das diferentes naturezas físicas, matemáticas ou biológicas, observa-se grande similaridade nas ferramentas matemáticas empregadas no modelamento de sistemas complexos. Mais precisamente, as interações são derivadas a partir de operadores localizados, cujas relações algébricas [15] são aquelas dos operadores de spin. Apesar de sua simplicidade, modelos de spins são notórios por gerar comprimentos de correlação $\xi$ da ordem do tamanho do sistema, muito embora as interações sejam de curto alcance $[16,17]$. O exemplo físico mais famoso é o modelo de Ising [18], que descreve um mecanismo rudimentar para o surgimento da magnetização espontânea de meios magnéticos. Adicionalmente, vizinhanças não-triviais para a topologia das interações introduzem elementos que muitas vezes contrapõem-se às expectativas derivadas a partir de padrões regulares.

A discussão que permeia fenômenos coletivos e operadores localizados de spin, presentes em sistemas complexos, converge naturalmente para o estudo da teoria de momento angular de muitos corpos [19]. Sob essa óptica, colocamos adiante a seguinte tese: a teoria do momento angular provê a metodologia adequada para análises de variadas classes de sistemas complexos, unificadas sob uma mesma 
perspectiva. É nesse contexto que este estudo se insere, apropriadamente detalhado no Capítulo 2. Para comprovar ou rejeitar a tese proposta, realizou-se o estudo de três sistemas complexos: modelo epidêmico, modelo de Dicke com duas espécies atômicas e nanoestruturas de spin $1 / 2$ acopladas.

Os processos estocásticos [20] de modelos epidêmicos em grafos e redes são tratados extensivamente no Capítulo 3. Apesar de serem bem conhecidos, as abordagens Markovianas não gozam ampla difusão em Epidemiologia [21]. Em parte isso se deve ao abrupto incremento exponencial do esforço computacional e teórico necessário para o cálculo de grandezas relevantes. Entretanto, conforme descrevem os autores na Ref. [22], faz-se mister a adoção de métodos estocásticos para o modelamento adequado da dinâmica de propagação de doenças comunicáveis de baixa difusão como sarampo, e novas infecções emergentes como pneumonia atípica (SARS) ou síndrome respiratória do Oriente Médio (MERS). Dentre os resultados obtidos, e possivelmente o mais expressivo, mostramos que a mera descrição do modelo epidêmico SIS [23] em termos de operadores de momento angular permite reduzir a complexidade do problema de $O\left(2^{N}\right)$ para $O(N)$. Mais ainda, permite também a incorporação de métodos provenientes de sistemas fortemente correlacionados à Epidemiologia e, por conseguinte, a consideração de flutuações, outrora negligenciadas.

Os estudos pertinentes ao Capítulo 4 focam atenção no modelo de Dicke [24-26] para dois ensembles formados por duas espécies atômicas, confinadas numa cavidade óptica, e que interagem com radiação eletromagnética. Grosso modo, o modelo de Dicke descreve a interação entre radiação e matéria, esta última representada de modo simplificado por um conjunto de átomos de dois níveis. Um dos fenômenos interessantes descrito por esse modelo é a superradiância [27-29], que é a emissão espontânea de radiação eletromagnética por um ensemble atômico, de modo coletivo. Neste fenômeno, os átomos aprisionados na cavidade são mantidos nos respectivos estados excitados por longos intervalos de tempo. Apesar da interação interatômica ser omitida no modelo de Dicke, a cavidade introduz vínculos para o campo eletromagnético confinado, compartilhado por todos os átomos. Assim, o decaimento espontâneo de um único átomo induz todos os outros átomos a decaírem para seus estados fundamentais, produzindo um pulso intenso de curta 
duração. Ao explorar as leis de conservação do momento angular e métodos como a transformação Holstein-Primakoff, mostramos que os operadores contragirantes, geralmente negligenciados, desempenham papel fundamental tanto na interpretação de conservação de energia quanto na transição superradiante.

Por fim, a descrição de uma nanoestrutura magnética formada por duas cadeias de spin $1 / 2$ fermiônicas é estudada em detalhes no Capítulo 5. Cadeias quânticas de spin são relevantes para uma grande gama de problemas, desde materiais magnéticos [30] até processos estocásticos de reação-difusão [31]. Constante em todos os fenômenos aqui investigados é a presença de fenômenos coletivos e transições de fase contínua [32, 33]. A finitude da cadeia introduz correções não-triviais para seu espectro de excitação, a partir das quais seu comportamento crítico é extraído mediante invariância conforme [32, 34-37]. Como cadeias finitas se encontram no mesmo domínio que nanomateriais, investigamos as propriedades de cadeias de spin $1 / 2$ interagentes. Em particular, observa-se o fenômeno de corrente de spin devido à interação entre as cadeias. Neste caso, as relações algébricas observadas pelos operadores do modelo são interpretadas como àquelas do momento angular deformado. Isto permite a identificação de quasipartículas dotadas de grau de liberdade de spin e a determinação aproximada da estrutura de bandas do modelo.

O Capítulo 6 apresenta as considerações finais sobre as aplicações da teoria do momento angular e de muitos corpos em sistemas físicos e processos estocásticos, bem como perspectivas futuras. Os apêndices abordam matérias importantes para o desenvolvimento do capítulos, como a implementação numérica da base invariante por permutações cíclicas, por exemplo. Por fim, apesar da extensão destes estudos, nem todos os assuntos tratados ao longo destes dois anos foram documentados aqui. 


\section{2}

\section{Momento angular}

A Natureza não estava satisfeita com uma simples carga putual mas exigiu uma carga com spin.

- Sin-Itiro Tomonaga

O momento angular é uma das primeiras grandezas físicas apresentadas em cursos introdutórios de Mecânica [38]. A forma mais simples e talvez mais intuitiva de descrevê-lo é a partir da isotropia espacial [39]. Um sistema é isotrópico quando suas propriedades físicas não se alteram nas diferentes direções espaciais, o que equivale a dizer que elas não se alteram mediante rotações espaciais, dado um eixo de referência. Em Mecânica Clássica, a grandeza que exprime tal conservação e que caracteriza o sistema é nada mais que o vetor momento angular $\vec{L}=\vec{r} \times \vec{p}$, onde $\vec{r}$ é o vetor posição e $\vec{p}$ é o momento linear. Os efeitos do momento angular são bastante evidentes nas atividades do dia-a-dia, no movimento de um pião, nos giros de uma bailarina, nas órbitas dos planetas, etc. Apesar de sua origem clássica, o momento angular não é restrito apenas aos domínios da Física Clássica, sendo igualmente fundamental para a formulação de teorias Quânticas [40, 41]. Concomitante ao momento angular, seja ele descrito por teorias clássicas ou não, caminha o conceito de simetrias por rotações [42], dessa vez não necessariamente espaciais. Desse modo, antes de adentrar à temática central desta tese, convém dissertar sobre os conceitos de simetrias e transformações, os quais provêm o suporte necessário aos métodos desenvolvidos aqui.

Formalmente, um grupo de transformações, ou simplesmente grupo, é uma estrutura formada por um conjunto de transformações $\left\{e_{0}, e_{1}, \ldots\right\}$, as quais se re- 
lacionam entre si por meio de uma operação [43]. Mais precisamente, a operação entre dois elementos quaisquer do grupo sempre produz um terceiro elemento, também pertencente ao grupo, propriedade conhecida como fechamento. Devido à essa definição, a transformação identidade $e_{0}$ é comum a qualquer grupo. A definição exata da operação depende do grupo. No caso do grupo das transformações geométricas, a operação é o produto, muito embora o tipo específico do produto dependa da representação das transformações. Por exemplo, se as transformações são representadas por números complexos, a operação é o produto entre números complexos; se as transformações são representadas por meio de matrizes, trata-se do produto matricial.

Uma descrição muito mais intuitiva de grupos lida com a classificação funcional do conjunto das transformações $\left\{e_{0}, e_{1}, \ldots\right\}$. Por exemplo, o conjunto formado por todas as translações espaciais ou mesmo todas as rotações espaciais. Há, portanto, um mote comum à formação de cada grupo, permitindo sua classificação de acordo com sua ação: grupos de todas as transformações geométricas, apenas de rotações ou translações, inversões, etc. Existem outras características classificadoras como, por exemplo, o número de elementos componentes do grupo. Neste caso, subdividem-se em grupos finitos ou contínuos, o primeiro quando o número de elementos é finito e o segundo, caso contrário. Os grupos contínuos, mais conhecidos como grupos de Lie [15], compreendem as rotações e são essenciais para a formulação e caracterização do momento angular na teoria quântica.

Diz-se que um sistema é invariante ou simétrico pelo grupo $G$ se a ação de qualquer elemento $g_{k} \in G(k=0,1, \ldots)$ sobre o sistema mantiver suas propriedades físicas inalteradas. O campo elétrico gerado por uma carga elétrica puntual $q$ possui simetria esférica (invariância por rotações espaciais), cuja manifestação é observada pela ausência de dependências angular. A conexão explícita entre simetrias e grandezas físicas jaz num dos teoremas mais notáveis da Física: o teorema de Noether, derivado em 1918 [44, 45]. Grosso modo, o teorema é assim enunciado: para cada simetria há uma lei de conservação. Na Mecânica Clássica, as leis de conservação correspondem às constantes de movimento [38], ingredientes essenciais para a descrição apropriada das equações de movimento de partículas. Um exemplo bem conhecido é a conservação do momento linear para sistemas invariantes por 
translação, com homogeneidade espacial [39]. Já nas teorias quânticas, as leis de conservação resultam em degenerescências no espectro de energia [41]. Assim, de maneira singela, a explicação de fenômenos físicos passa também a contemplar a investigação de simetrias e seus efeitos correspondentes nas equações de movimentos, campos, espectro de energias e demais propriedades.

De acordo com o teorema de Noether, as leis de conservação do momento angular decorrem da simetria por rotações, tanto na Mecânica Clássica quanto na Quântica. No caso específico das teorias quânticas, a conservação do momento angular adquire significado de números quânticos, os quais atuam como elementos classificadores ou agrupadores de acordo com o estado físico do sistema. Por exemplo, os níveis $s, p, d$ e $f$ do diagrama de Pauling são bons números quânticos para átomos hidrogenóides. As rotações ainda podem ser subdivididas em duas grandes classes: as rotações espaciais, associadas aos operador de momento angular orbital $\hat{L}_{\alpha}(\alpha=x, y, z)$; e as rotações intrínsecas das partículas, que correspondem aos operadores de spin $\hat{S}_{\alpha}(\alpha=x, y, z) \cdot{ }^{1}$ O momento angular total $\hat{J}_{\alpha}=\hat{L}_{\alpha}+\hat{S}_{\alpha}$ é nada mais que a resultante entre o momento angular orbital e o spin.

A partir da rotação infinitesimal $\hat{R}_{\alpha}(\delta \theta)=\mathbb{1}-\imath(\delta \theta / \hbar) \hat{J}_{\alpha}$, por um ângulo infinitesimal $\delta \theta$ em torno do eixo $\alpha$, obtém-se

$$
\hat{R}_{\alpha}(\theta)=\mathrm{e}^{-\imath \theta \hat{J}_{\alpha} / \hbar}
$$

A simplicidade da forma exponencial presente na Eq. (2.1) vale para qualquer grupo de Lie [15], propriedade esta que é rastreada à álgebra de Lie correspondente. No caso das rotações, os geradores da álgebra de Lie são proporcionais aos operadores de momento angular $\hat{J}_{\alpha}(\alpha=x, y, z)$. Uma álgebra de Lie é caracterizada pelo número de geradores e também por suas constantes de estruturas (relações de comutação), que são

$$
\left[\hat{J}_{\alpha}, \hat{J}_{\beta}\right]=\imath \hbar \epsilon_{\alpha \beta \gamma} \hat{J}_{\gamma}
$$

onde $\epsilon_{\alpha \beta \gamma}$ é o tensor totalmente anti-simétrico de Levi-Civitta. As combinações dos

\footnotetext{
${ }^{1}$ Seguindo a convenção da Ref. [42], adotamos o acento circunflexo para diferenciar operadores de números complexos. Embora essa convenção não seja muito usual, foi um hábito que adquiri ao longo dos cursos de Física Matemática e Teoria de Grupos, que pouco a pouco se tornou vício de linguagem. À época, a razão que me convenceu a adotar essa convenção foi a sobrevivência de um astronauta fictício, cuja vida era significativamente sujeita aos erros de alunos de Física Matemática.
} 
geradores $\hat{J}_{x}$ e $\hat{J}_{y}$ expressam os operadores de levantamento $\hat{J}_{+}$e abaixamento $\hat{J}_{-}$,

$$
\begin{aligned}
& \hat{J}_{+}=\hat{J}_{x}+\imath \hat{J}_{y}, \\
& \hat{J}_{-}=\hat{J}_{x}-\imath \hat{J}_{y} .
\end{aligned}
$$

Os operadores $\hat{J}_{ \pm, z}$ satisfazem as seguintes relações de comutação

$$
\begin{aligned}
& {\left[\hat{J}_{z}, \hat{J}_{ \pm}\right]= \pm \hbar \hat{J}_{ \pm},} \\
& {\left[\hat{J}_{+}, \hat{J}_{-}\right]=2 \hbar \hat{J}_{z} .}
\end{aligned}
$$

A outra propriedade relevante das álgebras de Lie decorre da existência de uma álgebra envelope, mais especificamente, o operador de Casimir quadrático $\hat{J}^{2}$. Por definição, o operador de Casimir comuta com todos os geradores,

$$
\left[\hat{J}_{\alpha}, \hat{J}^{2}\right]=0
$$

para $\alpha= \pm, x, y, z$. Por este motivo, o operador de Casimir produz uma quantidade conservada (número quântico) que caracteriza o sistema. No caso das rotações, $\hat{J}^{2}$ refere-se ao momento angular total cujos autovalores são $\hbar^{2} j(j+1)$, com $j$ inteiro para bóson e semi-inteiro para férmions [40].

\subsection{Spin}

A introdução conceitual do spin como grau de liberdade intrínseca de partículas elementares ocorreu em 1926, por Uhlenbeck e Goudsmith [46]. ${ }^{2}$ Em seu artigo original, com pouco mais de uma página e meia, e com uma breve nota de recomendação de Niels Bohr, a dupla propõe o spin como alternativa para explicar propriedades espectrais de certos átomos sujeitos à ação de campos magnéticos, o efeito Zeeman anômalo. Esse fenômeno já tivera sido previamente estudado por Pauli $^{3}$, e o levara a propor um quarto número quântico. A proposta de Pauli foi, por sua vez, influenciada pelas evidências empíricas da teoria de estabilidade molecular de Bohr-Sommerfeld para orbitais moleculares fechados. No entanto, coube a dupla

\footnotetext{
2 À época, Uhlenbeck possuía apenas graduação e Goudsmith, título de mestre, em Leiden. A explicação desenvolvida passou pelo crivo de cientistas renomados como Lorentz, Ehrenfest e Bohr. Este último encarregou-se de promover a ideia para Heisenberg, Pauli e Einstein [47].

${ }^{3}$ Assim como Bohr e Ehrenfest, Pauli também teve papel ímpar no desenvolvimento nos primórdios da formulação da teoria quântica. Neste caso, negativamente. De acordo com a Ref. [47], Kronig revela a Kramers que havia proposto a ideia do spin em 1925, utilizando argumentos semelhantes aos de Uhlenbeck e Goudsmith. A publicação de sua ideia não foi adiante após ser ridicularizada por Pauli.
} 
de cientistas holandeses associar a existência de um número quântico com um grau de liberdade adicional para os elétrons.

O experimento de Stern-Gerlach (1922) produz evidências empíricas bastante contundentes sobre a existência do spin. O experimento, presente em qualquer laboratório de ensino de ciências físicas, é bastante simples: um forno emite um feixe de átomos de prata, eletricamente neutros, os quais são então sujeitos à ação de um campo magnético axial mas não-uniforme. ${ }^{4}$ Ao viajar pela região imersa no campo magnético, as partículas presentes no feixe experimentam forças que alteram suas trajetórias, como esperado. Porém, o experimento revela a existência de apenas dois feixes resultantes após a interação com o campo magnético, dispostos ao longo da direção deste último. O comportamento clássico esperado era que o feixe se abrisse continuamente ao longo de uma linha, assumindo-se a distribuição aleatória da componente axial do momento magnético dos átomos. A repetição do experimento, dessa vez com o eixo do campo magnético rotacionado, novamente produziu apenas dois feixes. Verificou-se que as propriedades físicas dos feixes resultantes permaneciam as mesmas salvo pela interação magnética.

A interação dipolar magnética entre os átomos e o campo magnético introduz uma direção preferencial no espaço, ou seja, resulta na anisotropia espacial e, portanto, na quebra de simetria rotacional. Mais ainda, há independência da duplicidade da função de onda correspondente aos átomos Ag (feixes) quanto à rotações arbitrárias do eixo do campo magnético. Segue que a função de onda das partículas antes da interação com o campo é a combinação linear de duas funções de onda, estas últimas dependentes da direção do eixo do campo magnético. Ora, tal problema já havia sido extensivamente estudado apenas alguns anos antes na descrição espectral do átomo $\mathrm{H}$, onde uma única função de onda poderia ser decomposta como combinação linear de $2 l+1$ outras funções de base, cada uma identificada pelo número quântico do momento angular orbital discretizado. No caso do átomo Ag, a existência de dois feixes decorria de um único elétron. Segue daí duas conclusões importantes: o spin eletrônico é $s=1 / 2$ e essa grandeza física se comporta da mesma maneira que o momento angular. Em linhas mais gerais, os operadores de

\footnotetext{
${ }^{4}$ Convém estabelecer a não-uniformidade do campo magnético pois a força oriunda da interação deste com o dipolo magnético é $\vec{F}=-\vec{\nabla}(\vec{\mu} \cdot \vec{B})$, onde $\vec{\mu}$ é o momento magnético. Logo, campos uniformes iriam produzir força apenas quando o dipolo adentrasse ou saísse à região com campo magnético constante, produzindo efeitos de difícil observação.
} 
spin também são geradores de rotação assim como o momento angular orbital.

$\mathrm{Na}$ versão não-relativística, ${ }^{5}$ o spin eletrônico para o elétron imerso num campo eletromagnético é introduzido na equação de Schrödinger pelo seguinte Hamiltoniano:

$$
\hat{H}=\frac{1}{2 m_{e}}\left[\hat{\boldsymbol{\sigma}} \cdot\left(\hat{\boldsymbol{p}}-\frac{e}{c} \hat{\boldsymbol{A}}\right)\right]^{2}+e \hat{\varphi}
$$

onde $e$ é a carga elétrica, $c$ é a velocidade da luz, $\hat{\boldsymbol{p}}=\left(\hat{p}_{x}, \hat{p}_{y}, \hat{p}_{z}\right)$ são os operadores de momento linear, $\hat{\boldsymbol{A}}=\left(\hat{A}_{x}, \hat{A}_{y}, \hat{A}_{z}\right)$ é o potencial vetor, $\hat{\phi}$ é o potencial escalar e $\hat{\boldsymbol{\sigma}}=\left(\hat{\sigma}_{x}, \hat{\sigma}_{y}, \hat{\sigma}_{z}\right)$ são as matrizes de Pauli. As matrizes de Pauli são melhor apreciadas na base $|\uparrow\rangle$ e $|\downarrow\rangle$,

$$
\hat{\sigma}_{x}=\left(\begin{array}{ll}
0 & 1 \\
1 & 0
\end{array}\right), \quad \hat{\sigma}_{y}=\left(\begin{array}{rr}
0 & -\imath \\
\imath & 0
\end{array}\right), \quad \hat{\sigma}_{z}=\left(\begin{array}{rr}
1 & 0 \\
0 & -1
\end{array}\right) .
$$

Elas possuem relações algébricas muito bem definidas, compatíveis com aquelas da álgebra $s u(2):\left[\hat{\sigma}_{\mu}, \hat{\sigma}_{\nu}\right]=2 \imath \epsilon_{\mu \nu \rho} \hat{\sigma}_{\rho}$, com $\epsilon_{\mu \nu \rho}$ sendo o tensor completamente antisimétrico de Levi-Civitta. A compatibilidade torna-se igualdade mediante a identificação dos operadores de spin $\hat{S}_{\mu}=(\hbar / 2) \hat{\sigma}_{\mu}$. Além disso, satisfazem também a identidade $(\hat{\boldsymbol{\sigma}} \cdot \boldsymbol{a})(\hat{\boldsymbol{\sigma}} \cdot \boldsymbol{b})=\boldsymbol{a} \cdot \boldsymbol{b}+\imath \hat{\boldsymbol{\sigma}} \cdot(\boldsymbol{a} \times \boldsymbol{b})$, a qual convenientemente simplifica a Eq. (2.6) para sua forma mais conhecida

$$
\hat{H}=\frac{1}{2 m_{e}}\left(\hat{\boldsymbol{p}}-\frac{e}{c} \hat{\boldsymbol{A}}\right)^{2}+e \hat{\varphi}-\left(\frac{e \hbar}{2 m_{e} c}\right) \hat{\boldsymbol{\sigma}} \cdot \hat{\boldsymbol{B}} .
$$

A constante física $\mu_{B}=\left(e \hbar / 2 m_{e} c\right)=9.27410^{-21} \mathrm{ergs} / \mathrm{G}$ é o magneton de Bohr. Experimentalmente, em termos dos operadores de spin, verifica-se que última parcela da Eq. (2.8) toma a forma $-g\left(\mu_{B} / \hbar\right) \hat{\boldsymbol{S}} \cdot \hat{\boldsymbol{B}}$. A constante $g \approx 2.0023192$ é o fator giromagnético de Landé e difere de dois devido a correções puramente relativísticas. A descrição de Pauli para um único elétron num campo magnético encontrou rápida aceitação científica pois explicava o efeito Zeeman anômalo com boa precisão. Por anomalia entende-se o fator giromagnético $g \approx 2$ ao invés de $g=1$, oriundo da contribuição do momento angular orbital.

O spin é uma grandeza física inerente de partículas fundamentais como elétrons, nêutrons, prótons, mésons, quarks, glúons, fótons, etc. Existem duas grandes subdivisões na classificação de partículas quanto ao seu spin. Partículas de spin

\footnotetext{
${ }^{5}$ A discussão apropriada da versão relativística da teoria quântica requer a introdução da equação de Dirac. No que segue, e ao longo deste estudo, a versão não-relativística é suficiente para descrever os aspectos mais relevantes dos problemas e soluções apresentadas.
} 
semi-inteiro como o elétron, o próton e o nêutron são denominadas de férmions, enquanto aquelas com spin inteiro como os fótons e mésons são bósons. Um núcleo atômico pode se comportar como um férmion, caso o número de núcleons seja ímpar, ou como um bóson, caso contrário. Tanto férmions quanto bósons satisfazem ao Teorema Spin-Estatística de Pauli [48]. O teorema versa sobre as propriedades que teorias de campos relativísticas devem satisfazer para preservar a invariância de Lorentz. Salvo casos patológicos, ${ }^{6}$ conclui-se que bósons seguem a estatística de Bose-Einstein e, em contrapartida, férmions obedecem à estatística de Fermi-Dirac. As duas distribuições decorrem da indistinguibilidade das partículas fundamentais e diferem essencialmente pelo fato que a estatística Fermi-Dirac satisfaz ao princípio de exclusão de Pauli [49]. Este princípio físico estabelece que dois férmions não podem possuir simultaneamente todos os mesmos números quânticos. Geralmente, as diferenças físico-químicas entre as duas variedades de partículas são atribuídas de maneira mais impactante a baixas temperaturas, quando os fenômenos dominantes possuem natureza quântica. ${ }^{7}$ Entretanto, os efeitos do princípio da exclusão vão muito além do regime de baixas temperaturas, sendo vital para descrição eletrônica de átomos e moléculas além de estabelecer a estabilidade da matéria [50].

O princípio de exclusão possui profundas implicações matemáticas no tratamento de várias partículas. Para examinar essa questão, convém introduzir os operadores de criação $\hat{\Psi}_{\varepsilon}^{\dagger}(\vec{r})$ e destruição $\hat{\Psi}_{\varepsilon}(\vec{r})$ de partícula com spin $\varepsilon$, na posição $\vec{r}$, em relação a uma origem O. $^{8}$ Além disso, seja $|0\rangle$ o vácuo do sistema, caracterizado pela ausência de qualquer partícula. Obtém-se o estado de uma partícula, localizada na posição $\vec{r}$, por meio de $\hat{\Psi}_{\varepsilon}^{\dagger}(\vec{r})|0\rangle$. Seguindo o raciocínio anterior, o estado de duas partículas, uma na posição $\vec{r}_{1}$ e outra na posição $\vec{r}_{2}$, ambas com mesmo spin, pode ser escrito como $\hat{\Psi}_{\varepsilon}^{\dagger}\left(\vec{r}_{2}\right) \hat{\Psi}_{\varepsilon}^{\dagger}\left(\vec{r}_{1}\right)|0\rangle$ ou $\hat{\Psi}_{\varepsilon}^{\dagger}\left(\vec{r}_{1}\right) \hat{\Psi}_{\varepsilon}^{\dagger}\left(\vec{r}_{2}\right)|0\rangle$. Resta, então, certa indefinição quanto à univocidade do estado físico. Assumindo a indistinguibilidade

\footnotetext{
${ }^{6}$ Existem vários "sabores" de teoremas spin-estatística, que diferem entre si pelas restrições impostas aos campos físicos. O teorema posto adiante por Pauli apresenta grande aceitação pela comunidade científica por assumir apenas invariância de Lorentz e interações locais. Porém, vínculos espaciais, de massa e interações não-locais podem criar obstáculos conceituais. Por exemplo, bósons que interagem como partículas duras em meios unidimensionais comportam-se de modo análogo aos férmions, refletindo-se inclusive no seu espectro de energia.

${ }^{7}$ Por exemplo, o átomo He possui dois isótopos, $\mathrm{He}^{4}$ e $\mathrm{He}^{3}$, os quais possuem natureza bosônica e fermiônica respectivamente. Ambos atravessam transições de fase superfluida à baixas temperaturas $T_{\mathrm{He}^{4}}=2.17 \mathrm{~K}$ e $T_{\mathrm{He}^{3}}=2.5$ mK. A manifestação das distintas naturezas se dá pela constatação de que $T_{\mathrm{He}^{4}} \gg T_{\mathrm{He}^{3}}$, já que o He ${ }^{3}$ requer cooperação entre número par de átomos, enquanto o caso bosônico é essencialmente explicado por condensação de Bose-Einstein [20].

${ }^{8}$ Essa abordagem é mais conhecida como segunda quantização [41]. Ao contrário da descrição tradicional de uma partícula, a segunda quantização ocorre no espaço de Fock. Mais ainda, o vetor posição não é um operador, tratando-se somente de um parâmetro.
} 
das partículas, os geradores de estados de duas partículas são combinações lineares simétricas ou anti-simétricas:

$$
\begin{array}{ll}
\hat{\Psi}_{\varepsilon}^{\dagger}\left(\vec{r}_{2}\right) \hat{\Psi}_{\varepsilon}^{\dagger}\left(\vec{r}_{1}\right)+\hat{\Psi}_{\varepsilon}^{\dagger}\left(\vec{r}_{1}\right) \hat{\Psi}_{\varepsilon}^{\dagger}\left(\vec{r}_{2}\right) & \text { (simétrica), } \\
\hat{\Psi}_{\varepsilon}^{\dagger}\left(\vec{r}_{2}\right) \hat{\Psi}_{\varepsilon}^{\dagger}\left(\vec{r}_{1}\right)-\hat{\Psi}_{\varepsilon}^{\dagger}\left(\vec{r}_{1}\right) \hat{\Psi}_{\varepsilon}^{\dagger}\left(\vec{r}_{2}\right) & \text { (anti-simétrica). }
\end{array}
$$

Quando $\vec{r}_{1}=\vec{r}_{2}$, a solução anti-simétrica proíbe a existência do estado duas partículas, na mesma posição e com mesmo spin, consistente com o princípio de exclusão de Pauli. Tratam-se, portanto, de férmions: os operadores de criação fermiônicos ganham uma fase de $\pi$ mediante permutação das posições, $\hat{\Psi}_{\varepsilon}^{\dagger}\left(\vec{r}_{2}\right) \hat{\Psi}_{\varepsilon}^{\dagger}\left(\vec{r}_{1}\right)=$ $-\hat{\Psi}_{\varepsilon}^{\dagger}\left(\vec{r}_{1}\right) \hat{\Psi}_{\varepsilon}^{\dagger}\left(\vec{r}_{2}\right)$. Em geral, conclui-se que operadores fermiônicos sempre satisfazem $\hat{\Psi}_{\varepsilon}^{\dagger}(\vec{r}) \hat{\Psi}_{\varepsilon}^{\dagger}(\vec{r})=0$ bem como $\hat{\Psi}_{\varepsilon}(\vec{r}) \hat{\Psi}_{\varepsilon}(\vec{r})=0$. No caso bosônico não há introdução de fase adicional ao se permutar as posições das partículas. O estudo dos demais produtos de operadores locais de criação e destruição de partículas, em conjunto com as regras acima, permite sintetizar as relações algébricas para bósons (comutadores) e férmions (anti-comutadores):

$$
\begin{aligned}
& {\left[\hat{\Psi}_{\varepsilon^{\prime}}\left(\vec{r}^{\prime}\right), \hat{\Psi}_{\varepsilon}(\vec{r})\right]_{ \pm}=0,} \\
& {\left[\hat{\Psi}_{\varepsilon^{\prime}}^{\dagger}\left(\vec{r}^{\prime}\right), \hat{\Psi}_{\varepsilon}^{\dagger}(\vec{r})\right]_{ \pm}=0,} \\
& {\left[\hat{\Psi}_{\varepsilon^{\prime}}\left(\vec{r}^{\prime}\right), \hat{\Psi}_{\varepsilon}^{\dagger}(\vec{r})\right]_{ \pm}=\delta\left(\vec{r}^{\prime}-\vec{r}\right) \delta_{\varepsilon^{\prime} \varepsilon},}
\end{aligned}
$$

onde $[\hat{A}, \hat{B}]_{ \pm}=\hat{A} \hat{B} \pm \hat{B} \hat{A}$ é o anti-comutador (comutador) entre os operadores $\hat{A}$ e $\hat{B}$. Dito de maneira mais usual, operadores bosônicos (fermiônicos) correspondentes a partículas distintas comutam (anti-comutam).

\section{$2.2 \quad$ Operadores de spin localizados}

Um caso de grande interesse físico, matemático e, como mostraremos adiante, epidemiológico dispõe sobre operadores fortemente localizados de criação e destruição de partículas de spin 1/2. A localização aqui referida é propositalmente vaga pois pode representar tanto sítios de uma rede de Bravais [34, 51-54] como, a depender do contexto, vértices de um grafo ou rede [55]. Neste último caso, redes e grafos são amplamente utilizados para organizar e modelar uma variedade de grandezas físicas, econômicas, sociais e biológicas bem como suas interações [14, 56-59]. Maiores detalhes sobre grafos e redes são abordados no Apêndice 1. Nesta seção, 
o termo sítio é sinônimo para sítio de redes de Bravais assim como para vértice de grafos. Neste contexto, cada sítio é univocamente identificado por um número inteiro como ilustra a Figura 2.1.

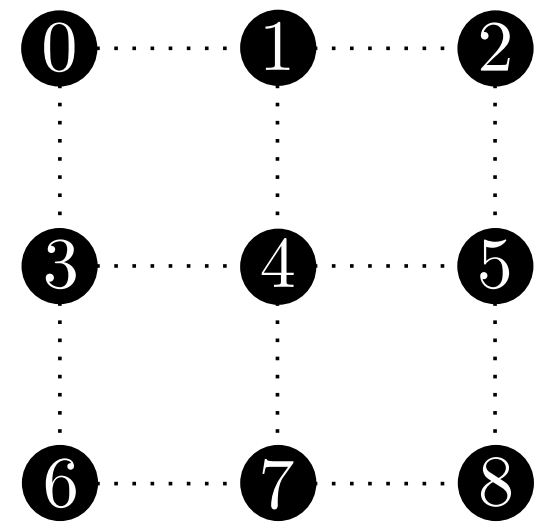

a)

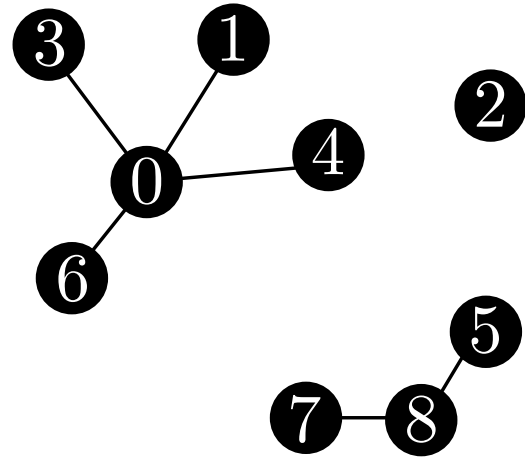

b)

Figura 2.1: Enumeração de sítios ou vértices. a) Rede regular quadrada bidimensional de com $N_{x}$ sítios na direção $\hat{x}$ e $N_{y}$ sítios na direção $\hat{y}$. Os índices $k=0,1, \ldots, N_{x} N_{y}-1$ são calculados por meio da fórmula $k=n_{x}+n_{y} N_{x}$, onde $0 \leq n_{x}<N_{x}\left(0 \leq n_{y}<N_{y}\right)$ e são os números inteiros correspondentes à coluna e à linha do sítio. b) Grafo com $N$ vértices. A ausência de regularidade nas conexões de grafos não-regulares impede a padronização da enumeração dos vértices.

O emprego de partículas de spin 1/2 ocorre por ocasião de descrever também sistemas de dois níveis. Exemplos são encontrados desde estados de infeção de agentes (susceptível ou infectado), níveis atômicos (fundamental ou excitado), materiais magnéticos $\left(m_{z}= \pm 1 / 2\right)$, etc. A base natural de $N$ sítios é obtida mediante produto direto entre as configurações de cada sítio, a saber, $|\uparrow\rangle$ e $|\downarrow\rangle$. Portanto, a base de $N$ sítios possui $2^{N}$ configurações possíveis: $\{|\downarrow \downarrow \cdots \downarrow\rangle,|\uparrow \downarrow \cdots \downarrow\rangle, \ldots,|\uparrow \uparrow \cdots \uparrow\rangle\}$. O emprego de setas para cima $\left(m_{z}=1 / 2\right)$ ou para baixo $\left(m_{z}=-1 / 2\right)$ apenas explora a familiaridade com a projeção de spin $1 / 2$. No que segue, convém enumerar cada elemento da base de $N$ sítios de spin 1/2. Um modo simples e mais apropriado para eventuais cálculos numéricos é a representação binária. Nesta representação, a configuração $\left|\sigma_{0} \sigma_{1} \cdots \sigma_{N-1}\right\rangle$ de $N$ spins é transcrita como um número inteiro $\mu$ entre 0 e $2^{N}-1$, mais precisamente,

$$
\mu=\delta_{\uparrow, \sigma_{0}} 2^{0}+\delta_{\uparrow, \sigma_{1}} 2^{1}+\cdots+\delta_{\uparrow, \sigma_{N-1}} 2^{N-1}
$$

onde $\delta_{m, n}$ é o delta de Kronecker. A Tabela 2.1 contém a enumeração da base de $N=4$ spin como exemplo. Generalização para spins maiores $s$ é facilmente obtida por meio da identificação $s=(q-1) / 2$ com $q$ inteiro positivo. Neste caso, 
Tabela 2.1: Enumeração dos elementos de base para $N=4$ spins meio.

\begin{tabular}{cccc}
\hline \hline Configuração & $\mu$ & Configuração & $\mu$ \\
\hline$|\downarrow \downarrow \downarrow \downarrow\rangle$ & 0 & $|\downarrow \downarrow \downarrow \uparrow\rangle$ & 8 \\
$|\uparrow \downarrow \downarrow \downarrow\rangle$ & 1 & $|\uparrow \downarrow \downarrow \uparrow\rangle$ & 10 \\
$|\downarrow \uparrow \downarrow \downarrow\rangle$ & 2 & $|\downarrow \uparrow \downarrow \uparrow\rangle$ & 11 \\
$|\uparrow \uparrow \downarrow \downarrow\rangle$ & 3 & $|\uparrow \uparrow \downarrow \uparrow\rangle$ & 12 \\
$|\downarrow \downarrow \uparrow \downarrow\rangle$ & 4 & $|\downarrow \downarrow \uparrow \uparrow\rangle$ & 13 \\
$|\uparrow \downarrow \uparrow \downarrow\rangle$ & 5 & $|\uparrow \downarrow \uparrow \uparrow\rangle$ & 14 \\
$|\downarrow \uparrow \uparrow \downarrow\rangle$ & 6 & $|\downarrow \uparrow \uparrow \uparrow\rangle$ & 15 \\
$|\uparrow \uparrow \uparrow \downarrow\rangle$ & 7 & $|\uparrow \uparrow \uparrow \uparrow\rangle$ & \\
\hline \hline
\end{tabular}

a enumeração $\mu^{\prime}$ das configurações $\left|\tau_{0} \tau_{1} \cdots \tau_{N-1}\right\rangle\left(\tau_{k}=0,1, \ldots, q-1\right)$ utiliza a representação $q$-ária:

$$
\mu^{\prime}=\sum_{k=0}^{N-1} \tau_{k} q^{k}=\sum_{k=0}^{N-1}\left(\sum_{m=0}^{q-1} m \delta_{\tau_{k}, m}\right) q^{k} .
$$

No que tange este estudo, emprestamos o termo localização pela habilidade de identificar univocamente um dado operador $\hat{O}_{k}$ com um único número inteiro $k=0,1, \ldots, N-1$ dentro de um conjunto de $N$ números inteiros ordenados. Tais objetos são análogos aos operadores de campo de teorias quânticas de campo. No que concerne essa tese, consideramos apenas os operadores localizados de spin $\hat{S}_{\alpha, k}$ com $\alpha= \pm, x, y, z$. Esses operadores possuem grande aplicabilidade por dois motivos. Primeiro, simulam sistemas de dois níveis, permitindo a descrição de transições atômicas e moleculares [24, 25, 60-65], de sistemas ferro- e antiferromagnéticos bem como suas transições de fase $[17,34,52,54,66-71]$. Segundo, suas propriedades algébricas podem ser exploradas tanto do ponto de vista bosônico como fermiônico. Em princípio, essa afirmação causa estranheza pois conflita com o teorema SpinEstatística [48], que separa partículas em bósons ou férmions. O problema todo reside na localização dos operadores.

A descrição das propriedades algébricas de operadores fundamenta-se nos comutadores e anti-comutadores. Os operadores localizados de spin $1 / 2$ satisfazem as seguintes relações algébricas:

$$
\begin{aligned}
{\left[\hat{S}_{k z}, \hat{S}_{j \pm}\right] } & = \pm \hbar \hat{S}_{k \pm} \delta_{k, j} \\
{\left[\hat{S}_{k \pm}, \hat{S}_{j \pm}\right] } & =0 \\
{\left[\hat{S}_{k+}, \hat{S}_{j-}\right] } & =2 \hbar \hat{S}_{k z} \delta_{k, j} \\
\left\{\hat{S}_{k+}, \hat{S}_{k-}\right\} & =\hbar^{2} \mathbb{1} .
\end{aligned}
$$


As relações das Eqs. (2.16a) e (2.16c) são nada menos do que as constantes de estrutura para $k=j$. Para $k \neq j$, as relações das Eqs. (2.16b) e (2.16c) são compatíveis com aquelas de operadores bosônicos. O problema aparece quando se considera a Eq. (2.16d), que por sua vez é compatível com a formulação fermiônica. Posto de outro modo, os operadores localizados de spin-1/2 comutam em sítios distintos mas anti-comutam localmente, portanto, não possuem caráter nem bosônico nem fermiônico completo.

A indeterminação da natureza fermiônica ou bosônica produz consequências relevantes, especialmente para a contagem de estados e degenerescências, ponto particularmente sensível para Mecânica Estatística. De fato, não há descrição precisa de quasipartículas neste contexto, dificultando até mesmo a interpretação teórica do modelo. A alternativa mais comum para solucionar este problema é bastante pragmática: já que não há caráter pré-definido, escolhe-se um via bosonificação ou fermionização. No que segue, apresentamos brevemente uma revisão sobre ambos os métodos, com interpretações próprias e acumuladas ao longo da experiência com estas técnicas, que ultimamente deram origem ao tema central deste tese. Destaca-se que esses métodos, apesar do grande desenvolvimento teórico nas áreas de sistemas exatamente integráveis, não encontraram grandes aplicações em outras áreas da Física. Isso não se deve à impossibilidade de aplicação metodológica mas sim ao desconhecimento das técnicas em si, muito específicas e, portanto, limitada a uma pequena audiência científica. Por este motivo, essa revisão preocupa-se principalmente em expor as principais características e passos dos métodos de fermionização e bosonificação. Contudo, alguns detalhes técnicos são expostos, enfatizando os problemas e soluções associadas, geralmente negligenciados em publicações relevantes devido à assunção equivocada de conhecimento geral sobre o assunto.

\subsection{Fermionização}

A fermionização de operadores decorre de transformações unitárias, portanto, inversíveis e válidas para todo o espectro. Ela traz à tona o caráter fermiônico, em detrimento do bosônico, por meio da introdução de correlações não-locais conhecidas como transformações de Jordan-Wigner [10, 72, 73]. Tais correlações não-locais resultam no comportamento esperado de férmions, quando considerados os efeitos 
de localização dos operadores. Essa situação é análoga ao caso de dois elétrons separados por uma grande distância: seus operadores são certamente fermiônicos - portanto, seus spinores são antisimétricos e correlacionados - mas suas funções de onda espacial possuem superposição negligenciável, de modo que os elétrons são independentes, para todos os outros fins.

A transformação de Jordan-Wigner, ou fermionização, $\hat{U}_{k}$ transforma o operador de spin $\hat{S}_{-k}\left(\hat{S}_{k+}\right)$ no operador $\hat{c}_{k}\left(\hat{c}_{k}^{\dagger}\right)$,

$$
\begin{aligned}
\hat{c}_{k} & =\hbar^{-1} \hat{U}_{k} \hat{S}_{k-}, \\
\hat{c}_{k}^{\dagger} & =\hbar^{-1} \hat{S}_{k+} \hat{U}_{k}^{\dagger}, \\
\hat{U}_{k} & =\exp \left(\frac{\imath \pi}{\hbar^{2}} \sum_{l=0}^{k-1} \hat{S}_{l+} \hat{S}_{l-}\right) .
\end{aligned}
$$

Destaca-se que a somatória na transformação unitária da Eq. (2.17c) do sítio $k$ incrementa seu valor por $\imath \pi$ conforme o número de spins $\uparrow$ entre o sítio inicial e o sítio $k-1$. Dessas definições, decorrem duas propriedades importantes, a saber, o operador número de partículas, $\hat{n}_{k}$, permanece inalterado

$$
\hat{n}_{k} \equiv \hat{c}_{k}^{\dagger} \hat{c}_{k}=\hat{S}_{k+} \hat{S}_{k-} \hbar^{-2}
$$

e os operadores $\hat{c}_{k}$ são fermiônicos pois

$$
\begin{aligned}
& \left\{\hat{c}_{k}^{\dagger}, \hat{c}_{j}\right\}=\delta_{k j} \mathbb{1}, \\
& \left\{\hat{c}_{k}, \hat{c}_{j}\right\}=0, \\
& \left\{\hat{c}_{k}^{\dagger}, \hat{c}_{j}^{\dagger}\right\}=0 .
\end{aligned}
$$

A verificação das Eqs. (2.19a-2.19c) para $k \geqslant j$ e $j=0, \ldots, N-1$ decorre diretamente das Eqs. (2.17a-2.17c). A Figura 2.2 ilustra um diagrama para visualizar o processo de fermionização. Por fim, note-se que a transformação de Jordan-Wigner nas Eqs. (2.17a-2.17c) muda a interpretação dos spins para cima $\uparrow$ para férmions sem spin.

Antes de mostrar uma breve aplicação do método, convém discutir sobre o ponto fraco da fermionização: a invariância translacional. A fim de simplificar a discussão, consideramos o caso mais simples de uma rede unidimensional com condições periódicas de contorno. Sob tais circunstâncias, a periodicidade do contorno naturalmente implica $\hat{S}_{N \pm}=\hat{S}_{0 \pm}$. Embora essa propriedade seja trivial para os 
a)

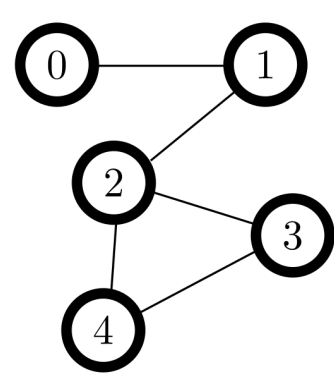

b)

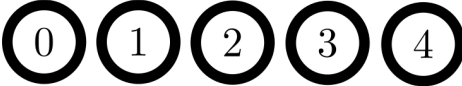

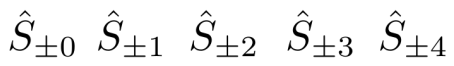

c)

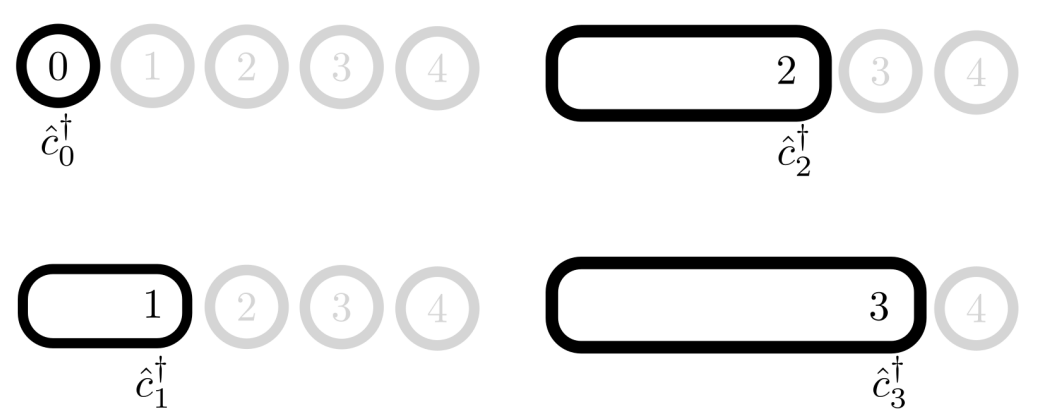

Figura 2.2: Diagrama para fermionização de operadores localizados. O sistema possui $N=5$ sítios com interconexões representadas em a). Em b), os operadores de spin $\hat{S}_{ \pm k}$ são organizados de acordo com a ordenação dos sítios correspondentes. Operadores $\hat{S}_{ \pm k}$ de sítios distintos não possuem vínculos uns com os outros, resultando na relação bosônica $\left[\hat{S}_{ \pm j}, \hat{S}_{ \pm k}\right]=0$ para $j>k$. Em c), a transformação unitária de Jordan-Wigner correlaciona os operadores $\hat{c}_{k}^{\dagger}$ de diferentes sítios, estendendo-se como uma cauda, produzindo apenas relações fermiônicas.

operadores localizados de spin, o mesmo não ocorre para os operadores fermiônicos $\hat{c}_{N}$. Por exemplo, a partir da identidade $\hat{S}_{N+}=\hat{S}_{0+}$, calcula-se

$$
\hat{c}_{N}^{\dagger} \exp \left(\imath \pi \sum_{l=0}^{N-1} \hat{c}_{l}^{\dagger} \hat{c}_{l}\right) \equiv \hat{c}_{0}^{\dagger},
$$

ou seja, a condição de contorno antes periódica torna-se periódica ou anti-periódica, a depender unicamente do número de férmions no sistema. No caso específico de cadeias unidimensionais, existe uma solução elegante para o problema como mostraremos mais adiante, cujo principal efeito é modificar os possíveis valores de momenta. É fácil ver que o incremento da dimensionalidade, por exemplo, introduz imprecisão na definição da condição de contorno, pois esta passa a depender da maneira segundo a qual os operadores foram enumerados. Essa subjetividade impede a formulação de generalizações. ${ }^{9}$

\footnotetext{
${ }^{9}$ A modificação das condições de contorno não é em si uma surpresa. Grosso modo, o contorno especifica os vetores de onda disponíveis aos modos fermiônicos. Logo, modificações no contorno estão em consonância com o ansatz de Bethe [34, 74-76], que estabelece a resolução de sistemas fortemente correlacionados via escolha adequada de vetores de onda, não necessariamente raízes da unidade.
} 


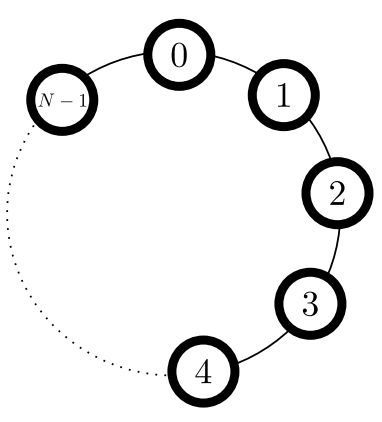

Figura 2.3: Cadeia XY de spin 1/2 com $N$ sítios e condição periódica de contorno. Sítios são unicamente identificados por um número inteiro $k=0,1, \ldots, N-1$ onde cada sítio carrega um único spin, $\left|\uparrow_{k}\right\rangle$ ou $\left|\downarrow_{k}\right\rangle$ no eixo de quantização $z$. A característica marcante da cadeia periódica é a conexão apenas entre vizinhos mais próximos, ou seja, o sítio $k$ conecta-se com os sítios $k \pm 1$, o que gera invariância por translações na variável $k$.

\subsection{Fermionização: aplicação no modelo XY}

O modelo XY unidimensional de spin 1/2 figura o exemplo mais simples e pedagógico sobre a transformação de Jordan-Wigner. Nesta cadeia unidimensional de spins, $N$ sítios são dispostos sobre um círculo de tal modo que cada sítio conectase apenas com seus vizinhos mais próximos, como mostra a Figura (2.3). O operador Hamiltoniano do sistema é

$$
\hat{H}_{X Y}=-\frac{g}{\hbar^{2}} \sum_{k=0}^{N-1}\left(\hat{S}_{k+} \hat{S}_{k+1-}+\hat{S}_{k-} \hat{S}_{k+1+}\right),
$$

com constante de acoplamento $g \approx 1-3 \mathrm{meV}$, e descreve o movimento cinético de spin. Aqui, consideramos a condição twisted de contorno: os operadores de spin localizados ganham uma fase topológica $\phi \equiv \phi(J)$ a cada circuitação pela rede, isto é

$$
\hat{S}_{N \pm}=\mathrm{e}^{ \pm \imath \phi} \hat{S}_{0 \pm}
$$

A condição periódica é recuperada quando $\phi=0$.

O Hamiltoniano da Eq. (2.21) conserva explicitamente o número total de spin (magnetização), fato este que decorre da simetria $U(1)$. Portanto, exige-se que a fermionização também preserve o número de férmions sem spin. Substituindo-se as Eqs. (2.17a-2.17c) na Eq. (2.21), obtém-se

$$
\begin{aligned}
\hat{n} & \equiv \sum_{k} \hat{c}_{k}^{\dagger} \hat{c}_{k} \\
\hat{H}_{X Y} & =-g \sum_{k=0}^{N-2}\left(\hat{c}_{k}^{\dagger} \hat{c}_{k+1}+\hat{c}_{k+1}^{\dagger} \hat{c}_{k}\right)+
\end{aligned}
$$




$$
-g\left[\mathrm{e}^{-\imath \pi(\hat{n}+1)+\imath \phi} \hat{c}_{N-1}^{\dagger} \hat{c}_{0}+\hat{c}_{0}^{\dagger} \hat{c}_{N-1} \mathrm{e}^{\imath \pi(\hat{n}+1)-\imath \phi}\right] .
$$

A contribuição advinda do contorno na Eq. (2.21) carrega informação a respeito de todo o sistema por meio do operador número total de férmions, $\hat{n}$. Todavia, devido à conservação do número total de férmions, $\left[\hat{n}, \hat{H}_{X Y}\right]=0$, existe uma transformação de gauge de primeiro tipo ${ }^{10}$ que espalha igualmente a fase do contorno por todos os operadores fermiônicos. A transformação de gauge consiste em:

$$
\begin{aligned}
\omega_{n} & \equiv \frac{\imath \pi(n+1)-\phi}{N}, \\
\hat{\psi}_{k}^{\dagger} & \equiv \mathrm{e}^{-\imath \omega_{n} k} \hat{c}_{k}^{\dagger}, \\
\hat{\psi}_{k} & \equiv \mathrm{e}^{+\imath \omega_{n} k} \hat{c}_{k},
\end{aligned}
$$

restrita ao setor com $n$ férmions. Aplicando-se as transformações das Eqs. (2.25b) e (2.25c) na Eq. (2.24), temos

$$
\hat{H}_{X Y}=-g \sum_{k=0}^{N-1}\left(\mathrm{e}^{-\imath \omega_{n}} \hat{\psi}_{k}^{\dagger} \hat{\psi}_{k+1}+\mathrm{e}^{+\imath \omega_{n}} \hat{\psi}_{k+1}^{\dagger} \hat{\psi}_{k}\right),
$$

cuja solução é trivialmente obtida mediante uso dos operadores de criação e destruição de férmions no espaço de momenta ${ }^{11} q \in\{0,2 \pi / N, \ldots, 2 \pi(N-1) / N\}$ :

$$
\begin{aligned}
& \hat{\Psi}_{q}=\frac{1}{\sqrt{N}} \sum_{k=0}^{N-1} \mathrm{e}^{-\imath q k} \hat{\psi}_{k} \\
& \hat{\Psi}_{q}^{\dagger}=\frac{1}{\sqrt{N}} \sum_{k=0}^{N-1} \mathrm{e}^{\imath q k} \hat{\psi}_{k}^{\dagger} .
\end{aligned}
$$

Em termos dos operadores $\hat{\Psi}_{q_{m}}$ e $\hat{\Psi}_{q_{m}}^{\dagger}$,

$$
\hat{H}_{X Y}=-2 g \sum_{j=0}^{N-1} \cos \left(q_{j}-\omega_{n}\right) \hat{\Psi}_{q_{j}}^{\dagger} \hat{\Psi}_{q_{j}},
$$

com momento $q_{j}=2 \pi j / N$. Naturalmente, quando $N \rightarrow \infty$, a fase oriunda da condição de contorno torna-se irrelevante de acordo com a Eq. (2.25a). Todavia,

\footnotetext{
${ }^{10}$ Coincidentemente, a conservação de carga elétrica também é obtida por meio da invariância de gauge de primeiro tipo em teorias de campos [41, 77]. Destaca-se a semelhança com a conservação da carga elétrica, cuja base fundamenta-se na conservação simultânea de partículas e anti-partículas.

${ }^{11} \mathrm{O}$ emprego da transformada de Fourier baseia-se na invariância por permutações cíclicas $\hat{P}$, isto é, os operadores permanecem invariantes após $N$ permutações cíclicas, $\hat{P}^{N}=\mathbb{1}$, produzindo os números de onda $q=2 \pi m / N$, com $m=0,1, \ldots, N-1$. A permutação finita é então associada via teorema de Cayley às translações espaciais. Neste caso, entretanto, também é possível interpretar como rotações espaciais em relação a uma origem situada no eixo da cadeia.
} 
a paridade nunca é desprezável pois a razão $n / N$ é sempre finita entre zero e a unidade, mesmo quando expressa na zona de Brillouin correspondente [51].

A menor energia é facilmente calculada da seguinte maneira. Seja $q_{0}^{\prime}$ o primeiro valor de momento com contribuição negativa para a energia total, isto é, $\cos \left(q_{0}^{\prime}\right) \geqslant 0$ ou, para a primeira zona de Brillouin, $q_{0}^{\prime}=-\pi / 2+(\pi-\phi) / N$. O caso de banda semi-preenchida $n=N / 2$ com momento nulo $Q=0$ define o estado fundamental quando $\phi=0$. Como a condição de contorno twisted apenas desloca a fase da função periódica na Eq. (2.28), o número de partículas do estado fundamental não se altera. Logo, a energia $E_{0}$ do estado fundamental é expressa como

$$
\frac{E_{0}}{-2 g}=\sum_{m=0}^{N / 2-1} \cos \left(q_{0}^{\prime}+\frac{2 \pi m}{N}\right)=\frac{\cos \phi / N}{\sin \pi / N} .
$$

No limite assintótico $N \rightarrow \infty$, a expressão toma a forma mais familiar

$$
E_{0}=-\frac{2 g L}{\pi}-\frac{\pi v_{s} c(\phi)}{6 N}+O\left(N^{-3}\right)
$$

onde $v_{s}=2 g$ é a velocidade de propagação da excitação do espectro fermiônico, e $c(\phi)=1-3(\phi / \pi)^{2}$ é a anomalia conforme ou carga conforme da teoria de campo correspondente [32, 34-36, 78].

A anomalia conforme caracteriza a teoria, similar aos pontos críticos de uma transição crítica de fase $[16,33,54,79]$. Porém, o papel de $c$ é bem mais amplo: o conhecimento de seu valor é suficiente para identificar univocamente a teoria de campo correspondente. ${ }^{12}$ Essa propriedade contrasta com aquela proveniente do grupo de Renormalização de Wilson [54, 79, 82], que requer todos os pontos críticos e expoentes críticos correspondentes para suprir a mesma tarefa. Essa propriedade extremamente poderosa decorre da teoria conforme bidimensional ${ }^{13}$ proposta por Zamolodchikov, Belavin e Polyakov nos anos 80 [83, 84], cujo grupo de simetria abrange todas as funções analíticas em $\mathbb{C}$, por meio da álgebra de Virasoro [32]. ${ }^{14}$ Um resultado notável da Eq. (2.30) faz-se presente na dependência da carga conforme com a fase $\phi$, que remete à teoria de transmutação topológica de modelos: com

\footnotetext{
${ }^{12} \mathrm{O}$ exemplo mais famoso é o modelo de Ising bidimensional cuja anomalia conforme vale $c=1 / 2$. O modelo de gás de Coulomb possui $c=1$, mesmo caso do modelo de spin- $1 / 2 \mathrm{XY}$ e a região crítica do modelo de spin-1/2 XXZ $[68,76,80,81]$.

${ }^{13} \mathrm{~A}$ conexão entre modelos quânticos unidimensionais e teorias de campos em $1+1$ dimensões ocorre mediante tanto da matriz de transferência [53] como também pela formulação de integrais de trajetória de Feynmann [41].

${ }^{14}$ Os geradores $L_{m}$ da álgebra de Virasoro satisfazem $\left[L_{m}, L_{n}\right]=(m-n) L_{m+n}+(c / 12)\left(n^{3}-n\right) \delta_{n,-m}$, onde $c$ é a anomalia conforme. Dentre os geradores $L_{m}$, encontram-se todas as potências $z^{m}$ bem como suas derivadas $\partial^{m}$ no plano complexo.
} 
condição periódica $\phi$ apropriada, poder-se-ia investigar um dado modelo utilizando um sistema bastante distinto. Esse é o caso, por exemplo, do modelo XY e o modelo de Ising quando $\phi=\pi / \sqrt{6}$.

Existem duas leis de conservação independentes na Eq. (2.28), a saber, a conservação do número de férmions $n$ e a a conservação do momento $Q$, dois excelentes números quânticos. Logo, também existem dois tipos distintos de transições fermiônicas como a Figura 2.4 ilustra. A primeira transição assinala incremento de energia devido à criação ou destruição de $\delta n$ férmion, $n \rightarrow n \pm \delta n$, e corresponde ao potencial químico. A segunda transição ocorre devido à aborção ou emissão de um quantum de excitação do sistema - uma emissão de uma onda de spin, por exemplo -, que causa a mudança $\delta Q$ do setor de momento, $Q \rightarrow Q \pm \delta Q$. As energias provenientes da Eq. (2.28) não possuem gaps no limite assintótico $N \rightarrow \infty$. As excitações mínimas de energia tomam a forma $E(\delta Q)-E_{0} \approx \delta Q v_{s}$, em clara analogia com a relação relativística de energia para partículas sem massa. Em fechamento, a ferminiozação provê acima de tudo, um contexto intuitivo e simples para se investigar excitações do sistema. 


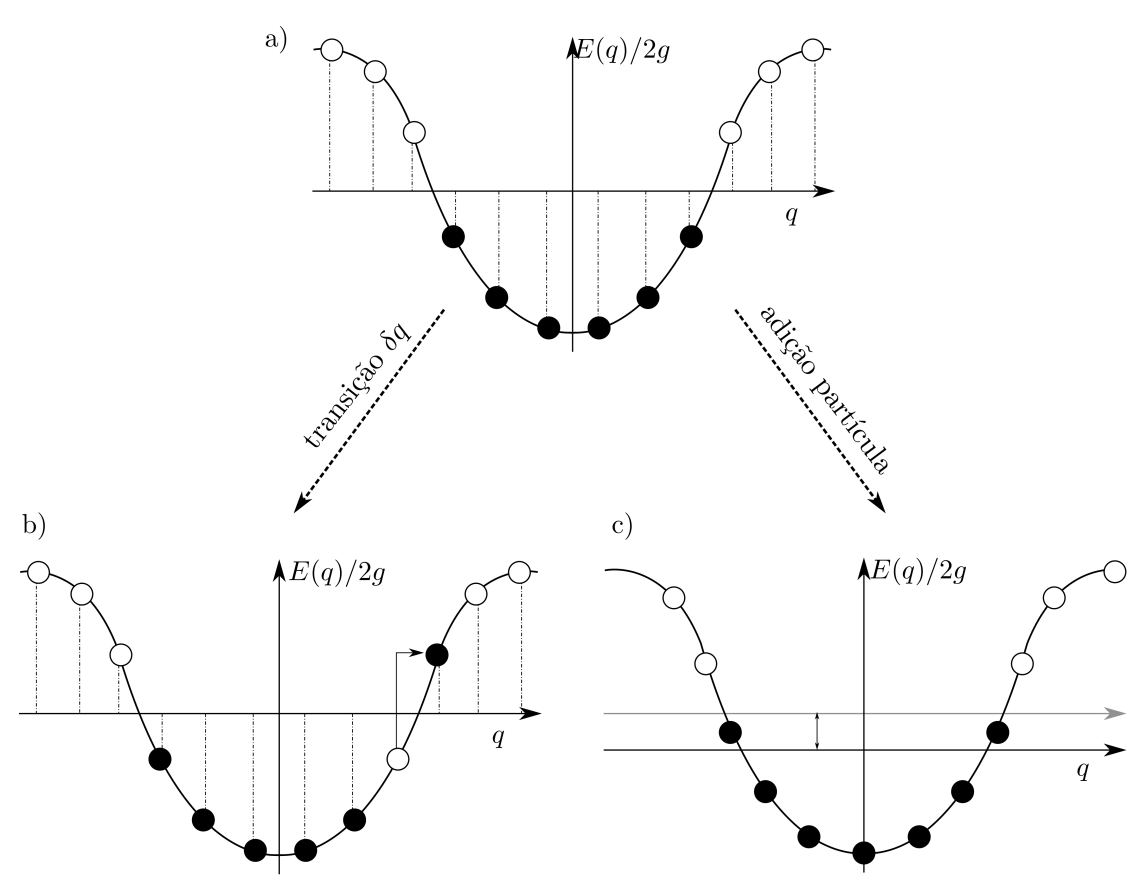

Figura 2.4: Níveis de energia e transições da cadeia quântica XY na interpretação fermiônica com $N=12$ sítios. Os níveis ocupados (não-ocupados) são representados por círculos preenchidos (vazios). a) Configuração do estado de menor energia $E_{0}$, com $n=N / 2$ níveis de energia negativa, com momento total $Q=0$. b) Um férmion é excitado para o próximo nível de energia mediante injeção de momento $\delta q=2 \pi / N$. Apesar da transição, os níveis de energia dentro de uma mesma zona de Brillouin permanecem inalterados. c) A adição de um novo férmion modifica a distribuição dos números $q$ permitidos de tal maneira que $Q$ permanece inalterado, porém modifica todos os níveis de energia. Essa modificação resulta num acréscimo de energia em relação ao nível fundamental $E_{0}$.

\subsection{Bosonificação}

Apesar do nome curioso, o processo de bosonificação apenas exprime o caráter bosônico de operadores localizados de momento angular. Ao contrário da fermionização, a bosonificação não é formulada a partir de uma lei de conservação do número partículas, pois pode tratar tanto de partículas bosônicas (que podem ser conservadas) quanto excitações bosônicas. Neste último caso, não faz sentido impor lei de conservação já que o potencial químico é nulo. Assim como nas seções anteriores sobre fermionização, os detalhes mais técnicos serão abordados nos capítulos apropriados como parte de trabalhos originais. Nesta seção, levantamos os aspectos mais gerais e a problemática decorrente da bosonificação.

A remoção do caráter fermiônico dos operadores de spin, expresso pela Eq. (2.16d), requer diversos passos para sua estruturação lógica. A pedra fundamental, claro, consiste nos operadores de criação e destruição de modos normais de 
$N$ osciladores harmônicos distinguíveis, $\hat{a}_{k}^{\dagger}$ e $\hat{a}_{k}$, respectivamente. Suas propriedades algébricas são

$$
\begin{aligned}
& {\left[\hat{a}_{k}, \hat{a}_{j}^{\dagger}\right]=\mathbb{1} \delta_{k j},} \\
& {\left[\hat{a}_{k}^{\dagger}, \hat{a}_{j}^{\dagger}\right]=0,} \\
& {\left[\hat{a}_{k}, \hat{a}_{j}\right]=0 .}
\end{aligned}
$$

De certo modo, estes operadores são aqueles que realmente caracterizam e definem modos bosônicos. Logo, nada mais justo que os incluir no processo de bosonificação, ou mais precisamente descrever operadores de momento angular em termos de operadores do oscilador harmônico [11]. De fato, essa ideia fundamenta a formulação da teoria quântica do momento angular por Schwinger [19].

A principal correspondência entre as duas classes de operadores decorre dos quanta de momento angular e de energia:

$$
\begin{aligned}
\left|s_{k},-s\right\rangle & \rightarrow\left|0_{k}\right\rangle_{\text {oscilador }}, \\
\left|s_{k},-s+1\right\rangle & \rightarrow\left|1_{k}\right\rangle_{\text {oscilador }}, \\
\vdots & \vdots \\
\left|s_{k},+s\right\rangle & \rightarrow\left|2 s_{k}\right\rangle_{\text {oscilador }} .
\end{aligned}
$$

Ora, isso sugere a seguinte representação de $\hat{S}_{k z}$ em termos de operadores do $k$-ésimo oscilador harmônico,

$$
\hat{S}_{k z} \equiv \hbar\left(\hat{a}_{k}^{\dagger} \hat{a}_{k}-s_{k}\right)
$$

com $s_{k}=s=1 / 2$ posto o tratamento de operadores de spin 1/2. Mais ainda, tal correspondência implica que os operadores $\hat{S}_{k+}\left(\hat{S}_{k-}\right)$ apresentam dependência linear com os operadores $\hat{a}_{k}^{\dagger}\left(\hat{a}_{k}\right)$, ou seja, $\hat{S}_{k+}=\hbar \hat{B}_{k}^{\dagger} \hat{a}_{k}^{\dagger}$ e $\hat{S}_{k-}=\hbar \hat{a}_{k} \hat{B}_{k}$. Por hipótese, o operador $\hat{B}_{k} \equiv \hat{B}_{k}\left(\hat{a}_{k}^{\dagger} \hat{a}_{k}\right)$ pode ser, no máximo, uma função analítica do operador número $\hat{a}_{k}^{\dagger} \hat{a}_{k}$. Logo,

$$
\left[\hat{S}_{k z}, \hat{S}_{k+}\right]=\hbar \hat{B}_{k}^{\dagger}\left[\hat{S}_{k z}, \hat{a}_{k}^{\dagger}\right]=\hbar \hat{S}_{k+}
$$

Expressão análoga segue para $\hat{S}_{k-}$. Por fim, o comutador $\left[\hat{S}_{k+}, \hat{S}_{k-}\right]=2 \hbar \hat{S}_{k z}$ determina a forma operatorial de $\hat{B}_{k}$, produzindo as transformações locais de Holstein- 
Primakoff:

$$
\begin{aligned}
& \hat{S}_{k+} \equiv \hbar\left(\mathrm{e}^{-\imath \hat{\Phi}_{k}} \sqrt{2 s_{k}+1-\hat{a}_{k}^{\dagger} \hat{a}_{k}}\right) \hat{a}_{k}^{\dagger}, \\
& \hat{S}_{k-} \equiv \hbar \hat{a}_{k}\left(\mathrm{e}^{+\imath \hat{\Phi}_{k}} \sqrt{2 s_{k}+1-\hat{a}_{k}^{\dagger} \hat{a}_{k}}\right),
\end{aligned}
$$

onde os operadores locais de fase $\Phi_{k} \equiv \Phi_{k}\left(\hat{a}_{k}^{\dagger} \hat{a}_{k}\right)$ são funções analíticas arbitrárias do operador número $\hat{a}_{k}^{\dagger} \hat{a}_{k}$ para cada sítio $k{ }^{15}$ Destaca-se o papel da raiz quadrada: ela delimita o crescimento dos estados do oscilador harmônico, reproduzindo o padrão dos operadores de levantamento do momento angular,

$$
\left(\mathrm{e}^{-\imath \Phi_{k}} \sqrt{2 s_{k}+1-\hat{a}_{k}^{\dagger} \hat{a}_{k}} \hat{a}_{k}^{\dagger}\right)\left|2 s_{k}\right\rangle=0
$$

Outra maneira de se obter as transformações Eqs. (2.34a) e (2.34b) parte da seguinte igualdade:

$$
\begin{aligned}
\hat{S}_{k+}\left|s_{k}, m_{k}\right\rangle & =\hbar \sqrt{s_{k}\left(s_{k}+1\right)-m_{k}\left(m_{k}+1\right)}\left|s_{k}, m_{k}+1\right\rangle \\
& =\hbar \sqrt{s_{k}\left(s_{k}+1\right)-\left(\hat{S}_{k z} / \hbar-1\right)\left(\hat{S}_{k z} / \hbar\right)}\left|s_{k}, m_{k}+1\right\rangle \\
& =\hbar \sqrt{s_{k}\left(s_{k}+1\right)-\left(s_{k}-\hat{a}_{k}^{\dagger} \hat{a}_{k}+1\right)\left(s_{k}-\hat{a}_{k}^{\dagger} \hat{a}_{k}\right)}\left|n_{k}+1\right\rangle_{\text {oscilador }} \\
& =\hbar \sqrt{2 s_{k}+1-\hat{a}_{k}^{\dagger} \hat{a}_{k}} \sqrt{\hat{a}_{k}^{\dagger} \hat{a}_{k}}\left[\left(\hat{a}_{k}^{\dagger} \hat{a}_{k}\right)^{-1 / 2} \hat{a}_{k}^{\dagger}\left|n_{k}\right\rangle_{\text {oscilador }}\right] \\
\therefore \hat{S}_{k+}\left|s_{k}, m_{k}\right\rangle & =\hbar \sqrt{2 s_{k}+1-\hat{a}_{k}^{\dagger} \hat{a}_{k}} \hat{a}_{k}^{\dagger}\left|n_{k}\right\rangle_{\text {oscilador }} .
\end{aligned}
$$

Emprega-se o mesmo raciocínio para o operador $\hat{S}_{k-}$, cujo resultado é a Eq. (2.34b).

O mesmo princípio das Eqs. (2.34a) e (2.34b) também serve como guia para a bosonificação do momento angular total. Neste caso, não se busca a expressão de bósons como partículas localizadas. Ao contrário, deseja-se exprimir os modos normais ou excitações bosônicas do sistema de $N$ spins $1 / 2$, em termos dos operadores bosônicos $\hat{\mathcal{A}}$ e $\hat{\mathcal{A}}^{\dagger}$. Tais excitações recebem o nome de ondas de spin. Sejam os operadores globais de momento angular

$$
\hat{S}_{z}=\sum_{k=0}^{N-1} \hat{S}_{k z}
$$

${ }^{15}$ Convém salientar que, como ocorre na reta final de toda redação científica, a consideração de operadores de fase topológicos $\hat{\Phi}_{k}\left(\hat{n}_{k}\right)$ se deu durante a escrita da tese. Esses operadores são consistentes com as relações de comutação dos geradores das rotações, condição suficiente para a descrição física de $\hat{S}_{k \pm, z}$. Por outro lado, evidencia o homomorfismo entre operadores de momento angular e de osciladores. Enquanto esse problema é mera questão burocrática para um único sítio, o mesmo não é verdade para $N>1$, já que pode produzir diferenças de fases relativas. A discussão acerca desse tópico e seus efeitos não possui ampla divulgação na literatura especializada e, merece estudo a parte. 


$$
\begin{aligned}
& \hat{S}_{+}=\sum_{k=0}^{N-1} \hat{S}_{k+}, \\
& \hat{S}_{-}=\sum_{k=0}^{N-1} \hat{S}_{k-}, \\
& \hat{S}^{2}=\hat{S}_{z}^{2}+\frac{1}{2}\left\{\hat{S}_{+}, \hat{S}_{-}\right\} .
\end{aligned}
$$

O operador de quadrático de Casimir $\hat{S}^{2}$ possui autovalores $\hbar^{2} S(S+1) \operatorname{com} S=$ $N s, N s-1, N s-2, \ldots$ inteiro ou semi-inteiro a depender da paridade de $N$. Logo, de acordo com a teoria do momento angular, os $2 S+1$ autovalores de $\hat{S}_{z}$ são $m=$ $-S,-S+1, \ldots,+S$, para cada setor $S$. Portanto, as transformações globais de Holstein-Primakoff são dadas por

$$
\begin{aligned}
& \hat{S}_{+} \equiv \hbar\left(\mathrm{e}^{-i \hat{\Phi}} \sqrt{2 S+1-\hat{\mathcal{A}}^{\dagger} \hat{\mathcal{A}}}\right) \hat{\mathcal{A}}^{\dagger} \\
& \hat{S}_{-} \equiv \hbar \hat{\mathcal{A}}\left(\mathrm{e}^{+\imath \hat{\Phi}} \sqrt{2 S+1-\hat{\mathcal{A}}^{\dagger} \hat{\mathcal{A}}}\right)
\end{aligned}
$$

Assim como no caso local, o operador $\hat{\Phi} \equiv \hat{\Phi}\left(\hat{\mathcal{A}}^{\dagger} \hat{\mathcal{A}}\right)$ é uma função exclusiva do operador número. Por ser uma fase global, seu efeito pode ser omitido para sistemas homogêneos e isotrópicos. ${ }^{16}$

A grande diferença entre as transformações de Holstein-Primakoff locais expressas Eqs. (2.34a) e (2.34b) de suas versões globais nas Eqs. (2.38a) e (2.38b) decorre dos distintos valores de spin dos sítios $s$ e do sistema $S$. Para $N \gg 1$ segue que $S=N s \gg 1$ mesmo que $s \sim O(1)$, devido à adição do momento angular total. Essa observação, tanto quanto óbvia, estabelece que os diferentes regimes físicos podem ser caracterizados por uma densidade

$$
\zeta=\frac{\left\langle\hat{\mathcal{A}}^{\dagger} \hat{\mathcal{A}}\right\rangle}{2 S+1}
$$

Caso o sistema de $N$ spins apresente homogeneidade, então é fácil mostrar que $\zeta$ é uma constante e único parâmetro necessário para descrever qualquer propriedade de transporte ou susceptibilidade correspondente ao sistema.

Adicionalmente, exitem dois regimes de grande interesse físico cuja investigação é bastante simplificada por meio da bosonificação de modos globais. No primeiro caso, existem poucas excitações globais em setores de $S$ elevado, isto é, a densidade

\footnotetext{
${ }^{16}$ Um estudo sobre as consequências da omissão da fase global $\hat{\Phi}$ para sistemas não homogêneos ou isotrópicos encontra-se em andamento, bem como seu relacionamento com as fases locais $\hat{\Phi}_{k}$.
} 
média de ondas de spin satisfaz a condição $\zeta \ll 1$. Nesta situação particular, as Eqs. (2.38a) e (2.38b) adquirem as seguintes expansões em séries de potência:

$$
\begin{aligned}
& \hat{S}_{+}=\hbar \sqrt{2 S+1}\left[\hat{\mathcal{A}}^{\dagger}-\frac{1}{2}\left(\frac{\hat{\mathcal{A}}^{\dagger} \hat{\mathcal{A}}}{2 S+1}\right) \hat{\mathcal{A}}^{\dagger}+\cdots\right], \\
& \hat{S}_{-}=\hbar \sqrt{2 S+1}\left[\hat{\mathcal{A}}-\frac{\hat{\mathcal{A}}}{2}\left(\frac{\hat{\mathcal{A}}^{\dagger} \hat{\mathcal{A}}}{2 S+1}\right)+\cdots\right] .
\end{aligned}
$$

Vê-se que a correção de ordem zero nada mais é do que a linearização dos operadores de spin com operadores do oscilador harmônico. Deste ponto, faz-se permitido utilizar todo o arsenal disponível sobre osciladores harmônicos à análise do problema linearizado em mãos. Já em primeira ordem, há uma correção adicional proporcional ao operador número normalizado $\hat{\mathcal{A}}^{\dagger} \hat{\mathcal{A}} /(2 S+1)$. O outro caso de grande interesse consiste, de fato, na aproximação de que os excitações bosônicas presentes no sistema não alteram significativamente a densidade média $\zeta$. Neste caso, os operadores de spin efetivos tornam-se

$$
\begin{aligned}
\eta & =\sqrt{(2 S+1)(1-\zeta)}, \\
\hat{S}_{+} & =\hbar \eta \hat{\mathcal{A}}^{\dagger}, \\
\hat{S}_{-} & =\hbar \eta \hat{\mathcal{A}} .
\end{aligned}
$$

Em ambos os casos, estados coerentes $|\lambda\rangle$ são autoestados convenientes para se estudar o problema [85], que satisfazem

$$
\hat{\mathcal{A}}|\lambda\rangle=\lambda|\lambda\rangle
$$

donde se vê que a remoção de uma excitação não produz mudança no estado, ou seja, são modos resilientes. Sua natureza, portanto, é consistente com hipóteses que mantém o número de ocupação médio muito próximos de $\zeta$ constante. Ora, isso é exatamente o que se espera nos dois casos de interesse listados anteriormente. Aqui vale a pena comentar que aplicações dos estados coerentes geralmente são restritas à Óptica Quântica, que não é necessariamente o caso dos modelos estudados nesta tese. Posto de outra maneira, a bosonificação permite o emprego do maquinário de Ótica Quântica em uma diversidade de sistemas complexos - como modelos epidêmicos, por exemplo - desde que sejam modelados por operadores de spin ou momento angular. 


\subsection{Tese}

Um dos fatos mais interessantes do processo de pesquisa científica lida com o desenrolar do processo criativo-investigativo. Muitas vezes, persegue-se determinado objetivo num projeto muito bem segmentado e definido. Porém, as experiências pessoais e técnicas sempre desempenham papel relevante: a bagagem técnica de cada pesquisador influencia os rumos adotados na investigação do tema. Um físico de partículas tende a buscar simetrias - e suas quebras - mesmo nos problemas mais elementares; um pesquisador de Mecânica Estatística desenvolve métodos que simplificam a descrição de muitos corpos e, nas horas vagas, costuma enxergar transições de fase nos problemas mais elementares; já o físico computacional desenvolve algoritmos e explora propriedades comuns aos sistemas físicos e aos sistemas computacionais, mesmo para os problemas mais elementares. Isso permite a formulação de diferentes interpretações para um mesmo fenômeno, uma vez garantida a consistência dos resultados mensuráveis.

O projeto de pesquisa inicial desta tese não foi diferente de seus pares. Consistia tão somente na investigação de materiais magnéticos nanoestruturados, no Laboratório de Modelagem de Sistemas Complexos, com o emprego de técnicas tradicionais de sistemas fortemente correlacionados. Contudo, a convivência diária e próxima com diferentes linhas de pesquisa e outros tipos de problemas e análises, acabaram por atrair a curiosidade científica sobre tais tópicos. A variedade de sistemas complexos compreende desde modelos epidemiológicos até materiais magnéticos nanoestruturados. Tais modelos não possuíam uma clara conexão em suas respectivas formulações teóricas, quando a pesquisa sobre o tema fora iniciado. A partir das evidências algébricas e computacionais, ficou patente que a teoria do momento angular sempre era matéria recorrente em diferentes sistemas complexos. Essa breve digressão serve apenas para contextualizar o leitor sobre a motivação que culminou no emprego da teoria de momento angular como ferramenta primária para a modelagem e pesquisa de sistemas complexos. Essa tese não foi fruto de planejamento prévio, sendo resultado da localização correta, com formação voltada para a investigação de simetrias e fenômenos cooperativos.

Todos os modelos e resultados reportados aqui são sumarizados da seguinte maneira. Primeiramente, após cuidadoso estudo sobre condições de contorno e topo- 
logia dos elementos componentes, formula-se operadores dinâmicos - sejam eles Hamiltonianos ou matrizes de transição - utilizando-se operadores de momento angular. Em seguida, propriedades algébricas e a natureza dos operadores são investigadas a fim de se determinar estratégias eficazes para sua resolução. Por fim, métodos de muitos corpos tradicionalmente restritos à Óptica Quântica ou sistemas fortemente correlacionados são empregados, reproduzindo resultados bem estabelecidos, e produzindo, principalmente, resultados originais. Estes últimos foram guiados pelas experiências e estudos prévios nas área de sistemas fortemente correlacionados, transições críticas de fase e teorias quânticas de campos. 


\section{3}

\section{Aplicações em modelos epidemiológicos}

Em segundo lugar, e mais importante, ninguém realmente entende o que é entropia, assim em um debate você sempre terá a vantagem.

- John von Neumann, sugestão a Claude Shannon

Durante a confecção desta tese, alguns dos nossos resultados mais significativos tiveram a investigação de modelos epidêmicos como ponto de partida. Dentre os resultados das Refs. [86, 87], mostramos que a análise de processos de disseminação de doenças são significativamente simplificadas considerando-se simetrias dos operadores de momento angular. Tal simplificação não se trata de aproximação, isto é, não há qualquer perda programática ou tampouco negligência do papel de flutuações estocásticas, sendo apenas uma simplificação do esforço analítico-computacional. Contudo, o resultado mais importante trata da descoberta de uma nova equação dinâmica para a entropia de Rényi. Essa equação vale para qualquer processo de Markov, que são predominantes em Física, inclusive processos de disseminação de doenças. Como muitas vezes acontece em Física, essa descoberta não fora prevista de antemão e tampouco sua interpretação decorreu de maneira imediata. Ao revisitar a problemática desses modelos sob a óptica da teoria de momento angular, encontramos um nicho extremamente fértil para a aplicação da teoria do momento angular de muitos corpos. Para começar a nossa discussão faz-se mister descrever em linhas gerais o que são os modelos epidêmicos, a fim de se estabelecer sua ligação com a teoria do momento angular.

A Epidemiologia é uma ciência quantitativa que estuda a distribuição e os 
determinantes dos estados de saúde, numa determinada população [88]. A outra função da Epidemiologia é transcrita como a análise adequada e fundamentada sobre evidências científicas robustas dos dados de Saúde supracitados. Cabe mencionar que essa análise muitas vezes repousa fora dos ramos tradicionais de Saúde Pública, empregando métodos estatísticos ou, como iremos apresentar ao longo deste capítulo, técnicas de Mecânica Estatística. Por esse motivo, as técnicas geralmente empregadas na Epidemiologia tem origem em outros ramos das Ciências e vice-versa. Exemplos podem ser facilmente encontrados como o estudo da propagação de vírus de computadores utilizando-se modelos da Epidemiologia [89].

Atualmente, as atividades da Epidemiologia são muito abrangentes [90] e envolvem aspectos demográficos, sociais e genéticos, objetivando o impacto de doenças não-comunicáveis como diabetes, por exemplo, e comunicáveis como sarampo. As análises decorrentes dessas atividade são, eventualmente, utilizadas por governantes e gerentes da Saúde Pública para determinar soluções ótimas que minimizam a ação da moléstia na população. Tais soluções assumem papel ainda mais relevante quando se considera a finitude dos recursos disponíveis. A disponibilidade de recursos torna-se particularmente mais sensível em economias emergentes, onde a presença de doenças comunicáveis como aids, tuberculose e malária são causas recorrentes de mortalidade na população.

A origem da Epidemiologia como ciência quantitativa moderna é inexoravelmente relacionada à transmissão de doenças comunicáveis entre seres humanos. Uma das ferramentas utilizadas consiste na confecção de modelos - doravante denominados modelos epidêmicos - que reproduzem os aspectos mais relevantes e fundamentais para a disseminação da doença comunicável sob investigação. O trabalho pioneiro de Kermack e McKendrick, desenvolvido em meados da década de 1920, é tido como uma das referências mais conhecidas da área [91]. Passado quase um século, não há evidência concreta que a transmissão de doenças pode ser considerado um problema fechado e resolvido. De fato, recentemente o mundo experimentou uma sequência inédita de epidemias ${ }^{1}$, as quais evidenciaram as deficiências dos modelos

\footnotetext{
${ }^{1}$ Define-se epidemia como a ocorrência excessiva de uma determinada doença numa dada população, em comparação com seu valor esperado. O conceito de epidemia é bastante intuitivo num cenário de surgimento de novas doenças contagiosas numa população susceptível. Ao mesmo tempo, também contempla a caracterização de epidemias de doenças comuns, não necessariamente comunicáveis ou estranhas ao ambiente como, por exemplo, gripe comum, diabetes, etc. Por este motivo, a caracterização como epidemia requer a localização geográfica, características da população, assim como a data. Mais ainda, o número de pacientes necessários para tal classificação depende também da população. Essa questão, apesar do teor burocrático, é deveras importante posto que a declaração de
} 
atuais.

Em 2003, a síndrome respiratória aguda grave (SARS) ganhou as manchetes. A doença infecciosa, causada pelo coronavírus, assemelha-se à gripe comum mas ocorre acompanhada de pneumonia, independente da faixa etária do paciente. O mecanismo de transmissão do coronavírus também é similar - contato com gotículas de saliva e secreção nasal provenientes de pacientes infectados - e afligiu aproximadamente 8 mil pessoas, com taxa de óbito em torno de 10\% [90]. A epidemia inciou-se na China mas espalhou-se rapidamente por outras regiões. Durante essa epidemia, os autores da Ref. [92] introduziram uma modelagem alternativa para estimar o número de reprodução efetivo $R_{t}$. O número de reprodução efetivo $R_{t}$ caracteriza a quantidade de novas incidências de determinada doença na população, no tempo $t$, a partir de um único indivíduo infectado. Ainda que sua determinação seja imprecisa, essa quantidade provê uma estimativa para a evolução da doença. Essa nomenclatura tem origem na caracterização de 1930 de Kermack, que define o número de reprodução $R_{0}$ no início de uma epidemia. ${ }^{2}$ Um fato bastante curioso na abordagem desenvolvida na Ref. [92] foi a estimativa de $R_{t}$ a partir de dois comportamentos distintos: um calculado a partir de uma região da China onde a doença se espalhava de maneira lenta; e outra contribuição onde a doença se espalhava de modo muito acentuado. Essa estimativa ad hoc avaliou corretamente que a epidemia estava sob controle, e que os métodos de quarentena empregados pelo governo chinês eram eficazes.

Em 2009 foi a vez da gripe causada por um novo tipo de vírus influenza A H1N1 (H1N1/09), popularmente conhecida como gripe suína. Ao contrário da gripe comum, as manifestações mais severas da doença atingem pessoas saudáveis de todas as faixas etárias, levando eventualmente a rápida deterioração das condições respiratórias. A Organização Mundial de Saúde (OMS) declarou o surto deste vírus uma pandemia, que persistiu até meados de 2010. No Brasil, o histórico das influenza

epidemias geralmente permite a liberação de recurso financeiros e humanos por parte de organismos governamentais. Daí a necessidade contínua de melhor para se estabelecer se dado surto de doença é ocorrência normal ou epidemia.

${ }^{2}$ Deve causar estranheza ao leitor o emprego da variável temporal $t$ como índice de $R_{t}$ e a definição de $R_{0}$ e $R_{t}$ como quantidades independentes. Cabe aqui comentar que, ao iniciar as pesquisas na área, achei o conceito em si muito inadequado pois sempre considerei populações com tamanho finito. A finitude da população naturalmente impõe limites ao crescimento da doença, o que resulta numa dependência temporal da taxa de reprodução. Porém, essa hipótese não é sempre observada, especialmente quando a doença fica restrita a um número muito pequeno de indivíduos frente ao restante da população. Nesse caso, $R_{0}$ é um bom parâmetro. Claro, existem diversos outras causas que efetivamente desviam o valor $R_{t}$ de $R_{0}$ e cujo efeito não é adequadamente capturada pelo modelo como, por exemplo, a introdução de quarentena aos doentes, pânico da população, etc. Na prática, ainda ocorrem discussões relevantes sobre as duas grandezas, como pode ser visto na Ref. [93]. 
tipo A (H1, H5, etc) e B é apresentado na Figura 3.1a. Os gráficos da Figura 3.1b apresentam dados mais detalhados relativo ao ano de 2016, período no qual se observou uma branda epidemia de influenza tipo A, compreendida entre os meses de Março até Maio. Desde a pandemia de 2009, o comportamento quasi-periódico para ocorrência de epidemias tem sido reproduzido também em outros países.

Já entre 2013 e 2015, houve uma grave epidemia de febre hemorrágica causada pelo vírus ebola Zaire ebolavirus, no oeste da África [95]. A doença é caracterizada por forte febre, pelo surgimento de hemorragias pelo corpo e pelo alto índice de mortalidade [96]. Sua transmissão se dá por meio do contato direto com fluídos corporais de pacientes infectados (sangue, saliva, vômito, fezes, etc), ficando assim primariamente restrita aos agentes de saúde e familiares [97]. Apesar de não ser transmitido por vias aéreas, a agressividade dos sintomas causados pela doença ocasionaram a morte de aproximadamente 11 mil pessoas [97]. Atualmente, a epidemia do vírus Zika encontra-se em andamento, ao qual se atribui como evento causador da microcefalia em recém-nascidos, uma deformidade neo-natal caracterizada pela redução anormal do crânio e cérebro [98]. Os mecanismos de transmissão dessa doença ocorre majoritariamente por meio de vetores, mosquitos infectados da espécie Aedes - tanto Aedes aegypti como Aedes albopictus - que também transmitem os vírus da dengue e chikungunya. Porém, outros mecanismos de transmissão estão sendo atualmente investigados como a transmissão por meio de transfusão sanguínea, relações sexuais e de mãe para filho, durante a gravidez.

No que segue, descrevemos os modelos epidêmicos mais empregados considerando-se tão somente dois estados de saúde, infectado e susceptível, numa população de tamanho finito. Por questões pedagógicas, discutimos brevemente a metodologia mais tradicional, denominada abordagem compartimental, que descreve a evolução temporal de doenças infecciosas por meio de equações diferenciais parciais, onde há uma forte assunção a respeito da homogeneidade da população. Alternativamente, a abordagem baseada em agentes, foco principal deste capítulo, aborda questões que envolvem a heterogeneidade da população e correlações entre os diferentes indivíduos. 


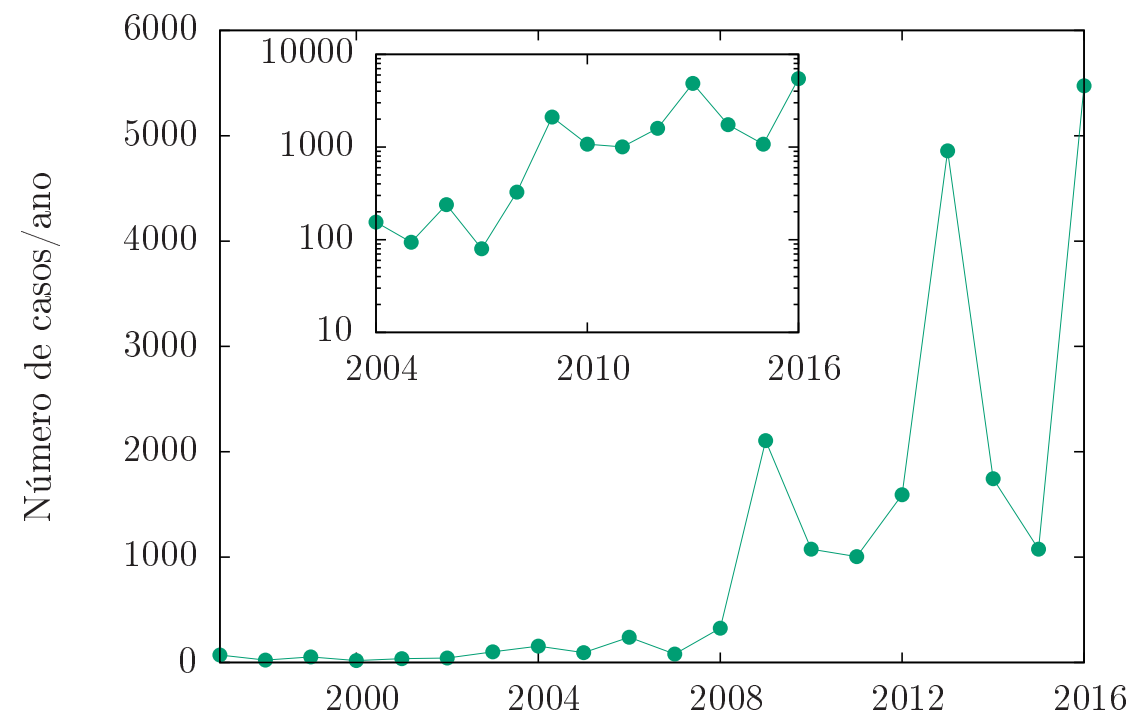

a)

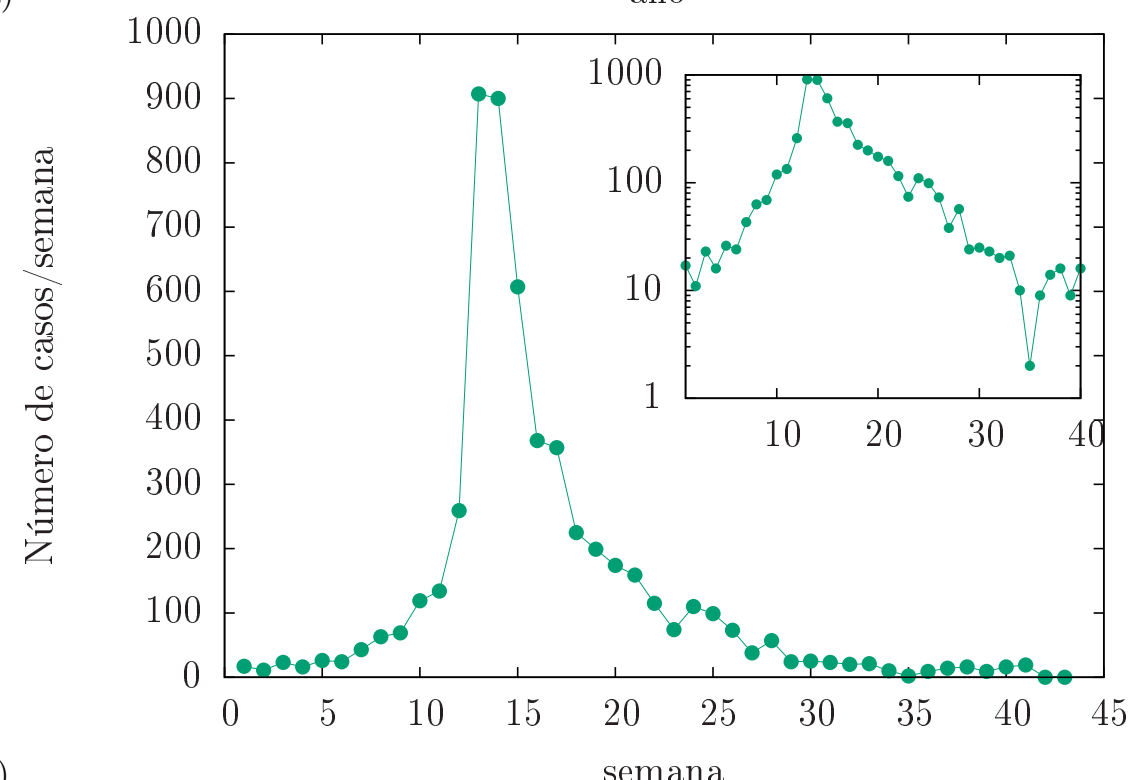

Figura 3.1: Número de casos de influenzas atípicas no Brasil ao longo do tempo. Fonte: Organização Mundial da Saúde [94]. a) Os novos casos confirmados laboratorialmente das diversas subvariedades de influenza tipo A (H1, H5, etc) e do tipo B são contabilizadas anualmente. A epidemia inicial de 2009 (H1N1) foi muito mais branda que as seguintes, apesar do desenvolvimento e uso de vacinas para algumas variedades. No inset, os mesmos dados são apresentados num gráfico semi-log, de onde se vê que durante uma epidemia, o número de casos cresce exponencialmente. b) A epidemia mais recente, em 2016, assim como as anteriores possui breve duração e crescimento/decaimento assimétrico ao longo das semanas. No inset, observa-se que o crescimento que origina a epidemia cresce mais rapidamente que a função linear na escala logarítmica. 


\subsection{Modelos epidêmicos}

De modo geral, modelos epidêmicos descrevem a evolução temporal de uma ou mais doenças comunicáveis em uma determinada população. ${ }^{3}$ Tais modelos levam em conta a cadeia de infeção da doença [90], a qual pode ser descrita como o produto da interação adequada do patógeno e hospedeiro (indivíduo da população), mediante a existência de um ou mais processos de transmissão, considerando-se os vínculos ambientais. Aqui, o termo patógeno é empregado de modo genérico e faz referência ao agente que é transmitido (infecção), isto é, pode ser um vírus, uma bactéria ou parasita.

Vale a pena mencionar que nem sempre a infecção (transmissão do patógeno) propicia condições favoráveis para o desenvolvimento da doença ou mesmo a multiplicação do agente patogênico já instalado. Dado esta observação, denomina-se susceptível, abreviado pela letra $S$, aquele indivíduo capaz de desenvolver a doença em sua plenitude, isto é, tornar-se infectado, $I$, e capaz de comunicar a doença a terceiros. Para fins de definição, também existe a categoria de indivíduos exposto ao patógeno, $E$, e aqueles que se recuperam da infeção ou adquirem imunidade, $R$. As categorias $(S, E, I$ e $R$ ), que dividem os estágios de uma doença nos indivíduos de uma população, são denominados de estados de saúde e constituem elementos primários para a formulação de modelos epidêmicos. O número de diferentes estados de saúde $q=2,3, \ldots$ depende das particularidades da moléstia (ver Figura 3.2) e caracteriza o modelo.

A definição do número de estados $q$ não é suficiente para descrever a dispersão da doença na população. Essa evolução temporal exige o conhecimento preciso do mecanismo de transmissão, que no caso de seres vivos geralmente envolve tanto aspectos dinâmicos quanto biológicos (microscópicos, bioquímicos e genéticos) e sociais, por meio da rede de relacionamento do indivíduo infectado com seus pares. Claro, o intuito dessa capítulo não é aprimorar um tratado sobre estes temas em suas respectivas áreas do Saber - que certamente deixaria a desejar frente à formação acadêmica do autor -, mas prover as ferramentas essenciais para a modelagem matemática do tema. Desse modo, negligenciamos os detalhes biológicos e soci-

\footnotetext{
${ }^{3} \mathrm{~A}$ introdução de mais de uma doença leva em conta o inter-relacionamento de doenças crônicas e infecções oportunistas, como no caso da síndrome da imunodeficiência adquirida (aids) e tuberculose. A dinâmica conjunta das doenças dificulta substancialmente o desenvolvimento de abordagens analíticas, devido à introdução de mais graus de liberdade no sistema.
} 

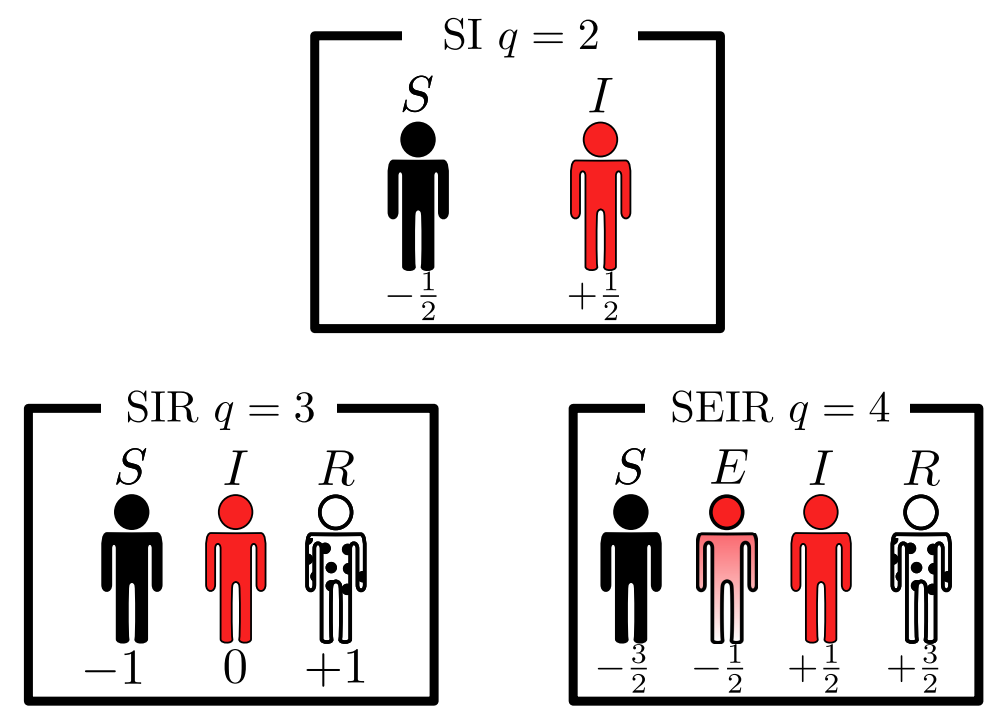

Figura 3.2: Estados de modelos epidêmicos. Os diferentes estados de saúde presentes num dado modelo epidemiológico é determinado pelo número $q$, com $q=2, \ldots$ inteiro positivo. Na gravura, o estado de saúde do indivíduo é caracterizado pela letra, por uma cor (para facilitar a visualização) e, mais importante, por um número. O caso $q=1$ não apresenta nenhuma mudança de estado então é descartado. O caso $q=2$ estabelece apenas dois estados, susceptível $S$ e infectado $I$. O indivíduo $S(I)$ também é caracterizado pelo número $-1 / 2(+1 / 2)$ em clara analogia com o número quântico azimutal de um férmion com spin $s=1 / 2$. O caso $q=3$ possui adicionalmente o estado recuperado $R$, enquanto o caso $q=4$ possui também o estado exposto $E$. Analogamente, para $q=3(q=4)$ faz-se referência ao número quântico azimutal de um bóson (férmion) de spin $s=1$ $(s=3 / 2)$.

ais, excetuando-se os casos onde a dinâmica, seja ela determinística ou estocástica, conflita com preceitos envolvidos nas hipóteses biológicas ou sociais.

Grosso modo, divide-se a transmissão em duas categorias abrangentes. Na transmissão direta, o patógeno é transferido de um indivíduo infectado a um indivíduo susceptível, sem interferência de fatores externos ou ambientais. Aqui enquadram-se doenças sexualmente transmissíveis, por meio da troca de gotículas de saliva ou fluidos corporais a curta distância, transfusões sanguíneas, etc. Já os mecanismos indiretos requerem a intervenção de fatores ou agentes externos como, por exemplo, mosquitos ou alimentos contaminados. Neste caso, tais elementos externos requerem uma descrição dinâmica própria, que em determinado momento se acopla sinergicamente com a dinâmica de transmissão, habilitando ou favorecendo a disseminação do patógeno pela população.

Para tornar nossa discussão mais concreta, concentramos o texto a seguir sobre a dinâmica de disseminação do modelo SIS, em uma população composta 
de $N$ indivíduos distintos (Figura 3.3). Para fins de notação, a enumeração dos indivíduos utiliza índices latinos, $k=0,1, \ldots, N-1$. Cada indivíduo pode assumir dois estados de saúde mutuamente exclusivos, $S$ ou $I$, ao longo do tempo, ou seja, $q=2$.

Os estágios de evolução da doença expressa pelo modelo SIS segue a seguinte cadeia $S \rightarrow I \rightarrow S$, isto é, um sujeito $S$ pode ser infectado e eventualmente retorna a seu estado de saúde normal. A recuperação de um indivíduo infectado, espontaneamente ou como produto de tratamento externo, possui probabilidade $\gamma$ durante um intervalo de tempo $\delta t$. Uma maneira bastante popular ${ }^{4}$ de se expressar essa transição decorre do formalismo estequiométrico [21],

$$
I \rightarrow \gamma S+(1-\gamma) I
$$

Caso $\gamma$ se anule, o modelo recebe a denominação SI, onde a doença, uma vez contraída, não permite a possibilidade de cura, como a aids por exemplo. Ademais, um indivíduo $I$ transmite o patógeno para um indivíduo $S$ com probabilidade $\beta^{\prime}$, durante o intervalo de tempo $\delta t$, se assumido o contato adequado entre os dois. Por uma questão de conveniência, convém escrever $\beta^{\prime} \equiv \beta / N$. Considerando-se as transições que envolvem apenas dois indivíduos complementares, tem-se

$$
S I \rightarrow \frac{\beta}{N} I I+\gamma S S+\left(1-\gamma-\frac{\beta}{N}\right) S I
$$

Existem três fatos relevantes no que foi exposto acima que merecem discussões adicionais. Primeiro, as transições que envolvem dois corpos (transmissão) também levam em conta as transições que envolvem apenas um corpo (cura). Essa observação às regras torna-se mais importante ainda dado que a transição $S I \rightarrow S I$ possui probabilidade complementar à soma das transições restantes. Segundo, a Eq. (3.2) assume que os indivíduos $S$ e $I$ interagem adequadamente de maneira a permitir a transmissão do patógeno. Por conseguinte, a Eq. (3.2) não leva em conta as particularidades da rede de relacionamento reais de cada ente da população. Por fim, todas as probabilidades acima dependem do valor do intervalo de tempo $\delta t$. Voltaremos à essa questão mais adiante, ao lidar com o modelo estocástico baseado em agentes.

Apesar de ser bastante simples, a solução exata do modelo SIS só é conhecida

\footnotetext{
${ }^{4} \mathrm{~A}$ popularidade das equações estequiométricas é produto de sua analogia química.
} 

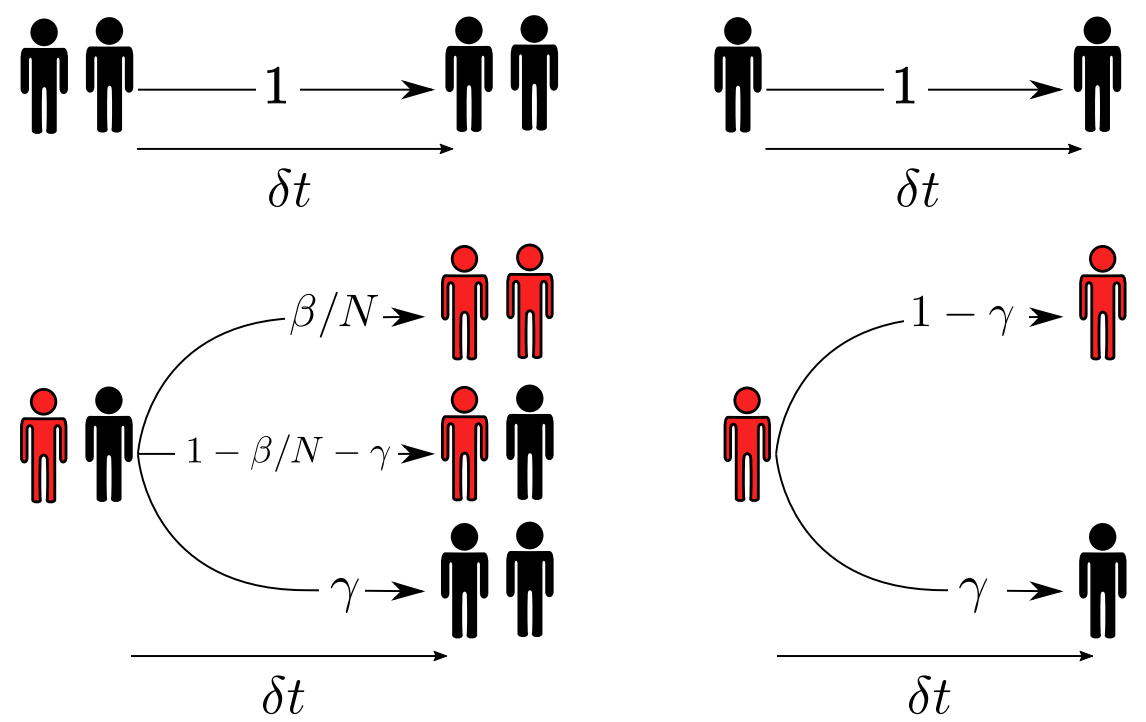

Figura 3.3: Quatro transições de estado no modelo SIS, durante o intervalo de tempo $\delta t$. As transições de "um corpo" são listadas do lado direito e expressam as equações estequiométricas $S \rightarrow S$ e $I \rightarrow(1-\gamma) I+\gamma S$, respectivamente. De acordo, $\gamma$ é a probabilidade de um indivíduo infectado retornar espontaneamente ao estado susceptível. As gravuras do lado esquerdo representam graficamente as transições de "dois corpos", com suas respectivas equações estequiométricas $S+S \rightarrow S+S$, e $I+S \rightarrow \gamma S S+(\beta / N) I I+(1-\gamma-\beta / N) I S$, onde $\beta / N$ é a probabilidade de que ocorre a transmissão do patógeno para um indivíduo previamente susceptível. Enfatizamos que transições duplas não são consideradas devido à aproximação poissoniana sobre $\delta t$.

quando a população apresenta elevada homogeneidade, com $N \gg 1$. Sua descrição matemática comporta três abordagens, distintas entre si de acordo com o grau de refinamento das correlações entre entes constituintes, a saber, compartimental (macroscópica), estocástica (mesoscópica) e, por fim, estocástica baseada em agentes (microscópica). A abordagem compartimental possui grande difusão em Epidemiologia, de modo que consideramos necessária sua discussão para expor ao leitor o estado corrente da área, suas fraquezas e também pontos fortes. ${ }^{5}$ As comparações com os mesmos pontos na abordagem baseada em agentes são os elementos que habilitam a análise adequada das vantagens e desvantagem de cada metodologia, no estudo de processos de disseminação de doenças.

\footnotetext{
${ }^{5}$ Convém comentar que exposições breves, sem a devida profundidade conceitual, tendem a ser enviesadas. Apesar de não ser o intuito desta obra, é palpável que o autor acabe por introduzir um viés na narrativa dada a superficialidade descritiva de uma das abordagens. A fim de mitigar a passagem errônea de mensagem, vale a pena explicitar que o desenvolvimento da abordagem compartimental ainda se situa como ferramenta primária na análise e predições de epidemias, especialmente naquelas que ocorrem em largas escalas [21, 99].
} 


\subsection{Abordagem determinística compartimental}

Como mencionado anteriormente, existem diferentes graus de minúcia nas diferentes abordagens de modelos epidêmicos, em relação às particularidades dos entes da população. A vertente mais simples classifica os elementos da população em $q$ categorias, organizadas de acordo com os $q$ estados de saúde disponíveis ao modelo, desconsiderando-se quaisquer particularidades individuais (Figura 3.4).
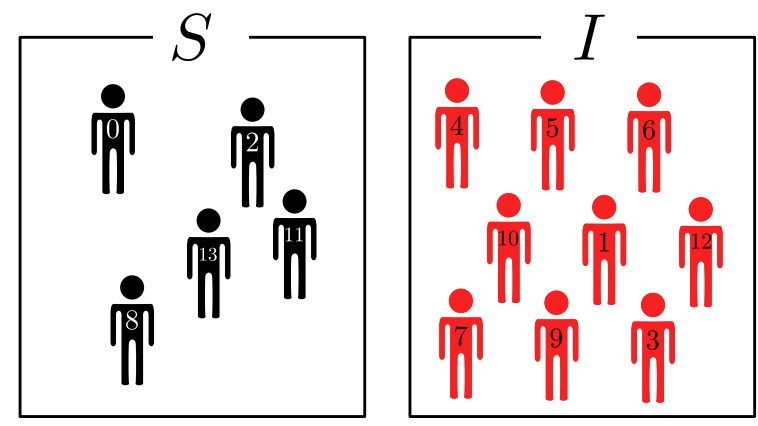

Figura 3.4: Agrupamento de indivíduos de uma determinada população de tamanho $N=14$ no modelo SIS. Cada ente é enumerado pelo inteiro $k=0,1, \ldots, N-1 \mathrm{e}$, a partir de seu estado de saúde, encontra-se agrupado ou no compartimento $S$ (susceptível) ou no compartimento $I$ (infectado). A compartimentalização de estado ignora as características individuais, como o identificador $k$ bem como as estruturas de relacionamento interno entre os membros da população. Na dinâmica compartimental, indivíduos são retirados de um compartimento e colocados no outro de acordo com as taxas de infecção $\beta$ e cura $\gamma$.

A denominação "abordagem compartimental" decorre desse agrupamento, cuja principal hipótese é a inexistência de correlações internas na população, em outras palavras, qualquer indivíduo representa muito bem o indivíduo típico da mesma população. Essa hipótese ${ }^{6}$ é conhecida como hipótese de mistura aleatória $[13,23]$. Logo, médias também são estatísticas muito representativas. Dentre as estatísticas relevantes, o número $n_{r}(t)(r=S, I, R, E \ldots)$ de diferentes indivíduos dentro da categoria $r$ (compartimento) serve como variável para explorar a dinâmica global da doença. No caso do modelo SIS, existem apenas dois compartimentos e, portanto, $n_{S}(t)$ e $n_{I}(t)$, e que satisfazem ao vínculo

$$
n_{S}(t)+n_{I}(t)=N(t)
$$

No caso de populações com tamanho fixos, $N(t) \equiv N$. Apesar de ser perfeitamente possível introduzir dinâmicas populacionais de nascimento e morte, efetivamente

\footnotetext{
${ }^{6}$ A hipótese de mistura aleatória também é conhecida como aproximação de campo médio. Contudo, essa denominação é muitas vezes conflitante com a aproximação de campo médio assim formulada em Física Estatística, apesar da equivalência em alguns cenários. Por esse motivo, adotamos a nomenclatura da mistura aleatória.
} 
removendo o caráter paramétrico de $N$, estes não serão tratados aqui.

A hipótese de mistura aleatória permite o desenvolvimento de equações nãolineares que objetivam a reprodução da evolução do número de infectados $n_{I}(t)$ e susceptíveis $n_{S}(t)$. Destaca-se que a investigação das soluções deste sistema promove uma visão geral do sistema saúde-doença, dai seu valor quantitativo imediato. No caso do modelo SIS com $N$ fixo, o sistema de equações reduz-se a apenas uma única equação diferencial $[13,23,100]$

$$
\frac{d n_{I}}{d t}=\frac{\beta}{N} n_{I}\left(N-n_{I}\right)-\gamma n_{I}
$$

Essa expressão possui uma interpretação física bastante intuitiva. A população de infectados aumenta com o número "colisões" entre infectados e susceptíveis por unidade de tempo, dado pelo termo $\beta n_{I}\left(N-n_{I}\right)$, e decai proporcionalmente ao número de infectados, ou seja, $-\gamma n_{I}$. Dividindo-se a Eq. (3.4) por $N$ e definido-se a densidade $\rho(t)=n_{I}(t) / N$, obtém-se

$$
\frac{d \rho}{d t}=\beta \rho(1-\rho)-\gamma \rho
$$

que escala com o tamanho da população, como apontam os Autores da Ref. [21]. Essa observação é importante pois as equações compartimentais são muito mais robustas no limite termodinâmico, onde $N \rightarrow \infty \operatorname{com} n_{I} \gg 1$ tal que a razão $n_{I} / N$ converge para $\rho$. Por se tratar de uma equação de Bernoulli, calcula-se a solução da Eq. (3.4) por meio da transformação de variável $z=1 / \rho$ :

$$
\frac{d z}{d t}=-\beta \rho_{\mathrm{eq}} z+\beta,
$$

$\operatorname{com} \rho_{\text {eq }}=1-\gamma / \beta$. Para a condição inicial $\rho(0)=\rho_{0}>0$, obtém-se (Figura 3.5)

$$
\rho(t)=\frac{\rho_{\mathrm{eq}}}{1+\rho_{\mathrm{eq}} \mathbb{C} \mathrm{e}^{-\beta \rho_{\mathrm{eq}} t}}, \quad \mathbb{C}=\frac{1}{\rho_{0}}-\frac{1}{\rho_{\mathrm{eq}}},
$$

de onde se determina o tempo característico $\tau=|\beta-\gamma|^{-1}$.

Atualmente, generalizações da Eq. (3.4) são amplamente utilizadas [1, 13, 58, 89, 101]. O objetivo destas generalizações centra-se em corrigir a hipótese de mistura aleatória, levando em consideração aspectos das estruturas sociais características da população. Essa correção ad hoc é bastante intuitiva e é introduzida pela introdução do coeficiente $\zeta$ :

$$
\frac{d \rho}{d t}=\beta \zeta \rho(1-\rho)-\gamma \rho
$$




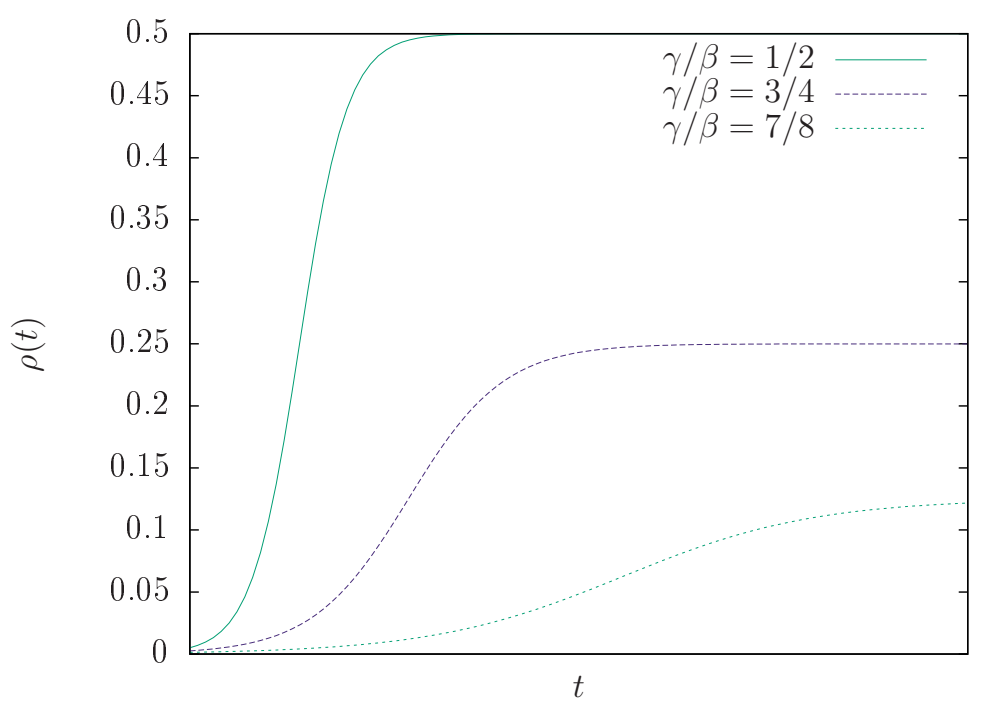

Figura 3.5: Soluções típicas da Eq. (3.4) com $\rho_{0}=0.01$ para três valores distintos da razão $\gamma / \beta$. A densidade de equilíbrio é dada por $\rho_{\mathrm{eq}}=1-\gamma / \beta$.

Aqui, $\zeta$ carrega a informação sobre a estrutura social por meio do valor numérico, referente à quantidade de interações típicas por indivíduo por unidade de tempo. Existem ao menos dois valores amplamente utilizados: $\zeta=\left\langle k^{2}\right\rangle /\langle k\rangle$ e $\zeta=\langle k\rangle / N$. Os valores médios $\langle k\rangle$ e $\left\langle k^{2}\right\rangle$ são respectivamente o primeiro momento do número de coordenação, melhor conhecido como grau médio, e o segundo momento do número de coordenação. ${ }^{7}$ Na prática, essa correção devido à estrutura social e de relacionamento equivale à transformação $\beta \rightarrow \beta \zeta$, de modo que a solução torna-se

$$
\rho(t)=\frac{\rho_{\mathrm{eq}}}{1+\rho_{\mathrm{eq}} \mathbb{C e}-\zeta \beta \rho_{\mathrm{eq}} \cdot t}, \quad \mathbb{C}=\frac{1}{\rho_{0}}-\frac{1}{\rho_{\mathrm{eq}}},
$$

onde $\rho_{\text {eq' }}=1-(\gamma / \beta \zeta)$.

Apesar da solução analítica (3.7), convém discutir alguns pontos relevantes que foram propositalmente omitidos até agora. Primeiramente, a razão $\beta / \gamma$ coincide com a definição de $R_{0}$, cujo uso primário avalia a extinção da epidemia. De fato, para $R_{0}<1$ a epidemia decai com um tempo característico $\tau=\left(R_{0}^{-1}-1\right)^{-1}$, enquanto para $R_{0}>1$ a epidemia estabiliza-se com densidade $\rho_{\text {eq }}=1-R_{0}^{-1}$. Contudo, quando $\beta=\gamma$ ou $R_{0}=1$, a solução da Eq. (3.4) é uma lei de potência

$$
\rho(t)=\frac{\rho_{0}}{1+\beta \rho_{0} t},
$$

cuja principal característica encontra-se na ausência de um tempo característico $\tau$.

\footnotetext{
${ }^{7}$ Uma breve exposição sobre teoria de redes encontra-se no Apêndice 1.
} 
Sob estas circunstâncias, a epidemia sobrevive por longos períodos de tempo. Em particular, se $\beta \rho_{0} \rightarrow 0$, então o tempo de meia-vida $t_{1 / 2}=\left(\beta \rho_{0}\right)^{-1}$ diverge.

No equilíbrio, a Eq. (3.5) torna-se

$$
\left.\frac{d \rho}{d t}\right|_{\mathrm{eq}}=0=\beta \rho(1-\rho)-\gamma \rho,
$$

a partir da qual se determina a densidade de equilíbrio trivial $\rho_{\mathrm{eq}}=0$ se $\gamma \geqslant \beta$ ou $\rho_{0}=0$; e a densidade não-trivial $\rho_{\text {eq }}=1-\gamma / \beta$ quando $\beta>0 \operatorname{com} \gamma / \beta<1$. Resta determinar se estes regimes são estáveis. Isto pode ser feito mediante à adição de uma perturbação $\delta \rho(t)$ à solução $(3.7), \rho^{\prime}=\rho+\delta \rho$. Para simplificar a análise, convém introduzir a perturbação após um tempo $T$, já próximo ao equilíbrio, pois $\rho(T) \approx$ $\rho_{\text {eq. }}$. Num primeiro momento, consideramos apenas pequenas perturbações tal que $|\delta \rho / \rho| \ll 1$, agrupando as correções como uma série de potências. Substituindo $\rho^{\prime}$ na Eq. (3.4) e considerando apenas os termos lineares em $\delta \rho$, temos

$$
\left(\frac{d}{d t}\right) \delta \rho=-\beta \rho_{\mathrm{eq}} \delta \rho,
$$

com solução $\delta \rho=\delta \rho_{0} \mathrm{e}^{-\beta \rho_{\mathrm{eq}}(t-T)}$, onde $\delta \rho_{0}$ é a amplitude da perturbação inicial. Devido à positividade da constante de $\beta \rho_{\text {eq }}>0$, conclui-se que a solução $\rho_{\text {eq }}=1-$ $\gamma / \beta$ é estável, pois pequenas perturbações sobre a densidade do sistema rapidamente retornam ao equilíbrio, como ilustra a Figura 3.6.

Contudo, existe uma patologia quando $\beta \rho_{e q} \rightarrow 0$, tanto para $\gamma / \beta \rightarrow 1$ quanto para $\beta \rightarrow 0$. Nesse caso patológico, a perturbação retira o sistema do regime de equilíbrio $\rho_{\mathrm{eq}}$ e o joga, efetivamente, no regime $\rho_{\mathrm{eq}}+\delta \rho$, exigindo um longo intervalo de tempo até seu retorno ao regime de equilíbrio natural. Do ponto de vista prático, isto significa que pequenas ações de redução da densidade de infectados, mas recorrentes, eventualmente produzem a erradicação da doença caso $\beta \rightarrow 0$. A questão principal, portanto, envolve quais as condições necessárias para que $\beta \ll 1$, a saber, o intervalo de tempo utilizado para se mensurar ou estimar a taxa $\beta$ e o tamanho da população amostrada.

O fato do sistema apresentar um estado absorvente em $\rho=0$ também deve ser levado em consideração quando sob ação de perturbações com amplitudes arbitrárias. Por arbitrária entende-se amplitudes que compreendem deslocamentos na densidade desde seu valor mínimo $\rho=0$ até seu valor máximo $\rho=1$. Destaca-se 
que as pertubações que produzem $\rho \rightarrow 0$, efetivamente deslocam o sistema de um ponto fixo do sistema dinâmico $\left(\rho_{e q} \neq 0\right)$ e para outro $\left(\rho_{e q}=0\right)$. Se interpretarmos perturbações como eventos aleatórios, concluímos que flutuações na densidade podem levar um regime diferente do valor esperado $\rho=1-\gamma / \beta$, dependendo dos valores de $\gamma / \beta$, a frequência de ocorrência das perturbações e de suas amplitudes.

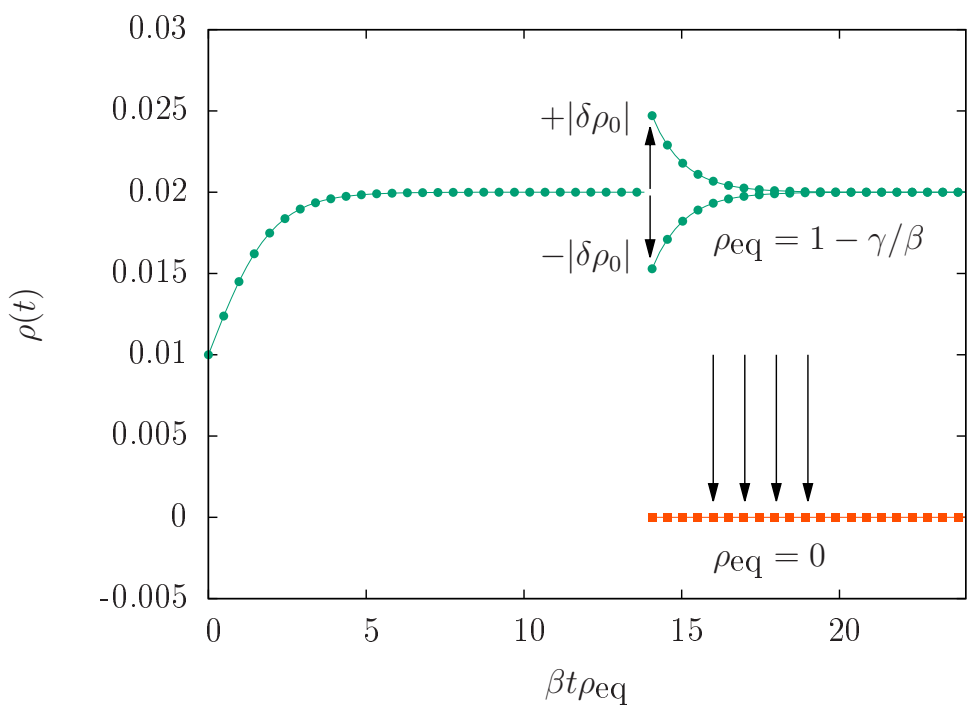

Figura 3.6: Estabilidade sob ação de perturbação no equilíbrio para o modelo compartimental SIS, $\operatorname{com} \beta=2$ /unid. tempo e $\gamma / \beta=0.98$. A densidade de infectados evolui de acordo com a solução expressa na Eq. (3.7) até o tempo escalado $\beta t \rho_{e q}=14$, quando uma perturbação de intensidade $\left|\delta \rho_{0}\right|=\rho_{e q} / 4$ é imposta ao sistema ( os casos $\delta \rho_{0}>0$ e $\delta \rho_{0}<0$ são ilustrados simultaneamente). Em ambos os casos, a densidade do sistema retorna exponencialmente ao equilíbrio $\rho_{\text {eq }}=0.02$. Cada unidade de tempo escalado corresponde a $\left(\beta \rho_{e q}\right)^{-1}=25$ unid. de tempo real utilizado na mensuração de $\beta$. A linha com quadrados (laranja) indica a ação de uma perturbação com intensidade igual à densidade $\rho_{e q}$. Ao contrário dos casos anteriores, o sistema permanece no regime com $\rho=0$.

Devido à equivalência entre os indivíduos da população, flutuações nas descrições compartimentais são automaticamente descartadas. Naturalmente, uma população real possui particularidades locais que influenciam a disseminação de uma doença. Por exemplo, acesso a aeroportos e outros meios de transporte aumentam a eficiência da transmissão de doenças para longas distâncias; contaminação de curtas distâncias são mais frequentes entre membros da mesma família e local de trabalho; etc. Quando as doença já encontra-se em equilíbrio estável ou quando a população possui elevado grau de similaridade, as abordagens compartimentais tendem a produzir resultados compatíveis com aqueles esperados intuitivamente. Por outro lado, populações finitas e com elevado grau de variabilidade individual não satis- 
fazem à hipótese de mistura aleatória. Esses casos são recorrentes nos estudos de novas doenças como febre hemorrágica e exigem abordagens que levem em conta as estruturas sociais e demográficas da população [22].

\subsection{Abordagem estocástica baseada em agentes}

A Epidemiologia atual contempla a variabilidade da população na análise da distribuição da doença e seus efeitos. Como consequência, o papel de flutuações, as estruturas sociais e informações geográficas não podem ser desprezadas ao se tratar da matéria de disseminação de doenças. Por outro lado, a inclusão de tais informações inexoravelmente impossibilita o emprego das hipóteses requeridas pela abordagem compartimental [100]. Torna-se, portanto, necessário abrir mão da modelagem através de equações diferenciais determinísticas e considerar formulações por meio de processos estocásticos [22]. ${ }^{8}$

Processos estocásticos são modelos probabilísticos que descrevem a evolução espaço-temporal (ou sobre alguma outra variável relevante) de determinado fenômeno $[20,53,102,103]$. O objetivo primordial do processo estocástico consiste na determinação precisa e completa da distribuição de probabilidade, ou função densidade de probabilidade no caso de variáveis contínuas, de um sistema a partir um conjunto de regras mais simples. Em geral, essas regras são ponderadas por probabilidades e envolvem apenas parte do sistema em cada iteração, caso o sistema contenha vários graus de liberdade. Quando as regras são construídas utilizando-se iterações consecutivas, como intervalos de tempos consecutivos, então o processo é classificado como um processo de Markov [20]. O exemplo mais popular é o problema do caminhante aleatório unidimensional [53]. Neste problema, um ponto material pode transladar no espaço por uma distância $\delta x$ para a direita ou esquerda com iguais chances a cada intervalo de tempo $\delta t$. Observe que a probabilidade de ir para esquerda ou direita descreve uma regra bastante simples: a posição corrente (estado) $x$ pode incrementar (diminuir) por $\delta x$ (outro estado) com probabilidade $1 / 2$. Essas regras descrevem a probabilidade de transição $p(x \rightarrow x \pm \delta x)=1 / 2$, e neste caso particular tem mesma amplitude.

\footnotetext{
${ }^{8}$ Vale a pena notar que formulações estocásticas não implicam no descarte de equações diferenciais. O exemplo mais famoso é a equação de Fokker-Plank para o movimento browniano [102], onde a expressão da evolução temporal da função densidade de probabilidade ocorre por meio de uma ferramenta determinista.
} 
Intuitivamente, a estimação das probabilidades de transição é mais simples de se medir do que a distribuição de probabilidade do problema, $P(x, t)$. O motivo é que, dependendo do intervalo de tempo $t=L \delta t$, existem diversos caminhos diferentes e que produzem a posição final $x=m \delta x$ do caminhante. Processos estocásticos exploram exatamente essa noção e tentam resolver um problema complicado - determinação de $P(x, t)$ - por meio de um conjunto finito de probabilidades de transição, que são mais simples de serem estimadas.

Ora, relacionando-se a definição de processos estocásticos com modelos epidêmicos, nota-se que as mudanças dos estados de saúde dos entes da população descrevem transições. Mais ainda, a estimação da chance de transmissão do patógeno ou de recuperação individual certamente é mais simples do que a determinação da distribuição de probabilidade da doença na população de tamanho $N$, no tempo t. Apesar disso, a mera identificação das probabilidades de transição individuais não é suficiente para se caracterizar ou analisar o impacto de uma epidemia sobre uma população real. Isso é especialmente verdadeiro quando a população afetada é heterogênea, possui número reduzido de indivíduos em situação de risco ou a disseminação da doença encontra-se ainda em seu estágio inicial.

Sob estas circunstâncias, a OMS sugere a coleta de informações (idade, sexo, geolocalização, local de trabalho, familiares, contatos, datas do surgimento da doença, etc) de cada pessoa infectada. Essa prática, denominada de rastreamento, tem dois objetivos: estabelecer a cadeia temporal de transmissões e, com sorte, o primeiro paciente infectado; esquematizar a rede de relacionamento dos infectados, priorizando cuidado e tratamentos aos entes mais próximos do infectado, devido ao maior risco de infecção. Como rastreamento dos infectados e cuidados clínicos individuas são cruciais no planejamento estratégico da OMS para conter ou limitar o avanço de incidências de doenças emergentes (ebola, etc), há interesse em modelos que levem em conta a heterogeneidade inerente da população para descrever processos de disseminação de doenças. Tais modelos recebem a denominação de modelos epidêmicos baseado em agentes, do inglês agent based epidemic models (ABEM). ${ }^{9}$

ABEM são modelos matemáticos que expressam a evolução de doenças infecciosas entre um número finito $N$ de agentes, ao longo do tempo. Para esse propósito,

\footnotetext{
${ }^{9}$ A fim de evitar confusões de nomenclatura, utilizamos o termo agente como sinônimo de indivíduo até o final do capítulo. Postergou-se o emprego do termo agente pois essa denominação é geralmente atribuída ao patógeno em Epidemiologia.
} 
os agentes são identificados univocamente por um número inteiro $k=0,1, \ldots, N-1$, enquanto o relacionamento ${ }^{10}$ entre os diferentes são mapeados por meio da matriz de adjacência $A(N \times N)$. Por definição, os elementos da matriz $A_{i j}=1$ se o $j$-ésimo agente possui conexão com o $i$-ésimo agente, nulo caso contrário. Desse modo, a heterogeneidade surge naturalmente, pois as particularidades dos agentes são contempladas por meio da matriz $A$, distanciando os modelos baseados em agentes das hipóteses dos modelos compartimentais [13, 100]. Formalmente, o conjunto de $N$ agentes e suas interconexões expressam um grafo [55, 104-106], como ilustra a Figura 3.7.
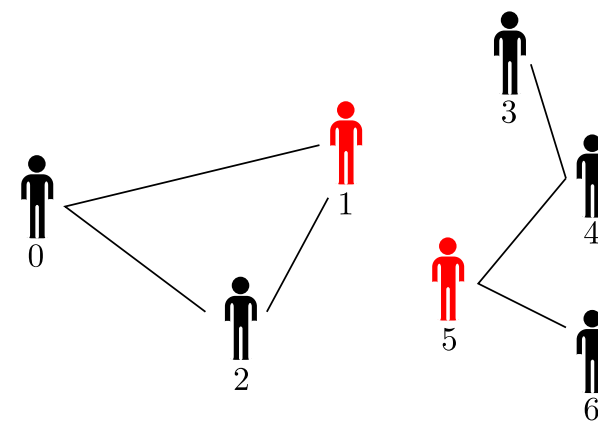

$$
A=\left(\begin{array}{lllllll} 
& 1 & 1 & & & & \\
1 & & 1 & & & & \\
1 & 1 & & & & & \\
& & & & 1 & & \\
& & & 1 & & 1 & \\
& & & & 1 & & 1
\end{array}\right)
$$

Figura 3.7: Exemplo de grafo para $N=7$ agentes. Cada agente possui uma identificação única $k=0,1, \ldots, N-1$ que independe de seu estado de saúde. A conexão entre dois agentes $k$ e $k^{\prime}$ é representada pelo segmento de reta que os une na gravura, e corresponde ao valor unitário para o elemento de matriz $A_{k^{\prime} k}$. Neste exemplo particular, observa-se que os agentes se separam em duas comunidades distintas, que não podem interagir entre si. Essa propriedade se reflete na matriz $A$ através de dois blocos disjuntos de dimensões $3 \times 3$ e $4 \times 4$, respectivamente. Isso acontece somente para enumeração conveniente dos vértices.

Por definição, grafos são estruturas matemáticas formadas por dois conjuntos, $V$ e $E$. O conjunto $V$ contém os vértices de um grafo, enquanto o $E$ contém suas arestas. Grafos são classificados de acordo com os padrões das arestas. Estes padrões podem ser oriundos de simetrias espaciais, como no caso dos cristais, ou seguir outras simetrias ou critérios. Por exemplo, um grafo completo possui todos os vértices conectados, $A_{i j}=1-\delta_{i j}$, e descreve um cenário onde todos os vértices são idênticos. Já num grafo aleatório, as $N(N-1)$ arestas ocorrem com probabilidade $p$. Naturalmente, quando $p=1$, recupera-se o grafo completo. Assim como as redes de Bravais de um sistema cristalino [51], transformações geométricas como rotação e translação também podem ser utilizadas para classificar grafos. De fato, de acordo

\footnotetext{
${ }^{10} \mathrm{~A}$ exata definição do tipo de relacionamento depende do contexto biológico, físico-químico e ambiental, por meio do qual se dá a transmissão do agente patogênico.
} 
com a definição de grafos, uma rede de Bravais nada mais é do que um grafo regular, isto é, a distribuição de arestas tem um padrão regular sob translações, rotações e/ou outras transformações espaciais.

Uma das facetas mais interessantes dessa abordagem relata que agentes reais possuem estruturas relacionais complexas [13, 23, 55, 107]. Mais ainda, tais estruturas não são completamente descorrelacionadas [55]: alguns agentes possuem maior influência do que os demais [14] ou os agentes podem apresentar o fenômeno de mundo pequeno [108]. Aspectos não-triviais de $A$, que descreve a topologia das conexões, são incorporadas em taxas de transmissão efetivas, produzindo diferentes padrões, alterando significativamente a dinâmica de propagação de doenças [21]. As propriedades que decorrem de topologias de conjuntos de grafos são objetos de estudo de teorias de redes $[55,99,104,109]$. É neste contexto que se insere a teoria de redes e suas aplicações em epidemiologia. A fim de não desviar do tema principal desta seção, discutimos maiores detalhes sobre teoria de redes no Apêndice 1, onde também fazemos a distinção entre grafos e redes. No que segue, a matriz $A$ faz referência sempre a um único grafo, enquanto $\langle A\rangle$ representa a média sobre um ensemble de grafos, os quais são representantes de uma dada rede. ${ }^{11}$

De volta ao ABEM, o passo seguinte requer a definição precisa dos possíveis estados de cada um dos $N$ agentes. No caso particular do modelo SIS, há apenas dois estados de saúde, a saber, infectado $|1\rangle_{k}$ e susceptível $|0\rangle_{k}$. O subíndice $k=$ $0,1, \ldots, N-1$, como de costume, identifica o agente enquanto 0 ou 1 , o estado de saúde. A combinação dos estados de cada agente produz uma configuração de $N$ agentes, representada pelo vetor

$$
\left|C_{\mu}\right\rangle=\left|\sigma_{0} \sigma_{1} \cdots \sigma_{N-1}\right\rangle
$$

onde

$$
\begin{aligned}
& \sigma_{k}= \begin{cases}0, & \text { se susceptível } \\
1, & \text { se infectado }\end{cases} \\
& \mu=\sigma_{0} 2^{0}+\sigma_{1} 2^{1}+\cdots+\sigma_{N-1} 2^{N-1} .
\end{aligned}
$$

\footnotetext{
${ }^{11} \mathrm{O}$ termo representante de rede remete a uma discussão muito produtiva a respeito da definição formal de redes (networks) e sua dependência com grafos. O artigo de revisão Ref. [99] estabelece logo em seus parágrafos iniciais a equivalência unívoca entre uma rede e um grafo. Entretanto, essa definição não é empregada para o caso das redes aleatórias, onde se utiliza o ensemble para caracterizar as propriedades comuns dos grafos aleatórios. Nesse caso, qualquer grafo pertencente ao ensemble é um representante da rede aleatória. A definição de redes, portanto, é imprecisa. No Apêndice 1 discutimos esta problemática em detalhes, inclusive as hipóteses que produzem as duas definições.
} 
A escolha de valores binários para $\sigma_{k}$ facilita a enumeração $\mu=0,1, \ldots, 2^{N}-1$ configurações. Outra possibilidade seria a representação em termos da projeção azimutal de spin $s=1 / 2$ [86]. Em ambos os casos, a notação remete aos conceitos e vetores discutidos no Capítulo 2. No que segue, até o final deste capítulo, reservamos os índices latinos para descrever os agentes e índices gregos para identificar configurações, isto é, $i, j, k=0,1, \ldots, N-1$ enquanto $\mu, \nu=0,1, \ldots, 2^{N}-1$.

O conjunto de $2^{N}$ vetores $\left\{\left|C_{\mu}\right\rangle\right\}$ forma uma base ortonormal e gera o espaço de Hilbert, $\mathbb{H}$ - grosso modo, isto quer dizer que existe produto escalar e norma. A ortogonalidade dos vetores de base é expressa por

$$
\left\langle C_{\mu} \mid C_{\nu}\right\rangle=\delta_{\mu \nu}
$$

Agora, muito embora $\left|C_{\mu}\right\rangle$ descreva uma configuração específica de $N$ agentes, num dado instante de tempo $t$, isso não implica que o sistema se encontre, de fato, na configuração $\left|C_{\mu}\right\rangle$. Considere o seguinte exemplo (Figura 3.8). Suponha que se observa repetidas vezes a evolução de um sistema de $N$ agentes, sempre partindo da mesma condição inicial. Decorrido o intervalo de tempo $\Delta t$, determina-se o estado de saúde de todos os agente e, portanto, identifica-se a configuração $C_{\mu}$. Digamos que, nesse exemplo, o sistema encontre-se metade das vezes na configuração $\left|C_{\nu}\right\rangle$ e a outra metade, na configuração $\left|C_{\nu^{\prime}}\right\rangle$. Assim, as configurações $\left|C_{\mu}\right\rangle$ satisfazem sua funcionalidade, que é a representação dos estados de saúde de $N$ agentes, mas a observação destas configurações possui uma probabilidade de observação. Logo, a descrição do sistema não pode ser realizada por meio de uma única configuração, mas sim pela combinação linear de configurações, cada qual com uma probabilidade de observação.

Ora, um vetor $|P(t)\rangle$ pertencente a $\mathbb{H}$ sempre pode ser escrito como a combinação linear dos elementos da base,

$$
|P(t)\rangle=\sum_{\mu} P_{\mu}(t)\left|C_{\mu}\right\rangle
$$

onde os coeficientes $P_{\mu}(t)$ exprimem as probabilidades de observação das configurações $\left|C_{\mu}\right\rangle$ correspondentes. Por carregar coeficientes que, essencialmente, representam estocasticidade, o vetor $|P(t)\rangle$ recebe a denominação de vetor probabilidade. Sua finalidade é descrever o sistema e, portanto, o elemento mais fundamental da abordagem baseada em agentes. O vínculo da probabilidade total e a conservação 


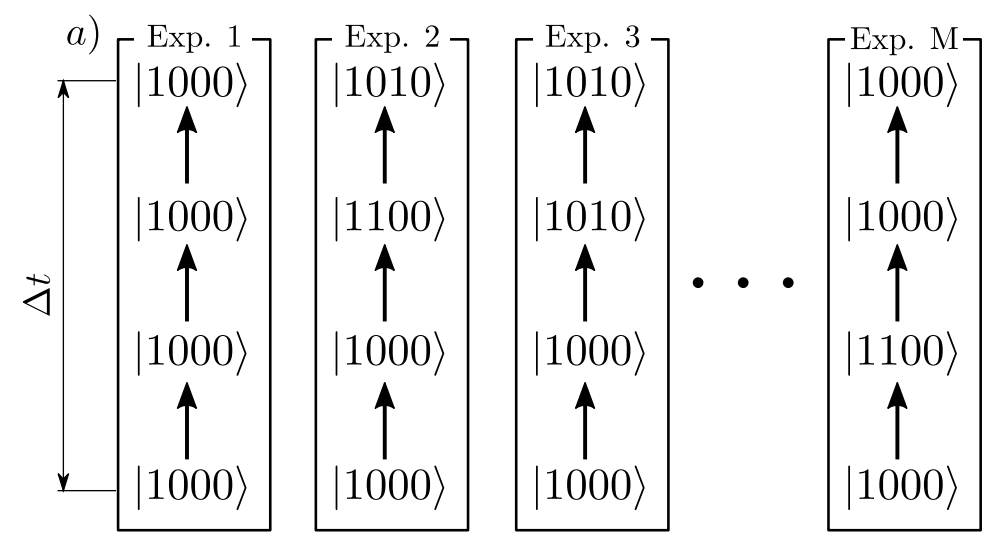

b)

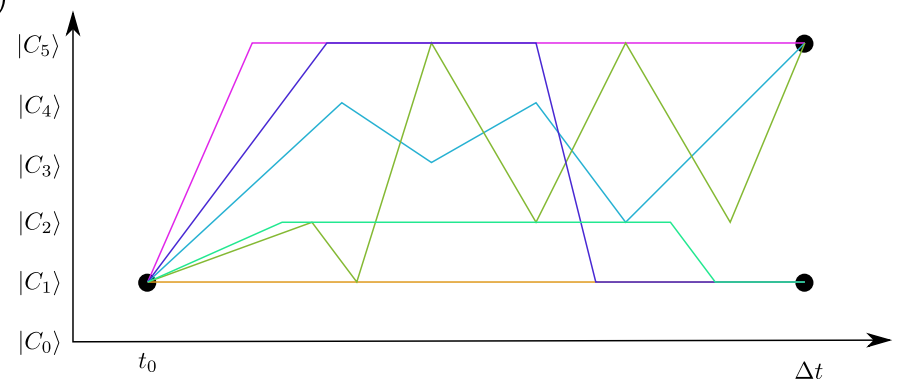

Figura 3.8: Evolução temporal de um processo estocástico. a) Um sistema de $N=4$ agentes encontra-se na configuração $\left|C_{1}\right\rangle$ como condição inicial. Decorrido um intervalo de tempo $\Delta t$, determina-se a nova configuração. Repete-se o experimento $M$ vezes e observa-se que, nesse exemplo particular, que apenas as configurações $\left|C_{1}\right\rangle$ e $\left|C_{5}\right\rangle$ são observadas como configurações finais. b) As diversas curvas de evolução experimentadas pelo sistema, partindo sempre da mesma configuração inicial. Decorrido o intervalo de tempo $\Delta t$, apenas duas configurações são observadas, com mesma frequência.

da probabilidade são expressas da seguinte maneira:

$$
\begin{aligned}
\sum_{\mu} P_{\mu}(t) & =1, \\
\frac{\partial}{\partial t} \sum_{\mu} P_{\mu}(t) & =0 .
\end{aligned}
$$

Enfatizamos que, ao contrário da Mecânica Quântica, $|P(t)\rangle$ não apresenta o fenômeno de interferência [40-42], posto que os coeficientes $P_{\mu}(t)$ já são em si probabilidades.

Antes de concluir essa seção, convém generalizar o espaço vetorial para sistemas com mais de dois estados de saúde, isto é, $q=3,4, \ldots$ que caracteriza modelos epidêmicos mais complexos como SIRS e SEIRS. Sob esta assunção, os vetores que 
representam as configurações de $N$ agentes são ${ }^{12}$

$$
\begin{aligned}
\left|C_{\mu}\right\rangle & =\left|\sigma_{0} \sigma_{1} \cdots \sigma_{N-1}\right\rangle, \\
\sigma_{k} & =0,1, \ldots, q-1, \\
\mu & =\sum_{k=0}^{N-1} \sigma_{k} q^{k} .
\end{aligned}
$$

Para ser mais preciso, utilizamos a seguinte convenção para os modelos de três estados como SIR e SIRS:

$$
\sigma_{k}= \begin{cases}0, & \text { se } R \\ 1, & \text { se } S \\ 2, & \text { se } I\end{cases}
$$

enquanto para os modelos de quatro estados como SEIR e SEIRS, temos

$$
\sigma_{k}=\left\{\begin{array}{ll}
0, & \text { se } R \\
1, & \text { se } S \\
2, & \text { se } E \\
3, & \text { se } I
\end{array} .\right.
$$

De acordo com essa convenção, os estados de saúde se tornam mais danosos com o incremento do valor de $\sigma_{k}$, refletindo a cadeia de eventos da doença, $R \rightarrow S \rightarrow$ $E \rightarrow I$. Uma representação alternativa emprega o valor $s_{k}=\sigma_{k}-s$, onde $s=1$ para SIR/SIRS e $s=3 / 2$ para SEIR/SEIRS. Essa representação faz referência aos números quânticos azimutais para spin $s=1\left(s_{k}=-1,0,1\right)$ e $s=3 / 2\left(s_{k}=\right.$ $-3 / 2,-1 / 2,1 / 2,3 / 2)$.

\subsection{Operadores e a matriz de transição}

Uma vez definido o espaço vetorial, temos sinal verde para descrever operadores. Operadores nada mais são do que as transformações entre os diferentes vetores de um espaço vetorial. Num modelo epidêmico, os processos de infecção e cura nada mais são do que transformações. Por exemplo, considere a configuração

\footnotetext{
${ }^{12} \mathrm{~A}$ enumeração de configurações utilizando a representação $q$-nária de inteiros é bastante recorrente em computação científica. Existem diversas vantagens em se utilizar essa representação, especialmente no caso dos números binários. Isto se deve à existência de operações lógicas altamente especializadas e otimizadas como AND, OR, XOR, etc. Observe que a ação destas operações sobre o número inteiro (que representa uma configuração) equivale à transformação da configuração. Embora essa técnica computacional não esteja sempre à disposição para todos os modelos, sua implementação geralmente incrementa a eficiência de códigos computacionais. A razão é que a quantidade de memória requerida para manipular os dados é menor, evitando-se buscas desnecessárias, e pelo fato de que as operações lógicas são otimizadas no pipeline dos processadores.
} 
$\left|C_{1}\right\rangle=|1000\rangle$ para $N=4 \mathrm{com} q=2$. Se por ventura o agente $k=0$ recuperar-se da infecção, então a nova configuração do sistema será $\left|C_{0}\right\rangle=|0000\rangle$. Logo, existe um operador $\hat{O}$ tal que $\hat{O}\left|C_{1}\right\rangle=\left|C_{0}\right\rangle$. Já que transformações são produto da ação de operadores e a evolução temporal nada mais é do que uma sequência sucessiva de transformações, conclui-se que a evolução temporal é um operador. Assim, o segundo passo da modelagem baseada em agentes se reduz a determinar a matriz de transição $\hat{T}$, que é o operador descritor da translação temporal por uma unidade de tempo $\delta t: 13$

$$
|P(t+\delta t)\rangle=\hat{T}|P(t)\rangle
$$

A equação acima expressa o papel de $\hat{T}$ como operador responsável pela evolução temporal do sistema. Os elementos de matriz $T_{\mu \nu}$ descrevem as probabilidades de transição que partem das configurações $\left|C_{\nu}\right\rangle$ e vão para $\left|C_{\mu}\right\rangle$. Em geral, a simetria por reversão temporal não é um requerimento intrínseco e sua ocorrência depende das regras do modelo. Quando não há tal simetria, o operador $\hat{T}$ e seu correspondente transposto $\hat{T}^{T}$ não são equivalentes. Veremos adiante que esse é exatamente o caso do modelo SIS.

Conforme discutido anteriormente, um dos elementos cruciais na formulação baseada em agentes reside no conceito de probabilidades de transição entre configurações, durante o intervalo de tempo $\delta t$. Até o presente momento, a escolha do parâmetro temporal parece arbitrária, o que não condiz com o esperado: se tomar$\operatorname{mos} \delta t=1$ ano para estudar a propagação da gripe, uma pessoa pode ser infectada e recuperar-se diversas vezes. $\mathrm{O}$ caso oposto, quando $\delta t=1$ ns também não é muito informativo pois não se espera mudanças nessa escala de tempo, ou seja, $\hat{T}$ comportar-se-ia praticamente como o operador identidade. Grosso modo, as transições tornam-se mais complexas (triviais) quanto maior (menor) o intervalo de tempo $\delta t$, como ilustra a Figura 3.9.

Para o modelo SIS/SI, as transições mais simples e não-triviais consistem na recuperação de um agente ou de um evento de transmissão de patógeno entre dois agentes distintos. As transições mais simples de um modelo são denominadas transições elementares. Assim, é razoável admitir que existe uma janela de

\footnotetext{
${ }^{13}$ Neste ponto, salienta-se que a descrição do texto difere da apresentação tradicional de processos estocásticos. Em nossa notação, $|P(t)\rangle$ é um vetor coluna em sua representação matricial. Já na Ref. [20], o vetor probabilidade é um vetor linha. Essa escolha altera a disposição dos elementos de matriz de $\hat{T}$ e leva a interpretações ligeiramente distintas quanto à evolução e regressão temporal.
} 


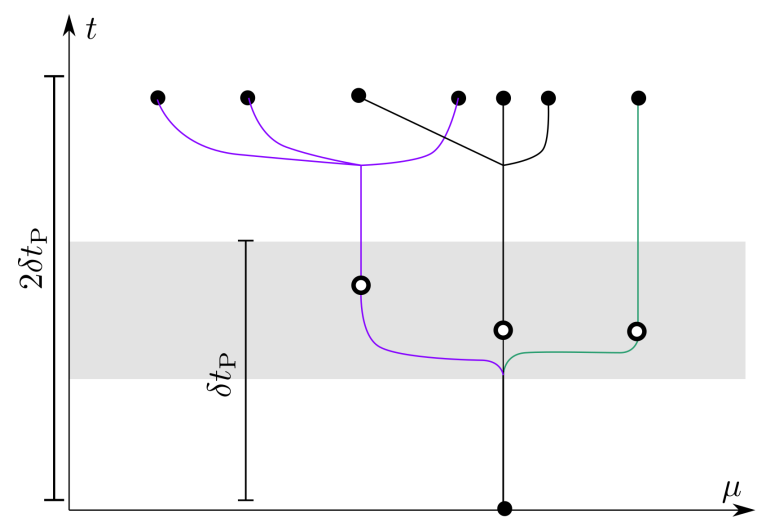

Figura 3.9: Hipótese poissônica. Transições tendem a ser mais complexas quanto maior for o parâmetro de deslocamento temporal $\delta t$. Aqui, a condição inicial é bem caracterizada por uma única configuração $\left|C_{\mu}\right\rangle$. Sua evolução temporal se fragmenta em 3 sequências intermediárias (círculos vazios), que por sua vez se fragmentam em 7 configurações (círculos cheios). A área sombreada indica a janela de intervalos de tempo $\delta t_{P}$ na qual apenas transições elementares acontecem com probabilidade significativa (3 transições). Se intervalos maiores que $\delta_{p}$ forem considerados como unidade mínima de tempo, então o número de transições por unidade de tempo também aumenta.

intervalos de tempo $\delta t$, na qual apenas uma transição elementar ocorre com probabilidade significativa, independentemente da natureza da transição elementar se cura ou transmissão. Neste caso, as probabilidades de transição entre diferentes configurações são inferidas a partir da modelagem destas como processos poissônicos independentes, que podem ser estimadas diretamente dos dados brutos - por exemplo, as taxas $\gamma$ e $\beta$. Essa inferência é a hipótese poissônica e desempenha um papel central em processos estocásticos $[21,103]$. De acordo com a hipótese de Poisson para o modelo SIS, temos $\delta t N(\beta+\gamma) \approx 1, \operatorname{logo} \delta t \approx(\beta+\gamma)^{-1} / N .{ }^{14}$ No que segue, $\delta t$ satisfaz a hipótese poissônica, o que nos permite concentrar esforços na determinação apenas de operadores que descrevem as transições elementares do modelo SIS. A composição de tais transições, conjuntamente com as taxas correspondentes, produzem a matriz de transição do modelo.

Considere o operador localizado $\hat{\sigma}_{k}^{z}$ para $q=2$. A ação deste operador sobre uma configuração de $N$ agentes resulta na própria configuração, multiplicada por +1 se o agente localizado no $k$-ésimo vértice estiver infectado ou -1 se estiver

\footnotetext{
${ }^{14}$ A dependência $N^{-1}$ para o intervalo de tempo é fato bem conhecido para aqueles versados nas artes de simulação de Monte Carlo. No jargão da área, a eterna batalha entre o "tempo de Monte Carlo" e o "tempo real". Com o incremento do número de graus de liberdade, mais eventos ocorrem por passo da simulação. Para compensar, a unidade de tempo é reescalada, compatibilizando a escala de tempo entre sistemas com diferente número de graus de liberdade. Nessa discussão desconsideramos o expoente crítico dinâmico e outros efeitos coletivos.
} 
susceptível, isto é,

$$
\hat{\sigma}_{k}^{z}\left|\sigma_{0} \sigma_{1} \cdots \sigma_{k} \cdots \sigma_{N-1}\right\rangle=\left(2 \sigma_{k}-1\right)\left|\sigma_{0} \sigma_{1} \cdots \sigma_{k} \cdots \sigma_{N-1}\right\rangle
$$

Apesar de trivial, também convém definir o operador número localizado, $\hat{n}_{k}=\left(\hat{\sigma}_{k}^{z}+\right.$ 1) $/ 2$, que extrai o estado do agente no vértice $k$ :

$$
\hat{n}_{k}\left|\sigma_{0} \sigma_{1} \cdots \sigma_{k} \cdots \sigma_{N-1}\right\rangle=\sigma_{k}\left|\sigma_{0} \sigma_{1} \cdots \sigma_{k} \cdots \sigma_{N-1}\right\rangle .
$$

Assim, o número total de agentes infectados em uma dada configuração é determinado pelo operador

$$
\hat{n}=\sum_{k} \hat{n}_{k}
$$

Por definição, as configurações $\left|C_{\mu}\right\rangle$ são autovetores dos operadores localiza$\operatorname{dos} \hat{\sigma}_{k}^{z}$ e $\hat{n}_{k}$. Consequentemente, estes operadores localizados não executam transições entre diferentes configurações, ou seja, não descrevem as eventos de transmissão ou cura dos agentes. Para remediar esse problema, introduz-se dois novos operadores localizados, em sintonia com o conteúdo discutido no Capítulo 2:

$$
\begin{aligned}
& \hat{\sigma}_{k}^{+}\left|\sigma_{0} \cdots 0_{k} \cdots \sigma_{N-1}\right\rangle=\left|\sigma_{0} \cdots 1_{k} \cdots \sigma_{N-1}\right\rangle, \\
& \hat{\sigma}_{k}^{-}\left|\sigma_{0} \cdots 1_{k} \cdots \sigma_{N-1}\right\rangle=\left|\sigma_{0} \cdots 0_{k} \cdots \sigma_{N-1}\right\rangle,
\end{aligned}
$$

nulo para qualquer outro caso. Aqui, o operador localizado $\hat{\sigma}_{k}^{+}$muda o estado de saúde do agente no $k$-ésimo vértice de susceptível para infectado, enquanto o operador $\hat{\sigma}_{k}^{+}$produz efeito inverso. A trinca de operadores localizados $\hat{\sigma}_{k}^{z, \pm}$ satisfazem as propriedades algébricas locais

$$
\begin{aligned}
& {\left[\hat{\sigma}_{k}^{z}, \hat{\sigma}_{k}^{ \pm}\right]= \pm 2 \hat{\sigma}_{k}^{ \pm}} \\
& {\left[\hat{\sigma}_{k}^{+}, \hat{\sigma}_{k}^{-}\right]=\sigma_{k}^{z},}
\end{aligned}
$$

que corresponde às constantes de estrutura da álgebra su(2). Já em relação às relações de comutação e anticomutação de agentes distintos resulta em

$$
\begin{aligned}
{\left[\hat{\sigma}_{k}^{a}, \hat{\sigma}_{k^{\prime}}^{b}\right] } & =0, \\
\left\{\hat{\sigma}_{k}^{+}, \hat{\sigma}_{k}^{-}\right\} & =1,
\end{aligned}
$$

para $k \neq k^{\prime}$ e $a, b=z, \pm$. Assim como ocorre em sistemas fortemente correlacionados e sistemas de spin, os operadores em vértices distintos comutam, enquanto operadores no mesmo vértice anticomutam [10]. Dependendo da metodologia adotada para 
se resolver o problema, pode ser necessário selecionar uma única natureza para os operadores: ou completamente fermiônicos ou completamente bosônicos. O motivo é que a identificação de quasipartículas se dá somente a partir de tal escolha, com grande influência no preenchimento de níveis, densidade de ocupação e distribuição de probabilidade $[10,17,110]$. É possível evitar esse problema e trabalhar diretamente com o momento angular de muitos corpos $\hat{J}$, especialmente quando impostas aproximações de campo médio ou se o processo se dá em grafos isotrópicos.

No modelo SIS, um agente infectado no vértice $k$ é sujeito a três distintas possibilidades elementares frente o passar do tempo, a saber, curar-se, infectar um de seus vizinhos susceptíveis ou não fazer nada. Essas três mudanças correspondem a três operadores distintos, como mostramos a seguir. O operador $\hat{\sigma}_{k}^{-} \hat{n}_{k}$ equivale à ação de cura do $k$-ésimo agente: da direita para esquerda, o operador número checa se há um agente infectado no $k$-ésimo vértice; em caso positivo o operador $\hat{\sigma}_{k}^{-}$ inverte o estado de saúde do agente, ou produz o vetor nulo caso contrário. ${ }^{15}$ Como aplicação, considere o vetor configuração $\left|C_{5}\right\rangle$ para um sistema com $N=4$ agente, tal que

$$
\left(\sum_{k} \hat{\sigma}_{k}^{-} \hat{n}_{k}\right)\left|C_{5}\right\rangle=\left|C_{1}\right\rangle+\left|C_{4}\right\rangle,
$$

ou na notação binária,

$$
\left(\sum_{k} \hat{\sigma}_{k}^{-} \hat{n}_{k}\right)|1010\rangle=|1000\rangle+|0010\rangle .
$$

Já o operador $A_{j k} \hat{\sigma}_{j}^{+} \hat{n}_{k}$ expressa a transmissão da doença para o $j$-ésimo a partir do $k$-ésimo agente, caso este encontre-se previamente infectado. Aqui, o valor binário do elemento da matriz de adjacência corrige a ação dos operadores localizados dependendo da existência ou não da conexão entre os agentes em questão. Para exemplificar este termo, considere a matriz seguinte matriz de adjacência:

$$
A=\left(\begin{array}{llll}
0 & 1 & 0 & 1 \\
1 & 0 & 1 & 0 \\
0 & 1 & 0 & 1 \\
1 & 0 & 1 & 0
\end{array}\right)
$$

O grafo correspondente a essa matriz de adjacência consiste em agentes dispostos em um círculo, onde cada agente conecta-se somente com o vizinho mais próximo.

\footnotetext{
${ }^{15}$ Existe uma certa redundância aqui, pois $\hat{\sigma}_{k}^{-} \equiv \hat{\sigma}_{k}^{-} \hat{n}_{k}=\hat{\sigma}_{k}^{-}\left(\hat{n}_{k}\right)^{l}$ para qualquer $l$ inteiro positivo, devido aos autovalores $n_{k}=0,1$.
} 
Assim,

$$
\left(\sum_{j k} A_{j k} \hat{\sigma}_{j}^{+} \hat{n}_{k}\right)\left|C_{5}\right\rangle=2\left|C_{7}\right\rangle+2\left|C_{13}\right\rangle .
$$

Das três transições possíveis no modelo SIS, resta somente o evento no qual o vetor permanece inalterado. Para este caso, existem três operadores relevantes: o operador identidade $\mathbb{1}$, o operador número $\hat{n}_{k}$ e o operador $\left(1-\hat{n}_{j}\right) \hat{n_{k}}$. A ideia por trás desta seleção de objetos é contabilizar o decréscimo da probabilidade de permanecer inalterado devido às transições entre configurações distintas. Por exemplo, $\sum_{k} \hat{n}_{k}\left|C_{\mu}\right\rangle=n_{\mu}\left|C_{\mu}\right\rangle$ diz que a configuração $\left|C_{\mu}\right\rangle$ possui $n_{\mu}$ agentes infectados, os quais podem eventualmente se curar. Portanto, probabilidade de não ocorrer transição deve levar em conta o fator $-\gamma \delta t n_{\mu}$ para a configuração $\left|C_{\mu}\right\rangle$. O mesmo segue para o operador $\left(1-\hat{n}_{j}\right) \hat{n}_{k}$ em relação à transmissão da doença.

De posse dos operadores correspondentes às três transições elementares do modelo SIS, e conjunto com as respectivas taxas de transmissão por agente $\beta / N$ e de cura $\gamma$, a matriz de transição fica

$$
\hat{T}=\mathbb{1}-\frac{\beta \delta t}{N} \sum_{k j}\left[A_{j k}\left(1-\hat{n}_{j}-\hat{\sigma}_{j}^{+}\right)+\Gamma \delta_{k j}\left(1-\hat{\sigma}_{j}^{-}\right)\right] \hat{n}_{k},
$$

onde o acoplamento $\Gamma=\gamma N / \beta$ e $\delta_{k j}$ é o delta de Kronecker. A matriz de transmissão $\hat{T}$ na Eq. (3.32) contempla a heterogeneidade populacional, por meio da matriz de adjacência $A$, nos eventos de transmissão. Assim, a abordagem baseada em agentes é capaz de mimicar os efeitos amplificados daqueles entes da população que possuem vasta rede de contatos, por exemplo. A Figura 3.10 mostra a ação de $\hat{T}$ sobre uma configuração de agentes, organizados como grafo regular periódico $\left(A_{j, k}=\delta_{k, j \pm 1}\right)$.

Uma forma de se obter as probabilidades $P_{\mu}(t)$ a partir de $\hat{T}$ é através da iteração sucessiva da Eq. (3.21), a partir de uma condição inicial $|P(0)\rangle$. A Figura 3.11 exibe a evolução das probabilidades $P_{\mu}(t)$ para $\mu=0,5,2^{N}-1$, para um grafo completo $A_{i j}=1-\delta_{i j}$ para diversos valores de $\Gamma$. A condição inicial descreve apenas um agente infectado no vértice $k=0$, isto é, $P_{1}(0)=1$.

A inspeção da Eq. (3.32) mostra que $\hat{T}$ não é Hermitiano. A principal consequência disto se desmembra na existência de dois conjuntos de autovetores, os autovetores a esquerda $\left\{\left\langle\chi_{\mu}\right|\right\}$ e a direita $\left\{\left|\phi_{\mu}\right\rangle\right\}$, para um mesmo conjunto de autovalores. Mais ainda, tais autovetores não se relacionam pela conjugação Hermitiana, como geralmente ocorre para operadores simétricos. Dado que as dimensões 


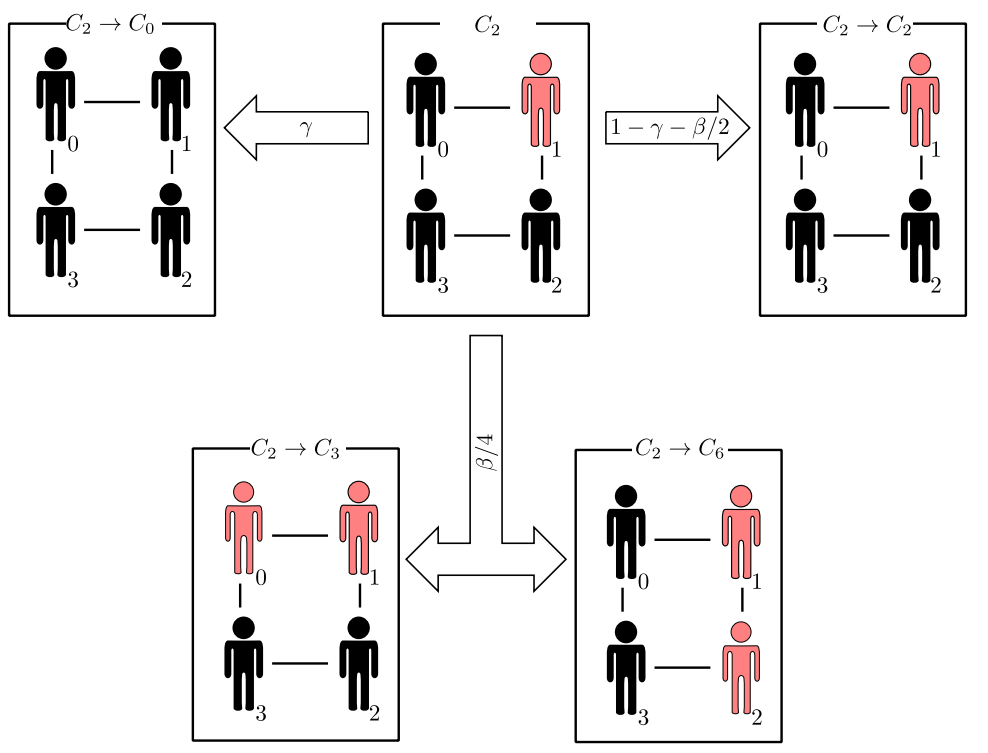

Figura 3.10: Exemplo de processo epidêmico SIS no grafo regular periódico com $N=4$. Neste grafo regular, cada agente possui exatamente duas conexões com dois agentes distintos, formando uma estrutura cíclica de período $N$. Aqui, a matriz de transmissão atua sobre a configuração $\left|C_{2}\right\rangle=|0100\rangle$. Esta configuração resulta em quatro possíveis configurações após o intervalo de tempo $\delta t$. O agente em $k=1$ pode se curar com probabilidade $\gamma$, produzindo a configuração $\left|C_{0}\right\rangle$. O agente em $k=1$ também pode transmitir o patógeno ou para o agente em $k=0$ ou $k=2$, cada evento com probabilidade $\beta / N$. Por fim, o agente pode simplesmente não fazer nada, permanecendo na configuração $\left|C_{2}\right\rangle$ com probabilidade $1-\gamma-2 \beta / N$.

de $\hat{T}$ são $2^{N} \times 2^{N}$, o cálculo de dois conjuntos de autovetores torna uma tarefa difícil em excruciante, pois além disso a decomposição da identidade (completeza), $\mathbb{1}=\sum_{\mu}\left|\phi_{\mu}\right\rangle\left\langle\chi_{\mu}\right|$, ainda deve ser satisfeita mesmo que a norma de tais vetores não seja unitária. Em termos computacionais, esse problema muitas vezes não converge corretamente para a solução correta. Esses características são críticas frequentes à abordagem baseada em agentes, pois restringe significativamente o tamanho da população a ser estudada, e geralmente se limita à análise computacional ou numérica. Veremos na Seção 3.6 como contornar esse problema, especialmente para a análise dos estados de equilíbrio.

Convém salientar que a complexidade introduzida pela topologia de conexões dos grafos é removida ao se considerar a aproximação de campo médio. Nessa aproximação, todos os agentes são equivalentes e, por isso, todos são conectados, ou seja, assume-se $A_{i j}=\left(1-\delta_{i j}\right)$, que nada mais é do que o grafo completo [106]. Observe que essa aproximação também é compatível com a abordagem compartimental. De fato, esse é um dos requerimentos necessários para se recuperar a Eq. (3.5), como 

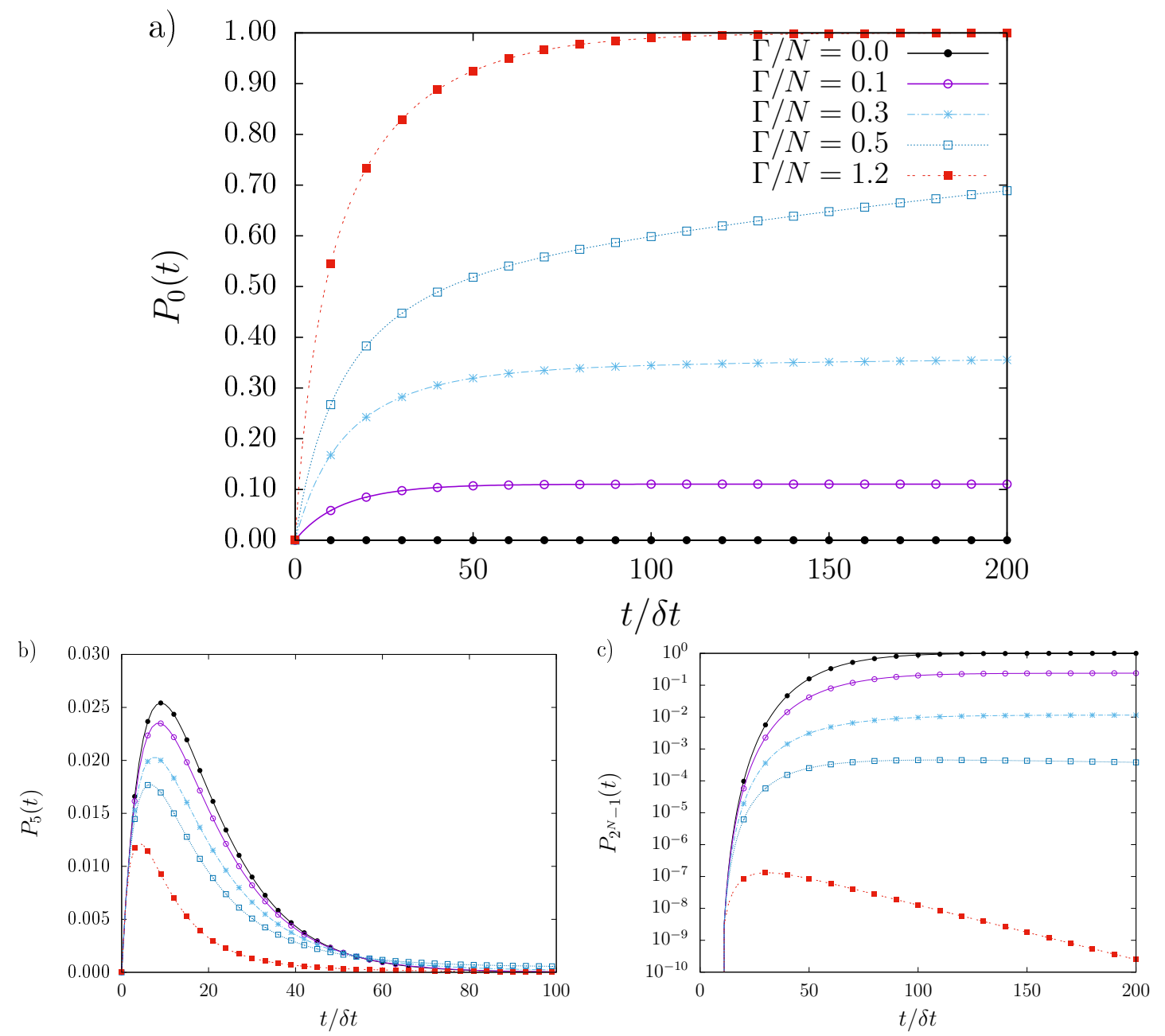

Figura 3.11: Evolução temporal das probabilidades das configurações $\left|C_{\mu}\right\rangle\left(\mu=0,5\right.$ e $\left.2^{N}-1\right)$, com $N=12$ num grafo completo. Mostra-se apenas as primeiras 200 iterações temporais. Em a), a probabilidade $P_{0}(t)$ de se observar todos os agentes no estado susceptível para diversos valores de $\Gamma$. Note que para $\Gamma=0$ (círculos pretos preenchidos), recupera-se o modelo SI, que não contém eventos de cura. b) $\mathrm{O}$ estado intermediário com dois agentes infectados, $k=0$ e $k=2$, é efêmero e possui uma duração característica de acordo com o acoplamento $\Gamma$. c) A probabilidade da configuração com todos os agentes infectados, $P_{2^{N}-1}(t)$, na escala log. Evidencia-se o decaimento exponencial para $\Gamma / N=1.2$. Para os acoplamentos intermediários $\Gamma / N=0.3$ e 0.5 , a configuração de equilíbrio $\left|C_{0}\right\rangle$ é atingida após um longo intervalo de tempo (transiente). Imagem reproduzida da Ref. [86], sob permissão acadêmica.

veremos mais adiante. Por fim, a abordagem operatorial é facilmente modificada a fim de contemplar dependência temporal seja por meio da matriz de adjacência $A \equiv A(t)$ ou das taxas de infecção e cura, $\beta \equiv \beta(t)$ e $\gamma \equiv \gamma(t) .{ }^{16}$ Ademais, discutimos propriedades oriundas de simetrias finitas de $\hat{T}$ no Apêndice 2. Apesar de não

\footnotetext{
${ }^{16} \mathrm{O}$ estudo da teoria de perturbação dependente do tempo para as taxas epidemiológicas e de $A$ são excelentes vias de pesquisa, com ampla aplicabilidade em cenários reais. Em particular, permite a dedução dos efeitos causados por ações governamentais ou de órgãos responsáveis no combate às epidemias. Atualmente, o método para se avaliar tais efeitos consiste na execução sucessiva de simulações de Monte Carlo.
} 
ser necessário para o entendimento imediato do que segue, recomendamos sua leitura tanto pela questão computacional quanto pelos conceitos de setores e quantidades conservadas, tópicos geralmente muito especializados em Física.

No Apêndice 3 ampliamos a discussão sobre a construção da matriz de transição para sistemas epidêmicos mais complexos, como os modelos SIRS e SEIRS. Por completeza, também abordamos a difusão do patógeno e modelagem de endemias.

\subsection{Observáveis e evolução temporal}

O lado esquerda da Eq. (3.21) pode ser expandido numa série de potência em $\delta t$

$$
|P(t)\rangle+\left(\delta t \frac{d}{d t}\right)|P(t)\rangle+o\left(\delta t^{2}\right)=\hat{T}|P(t)\rangle .
$$

Desprezando-se os termos de ordem $o\left(\delta t^{2}\right)$, a expressão acima torna-se um sistema de equações diferenciais:

$$
\frac{d P_{\mu}}{d t}=-\sum_{\nu} H_{\mu \nu} P_{\nu}(t)
$$

onde $\hat{H} \equiv(\mathbb{1}-\hat{T}) / \delta t$ é o gerador da translação temporal do sistema, ou seja, rege integralmente o processo Markoviano. ${ }^{17}$ Esse sistema de equações diferenciais é muito semelhante aqueles da Mecânica Quântica. Aliás, essa é a justificativa para a escolha da nomenclatura de $\hat{H}$, pois desempenha papel análogo ao do Hamiltoniano da teoria quântica, regendo a dinâmica por meio de seus autovalores e autovetores.

Formalmente, a Eq. (3.34) possui a seguinte solução:

$$
|P(t)\rangle=\mathrm{e}^{-\hat{H} t}|P(0)\rangle,
$$

uma vez que $\hat{H}$ independe do tempo. Agora, para que essa solução faça sentido, precisa-se lançar mão da decomposição espectral de $\hat{H}$. Lembrando que $\hat{T}$ não é necessariamente Hermitiano, embora composto somente de elementos reais e positivos na base $\left\{\left|C_{\mu}\right\rangle\right\}$, conclui-se que $\hat{H}$ possui dois autovetores $\left\langle\chi_{\mu}\right|$ e $\left|\phi_{\mu}\right\rangle$ para cada autovalor $\lambda_{\mu}$, os quais não necessariamente se relacionam pela conjugação hermitiana. O espectro de autovalores é positivo $\lambda_{\mu} \geqslant 0$ para qualquer

\footnotetext{
${ }^{17}$ Grosso modo, a Eq. (3.34) nada mais é do que uma roupagem operatorial para a equação de FokkerPlanck [20]. Também é conhecida por equação de Kolmogorov. No caso do movimento browniano, a representação espacial do operador $\hat{H}=-D \partial_{x}^{2}$ em uma dimensão produz uma função densidade probabilidade $P(x, t)=(4 \pi D t)^{-1 / 2} \exp \left(-x^{2} / 4 D t\right)$, onde $D=T v$ é a constante de difusão, $v$ é a mobilidade e $T$, a temperatura (relação de Einstein). Neste caso, a resolução da equação de Fokker-Planck pode ser obtida mediante transformada de Fourier para a componente espacial.
} 
$\mu=0,1, \ldots, 2^{N}-1$. Além disso, os autovetores sempre satisfazem a decomposição da identidade ${ }^{18} \sum_{\mu}\left|\phi_{\mu}\right\rangle\left\langle\chi_{\mu}\right|=\mathbb{1}$. Como usual, essa propriedade pode ser inserida na Eq. (3.34), produzindo o resultado

$$
|P(t)\rangle=\sum_{\mu} \pi_{\mu} \mathrm{e}^{-\lambda_{\mu} t}\left|\phi_{\mu}\right\rangle
$$

com $\pi_{\mu}=\left\langle\chi_{\mu} \mid P(0)\right\rangle$. Duas lições podem ser aprendidas aqui. Primeiro, como os autovalores nunca são negativos, apenas os autoestados com autovalores nulos $\lambda_{\mu}=0$ sobrevivem para $\lambda_{\mu} t \gg 1$. Isso quer dizer que tais autoestados são estados estacionários. Segundo, a combinação de estados estacionários que caracteriza o regime de equilíbrio, exige o conhecimento do produto escalar entre $\left\langle\chi_{\mu}\right|$ e o estado inicial $|P(0)\rangle$. Portanto, faz-se mister calcular tanto os vetores à esquerda quanto os vetores à direita.

Seja o vetor $|\Phi\rangle=\sum_{\mu}\left|C_{\mu}\right\rangle$ tal que $|\Phi|^{2}=2^{N}$. O produto escalar $\langle\Phi \mid P(t)\rangle=$ $\sum_{\mu} P_{\mu}(t)=1$ para qualquer instante de tempo $t$ e expressa a conservação da probabilidade total. A derivada temporal do produto escalar da Eq. (3.36) por $|\Phi\rangle$ produz

$$
\begin{aligned}
r_{\nu \mu} & =\left\langle C_{\nu} \mid \phi_{\mu}\right\rangle\left\langle\chi_{\mu} \mid P(0)\right\rangle, \\
0 & =\sum_{\mu \nu} r_{\nu \mu} \lambda_{\mu} \mathrm{e}^{-\lambda_{\mu} t}
\end{aligned}
$$

válida para qualquer instante de tempo. Note que exponenciais tornam-se nulas no equilíbrio para estados transientes, enquanto a contribuição de estados estacionários anula-se devido ao termo linear $\lambda_{\mu}$. Porém, para $t=0$, novamente não há contribuição dos estados estacionários mas os estados transientes satisfazem

$$
\sum_{\mu}^{\prime} \lambda_{\mu} \pi_{\mu}\left\langle\Phi \mid \phi_{\mu}\right\rangle=0
$$

onde a soma corre somente sobre estados transientes. Essa equação introduz um vínculo mas, na prática, possui serventia puramente computacional, para verificar a conservação da probabilidade.

Introduzimos uma nova ferramenta no nosso maquinário com o operador $\hat{\Phi}=|\Phi\rangle\langle\Phi|$. Esse operador possui a seguinte propriedade $\hat{\Phi}^{m}=2^{N(m-1)} \hat{\Phi}$. Se $\omega_{\nu}$

\footnotetext{
${ }^{18} \mathrm{~A}$ prova mais simples e breve para se demonstrar esse teorema se encontra na Ref. [111]. Para simples conferência, considere a matriz $(2 \times 2) \hat{T}=\mathbb{1}-\alpha \hat{n}+\alpha \hat{\sigma}^{+}$, cujos autovalores são $\lambda_{0}=1$ e $\lambda_{1}=(1-\alpha)$. Os autovetores à direita são $\left|\phi_{0}\right\rangle=|0\rangle$ e $\left|\phi_{1}\right\rangle=|1\rangle-|0\rangle$. Já os autovalores à esquerda são $\left\langle\chi_{0}\right|=\langle 0|+\langle 1|$ e $\left\langle\chi_{1}\right|=\langle 1|$. Esses vetores satisfazem as relações $\left\langle\chi_{\mu} \mid \phi_{\nu}\right\rangle=\delta_{\mu \nu}$ e $\sum_{\mu=0}^{1}\left|\phi_{\mu}\right\rangle\left\langle\chi_{\mu}\right|=\mathbb{1}$, muito embora não tenham norma unitária.
} 
é um autovalor de $\hat{\Phi}$, então $\left(2^{-N} \omega_{\nu}\right)^{m}=2^{-N} \omega_{\nu}$ para qualquer $m$ inteiro positivo, logo os autovalores são multiplamente degenerados e valem $\omega_{\nu}=0$, exceto pelo autovalor $\omega_{0}=2^{N}$, cujo autovetor é $|\Phi\rangle$. Por completeza, uma escolha para os outros autovetores é dada por $\left(\left|C_{\nu}\right\rangle-\left|C_{0}\right\rangle\right) / \sqrt{2}$ com autovalor $\omega_{\nu}=0$.

Considere agora o valor esperado $\langle P(t)|\hat{\Phi}| P(t)\rangle=1$, que também descreve o vínculo da conservação da probabilidade. Podemos complicar um pouco mais e considerar o número médio de infectados

$$
\langle n(t)\rangle \equiv\langle P(t)|\hat{\Phi} \hat{n}| P(t)\rangle=\sum_{\mu} n_{\mu} P_{\mu}(t) .
$$

Aqui, utilizamos a notação $n_{\mu}=\left\langle\Phi\left|\sum_{k} \hat{n}_{k}\right| C_{\mu}\right\rangle$ para expressar o número de infectados na configuração $C_{\mu}$. Analogamente, o número quadrático médio de infectados é

$$
\left\langle n^{2}(t)\right\rangle \equiv\left\langle P(t)\left|\hat{\Phi} \hat{n}^{2}\right| P(t)\right\rangle=\sum_{\mu} n_{\mu}^{2} P_{\mu}(t),
$$

com variância $\sigma^{2}=\left\langle n^{2}\right\rangle-\langle n\rangle^{2}$. As variáveis descritivas $\langle n\rangle,\left\langle n^{2}\right\rangle$ e $\sigma^{2}$ são de grande interesse, e mais utilizadas, nos estudos epidemiológicos. Em particular, quando a variância tende a zero, a média é um excelente estimador para a população e recupera-se a abordagem compartimental. ${ }^{19}$ A Figura 3.12 ilustra a evolução temporal da média e do desvio padrão para diferentes valores de $\Gamma$, enfatizando a diferença entre os valores esperados na abordagem compartimental e na abordagem baseada em agentes. Mais especificamente, valores não-nulos para o desvio padrão e decaimentos do tipo lei de potência mesmo para valores de $\Gamma / N \neq 1$.

Em princípio, as estatísticas também possuem decomposições espectral:

$$
\begin{aligned}
\langle n(t)\rangle & =\sum_{\mu \nu} n_{\mu} r_{\mu \nu} \mathrm{e}^{-\lambda_{\nu} t} \\
\sigma^{2}(t) & =\sum_{\mu \nu} n_{\mu}^{2} r_{\mu \nu} \mathrm{e}^{-\lambda_{\nu} t}-\langle n(t)\rangle^{2}, \\
r_{\mu \nu} & =\left\langle C_{\mu} \mid \phi_{\nu}\right\rangle\left\langle\chi_{\nu} \mid P(0)\right\rangle .
\end{aligned}
$$

No equilíbrio, apenas os estados estacionários sobrevivem. Assim, os coeficientes $\sum_{\nu}^{\prime} r_{\mu \nu}=\tilde{r}_{\mu}$ ponderam o número de infectados provenientes de cada configuração. Se o regime de equilíbrio for composto por apenas um estado, então $\tilde{r}$ é constante

\footnotetext{
${ }^{19} \mathrm{Na}$ aproximação de campo médio, a variável relevante do problema é muito bem descrita por seu valor médio. Na Eq. (3.40), outro formato da aproximação de campo médio estabelece que $\sum_{\mu} n_{\mu}^{2} P_{\mu}(t) \approx \sum_{\mu} n_{\mu}\langle n\rangle P_{\mu}(t)=\langle n\rangle^{2}$. Como resultado, $\sigma^{2}=0$ nesta aproximação.
} 

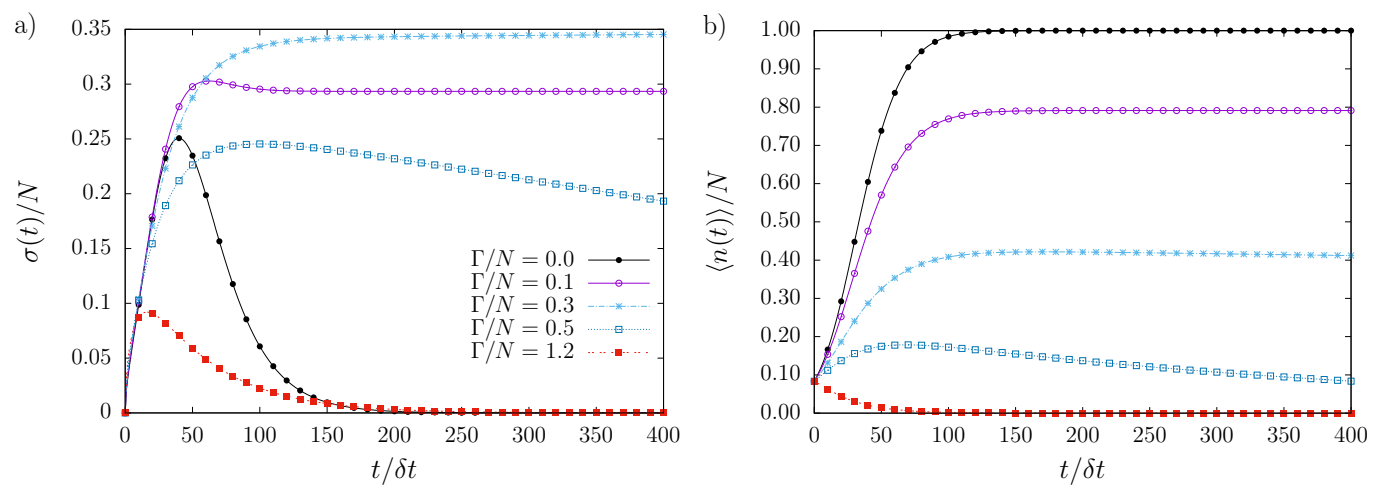

Figura 3.12: Desvio padrão $\sigma(t)$ e número médio de infectados $\langle n(t)\rangle$ para o modelo SIS com $N=12$. Os agentes desta população finita se conectam de acordo com um grafo completo, a fim de maximizar a semelhança com as hipóteses da abordagem compartimental. a) Independente do valor do acoplamento $\Gamma$, o desvio padrão assume valor não-nulo em algum momento ao longo de sua evolução temporal. À exceção dos casos limites, onde toda população torna-se infectada ou susceptível, a desvio-padrão assume valores diferentes de zero mesmo durante o equilíbrio para $0<\langle n\rangle / N<1$, contrariando a hipótese da abordagem compartimental. Também se nota um decaimento tipo lei de potência para $\Gamma / N=0.5$ b) $\mathrm{O}$ valor intermediário $\Gamma / N=0.5$ decai lentamente como uma lei de potência mais suave que o desvio.

e a variância se anula. Se a composição do equilíbrio for mais complexa, então $\sigma^{2}=\sum_{\mu}^{\prime} \tilde{r}_{\mu} n_{\mu}\left(n_{\mu}-\langle n\rangle\right)$. Sob esta circunstância, $\sigma^{2}$ se anula quando o número de infectados das configurações dos estados estacionários - que compõem o regime de equilíbrio - são iguais à própria média. Em outras palavras, se o número de infectados for uma quantidade conservada, no equilíbrio, então $\sigma^{2}=0$, mesmo que as identidades dos infectados mudem ao longo do tempo. Uma analogia adequada seria um copo, contendo um volume de água fixo, mas sujeito a movimentos: o formato da água muda, mas não o seu volume.

Ao longo da discussão destas duas últimas seções, apresentamos evidências que sugerem que é possível deduzir a Eq. (3.5) a partir da Eq. (3.34). No que segue, demonstramos essa afirmativa, enfatizando os requerimentos e aproximações. P

Os produtos escalares de $\langle\Phi| \hat{n}$ e de $\langle\Phi| \hat{n}$ com a Eq. (3.34) produzem, respectivamente,

$$
\begin{aligned}
-\frac{d}{d t}\langle n\rangle= & +\frac{\beta}{N} \sum_{\mu \nu} \sum_{i j k} A_{i j} P_{\nu}\left\langle C_{\mu}\left|\hat{n}_{k}\left(1-\hat{n}_{i}\right) \hat{n}_{j}-\hat{n}_{k} \hat{\sigma}_{i}^{+} \hat{n}_{j}\right| C_{\nu}\right\rangle+ \\
& +\gamma \sum_{\mu \nu} \sum_{i k} P_{\nu}\left\langle C_{\mu}\left|\hat{n}_{k}\left(\hat{n}_{i}-\hat{\sigma}_{i}^{-}\right)\right| C_{\nu}\right\rangle, \\
-\frac{d}{d t}\left\langle n^{2}\right\rangle= & +\frac{\beta}{N} \sum_{\mu \nu} \sum_{i j k l} A_{i j} P_{\nu}\left\langle C_{\mu}\left|\hat{n}_{l} \hat{n}_{k}\left(1-\hat{n}_{i}\right) \hat{n}_{j}-\hat{n}_{l} \hat{n}_{k} \hat{\sigma}_{i}^{+} \hat{n}_{j}\right| C_{\nu}\right\rangle+
\end{aligned}
$$




$$
+\gamma \sum_{\mu \nu} \sum_{i k l} P_{\nu}\left\langle C_{\mu}\left|\hat{n}_{l} \hat{n}_{k}\left(\hat{n}_{i}-\hat{\sigma}_{i}^{-}\right)\right| C_{\nu}\right\rangle
$$

Para uma população extremamente homogênea, todos os agentes devem ser equivalentes. Essa assunção se traduz por meio da adoção de um grafo completo para descrever a rede de contatos dos agentes. Para o grafo completo, $A_{i j}=1-\delta_{i j}$, de maneira que podemos calcular facilmente os seguintes valores esperados:

$$
\begin{aligned}
\left\langle C_{\mu}\left|\sum_{k=1}^{N} \hat{\sigma}_{k}^{+}\right| C_{\nu}\right\rangle & =\delta_{n_{\mu}, n_{\nu}+1}\left(N-n_{\nu}\right), \\
\left\langle C_{\mu}\left|\sum_{k=1}^{N} \hat{\sigma}_{k}^{-}\right| C_{\nu}\right\rangle & =\delta_{n_{\mu}, n_{\nu}-1} n_{\nu},
\end{aligned}
$$

Note que $\delta_{n_{\mu}, n_{\nu} \pm 1}$ garante o aumento ou diminuição do número de infectados, enquanto $N-n_{\nu}\left(n_{\nu}\right)$ é o número de agentes susceptíveis (infectados), portanto, passíveis de mudar de estado. A substituição das Eqs. (3.43a) e (3.43b) nas Eq. (3.42a) e (3.42b), seguido da álgebra elementar, produz

$$
\begin{aligned}
\frac{d}{d t}\langle n\rangle & =(\beta-\gamma)\langle n\rangle-\frac{\beta}{N}\left\langle n^{2}\right\rangle, \\
\frac{1}{2} \frac{d}{d t}\left\langle n^{2}\right\rangle & =(\beta-\gamma)\left(\left\langle n^{2}\right\rangle-\frac{\langle n\rangle^{2}}{2}\right)-\frac{\beta}{N}\left(\left\langle n^{3}\right\rangle-\frac{\left\langle n^{2}\right\rangle\langle n\rangle}{2}\right)+\gamma\langle n\rangle .
\end{aligned}
$$

Essas equações são exatas para o grafo completo. Note que a Eq. (3.44a) carrega $\left\langle n^{2}\right\rangle$, enquanto a Eq. (3.44b) requer o conhecimento de $\left\langle n^{3}\right\rangle$. Se considerarmos outras momentos estatísticos, a recorrência com a ordem subsequente persiste.

Convém analisar a Eq. (3.44a) no equilíbrio. Um cenário muito estudado 20 neste limite é o caso estacionário $(d / d t)\langle n\rangle=0$, que por sua vez resulta na igualdade $\left\langle n^{2}\right\rangle=(1-\gamma / \beta) N\langle n\rangle$ constante. Mais ainda, a variância $\sigma^{2}=\left\langle n^{2}\right\rangle-\langle n\rangle^{2}$ anula-se no equilíbrio para o caso $\langle n\rangle=N(1-\gamma / \beta)$. Note que essa conclusão é produto da assunção que o número de infectados é constante no equilíbrio. Ocorre que essa assunção no equilíbrio produz o resultado da aproximação de campo médio, $\left\langle n^{2}\right\rangle \approx\langle n\rangle^{2}$ para $t$ arbitrário. Logo, os resultados de campo médio são ótimos para o grafo completo.

Desprezando-se flutuações no número de agentes infectados, $\sum_{\mu} P_{\mu} n_{\mu}^{2} \approx\langle n\rangle^{2}$, torna-se possível derivar a equação compartimental:

$$
\frac{d \rho}{d t}=\beta \rho(1-\rho)-\gamma \rho
$$

\footnotetext{
${ }^{20}$ Outro cenário muito plausível, e mais apropriado para populações finitas, determina que a quantidade conservada é a variância, com dependência inversamente proporcional ao tamanho da população.
} 
onde novamente $\rho=\langle n\rangle / N$. Apesar dessa dedução ser realizada no contexto de grafos completos, esse raciocínio pode ser estendido para redes aleatórias, com probabilidade uniforme de arestas $p$. No Apêndice 1, discutimos os pormenores da questão de ensemble de grafos aleatórios, ou melhor, redes aleatórias. Para fins práticos, substituímos o elemento de matriz $A_{i j}$ pelo seu valor esperado $\left\langle A_{i j}\right\rangle=p\left(1-\delta_{i j}\right)$. Ora, mas esse valor de elemento de matriz é proporcional aquele do grafo completo. Portanto, a equação correspondente requer apenas a substituição $\beta \rightarrow \beta p$ :

$$
\frac{d \rho}{d t}=(p \beta) \rho(1-\rho)-\gamma \rho
$$

A multiplicação do lado direito da Eq. (3.46) por $N / N$ recupera a formulação mais tradicional

$$
\frac{d \rho}{d t}=\beta\left(\frac{\langle k\rangle}{N}\right) \rho(1-\rho)-\gamma \rho,
$$

onde $\langle k\rangle=p N$ é o grau médio da rede. Esse resultado é intuitivo pois, grosso modo, produz o número de reprodução básico $R_{0}=\langle k\rangle(\beta / \gamma)$ por um fator que contempla o número de conexões disponíveis. A inclusão de redes ainda mais complexas já foi extensivamente analisada por Pastor-Satorras, Vespignani e Boguná $[1,21,58$, 89], e envolve a distribuição de probabilidade de graus. A fim de retornar aos nossos modelos baseados em agentes, encerramos a discussão acerca dos modelos compartimentais.

Voltamos à Eq. (3.42a) para discutir uma abordagem introduzida e desenvolvida majoritariamente por van Mieghem e colaboradores [112-117]. Em sua forma despida e para grafos arbitrários, a Eq. (3.44a) vale

$$
\begin{aligned}
n_{\mu k}= & \left\langle C_{\mu}\left|\hat{n}_{k}\right| C_{\mu}\right\rangle \\
-\frac{d}{d t}\langle n\rangle= & \frac{\beta}{N} \sum_{\mu \nu} \sum_{i j k} A_{i j} P_{\nu}\left[\delta_{\mu \nu} n_{\nu k}\left(1-n_{\nu i}\right) n_{\nu j}-n_{\mu k}\left\langle C_{\mu}\left|\hat{\sigma}_{i}^{+}\right| C_{\nu}\right\rangle n_{\nu j}\right]+ \\
& \gamma \sum_{\mu \nu} \sum_{i k} P_{\nu}\left[\delta_{\mu \nu} n_{\nu k} n_{\nu i}-n_{\mu k}\left\langle C_{\mu}\left|\hat{\sigma}_{i}^{-}\right| C_{\nu}\right\rangle\right] .
\end{aligned}
$$

Novamente, temos as seguintes valores esperados para operadores não-diagonais:

$$
\begin{aligned}
& \left\langle C_{\mu}\left|\hat{\sigma}_{i}^{+}\right| C_{\nu}\right\rangle=\delta_{n_{\mu}, n_{\nu}+1}\left(1-n_{\nu i}\right), \\
& \left\langle C_{\mu}\left|\hat{\sigma}_{i}^{-}\right| C_{\nu}\right\rangle=\delta_{n_{\mu}, n_{\nu}-1} n_{\nu i} .
\end{aligned}
$$


Substituindo esses resultados na Eq. (3.48b), obtemos

$$
\frac{d}{d t}\langle n\rangle=\frac{\beta}{N}\left[\sum_{\mu} \sum_{i, j} P_{\mu} A_{i j}\left(1-n_{\mu i}\right) n_{\mu j}\right]-\gamma\langle n\rangle .
$$

A aproximação de campo médio para $N$ equações entrelaçadas, assim denominada por van Mieghem em 2011 [113], é obtida a partir da Eq. (3.50) assumindo-se

$$
\begin{aligned}
\bar{n}_{i} & \equiv \sum_{\mu} P_{\mu}(t) n_{\mu i}, \\
\bar{n}_{i} \bar{n}_{j} & \approx \sum_{\mu} P_{\mu}(t) n_{\mu i} n_{\mu j},
\end{aligned}
$$

isto é, $n_{\mu j} \approx \bar{n}_{j}$ é muito bem representado pelo seu valor médio, quando contidas em contribuições quadráticas. Essa aproximação, é muito parecida com a aproximação de Bethe-Peierls $[102,118]$ (para a estatística $\left\langle\bar{n}_{i}\right\rangle$ ) da Mecânica Estatística, mais conhecida como aproximação de campo médio. Não confundir com as aproximações de campo médio relativas aos grafos completos e tampouco àquela da variância nula. A confusão, embora não proposital, tem lá seu lado lúdico. De acordo com a aproximação acima, a Eq. (3.50) torna-se

$$
\frac{d\langle n\rangle}{d t}=\frac{\beta}{N}\left[\sum_{i j} A_{i j}\left(1-\bar{n}_{i}\right) \bar{n}_{j}\right]-\gamma \sum_{i} \bar{n}_{i} .
$$

A outra parte da aproximação sobre a assunção que a Eq. (3.52) pode ser reescrita a partir da soma de $N$ equações diferenciais acopladas,

$$
\frac{d \bar{n}_{i}}{d t}=-\frac{\beta}{N}\left[\sum_{j} A_{i j} \bar{n}_{i} \bar{n}_{j}-\left(\kappa_{i}-\Gamma\right) \bar{n}_{i}\right],
$$

onde $\kappa_{i}=\sum_{j} A_{i j}$ é o grau (número de coordenação) do $i$-ésimo vértice.

Para aqueles já versados nas técnicas da Mecânica Estatística, especialmente no contexto de fenômenos cooperativos e transições críticas de fase [32, 33, 54], ao ver uma aproximação do tipo Bethe-Peierls também se espera equações de autoconsistência. Até o momento, elas tem sido negligenciadas e não foram documentadas em publicações relevantes. A razão disto se dá devido à simplicidade do sistema de equações acopladas, que permite a inclusão de um número elevado de agentes, e concorda com resultados numéricos para alguns problemas [117]. Por outro lado, a hipótese de independência das variáveis $\bar{n}_{k}$ não é algo trivial, muito menos intuitivo: essa hipótese não foi feita a partir da decomposição em termos de autofunções 
ortogonais, circunstância sobre a qual a separação em $N$ equações seria imediata. Outro ponto que merece destaque é a questão da equação análoga para a variância: neste caso, há certa ambiguidade quanto a aplicação da aproximação já que a termos cúbicos também contribuem.

Podemos aprender um pouco mais sobre a Eq. (3.53) por meio de sua análise operatorial. Considere o vetor $|\bar{n}\rangle=\sum_{i} \bar{n}_{i}|i\rangle$ tal que

$$
\begin{gathered}
\hat{\kappa}=\sum_{i} \kappa_{i}|i\rangle\langle i|, \\
\hat{A}=\sum_{i j} A_{i j}|i\rangle\langle j|, \\
\hat{\rho}(\bar{n})=\sum_{i} \bar{n}_{i}|i\rangle\langle i|, \\
\hat{B}(\bar{n})=\frac{\beta}{N}[\hat{\kappa}-\hat{\rho}(\bar{n}) \hat{A}] .
\end{gathered}
$$

O operador $\hat{\rho}$ tem papel análogo ao da matriz densidade [41] da Mecânica Quântica para estados puro. A ação do operador $\hat{B}(\bar{n})-\gamma \mathbb{1}$ sobre o vetor $|\bar{n}\rangle$ reproduz o lado direito da Eq.(3.53), que resulta na expressão

$$
\frac{d}{d t}|\bar{n}\rangle=[\hat{B}(\bar{n})-\gamma]|\bar{n}\rangle
$$

que porventura também é uma equação de auto-consistência. Em particular, no equilíbrio, temos uma equação de autovalor e autovetor:

$$
\hat{B}\left(\bar{n}_{e q}\right)\left|\bar{n}_{e q}\right\rangle=\gamma\left|\bar{n}_{e q}\right\rangle
$$

Podemos extrair mais informações da Eq. (3.55) se considerarmos a matriz Laplaciana $\hat{L}$ de grafos, cujos elementos de matriz são $L_{i j} \equiv\left(\delta_{i j} \kappa_{i}-A_{i j}\right)$. Suas propriedades espectrais são frequentemente utilizadas para caracterizar grafos, mais precisamente, suas componentes topológicas, partições, caminhos fechados, etc. Uma propriedade espectral muito útil de $\hat{L}$ é que todos os seus autovalores satisfazem $\lambda \geqslant 0$, isto é, trata-se de uma matriz semi-postitiva definida. Em termos de $\hat{L}$, o operador $\hat{B}$ vale

$$
\hat{B}(\bar{n})=\frac{\beta}{N}[(\hat{\mathbb{1}}-\hat{\rho}) \hat{\kappa}+\hat{\rho} \hat{L}] .
$$

Note que o produto de operadores $(\hat{\mathbb{1}}-\hat{\rho}) \hat{\kappa}$ é diagonal na base dos vértices, $|i\rangle$. Substituindo esse resultado na Eq. (3.55), temos

$$
\frac{d}{d t}|\bar{n}\rangle=\frac{\beta}{N}\{\hat{\rho} \hat{L}+[(1-\hat{\rho}) \hat{\kappa}-\Gamma]\}|\bar{n}\rangle .
$$


Existem alguns casos de interesse que valem a pena comentar. No primeiro caso, examina-se a possibilidade de que todos os agentes do sistema se tornem infectados. Nesse caso, $\hat{\rho} \approx \mathbb{1} \mathrm{e}$, portanto, o vetor $|\bar{n}\rangle$ pode ser expresso como combinação linear dos autovetores $\left|l_{k}\right\rangle$ de $\hat{L}$, com autovalores $\ell_{k}$. Para facilitar a discussão, façamos $\hat{L}|\bar{n}\rangle=\ell|\bar{n}\rangle$. Tal vetor é um estado de equilíbrio se e somente se $\ell=\Gamma$. De fato, essa predição é observada no modelo SI $(\Gamma=0)$, incluindo os casos onde o grafo contém diversos subgrafos disjuntos. Cada subgrafo disjunto corresponde a uma comunidade isolada, e os autovalores $\ell=0$ correspondem a vetores com coeficientes $n_{k}=1$ se $k$ pertence ao subgrafo, nulos caso contrário.

O caso oposto, quando a densidade de infectados $n_{i} \ll 1$, o operador $\hat{\rho} \approx \hat{0}$. Logo, cada vértice satisfaz $n_{i}(t)=n_{i}(0) \exp \left[(\beta / N)\left(\kappa_{i}-\Gamma\right) t\right]$. Portanto, o número de infectados $n_{i}$ do vértice $i$ decai exponencialmente se $\kappa_{i}<\Gamma$ ou se mantém constante se $\kappa_{i}=\Gamma$. Vale a pena lembrar que essa equação leva em conta a heterogeneidade das redes de contanto.

Por fim, mencionamos que as Eqs. (3.52) e (3.53) partilham muito em comum o formalismo da teoria do funcional densidade, do inglês Density Functional Theory (DFT) [119]. Aqui, o objetivo é resolver a Eq. (3.52). Primeiro, determinam-se os valores de $\bar{n}_{i}(t)$, para $t$ fixo, por meio da Eq. (3.53). Tais valores são substituídos nas $N$ equações $\bar{n}_{i}=\sum_{\mu} P_{\mu} n_{\mu i}$, a partir das quais determina-se uma possível solução para as probabilidades $P_{\mu}(t)$. Estas então são substituídas na Eq. (3.52). Claro, essa tarefa se restringe à tarefas computacionais.

Contudo, ao contrário da DFT que conta com os teoremas Hohenberg-Kohn [120], essa proposta carece um mecanismos de controle, que descreva a convergência das soluções propostas para a solução correta. Baseados nos resultados obtidos por van Mieghem e pela interpretação operatorial apresentada nesta seção, acreditamos que seja possível deduzir um teorema equivalente.

\subsection{Norma do vetor probabilidade}

A distribuição de probabilidade de configurações $P_{\mu}(t)$ desempenha papel fundamental para a caracterizar do sistema, bem como suas propriedades. Por outro lado, cada $P_{\mu}(t)$ diz respeito somente à sua configuração correspondente. Falta, portanto, alguma medida para avaliar o sistema como um todo, que por falta de 
melhor opção denominamos global. Já vimos que a conservação da probabilidade total, $\sum_{\mu} P_{\mu}(t)=1$, impede seu uso como uma propriedade global. Como próxima alternativa, escolhemos a norma do vetor probabilidade,

$$
|P(t)|^{2}=\langle P(t) \mid P(t)\rangle=\sum_{\mu} P_{\mu}^{2}(t)
$$

como medida global do sistema. Ao contrário da soma total das probabilidades, $|P(t)|^{2}$ não é conservada ao longo do tempo, exceto no regime de equilíbrio. Note que estabelecemos um critério para definir equilíbrio de um processo estocástico. A Figura (3.13) mostra o comportamento de $|P(t)|^{2}$ para o modelo SIS.

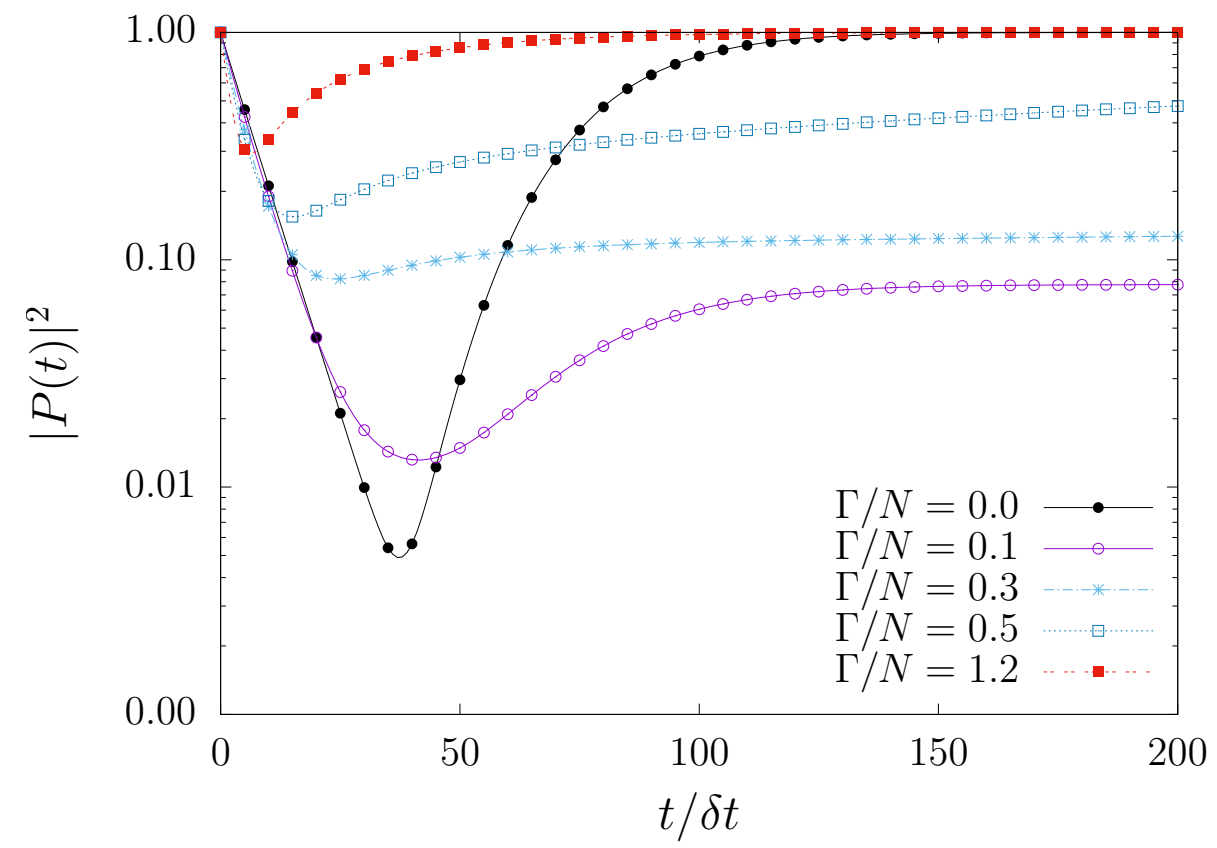

Figura 3.13: $|P(t)|^{2}$ do modelo SIS com $N=12$ para vários valores de acoplamento $\Gamma$. A condição inicial é $P_{1}(0)=1$. Para todos os acoplamentos $\Gamma$, o sistema parte de um cenário com norma unitária. Com a evolução do sistema, outras configurações tornam-se prováveis, diminuindo o valor de $|P(t)|^{2}$ até atingir seu valor mínimo. Neste ponto, a incerteza quanto à configuração do sistema atinge seu valor máximo, no tempo $t=t_{c}$. A evolução do sistema prossegue até que o equilíbrio do sistema é atingido, onde $(d / d t)|P(t)|^{2}=0$. No equilíbrio, o valor de $|P(t)|^{2}$ depende da particular composição de estados estacionários. Reprodução da Ref. [86] sob permissão acadêmica.

Mas quais os motivos que sugerem que $|P(t)|^{2}$ de fato execute sua finalidade como propriedade descritiva do sistema? Como visto anteriormente, o número de infectados no modelo SIS corresponde a $\langle n\rangle=\sum_{\mu} n_{\mu} P_{\mu}(t)$. Vimos também que essa média pode ser calculada a partir de $\langle n\rangle=\langle\Phi|\hat{n}| P(t)\rangle=\langle P(t)|(\hat{\Phi} \hat{n})| P(t)\rangle$, onde $\hat{\Phi}=|\Phi\rangle\langle\Phi|$. À primeira vista, essas manipulações matemáticas parecem redundan- 
tes posto que o valor numérico nunca muda. Contudo, com o auxílio do operador $\hat{\Phi}$, a estimação de estatísticas ganha uma nova interpretação para processos estocásticos: dado o operador $\hat{\mathcal{O}}$, sua média é o valor esperado do produto de operadores $\langle\mathcal{O}\rangle=\langle P(t)|\hat{\Phi} \hat{\mathcal{O}}| P(t)\rangle$. De acordo, o produto escalar $\langle P(t) \mid P(t)\rangle$ adquire inesperada relevância, já que todas as médias são calculadas a partir dele.

Naturalmente, a norma apresenta simetria por transformações unitárias $\hat{U}$ ou mudanças de base -, pois $\langle P(t) \mid P(t)\rangle=\left\langle P(t)\left|\hat{U}^{-1} \hat{U}\right| P(t)\right\rangle=\left\langle P^{\prime}(t) \mid P^{\prime}(t)\right\rangle$. Essa propriedade é excelente já que o sistema não deve depender da base sobre o qual é modelado. Com essa observação em mente, resta procurar a equação que descreve a evolução de $|P(t)|^{2}$. Dado o conhecimento prévio da Eq. (3.34), basta calcular a derivada de $|P(t)|^{2}$ :

$$
\frac{1}{2} \frac{d}{d t}|P(t)|^{2}=-\langle P(t)|\hat{\mathcal{H}}| P(t)\rangle,
$$

onde o operador $\hat{\mathcal{H}}=(1 / 2)\left(\hat{H}+\hat{H}^{\dagger}\right)$ é Hermitiano por construção.

Como todo operador Hermitiano, os autovalores $\left\{\Lambda_{\mu}\right\}$ são reais, embora não necessariamente semi-positivos como acontece com os autovalores de $\hat{H}$, como mostra a Figura 3.14. Porém, existe uma grande vantagem: os autovetores $\left\{\left|\psi_{\mu}\right\rangle\right\}$ formam uma base ortonormal, com vetores à direita e à esquerda relacionados pela simples conjugação Hermitiana. Esse conjunto de $2^{N}$ vetores gera o espaço de Hilbert. Do ponto de vista numérico, também há vantagens, uma vez que bibliotecas de diagonalização de matrizes exploram a hermiticidade e para o armazenamento eficiente de elementos de matriz.

Seguindo a praxe, a combinação linear do vetor probabilidade na base dos dos autovetores de $\hat{\mathcal{H}}$ é

$$
|P(t)\rangle=\sum_{\mu} g_{\mu}(t)\left|\psi_{\mu}\right\rangle
$$

Ao contrário da distribuição de probabilidade $P_{\mu}(t)$, que é sempre positiva ou nula para qualquer $\mu=0,1, \ldots, 2^{N}-1$, os coeficientes $g_{\mu}(t)$ são números complexos e, portanto, não podem ser interpretados como probabilidades. Apesar disso, os coeficientes $g_{\mu}(t)$ se relacionam com as probabilidades $P_{\mu}(t)$ por meio da expressão

$$
P_{\mu}(t)=\sum_{\nu} g_{\nu}(t)\left\langle C_{\mu} \mid \psi_{\nu}\right\rangle
$$

Note que a conservação da probabilidade total também introduz um vínculo para 


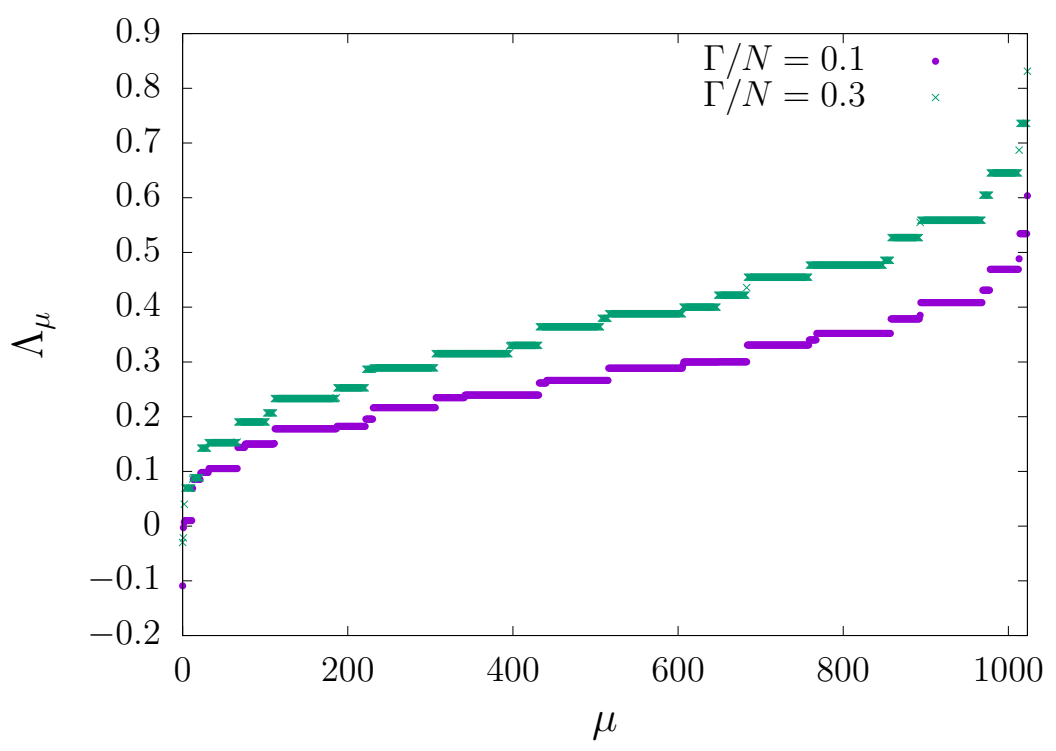

Figura 3.14: Autovalores ordenados de $\mathcal{H}$ com $N=10$. Os círculos (cruzes) representam os $\Lambda_{\mu}$ para o acoplamento $\Gamma / N=0.1(0.3)$. Figura reproduzida da Ref. [86] sob permissão acadêmica.

os coeficientes $g_{\mu}(t)$, pois

$$
\sum_{\mu} P_{\mu}(t)=\sum_{\mu \nu} g_{\nu}\left\langle C_{\mu} \mid \psi_{\nu}\right\rangle=\sum_{\nu} g_{\nu}(t)\left\langle\Phi \mid \psi_{\nu}\right\rangle=1
$$

Por meio dos autovetores $\left|\psi_{\mu}\right\rangle$ e autovalores $\Lambda_{\mu}$, deriva-se uma equação para o módulo quadrado dos coeficientes $g_{\mu}(t)$, a partir da Eq. (3.60):

$$
\sum_{\mu}\left(\frac{1}{2} \frac{d}{d t}+\Lambda_{\mu}\right)\left|g_{\mu}(t)\right|^{2}=0
$$

Vale a pena enfatizar que muito embora essa equação tenha sido deduzida num contexto de modelos epidêmicos, sua validade se estende para qualquer processo Markoviano. Essa equação assim como seus resultados, metodologias relacionadas e conclusões foram recentemente publicadas na Ref. [86]. No que segue, exploramos alguns de seus resultados mais importantes.

O primeiro resultado, e talvez o mais impactante apesar de sua simplicidade, consiste na análise da Eq. (3.64) quando $(d / d t)|P(t)|^{2}=0$. Existem duas situações onde isso acontece, a saber, quando o sistema atinge o regime de equilíbrio; ou quando o sistema atinge a máxima incerteza ao longo de sua evolução temporal (Figura 3.15). A fim de especificar um exemplo concreto, considere a curva correspondente ao modelo SIS com $\Gamma=0$ na Figura 3.13. O equilíbrio ocorre para $t>150 \delta t$, donde se observa a conservação de $|P(t)|^{2}$. Pouco antes de $t=50 \delta t$, 


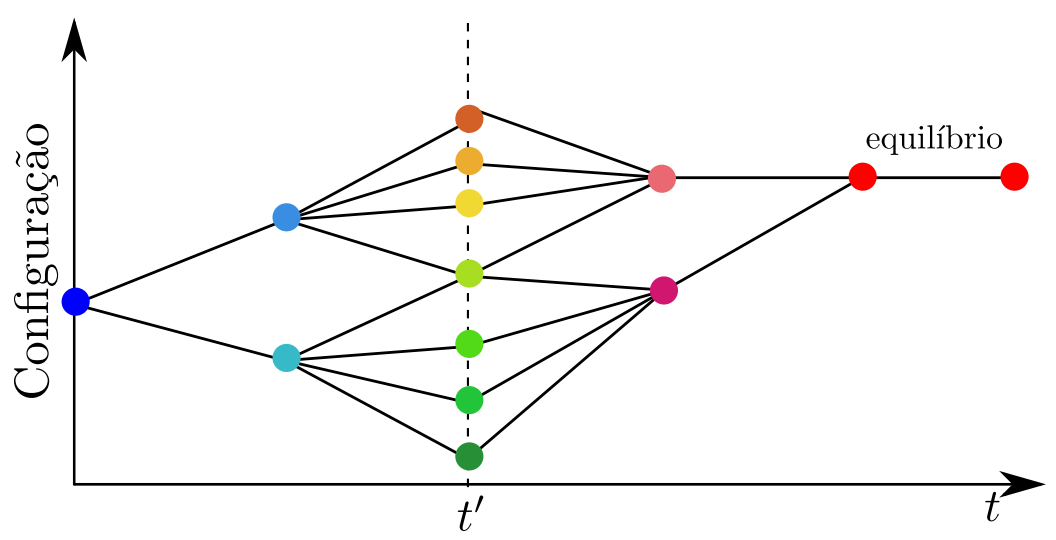

Figura 3.15: Processo estocástico discreto arbitrário. A probabilidade da configuração inicial em $t=0$ é unitária. Nos instantes de tempos subsequentes, a probabilidade de se encontrar o sistema em outras configurações aumenta. Em determinado tempo $t^{\prime}$, o número de configurações disponíveis torna-se máximo. As probabilidades associadas a estas configurações são distribuídas de tal maneira que $\sum_{\mu} P_{\mu}^{2}\left(t^{\prime}\right)$ atinge seu menor valor. Para $t>t^{\prime}$, o sistema eventualmente converge para o equilíbrio, aqui descrito por uma única configuração estacionária.

durante o regime transiente, $|P(t)|^{2}$ atinge seu valor mínimo devido a máxima disponibilidade de configurações de $N$ agentes. ${ }^{21}$

Em ambos os casos, a Eq. (3.64) assume uma forma que depende tão somente de elementos espectrais de $\hat{\mathcal{H}}$ :

$$
\sum_{\mu}\left|\tilde{g}_{\mu}\left(t^{\prime}\right)\right|^{2} \Lambda_{\mu}=0
$$

onde $t^{\prime}$ indica que ou o sistema se encontra no equilíbrio ou no ponto de máxima incerteza. Nesta formulação, a Eq. (3.65) é uma função quadrática multivariada, devido aos diversos coeficientes $\left|g_{\mu}\right|^{2}$. Se por ventura os autovalores $\Lambda_{\mu}$ são conhecidos, então a Eq. (3.65) pode ser resolvida e a solução representa o sistema em $t^{\prime}$. Em particular, se $\hat{H}$ for semi-positiva definida, então o equilíbrio é também o estado de máxima incerteza. Isto decorre da propriedade $\Lambda_{\mu} \geqslant 0$ : apenas os coeficientes correspondentes aos autovalores nulos podem adquirir valores não-nulos. ${ }^{22}$ Em geral, para fenômenos fora do equilíbrio, incluindo aí os modelos epidêmicos, os operadores $\hat{\mathcal{H}}$ não são semi-positivos definidos. Portanto, seus autovalores podem ser negativos, como é o caso do modelo SIS (Figura 3.14).

\footnotetext{
${ }^{21}$ Para complementar com um exemplo mais tradicional, considere novamente o movimento browniano unidimensional que se desloca ao longo de uma reta $(x \in[-\infty, \infty])$. A norma descreve a seguinte curva $|P(t)|^{2}=(8 \pi D t)^{-1 / 2}$. Por ser uma lei de potência, a norma decai monotonicamente. Se o intervalo de integração for finito mas muito maior que o passo da partícula, então a posição da partícula é uniformemente distribuída ao longo do segmento de reta.

${ }^{22}$ Recorrendo ao modelo browniano, seus autovalores são $\Lambda_{k}=D k^{2}$, onde $k$ é o número de onda. A única solução possível para a Eq. (3.65) é o estado de momento nulo, que por sua vez corresponde à distribuição uniforme $P(x, t)=L^{-1}$. Aqui, $L \delta x$ representa o comprimento físico do segmento de reta.
} 


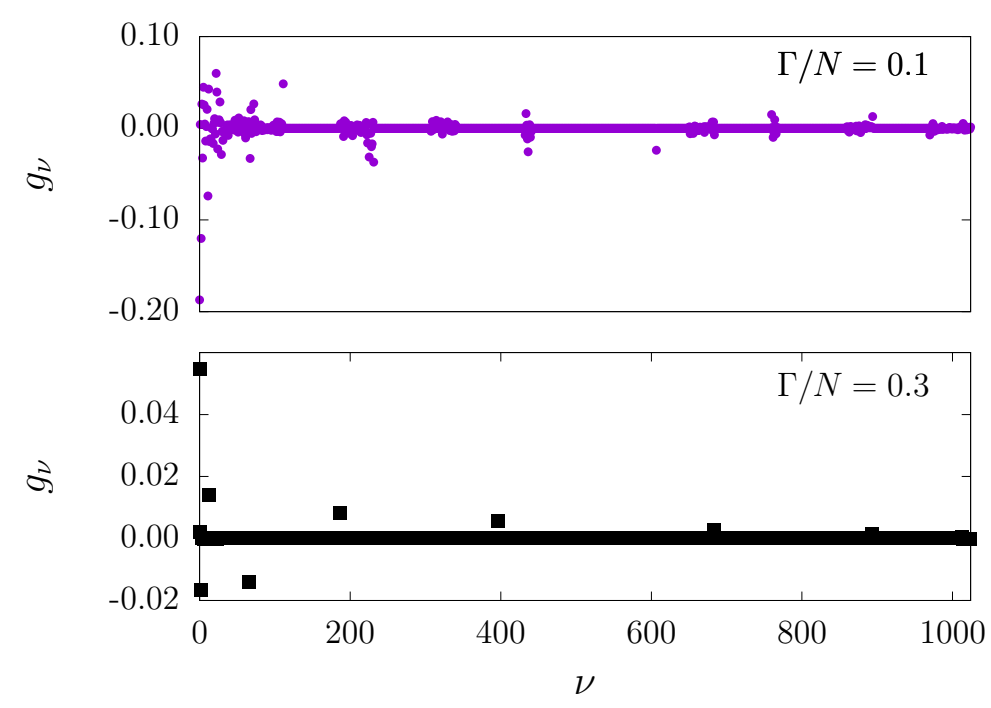

Figura 3.16: Solução $g_{\mu}\left(t^{\prime}\right)$ do modelo SIS com $N=10$ agentes. O instante de tempo $t=t^{\prime}$ caracteriza-se pela ocorrência do maior número de configurações disponíveis durante o transiente. Após $t^{\prime}$, o sistema converge para o equilíbrio. Os valores de $\Gamma / N=0.1$ e 0.3 explicitam a mudança significativa para a distribuição dos coeficientes $g_{\mu}\left(t^{\prime}\right)$ conforme o valor de $\Gamma$. Figura reproduzida da Ref. [86] sob permissão acadêmica.

Se os autovalores $\Lambda_{\mu}$ são conhecidos - ou estimados segundo alguma metodologia adequada - então a solução da Eq. (3.65), com o vínculo Eq. (3.63), se reduz a um problema numérico. A Figura 3.16 mostra a solução da Eq. (3.65) para dois valores distintos de acoplamento $\Gamma$. Esses resultados foram obtidos utilizando-se a subrotina f solve do software Octave [121], vulgarmente conhecido como força bruta, uma vez que a convergência para solução correta depende da escolha adequada da solução inicial. Para nossa finalidade, que é demonstrar que existem soluções para a Eq. (3.65) e estas são corretas, esse método é suficiente. Uma técnica mais eficaz para se determinar as soluções é por meio de métodos de otimização. Para isso, define-se a função objetivo

$$
F_{r}(\{g\})=\exp \left[-\left(\sum_{\mu} \Lambda_{\mu}|g|_{\mu}^{2}\right)^{r}-\eta\left|\sum_{\nu} g_{\nu} \Phi_{\nu}-1\right|^{2}\right],
$$

com $r$ inteiro positivo e $\Phi_{\nu}=\left\langle\Phi \mid \psi_{\nu}\right\rangle$. O acoplamento $|\eta| \gg 1$ enfatiza o vínculo da probabilidade total. Em particular, para $r=1$ e $g_{\mu}$ reais, o problema se resume à maximização de gaussianas. Para $r=2$, a função objetivo fica limitada $0 \leqslant F_{2} \leqslant 1$. Outro caso muito conveniente é $\eta=\imath|\eta|$, pois minimiza-se o funcional no plano real e no plano complexo, evitando-se a mistura patológica entre o termo que corresponde à Eq. (3.65) com o termo que expressa o vínculo. 
Para $N \gg 1$, o espectro de autovalores torna-se denso e pode ser representado pela densidade espectral $\rho(\Lambda)$, enquanto a variável $z(\Lambda)$ corresponde ao limite do contínuo para $\left|g_{\mu}\right|^{2}$. Sob tais circunstâncias, a Eq. (3.65) descreve uma equação integral para $z(\Lambda)$, a saber,

$$
\int_{-\infty}^{\infty} d \Lambda \Lambda \rho(\Lambda) z(\Lambda)=0
$$

Apesar de nossos esforços para apresentar $|P(t)|^{2}$ como uma variável dinâmica global, ainda resta a questão a respeito de sua interpretação física. Questões como "Qual seu significado? É possível medir $|P(t)|^{2}$ diretamente? Como seus valores afetam as outras propriedades globais?" são naturais e merecem respostas adequadas. De fato, logo após a dedução das Eqs. (3.64) e (3.65), surgiram duas interpretações distintas e conflitantes para $|P(t)|^{2}$, conforme relatamos a seguir. Uma interpretação baseou-se nos conceitos de Ação e Lagrangeana da Mecânica Clássica $[38,39]$, para as variáveis $z_{\mu}(t) \equiv\left|g_{\mu}(t)\right|^{2}, \dot{z}_{\mu}(t) \equiv(d / d t)\left|g_{\mu}(t)\right|^{2}$. Separando a Eq. (3.64) em duas somatórias e quadrando ambos os lados da igualdade, temos

$$
\frac{1}{4} \sum_{\mu \nu} \dot{z}_{\mu} \dot{z}_{\nu}=\sum_{\mu \nu} \Lambda_{\mu} \Lambda_{\nu} z_{\mu} z_{\nu}
$$

A integração desta expressão resulta numa constante que, por conveniência chamamos de ação e representamos por $\mathcal{S}$,

$$
\mathcal{S} \equiv \sum_{\mu} \int_{t_{0}}^{t_{f}} d t\left[\frac{1}{4} \sum_{\mu \nu} \dot{z}_{\mu} \dot{z}_{\nu}-\sum_{\mu \nu} \Lambda_{\mu} \Lambda_{\nu} z_{\mu} z_{\nu}\right] .
$$

A partir da definição formal de ação, a comparação com $\mathcal{S}$ produz a Lagrangeana

$$
\mathcal{L} \equiv \frac{1}{4} \sum_{\mu \nu}\left(\dot{z}_{\mu} \dot{z}_{\nu}-4 \Lambda_{\mu} \Lambda_{\nu} z_{\mu} z_{\nu}\right)+\zeta(t) \sum_{\mu}\left(z_{\mu}^{1 / 2} \mathrm{e}^{\imath \varphi_{\mu}} \Phi_{\mu}-1\right)
$$

onde $\zeta(t)$ é o multiplicador de Lagrange correspondente ao vínculo da conservação de probabilidade total, $\Phi_{\mu}=\left\langle\Phi \mid \psi_{\mu}\right\rangle$ e $g_{\mu}(t)=z_{\mu}^{1 / 2} \mathrm{e}^{\imath \varphi_{\mu}}$. Neste ponto, convém utilizar o princípio variacional e obter as equações de movimento [38]. A forma bilinear de $\mathcal{L}$ sugere que existem transformações canônicas convenientes, que facilitem a resolução das equações de movimento com vínculo.

Uma descrição alternativa emprega o conceito de integrais de trajetórias de Feynman [122], que decorre naturalmente da definição da ação $\mathcal{S}$ :

$$
\mathcal{Z}\left(\beta^{\prime}\right)=\int \prod_{\mu} \mathcal{D} z_{\mu} \mathcal{D} \varphi_{\mu} \mathrm{e}^{-\beta^{\prime} \mathcal{S}} \mathrm{e}^{-\beta^{\prime} \zeta^{\prime}\left(\sum_{\mu} \sqrt{z_{\mu}} \mathrm{e}^{2 \varphi} \mu \Phi_{\mu}-1\right)^{2}}
$$


onde $\beta^{\prime}$ tem dimensão inversa à da ação e $\zeta^{\prime}$ tem dimensão de ação. Aqui, relaxamos o vínculo de conservação de probabilidade mas, simultaneamente, garantimos que é satisfeito se $\beta^{\prime} \zeta^{\prime} \gg 1$. A ideia, portanto, consiste em explorar $\mathcal{Z}$ como função de partição do problema, como habitual em Teorias de Campos e Mecânica Estatística no equilíbrio $[17,32,79]$. Destacamos que o cálculo de $\mathcal{Z}$ para $\zeta^{\prime}=0$ é bem documentado em referencias relevantes $[54,79,123]$. Do ponto de vista teórico, essa interpretação é fascinante pois descreve um fenômeno fora do equilíbrio por meio de ferramentas do equilíbrio $(\mathcal{Z})$ com uma temperatura efetiva $\beta^{\prime}$.

Apesar do forte apelo teórico, a interpretação acima não produz uma conexão imediata com os resultados que temos em mãos. Especialmente com o regime de equilíbrio real ou com aquele no instante de tempo $t=t^{\prime}$, durante o transiente, no qual $|P(t)|^{2}$ assume valor mínimo. Por esse motivo, adotamos formalmente a interpretação abaixo, fortemente baseada no conceito de entropia. ${ }^{23}$ Seja $\langle P\rangle_{R}=$ $|P(t)|^{2}=\sum_{\mu} P_{\mu} P_{\mu}$ o estimador para a probabilidade média de configurações. Na Física Estatística, o exemplo mais simples de $\langle P\rangle_{R}$ fundamenta-se sobre a Hipótese de Boltzmann [53], isto é, todos os microestados possuem mesma probabilidade $\langle P\rangle_{R}=2^{-N}$. Decorre também a definição de entropia de Boltzmann $S_{B}=-N \ln 2$, onde utilizamos uma escala normalizada tal que a constante de Boltzmann vale $k_{b}=$ 1. No nosso caso, entretanto, as diversas configurações $\left|C_{\mu}\right\rangle$ não são equiprováveis para $t$ arbitrário.

Como nossa escolha para entropia deve valer para qualquer processo Markoviano, incluindo os casos cobertos pela Hipótese de Boltzmann, utilizamos a entropia quadrática de Rényi

$$
S_{R} \equiv-\ln \sum_{\mu} P_{\mu}^{2}=-\ln \langle P\rangle_{R}
$$

que faz parte de uma família de entropias generalizadas [124]. Um das propriedades satisfeitas pela entropia quadrática de Rényi é a aditividade. Por exemplo, considere o caminhante aleatório, após atingir o equilíbrio, numa região espacial delimitada e com volume $a^{3} V$, com $a^{3}$ sendo o volume físico da célula unitária. Por ser um único caminhante, $P(\vec{x}, t)=V^{-1}$ com $P_{1}^{2}=V\left(1 / V^{2}\right)=V^{-1}$, que resulta na entropia

\footnotetext{
${ }^{23}$ Cabe aqui comentar que essa decisão foi excruciante. De um lado, todo o formalismo Lagrangeano para descrever as equações de movimento da norma quadrada, o formalismo de Feynman e sua conexão com Teorias de Campos. Do outro lado, o conceito intuitivo de entropia. De fato, em conversa privada, Prof. Dr. George Cardoso, um dos colaboradores desse assunto, apresentou nosso trabalho ao Prof. Eugene Stanley - mais conhecido na área de Física Estatística como "o Stanley" - que prontamente também interpretou o ponto de mínimo da norma quadrada como o ponto de máxima entropia.
} 
$S_{1}=\ln V$. Se considerarmos $N$ caminhantes independentes, $P_{N}^{2}=V^{-N}$ tal que $S_{N}=N S_{1}$, ou seja, a entropia total é a soma das entropias individuais.

Em nossa argumentação, a seleção em favor da entropia de Rényi em detrimento de outras entropias generalizadas - como a de Tsallis [125], $S_{T}=1-|P|^{2}$, com parâmetro não-extensivo $q=2$ - se fundamentou tão somente na aditividade da entropia para processos Markovianos e independentes. Essa classe de sistemas são mais familiares na Termodinâmica. Contudo, um argumento semelhante também poderia ser feito, digamos, em favor de sistemas não-extensivos, onde a entropia de Tsallis seria melhor candidata. De fato, a escolha da entropia não é questão fechada. Uma alternativa para solucionar esse dilema, seria a entropia efetiva

$$
\left|S_{E}\right|=\sqrt{\cos ^{2}(\theta) S_{R}^{2}+\sin ^{2}(\theta) S_{T}^{4}}
$$

onde o ângulo $\theta$ atua como uma medida sobre o caráter não-extensivo do sistema. Assim, a entropia efetiva é equivalente à formulação entrópica no plano complexo, $S_{E}=\left|S_{E}\right| \mathrm{e}^{\imath \theta}$, onde o eixo real (imaginário) atribui o caráter aditivo (não-aditivo) à entropia. Note que para $\theta=0$ e dois sistemas $A$ e $B$, respectivamente, temos $S_{E}(A+$ $B)=S_{R}(A)+S_{R}(B)$. Ao contrário, para $\theta=\pi / 2$, temos $S_{E}(A+B)=S_{T}^{2}(A)+$ $S_{T}^{2}(B)=S_{T}^{2}(A+B)-2 S_{T}(A) S_{T}(B)$. Contudo, a viabilidade do parâmetro $\theta$, bem como os efeitos da adoção da entropia efetiva, encontram-se numa fase hipotética, devido à ausência de evidências concretas. Por esse motivo, prosseguimos com a entropia de Rényi da Eq. (3.72).

Como fechamento dessa seção, enfatizamos que as Eqs. (3.64) e (3.65) descrevem um novo método para se resolver processos estocásticos. Em parte porque o papel dos geradores temporais $H$, que podem ser não-simétricos, é substituído pelo operador simétrico $\mathcal{H}$. Os autovalores de $\mathcal{H}$ são então utilizados para determinar os regimes de equilíbrio e o ponto de máxima entropia, durante o regime transiente, por meio da equação algébrica Eq. (3.65). O grande "porém" é a determinação dos autovalores $\Lambda_{\mu}$ : trata-se de um problema de muitos-corpos. Por sorte, estes mesmo problemas também são recorrentes em outras áreas da Física e podemos emprestálas sem hesitação. No que segue, discutimos alguns métodos e técnicas auxiliares oriundas da teoria de muitos-corpos, sistemas fortemente correlacionados e de ótica quântica, e suas aplicações na propagação de doenças infecciosas. 


\subsection{Teoria de Perturbação}

Muitas vezes sabemos diagonalizar um dado operador $\hat{\mathcal{H}}$ para um conjunto particular de acoplamentos, incluindo ai a distribuição de arestas. Se um dos acoplamentos, digamos o acoplamento $x$, sofrer um alteração $x+\delta x$, nada mais natural que expressar as novas soluções em termos daquelas já conhecidas. Diz-se que o novo autovalor (autovetor) é igual ao autovalor (autovetor) antigo mais correções. O que torna isso interessante é habilidade de organizar as correções como uma série de potência em $\delta x$. A teoria de perturbação mais simples é teoria de perturbação de Rayleigh-Schrödinger [40-42, 77].

Para o modelo SIS, o gerador simétrico é $\hat{\mathcal{H}}=\hat{\mathcal{H}}_{0}+\hat{\mathcal{H}}_{1}$, com

$$
\begin{aligned}
& \hat{\mathcal{H}}_{0}=\frac{\beta}{2 N} \sum_{k j} A_{j k}\left[2\left(1-\hat{n}_{j}\right) \hat{n}_{k}-\left(\hat{\sigma}_{j}^{+} \hat{n}_{k}+\hat{n}_{k} \hat{\sigma}_{j}^{-}\right)\right], \\
& \hat{\mathcal{H}}_{1}=\gamma \sum_{k j} \delta_{k j}\left(\hat{n}_{k}-\hat{\sigma}_{k}^{x}\right) .
\end{aligned}
$$

Aqui, $\hat{\sigma}_{k}^{x}=(1 / 2)\left(\hat{\sigma}^{+}+\hat{\sigma}^{-2}\right)$. Os elementos de matriz $A_{i j}$ compreendem tanto um único grafo como também os valores médios, estimados a partir do ensemble de grafos representantes da rede desejada (Apêndice 1). Uma breve inspeção do operador $\hat{\mathcal{H}}$ mostra que a simetria $U(1)$ - a conservação de partículas - é quebrada pela ação dos operadores localizados $\hat{\sigma}_{j}^{ \pm} \hat{n}_{k}$.

\subsubsection{Redes aleatórias}

Em geral, métodos perturbativos sempre se iniciam a partir de um caso onde a solução é conhecida. À primeira vista, $A_{i j}$ é o termo mais complicado da Eq. (3.74a), já que nada sobre o grafo ou rede fora especificado. Logo, utilizaremos $A_{i j}=1-\delta_{i j}$ para expressar o gerador simétrico $\hat{\mathcal{H}}_{\mathrm{MF}}$, que descreve o processo epidêmico no grafo completo. Apesar de sua simplicidade, frente à grafos mais complexos, o operador $\hat{\mathcal{H}}_{\mathrm{MF}}$ fornece pistas importantes, especialmente a respeito de simetrias globais. Para isso, define-se os operadores de momento angular de muitos corpos:

$$
\begin{aligned}
\hat{J}^{z} & =\sum_{k} \hat{n}_{k}-\frac{N}{2}, \\
\hat{J}^{ \pm} & =\sum_{k} \hat{\sigma}_{k}^{ \pm}, \\
\hat{J}^{x} & =\frac{1}{2} \sum_{k}\left(\hat{\sigma}_{k}^{+}+\hat{\sigma}_{k}^{-}\right),
\end{aligned}
$$


que são invariantes por permutações cíclicas dos vértices (ver Apêndice 2). A partir destes operadores, temos

$$
\frac{\hat{\mathcal{H}}_{\mathrm{MF}}}{\beta / N}=\frac{N}{2}\left(\frac{N}{2}+\Gamma\right)-\hat{J}^{z}\left(\hat{J}^{z}-\Gamma\right)-\frac{1}{2}\left(\hat{J}^{+} \hat{J}^{z}+\hat{J}^{z} \hat{J}^{-}\right)-\hat{J}^{x}\left(\frac{N}{2}+\Gamma\right) .
$$

Como se vê, o operador $\hat{\mathcal{H}}_{\text {MF }}$ potências tanto do operador $\hat{J}^{z}$ quanto do operador $\hat{J}^{x}$. Isso sugere o emprego da base de autovetores do operador de Casimir (quadrático) correspondente, $\hat{J}^{2}=\left(\hat{J}^{z}\right)^{2}+(1 / 2)\left\{\hat{J}^{+}, \hat{J}^{-}\right\}$, posto que satisfaz $\left[\hat{J}^{2}, \hat{J}^{x}\right]=\left[\hat{J}^{2}, \hat{J}^{z}\right]=$ 0. Consequentemente, $\left[\hat{\mathcal{H}}_{\mathrm{MF}}, \hat{J}^{2}\right]=0$.

Os autovetores $|j, m\rangle$ satisfazem $\hat{J}^{2}|j, m\rangle=j(j+1)|j, m\rangle$, com $j=$ $N / 2, N / 2-1, \ldots$ e $j \geqslant 0$, e $\hat{J}^{z}|j, m\rangle=m|j, m\rangle$, com $m=-j,-j+1, \ldots, j$. Portanto, existe uma combinação linear de $|j, m\rangle$ que diagonaliza $\hat{\mathcal{H}}_{\mathrm{MF}}$. A introdução destes autovetores também evidencia a conservação do número $j$, ou seja, a representação matricial de $\hat{\mathcal{H}}_{\mathrm{MF}}$ consiste em blocos disjuntos, cada um identificado por um valor de $j$, como ilustra a Figura (3.17). Como consequência, transições entre setores com diferentes valores de $j$ são proibidas. Cada bloco possui dimensão $d=2 j+1$

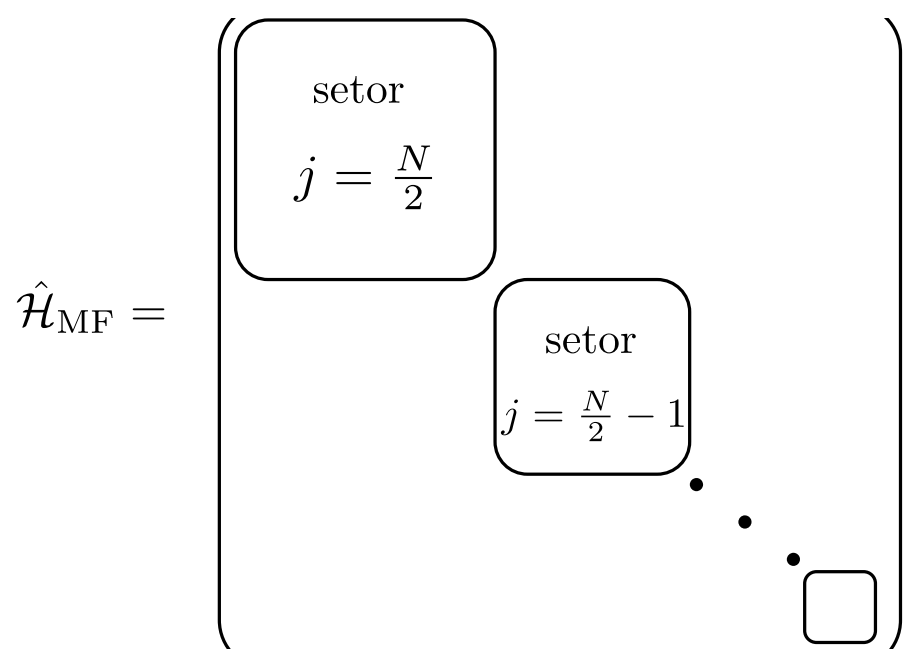

Figura 3.17: Conservação do momento angular total do operador $\hat{\mathcal{H}}_{\mathrm{MF}}$. A conservação do momento angular total implica na separação da representação matricial de $\hat{\mathcal{H}}_{\mathrm{MF}}$ em blocos disjuntos, na base $|j, m\rangle$. Cada bloco é univocamente identificado por um número $j$ correspondente, com dimensão $(2 j+1) \times(2 j+1)$.

A partir da Eq. (3.76), é fácil verificar que o operador $\hat{J}^{x}$ incrementa ou decrementa o valor de $m$ por uma unidade. Assim, cada bloco diagonal forma uma 
matriz $(2 j+1) \times(2 j+1)$ tridiagonal:

$$
\begin{aligned}
h_{m} & =+\frac{N}{2}\left(\frac{N}{2}+\Gamma\right)+m(\Gamma-m), \\
g_{m} & =-\frac{1}{2}\left(m+\Gamma+\frac{N}{2}\right) \sqrt{j(j+1)-m(m+1)}, \\
\frac{\hat{\mathcal{H}}_{\mathrm{MF} j}}{\beta / N} & =\left(\begin{array}{llll}
h_{-j} & g_{-j} & \\
g_{-j} & h_{-j+1} & g_{-j+1} & \\
& g_{-j+1} & \ddots & g_{j-1} \\
& & g_{j-1} & h_{j}
\end{array}\right),
\end{aligned}
$$

que são matrizes de Jacobi. ${ }^{24}$ Do ponto de vista computacional, a diagonalização destas matrizes não exige muito recursos por ser esparsa e já na forma tridiagonal - normalmente utiliza-se rotações para atingir a forma tridiagonal. O maior bloco possui dimensão $N+1$ e também é o único setor que contempla os estados $\mid j=$ $N / 2, m= \pm N / 2\rangle$. Estes estados expressam a erradicação da doença $(m=-N / 2)$ ou a completa contaminação da população $(m=N / 2)$. Uma vez que os autovalores $\Lambda_{\mu}$ e autovetores $\left|\psi_{\mu}\right\rangle$ foram calculados para o operador $\hat{\mathcal{H}}_{\mathrm{MF}}$, podemos considerar os efeitos de perturbações sobre as conexões do grafo ou rede.

Como primeiro exemplo de perturbação, consideramos a remoção aleatória de arestas do grafo completo. A remoção ocorre com probabilidade $\delta p \ll 1$. Dessa maneira, o valor médio da matriz de adjacência perturbada, quando avaliada num ensemble, resulta em $A_{i j}=\left(1-\delta_{i j}\right)(1-\delta p)$. Claro, esse processo é equivalente à transformar a rede completa numa rede aleatória [106]. Como resultado, expressa-se a perturbação por meio do operador $\delta p \hat{V}=-\delta p \hat{\mathcal{H}}_{0}$ :

$$
\hat{V}=-\left(\frac{\beta}{2 N}\right)\left[\frac{N^{2}}{2}-2\left(\hat{J}^{z}\right)^{2}-\left(\hat{J}^{+} \hat{J}^{z}+\hat{J}^{z} \hat{J}^{-}\right)-N \hat{J}^{x}\right],
$$

que também satisfaz $\left[\hat{V}, \hat{J}^{2}\right]=0$.

Em relação aos estados estacionários do grafo completo, as correções de primeira ordem para o autovalor $\Lambda_{\mu}$ e para o autovetor $\left|\psi_{\mu}\right\rangle$ valem [41], respectivamente,

\footnotetext{
${ }^{24}$ Matrizes de Jacobi são geralmente associadas a polinômios ortogonais [126]. Na Eq. (3.77c), convém escrever $a_{m+j}=h_{m}$ e $b_{m+j}=g_{m}$. A combinação linear $|\psi\rangle=\sum_{n=0}^{2 j} c_{n}|j, n-j\rangle$ produz a seguinte relação de recorrência $a_{n} c_{n}+b_{n} c_{n+1}+b_{n-1} c_{n-1}=\lambda c_{n}$, onde $\lambda$ é o autovalor. Os coeficientes $c_{n}$ dependem do parâmetro que descreve o autovalor $\lambda \equiv \lambda(x), \operatorname{logo} c_{n}=c_{n}(x)$. Considere então a função geratriz $F(x, z)=\sum_{n=0}^{2 j} c_{n}(x) z^{n} / n !$. Se for possível eliminar os termos $a_{n}$ e $b_{n}$ em favor de derivadas nas variáveis $z$ e $x$, então obtém-se uma equação diferencial parcial para $F(x, z)$, cuja expansão em série de Taylor produz os coeficientes $c_{n}$. Como exemplo, considere o caso do polinômio de Hermite, cuja relação de recorrência é $H_{n+1}(x)+2 n H_{n-1}(x)=(2 x) H_{n}(x)$, com função geratriz $F(x, z)=\exp \left(2 x z-z^{2}\right)$. No caso da Eq. (3.77c), infelizmente, tal relação não foi obtida.
} 


$$
\begin{aligned}
& \Delta \Lambda^{(1)}=\delta p\left\langle\psi_{\mu}|\hat{V}| \psi_{\mu}\right\rangle, \\
& \Delta g_{\mu}^{(1)}=\delta p \sum_{\nu \neq \mu} \frac{\left\langle\psi_{\nu}|\hat{V}| \psi_{\mu}\right\rangle}{\left(\Lambda_{\mu}-\Lambda_{\nu}\right)} .
\end{aligned}
$$

Estas correções são então utilizadas para determinar a correção para as probabilidades das configurações,

$$
\Delta P_{\mu}^{(1)}=\sum_{\nu}\left\langle C_{\mu} \mid \psi_{\nu}\right\rangle \Delta g_{\nu}^{(1)}
$$

Alternativamente, nos regimes de equilíbrio ou máxima entropia, a substituição das correções da Eq. (3.79a) na Eq. (3.65) produz

$$
\sum_{\mu}\left[\left|g_{\mu}^{\prime}\right|^{2}\left(\Lambda_{\mu}+\Delta \Lambda_{\mu}^{(1)}\right)\right]=0
$$

Então, determina-se as novas soluções $g_{\mu}^{\prime}$, que carregam em si os efeitos da pertubação devido à primeira correção $\Delta \Lambda_{\mu}^{(1)}$.

Em nossa análise, toda a discussão acerca das correções para as probabilidades $P_{\mu}(t)$ envolvem apenas os autovalores e autovetores do operador simetrizado $\hat{\mathcal{H}}$, dada a perturbação $\hat{V}$. Isso evita o uso dos autovetores do operador assimétrico $\hat{H}$, uma característica geralmente desejável, e ao mesmo tempo permite o emprego de uma técnica de pertubação muito bem conhecida em processos epidêmicos.

\subsubsection{Redes periódicas}

Grafos completos são úteis quando o número de conexões típicas da rede são muito elevadas. Esse nem sempre é o caso. Existe um outro tipo de rede muito conveniente. Trata-se de redes invariantes por permutações cíclicas, as quais também são conhecidas por redes com invariância translacional. Essas redes têm como marco a decomposição espectral por meio de Transformadas de Fourier. Seus modos normais se ramificam em modos de longo e curto alcance, o que permite investigar o comportamento da disseminação de doenças e sua dependência com correlações de curto e longo alcance.

Considere a matriz de adjacência $A_{P}$ cujos elementos de matriz são $\left(A_{P}\right)_{k k^{\prime}}=$ $\left(\delta_{k, k^{\prime}+1}+\delta_{k, k^{\prime}-1}\right)$, com condições periódicas de contorno $V_{-1}=V_{N-1}$ e $V_{N}=V_{0}$. A rede descrita por essa matriz de adjacência cai dentro da categoria de redes regulares 
e periódicas, com período $N$. A partir das Eq. (3.74a) e (3.74b), determina-se o gerador temporal e simétrico $\hat{\mathcal{H}}_{P}$ correspondente ao processo epidêmico SIS para rede periódicas. Este operador atua como o operador não-perturbado para rede que derivam da rede periódica por meio de pequenas perturbações na distribuição de arestas.

Neste caso, as perturbações à rede regular periódica se dá por meio da adição de arestas entre vértices que não estejam previamente conectados. A adição destas arestas ocorre com probabilidade $\delta p$. Esse esquema de perturbação tenta reproduzir as propriedades e características mais relevantes das redes de mundo pequeno [108]. Com efeito, estas perturbações criam atalhos na disseminação de doenças entre os agentes $i$ e $j$ distantes, $|i-j|>1$. Assim como no caso das redes completas, se a pertubação for avaliada para um único grafo, a invariância por permutações cíclicas é perdida, ou seja, a expressão $N^{-1} \sum_{k}\left\langle\hat{O}_{k}\right\rangle=\left\langle\hat{O}_{1}\right\rangle$ não vale. Esse problema é contornado se considerarmos um ensemble de grafos, onde cada elemento do conjunto possui arestas adicionais (pertubação) distribuídas de acordo com a probabilidade $\delta p$.

Sob essa assunção, a matriz de adjacência média $\left\langle A_{i j}\right\rangle \equiv A_{i j}=\left(A_{P}\right)_{i j}+$ $\delta p\left(1-\delta_{i j}-\delta_{i, j+1}-\delta_{i, j-1}\right), \log \mathrm{A}=(1-\delta p) A_{P}+\delta p A_{\mathrm{MF}}$, onde $A_{\mathrm{MF}}$ é a matriz de adjacência da rede completa. Dessa maneira, o operador perturbado $\hat{\mathcal{H}}$ possui a contribuição da rede periódica somada à pertubação da rede de grafo completo,

$$
\hat{\mathcal{H}}=(1-\delta p) \hat{\mathcal{H}}_{P}+\delta p \hat{\mathcal{H}}_{\mathrm{MF}}
$$

Para $\delta p=0$, o gerador da Eq. (3.82) possui invariância por permutação cíclica, e adquire uma forma operatorial equivalente a de cadeias quânticas unidimensionais de spin $1 / 2$. Além disso, seu espectro pode ser classificado de acordo com o inteiro ${ }^{25} Q=0,1, \ldots, N-1$. Essas características sugerem o emprego de métodos matemáticos geralmente restritos ao estudo de sistemas exatamente integráveis ou fortemente correlacionados, como por exemplo, as variantes do ansatz de Bethe $[52,74]$. Com $\delta p \ll 1, \hat{\mathcal{H}}_{P}$ torna-se o operador não-perturbado e $\hat{\mathcal{H}}_{\mathrm{MF}}$ representa a pertubação. Já o caso de $1-\delta p \ll 1$ ramifica o problema em blocos disjuntos, identificados pelo momento angular total $j$, e transforma $\hat{\mathcal{H}}_{P}$ no operador perturbativo. Apresentamos outras técnicas no Apêndice 4

\footnotetext{
${ }^{25} \mathrm{Na}$ área de Física de Estado Sólido e de sistemas fortemente correlacionados, é comum atribui a interpretação de número de onda, ou momento, ao inteiro $Q$.
} 


\subsection{Outras aplicações epidemiológicas}

Na abordagem baseada em agentes para modelos epidêmicos, os agentes geralmente representam os indivíduos que podem eventualmente contrair a doença sob investigação. Essa é uma forma causal de se representar todos a cadeia de possíveis eventos que sucedem no processo saúde-doença. De certo modo, essa maneira examina o impacto de fatores biológicos no processo de disseminação na população. Contudo, agentes não precisam se restringir aos indivíduos se o foco do estudo for a disseminação de doenças. Essa dissociação entre a cadeia de infecção e o processo de disseminação permite a análise do problema sob uma visão mais generalista, com foco nas interações entre populações que formam uma determinada comunidade ao invés do processo biológico.

A fim de ilustrar este argumento, considere o seguinte cenário. Determinada comunidade é formada pelo conjunto de $L$ diferentes populações. Estas populações podem ser bairros, cidades ou até mesmo países, por exemplo, e possuem $N_{i}$ $(i=0,1, \ldots, L-1)$ indivíduos sob risco de determinada doença infecciosa. Para facilitar nossa discussão vamos impor $N_{i}=N$ para qualquer $i$. Os inter-relacionamentos entre as $L$ diferentes populações de uma comunidade são descritos pela matriz de adjacência $B$, cuja dimensão é $L \times L$ e traço nulo. A dificuldade desse cenário, que até aqui é muito semelhante ao do caso descrito nas seções anteriores, surge quando se considera a dinâmica de infecção, que somente é conhecida para indivíduos. Primeiramente, vejamos a dinâmica de infecção em única população. Uma vez entendido o mecanismo, podemos generalizar para a comunidade inteira.

Como sempre começamos pela definição do espaço de Hilbert para uma única população, contendo apenas indivíduos ou susceptíveis ou infectados. Como elemento descritor da $i$-ésima população, usamos o número de infectados $\left|n_{i}\right\rangle$ com $n_{i}=0,1, \ldots$ para caracterizar os diversos estados da população. Em princípio, o espaço de Hilbert limita-se a $N$ estados, porém deixamos $n_{i}$ inteiro e $n_{i} \in[0, \infty)$, ao invés de considerarmos o vínculo. Claro, o vínculo deve ser representado de alguma maneira e para isso define-se o projetor

$$
\hat{W}_{i}(\xi)=\frac{\mathrm{e}^{\xi\left(N-\hat{n}_{i}+1 / 2\right)}}{1+\mathrm{e}^{\xi\left(N-\hat{n}_{i}+1 / 2\right)}},
$$

em que $\xi \rightarrow \infty$. Se $n \leqslant N$, o argumento da exponencial torna-se positivo e, 
devido ao fator $\xi$, de elevado valor. Consequentemente, o projetor tende ao operador identidade; caso contrário, a exponencial torna-se o operador nulo. Assim, o projetor $\hat{W}_{i}(\xi)$ reproduz o vínculo e podemos trabalhar com todos os valores de $n_{i} \geqslant 0$ inteiros. Logo, o vetor probabilidade é

$$
|P(t)\rangle=W_{i}(\xi) \sum_{n_{i}=0} P_{n_{i}}(t)\left|n_{i}\right\rangle
$$

Para determinar a matriz de transição, convém começar pelo modelo SI, isto é, sem cura e apenas dois estados. Ao contrário da Eq. (3.32), onde começamos pelo mecanismo de infecção - que era conhecido - agora partimos do cálculo de permanecer na mesma configuração. Nesse ponto, assume-se a hipótese de mistura aleatória para a população, em conjunção com a correção de Pastor-Satorras e Vespignani [89], de maneira que o termo diagonal de $\hat{T}_{i}$ produz

$$
\left\langle n_{i}\left|\hat{T}_{i}\right| n_{i}\right\rangle=1-\frac{\beta}{N} \frac{\left\langle\kappa_{i}^{2}\right\rangle}{\left\langle\kappa_{i}\right\rangle}\left(N-n_{i}\right) n_{i}
$$

onde $\left\langle\kappa_{i}\right\rangle$ e $\left\langle\kappa_{i}^{2}\right\rangle$ descrevem o grau médio e segundo momento do grau da $i$-ésima população. Para simplificar a notação, define-se $\beta_{i}=\beta\left\langle\kappa_{i}^{2}\right\rangle /\left\langle\kappa_{i}\right\rangle$.

De acordo, a única transição fundamental possível descreve o aumento do número de infectados por uma unidade. No entanto, para que a soma dos elementos de matriz de $\hat{T}_{i}$ numa dada coluna seja unitária, devido à conservação da probabilidade total, temos a seguinte expressão:

$$
\left\langle n_{i}+1\left|\hat{T}_{i}\right| n_{i}\right\rangle=\frac{\beta_{i}}{N}\left\langle n_{i}+1\left|\hat{\mathcal{A}}_{i}^{\dagger} \hat{n}_{i}\left(N-\hat{n}_{i}\right)\right| n_{i}\right\rangle .
$$

Aqui, o operador $\hat{\mathcal{A}}_{i}^{\dagger}=\hat{a}_{i}^{\dagger}(\hat{n}+1)^{-1 / 2}$ reproduz a ação do operador levantamento $\hat{a}_{i}^{\dagger}$ do oscilador harmônico quântico, exceto pelo fator $\sqrt{n_{i}+1}$, isto é, $\hat{\mathcal{A}}_{i}^{\dagger}\left|n_{i}\right\rangle=\left|n_{i}+1\right\rangle$. Note que esse elemento de matriz é fundamentalmente diferente daquele descrito na Eq. (3.32), devido ao termo $\left(N-\hat{n}_{i}\right)$. No entanto, esse termo é o único que conserva a probabilidade nas colunas de $\hat{T}$.

A contribuição de ambas os termos diagonais e não-diagonais, em conjunto com o vínculo, resultam na matriz de transição

$$
\hat{T}_{i}=\hat{W}_{i}(\xi)\left[\mathbb{1}-\frac{\beta_{i}}{N}\left(\mathbb{1}-\hat{\mathcal{A}}_{i}^{\dagger}\right)\left(N-\hat{n}_{i}\right) \hat{n}_{i}\right] \hat{W}_{i}(\xi) .
$$

A generalização para incluir eventos de cura segue o mesmo raciocínio, produzindo 
a matriz de transição correspondente ao modelo SIS:

$$
\hat{T}_{i}=\hat{W}_{i}(\xi)\left[\mathbb{1}-\frac{\beta_{i}}{N}\left(\mathbb{1}-\hat{\mathcal{A}}_{i}^{\dagger}\right)\left(N-\hat{n}_{i}\right) \hat{n}_{i}-\gamma\left(\mathbb{1}-\hat{\mathcal{A}}_{i}\right) \hat{n}_{i}\right] \hat{W}_{i}(\xi),
$$

$\operatorname{com} \hat{\mathcal{A}}_{i}=\left(\hat{n}_{i}+1\right)^{-1 / 2} \hat{a}_{i}$.

O primeiro teste para a Eq. (3.88) é a equação que descreve o número médio de infectados da $i$-ésima população,

$$
\frac{d}{d t}\left\langle n_{i}(t)\right\rangle=\sum_{m_{i}=0}\left\langle m_{i}\left|\hat{n}_{i}\right| P(t)\right\rangle=\sum_{n_{i}=0}^{N} P_{n_{i}}(t)\left[\frac{\beta_{i}}{N}\left(N-n_{i}\right) n_{i}-\gamma n_{i}\right] .
$$

Se desprezarmos a variância, $\left\langle n_{i}^{2}\right\rangle=\left\langle n_{i}\right\rangle^{2}$, então reobtém-se a equação compartimental com a correção de Pastor-Satorras e Vespignani.

Com essas informações em mãos, a inferência sobre a matriz de transição de uma comunidade é simples. Em primeiro lugar, a base do espaço de Hilbert deve contemplar as $L$ populações, com seus respectivos número de infectados, $\left|n_{0}, n_{1}, \cdots, n_{L-1}\right\rangle$. Ademais, os projetores $\hat{W}_{i}(\xi)$ comutam entre si, de maneira que podemos representá-los por

$$
\hat{W}(\xi) \equiv \prod_{k=0}^{L-1} \frac{\mathrm{e}^{\xi\left(N-\hat{n}_{i_{k}}+1 / 2\right)}}{1+\mathrm{e}^{\xi\left(N-\hat{n}_{i_{k}}+1 / 2\right)}} .
$$

Com espaço de Hilbert e o projetor $W(\xi)$ apropriadamente definidos, podemos então nos concentrar na dinâmica efetiva do modelo SIS para comunidades. A Eq. (3.88) serve como inspiração para a formulação da matriz de transição, a partir da qual experimenta-se o ansatz

$$
\hat{T}=\hat{W}(\xi)\left[\mathbb{1}-\frac{\beta}{N} \sum_{i j=0}^{L-1} \frac{B_{i j}^{\prime}}{L}\left(\mathbb{1}-\hat{\mathcal{A}}_{i}^{\dagger}\right)\left(N-\hat{n}_{i}\right) \hat{n}_{j}-\gamma \sum_{i}^{L-1}\left(\mathbb{1}-\hat{\mathcal{A}}_{i}\right) \hat{n}_{i}\right] \hat{W}(\xi) .
$$

Os valores $B_{i j}^{\prime}$ correspondem aos elementos de matriz da matriz de adjacência efetiva $B^{\prime} \equiv B^{\prime}(B)$.

Ora, mas qual a razão para se complicar o problema por meio da introdução de uma matriz a mais? O adjetivo "efetivo" para $B^{\prime}$ se deve à maneira como as conexões de $B$ são determinadas. Mais especificamente, a relevância das conexões entre as populações, dentro da escala de tempo empregada no cálculo da taxa de infecção $\beta$. Isto se dá devido à maneira como as conexões são modeladas. Se elas reproduzem algum algum tipo de fluxo, de produtos ou pessoas, mesmo que 
$B_{i j}=0$ a propagação da doença pode ocorrer por rotas alternativas que conectam os vértices $j$ e $i$, devido ao intervalo de tempo $\delta t$ elevado - tipicamente dias nas escalas epidemiológicas. Isso equivale a uma conexão efetiva entre os vértices $i$ e $j$.

Levando em conta as considerações acima, os elementos de matriz $B_{i j}^{\prime}$ devem conter contribuição do elemento $B_{i j}$ correspondente, além da contribuição média dos caminhos formados por apenas um vértice intermediário:

$$
\frac{B_{i j}^{\prime}}{L}=\frac{1}{z}\left(\frac{B_{i j}}{L}+\frac{1}{L-1} \sum_{k=0}^{L-1} \frac{B_{i k}}{L} \frac{B_{k j}}{L}\right),
$$

onde $z$ é um fator de normalização a ser determinado e $i \neq j$. O fator $L^{-1}$ é conveniente pois sabe-se que $\sum_{i} B_{i j} \leqslant L$, de modo que rotas com múltiplos vértices carregam peso menor do que a conexão direta $B_{i j} .{ }^{26} \mathrm{O}$ valor de $z$ pode ser determinado a partir do grafo completo e seu análogo efetivo. Esse caso é singular pois ambas as matrizes de adjacência correspondentes devem ser idênticas e, portanto, $z=1+L^{-1}$. Por fim, impomos que as componentes diagonais são

$$
B_{i i}=\frac{\left\langle\kappa_{i}^{2}\right\rangle}{\left\langle\kappa_{i}\right\rangle}
$$

a fim de reobter as Eq. (3.88). ${ }^{27}$

Todas as técnicas discutidas ao longo deste capítulo também se aplicam à Eq. (3.91), pois trata-se de um processo Markoviano. Mas há um ingrediente extra que facilita toda a discussão em torno do processo epidêmico: o espaço de Hilbert. Por considerar apenas uma única variável descritiva (número de infectados) para cada uma das $L$ populações, a complexidade do problema é reduzida consideravelmente. De fato, se considerarmos todos os indivíduos, a dimensão do espaço de Hilbert seria $2^{N L}$, enquanto que essa versão semi-compartimental contempla um espaço vetorial de dimensão $N^{L}$. De certo modo, a heterogeneidade local - os detalhes locais da população - no processo de infecção são desprezados frente à heterogeneidade das diferentes populações. Por esse motivo, denominamos esse tipo de modelagem por abordagem semi-compartimental.

Além dos métodos de muitos corpos (DFT e campo médio) e perturbativos, nutrimos interesse especial nessa modelagem no que diz respeito às transformações

\footnotetext{
${ }^{26}$ Vale a pena mencionar que o termo $(1 / L) \sum_{i k} B_{i k} B_{k i}=(1 / L) \operatorname{Tr} B^{2}=\left\langle\kappa^{2}\right\rangle$ possui certa semelhança com o termo $\left(1 / L^{2}\right) \sum_{k} B_{i k} B_{k j}$.

${ }^{27}$ Sob a condição $B^{\prime}=B$ é fácil verificar que o operador da Eq. (3.91) é equivalente ao operador da Eq. (3.32) para $N=1$.
} 
de escala. Quais os impactos esperados ao se mudar o nível de detalhamento da modelagem para a dinâmica estocástica? Em sistemas críticos, dominados por efeitos coletivos [16], flutuações de determinadas observáveis são invariantes por transformações de escala, decorrência da correlação de longo alcance entre os componentes do sistema. Esse comportamento é caracterizado por expoentes, denominados expoentes críticos. Em geral, o comportamento crítico é investigado segundo as ideias de Wilson do Grupo de Renormalização [82]. Comparando-se as Eqs. (3.32) e (3.91), observa-se que ambos geradores relacionam-se segundo o Grupo de Renormalização. A conexão precisa dessas transformações, não só para o modelo SIS mas para outros modelos como SIRS e SEIRS, pode contribuir significativamente na compreensão na formação de epidemias e/ou pandemias.

\subsection{Conclusão}

A conexão adequada entre a sucessão de eventos biológicos presentes durante o desenvolvimento de determinada doença e seu processo de disseminação ainda se apresenta como desafio na modelagem epidemiológica. A abordagem compartimental, mais tradicional e com bons resultados em alguns casos, despreza a influência da diversidade presente numa população. As simplificações decorrentes produzem sistemas de equações diferenciais, cuja solução descreve o processo de disseminação da doença entre os membros da população. Apesar de seus avanços, especialmente quando se adiciona correções oriundas de sistemas complexos, a abordagem falha quando as particularidades e detalhes da população são críticos no processo epidêmico.

A inclusão de tais detalhes tem papel diferenciado e relevante na abordagem baseada em agentes, que trata cada indivíduo de maneira independente. A consideração de tais detalhes e a natureza intrinsecamente estocástica do problema permite o esclarecimento de alguns resultados contraintuitivos da abordagem compartimental, bem como o modelamento adequado de doenças emergentes. Contudo, o excesso de informação disponível restringe o poder de previsibilidade dessa metodologia à pequenas populações.

Nossa contribuição nessa área de grande interesse social, devido ao seu impacto na governança e planejamento da Saúde Pública, simplifica substancialmente 
a análise espectral das abordagens baseadas em agentes. Em particular, o desenvolvimento da Eq. (3.65) abre uma nova forma de se estudar processos epidêmicos, especialmente a região de máxima entropia durante o regime transiente. Ainda restam diversas questões a serem resolvidas, tanto do ponto de vista computacional e teórico. Essas tarefas, entretanto, exigem diversos novos estudos. De particular importância serão aqueles que irão testar a compatibilidade da Eq. (3.65) com dados experimentais, sujeitos a todo tipo de imprecisões. Se porventura nossa contribuição sobreviver ao árduo teste científico experimental, então nossa ferramenta com certeza produzirá efeitos observáveis na caracterização de epidemias.

Apesar de ter sido deduzida somente para processos Markovianos, a Eq. (3.65) também pode ser utilizada em processos com memória, mediante introdução de graus de liberdade adicionais. Essa temática muito interessante foge muito ao tema desta tese mas, por completeza, apresentamos alguns comentários e resultados no Apêndice 5. Em relação à teoria e ao desenvolvimento apresentados neste capítulo, apresentamos e discutimos duas ramificações que podem ser examinadas como linhas de pesquisa num futuro próximo.

A primeira ramificação consiste em fortalecer a analogia dos operadores da abordagem baseada em agentes com os operadores e técnicas da Teoria Quântica. Dentre elas, as propriedades derivadas de simetrias, bem como as técnicas e aproximações de muitos corpos, sejam elas numéricas ou puramente analíticas. Há, portanto, necessidade de se definir desde propriedades simples como relações de comutação entre operadores, como aquelas mais complexas que caracterizam quasipartículas e suas respectivas distribuições. Como exemplo, mencionamos algo corriqueiro para os versados na Mecânica Quântica mas explorado apenas por meios computacionais em Epidemiologia. Trata-se de fontes puntuais. Os operadores de permutação cíclica, conforme explicado no texto e no Apêndice 2, não comutam com fontes de doença puntuais. Grosso modo, temos o comutador $[\hat{P}, \hat{X}]=$ cte. Estudos mais aprofundados sobre a natureza e impacto dessas propriedades certamente serão examinados futuramente, num momento adequado. 


\title{
4
}

\section{Simetrias em cavidades ópticas}

\author{
Sem quaisquer propriedades da simetria subjacente, o trabalho de \\ provar resultados interessantes torna-se extremamente desagradável. \\ - Donald Knuth
}

Dentre as muitas áreas do conhecimento humano, o Eletromagnetismo apresenta-se indiscutivelmente como um de seus pilares mais relevantes. A fundamentação e domínio da teoria eletromagnética clássica foi o berço do intenso avanço tecnológico experimentado no século passado; do desenvolvimento da teoria da Relatividade, etc. Tudo isso baseado no simples conceito de carga elétrica e campos, vide força de Coulomb, cuja dedução é o epítome do processo dedutivo cargas opostas se atraem, similares se repelem, sempre na direção que as une e decai com o inverso da distância. De fato, é impossível enumerar todas as contribuições do Eletromagnetismo. A compatibilização da teoria eletromagnética com a teoria quântica tomou forma inicialmente sob os auspícios de Dirac e Fermi [127]. Um dos problemas dessa teoria foi o surgimento de divergências no tratamento perturbativo. Um primeiro remédio foi desenvolvido por Bethe [128] para explicar o Lamb shift, que descreve um deslocamento dos níveis de energia atômico devido à interação dos elétrons com o campo de radiação. ${ }^{1}$ Nele, Bethe dá início ao processo de renormalização de carga e massa, posteriormente conhecido como a marca registrada da Eletrodinâmica Quântica (QED).

Aqui, não pretendemos explorar os nuances da QED em sua totalidade. Ao

\footnotetext{
${ }^{1}$ Reza a lenda que a dedução da correção do Lamb Shift foi desenvolvida ao longo de uma viagem de trem, para atender a uma conferência. O artigo científico de fato possui em torno de duas páginas e é bastante acessível. Dentre suas outras notáveis contribuições também se destaca o resgate sua mãe da Alemanha nazista e um prêmio Nobel com a publicação de um único artigo científico.
} 
contrário, vamos nos ater a um problema simples, mas muito interessante, que trata da interação entre campos eletromagnéticos radiativos e átomos (matéria), confinados numa cavidade óptica. Mais especificamente, queremos estudar aspectos físicos que envolvam simetrias e suas relações com a teoria do momento angular.

Uma pergunta natural que podemos realizar acerca desse estudo trata da conexão entre entre um problema de interação radiação-matéria com simetrias e momento angular. A motivação decorre da observação devido a Dicke [24] que o campo eletromagnético confinado na cavidade é compartilhado por todos os demais átomos, dando origem a fenômenos coletivos. Um destes efeitos é a amplificação da emissão espontânea e coerente de fótons pelos átomos, mais conhecido como superradiância. No que segue, estudamos dois sistemas. O primeiro contém $N$ átomos ultrafrios de dois níveis que interagem com um único modo de radiação. No segundo sistema, consideramos duas espécies distintas de átomos. A simetria $U(1)$, que relacionamos com a conservação da carga elétrica, sugere estudos aprofundados sobre o conceito de quasipartículas e conservação do momento angular. A partir dessas investigações, obtemos uma interpretação para a contribuição anti-girante como uma interação spin-órbita, cuja ação habilita fenômenos de interferência entre átomos ultrafrios distintos.

\subsection{Modelo de Dicke}

A interferência de ondas eletromagnéticas é um dos aspectos mais marcantes do comportamento ondulatório e, a partir das ideias de de Broglie, também é compartilhado pelo particulado nas escalas subatômicas. Naturalmente há certas diferenças em se lidar com interferências oriundas de fontes ópticas daquelas de origem particulada. Neste último caso, as partículas eventualmente podem interagir entre si ou ser manipuladas por meio de campos externos. Eventualmente, essa propriedade permite ao experimentador induzir ou sintonizar interferências. Experimentos nessa direção tem sido bastante explorados por meio de materiais nanoestruturados como pontos quânticos [129-131], nanopartículas metálicas [132], e até mesmo com nanoestruturas mais complexas $[133,134]$. As interferências de natureza quântica também são vitais para a teoria de informação quântica, como discutem as Refs. [135, 136], bem como no estudo de átomos ultrafrios [137, 138]. 
Todas as referências citadas no parágrafo acima compartilham uma fase superradiante, fenômeno esse intrinsecamente ligado à interferência quântica. Superradiância nada mais é do que um fenômeno coletivo no qual $N$ átomos confinados numa cavidade óptica emitem luz coerente de maneira espontânea. A superradiância foi prevista por Dicke [24], em 1954, cujo argumento observa que o campo eletromagnético presente dentro da cavidade óptica é o mesmo para todos os átomos, introduzindo correlações entre os átomos [139-141]. Neste regime, a emissão espontânea não ocorre como $N$ eventos independentes, mesmo para $N \sim o(1)$, como comprovado por experimentos cuidadosos recentes [142]. Para uma cavidade com $N$ átomos em seu estado excitado, este comportamento coletivo decorre a partir de um primeiro decaimento atômico. A radiação resultante induz os outros átomos a decaírem também, promovendo uma resposta positiva de grande amplitude [131], ou seja, um pulso com intensidade proporcional a $N^{2}[140,143]$. Como consequência, o tempo de decaimento $\tau_{\mathrm{sr}}=\tau_{\mathrm{sp}} / N$ de átomos no regime superradiante é $N$ vezes menor que o tempo de decaimento por emissão espontânea individual $\tau_{\mathrm{sp}}$.

No tratamento da teoria de interação radiação-matéria, costuma-se introduzir a aproximação de ondas girantes (RWA, do inglês rotating wave approximation). Os operadores correspondentes às baixas frequências são ditos girantes, enquanto os operadores de alta frequência produzem as contribuições anti-girantes. No picture de Interação [77], esta aproximação despreza contribuições de alta frequência em favor daquelas de baixa frequência, propiciando uma simplificação extensiva ao problema [77]. Isto também permite a interpretação intuitiva para o processo de absorção e emissão [26], no qual a transição atômica de um nível de maior energia para um de menor energia sempre ocorre acompanhada pela emissão de um fóton, e vice versa. As variedades de operadores Hamiltoniano resultantes são geralmente postas conforme apresentadas nas Refs. [25, 60], que são bem conhecidos e apresentam grande difusão acadêmica e tecnológica.

Porém, apesar do grande sucesso da RWA, existem evidência experimentais e indícios teóricos que sugerem que os termos anti-girantes não podem ser descartados [144]. Como demonstrado na Ref. [145], o emaranhamento de qubits atravessa uma descontinuidade devido aos operadores anti-girantes. Sob a RWA, tal discontinuidade não é observada. Além da RWA, existe uma solução exata no limite 
termodinâmico utilizando as transformações de Holstein-Primakoff [146, 147]. Esta solução permite a identificação de transições de fase quântica, incluindo a transição de emissão espontânea convencional para o regime superradiante. Para $N$ finito, apenas soluções numéricas abrangem todos os valores de acoplamento [148]. Ao longo dos anos, técnicas analíticas tem sido introduzidas a fim de se mitigar a RWA mas manter sua interpretação conveniente de absorção e emissão de partículas (fótons) $[136,149-152]$.

Curiosamente, apesar de todo arcabouço teórico e experimental no estudo de modelos de cavidades, a conservação de partículas e de momento angular são geralmente tópicos desprezados. Ambos levam a leis de conservação muito importantes e a interpretações um pouco diferentes daquelas de Emary e Brandes [146], quanto à natureza da quasipartícula. Para isso, faz-se mister determinar as simetrias do problema de interação entre um único modo radiativo, com frequência angular $\omega$, e $N$ átomos de dois níveis, cujas energias são $\pm \hbar \Omega / 2$, presos num cavidade nãodissipativa. A motivação é bastante simples. A interação radiação-matéria é conservativa e depende apenas de produtos dois-a-dois de operadores [153], afinal, deriva diretamente de um potencial conservativo e todo o processo se desenrola confinado numa cavidade não-dissipativa. Apesar disso, ela não exibe conservação nem do número de fótons ou de excitação atômica, fora da RWA. A primeira etapa, portanto, é reconciliar a ideia de conservação de energia com modos coletivos ou individuais que absorvem ou emitem fótons. Para esta finalidade, podemos nos valer da conservação da carga elétrica, que gera a simetria $U(1)$. Como resultado, espera-se invariância por transformações de gauge de primeiro tipo [42, 77]. Vejamos abaixo como essa simetria se desenvolve no modelo de Dicke para a interação radiação-matéria [24].

A Figura 4.1 ilustra todos os processos físicos descritos pelo modelo de Dicke, cujo Hamiltoniano é

$$
\hat{H}=\hbar \omega \hat{a}^{\dagger} \hat{a}+\Omega \hat{J}^{z}+\lambda\left(\hat{a}+\hat{a}^{\dagger}\right)\left(\hat{J}^{-}+\hat{J}^{+}\right)
$$

onde $\hat{a}$ e $\hat{a}^{\dagger}$ são, respectivamente, os operadores de destruição e criação de modos radiativos bosônicos, $\left[\hat{a}, \hat{a}^{\dagger}\right]=1$; os operadores coletivos $\hat{J}^{\alpha}=\sum_{k=1}^{N} \hat{J}_{k}^{\alpha}(\alpha=z, \pm)$ são obtidos mediante adição dos $N$ operadores correspondentes a cada átomo $\hat{J}_{k}^{\alpha} ;$ o operador de nível eletrônico $\hat{J}_{k}^{z}$ determina o estado de excitação do $k$-ésimo átomo, enquanto $\hat{J}_{k}^{ \pm}$são os operadores de levantamento e abaixamento para o $k$-ésimo 

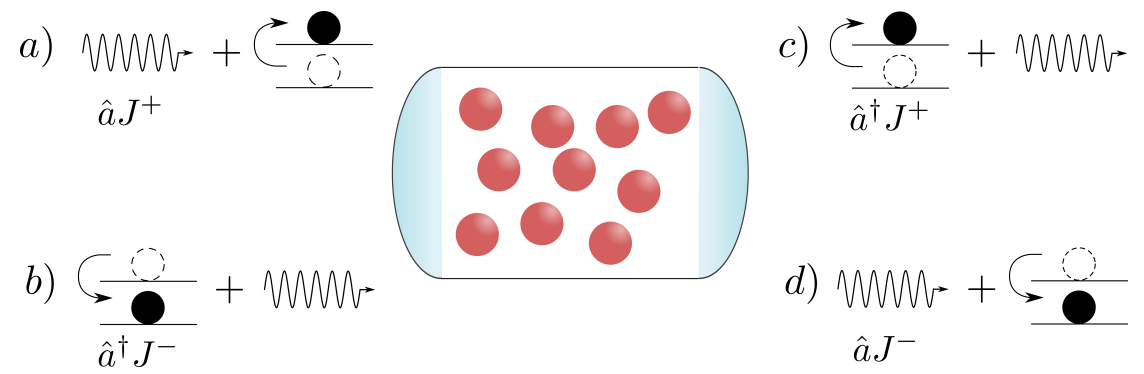

Figura 4.1: Interação radiação-matéria no modelo de Dicke. Um estado com $n$ fótons e $M^{z}+N / 2$ átomos excitados é submetido a quatro diferentes transições: a) excitação atômica com absorção de fóton; b) decaimento atômico com emissão de fóton; c) excitação atômica com emissão de fóton; d) decaimento atômico e absorção de fóton. Na RWA, apenas os processos a) e b) são relevantes.

átomo. Como usual, os operadores atômicos $\hat{J}_{k}^{ \pm, z}$ formam uma álgebra de Lie $\left[J_{m}^{+}, J_{n}^{-}\right]=2 \hbar J_{m}^{z} \delta_{m n}$ e $\left[J_{m}^{z}, J_{n}^{ \pm}\right]= \pm \hbar J_{m}^{ \pm} \delta_{m n}$. Como de costume, os operadores de matéria e de radiação comutam entre si.

A interação radiação-matéria é descrita pela parcela da Eq. (4.1) acompanhada pelo acoplamento $\lambda^{2}$ A interação, portanto, é Hermitiana e possui duas contribuições distintas, a saber, girante e anti-girantes, que são respectivamente

$$
\begin{aligned}
& \hat{a}^{\dagger} \hat{J}^{-}+\hat{J}^{+} \hat{a}, \\
& \hat{a}^{\dagger} \hat{J}^{+}+\hat{J}^{-} \hat{a} .
\end{aligned}
$$

A contribuição girante (4.2a) tem um forte apelo com os processos de emissão e absorção de fótons por férmion: absorve-se um fóton e, em contrapartida, excita-se o átomo. De fato, esse é o comportamento típico para gases descorrelacionados. Em geral, essa contribuição perturbativa apresenta-se como termo dominante seja no picture de interação ou em análises semiclássicas. A RWA assume que apenas os operadores girantes produzem efeitos relevantes para o problema.

A contribuição anti-girante (4.2b), assim como a contribuição girante, também é Hermitiana. Apesar disso, os operadores anti-girantes são geralmente classificados como operadores que não conservam a energia pois quebram o paradigma de emissão e absorção de fótons por férmions. Assim como ocorre em matéria condensada, parte do problema surge pois assume-se que os operadores $\hat{J}^{z, \pm}$ descrevem férmions. De acordo com a discussão do Capítulo 2, estes operadores tem um caráter dual: são tanto bosônicos, pois operadores de átomos distintos comutam, como

\footnotetext{
${ }^{2}$ Vale a pena salientar que $|\lambda|^{2}$ é proporcional a taxa de transições experimentada por $N$ átomos sob condições controladas. Eventuais dependências de $\lambda$ com parâmetros intensivos, como temperatura e pressão, são desprezados neste estudo.
} 
fermiônicos, $\left\{J_{k}^{+}, J_{k}^{-}\right\}=\hbar^{2}$. Em altas temperaturas ou baixas densidade atômica, espera-se pouca superposição das funções de onda de maneira que o caráter dual não é importante. Já no caso de baixas temperaturas e altas densidades, espera-se que efeitos associados à natureza fermiônica ou bosônica tenha papel relevante. São exatamente esses casos onde a RWA não se aplica [154].

No que segue, adotamos apenas fótons na frequência de ressonância do átomo, $\omega=\Omega$ para $N$ finito. Apesar de não ser muito realista, assumimos que a cavidade óptica é ideal e não dissipa energia. Fótons emitidos por radiação de corpo negro pela cavidade também são desprezados.

\subsection{Efeitos de $N$ finito}

Em geral, propriedades macroscópicas de sistemas atômicos são expressas pelas densidades atômicas e de fótons, $N / V$ e $\langle n\rangle / V$, respectivamente. O limite termodinâmico é atingido quando ambas as razões possuem valores bem definidos conforme o volume $V \rightarrow \infty$. Neste regime, quantidades extensivas escalam tanto com o número médio de fótons, quanto com o número médio de átomos no estado excitado $\left\langle J^{z} / \hbar+N / 2\right\rangle$ aprisionados na cavidade, como prega as linhas gerais da Termodinâmica. Porém, podemos aprender muito sobre as propriedades do sistema se considerarmos o regime finito. Muitas das hipóteses contidas nos estudos de Informação Quântica e Nanomateriais se realizam exatamente neste regime diferenciado. Devido à finitude das variáveis relevantes, efeitos não-triviais são observados, que se apresentam como correções não-extensivas e com bom potencial para aplicações tecnológicas. Este regime também permite um primeiro exame mais visceral sobre as simetrias do problema.

Para isto, convém começar o problema de uma situação com muitos fótons e, então, observar como o sistema se comporta com a redução gradual de $\langle n\rangle$. Para uma densidade muito alta de fótons, o campo de radiação dentro da cavidade permanece praticamente inalterado no limite $N / V \rightarrow 0$. Como resultado, podemos descrever o campo de radiação de uma maneira razoavelmente simples por meio de estados coerentes $|\gamma\rangle$, tal que $\hat{a}|\gamma\rangle=\gamma|\gamma\rangle$. Sob estas condições, o Hamiltoniano da Eq. (4.1) assume uma forma muito mais simples,

$$
\hat{H}_{\mathrm{ch}}=\hbar \omega|\gamma|^{2}+\Omega \hat{J}^{z}+2 \lambda \operatorname{Re}(\gamma)\left(\hat{J}^{-}+\hat{J}^{+}\right) .
$$


Logo, o operador $\hat{H}_{\text {ch }}$ é apenas a combinação de $N$ operadores disjuntos, cada um com autoenergias $E_{ \pm}= \pm(\hbar \Omega / 2) \sqrt{1+16 \operatorname{Re}(\gamma)^{2}(\lambda / \Omega)^{2}}$. Assim, o espectro de energia total é obtido mediante combinações das autoenergias $E_{ \pm}$para os $N$ átomos. Essa informação pode ser utilizada, por exemplo, para se calcular a função de partição do sistema, $Z(T)$, para a temperatura $T$. Ademais, essa discussão é condizente com a hipótese inicial de que o número médio de fótons, $\langle n\rangle / V=|\gamma|^{2} / V$, permanece inalterado. Quanto a parte atômica, o Hamiltoniano da Eq. (4.3) descreve, grosso modo, o modelo de spins não-interagentes sob ação de um campo transverso.

O outro caso, quando $\langle n\rangle / V \rightarrow 0$, produz efeitos muito mais interessantes. Para investigá-lo, porém, precisamos especificar como os fótons são removidos da cavidade, a partir do caso anterior. Por simplicidade, podemos considerar um procedimento no qual um fóton é removido por vez da cavidade, mantida a baixas temperaturas $T \rightarrow 0$. Se o procedimento é iterado, a assunção que o campo de radiação é descrito por estados coerentes não se sustenta. De fato, podemos introduzir um corte explícito $l$ no número de fótons, como feito numericamente na Ref. [61]. Este procedimento equivale a truncar o espaço de Fock correspondente, permitindo o estudo de efeitos finitos para baixas energias. Estados de altas energias contém um número maior de fótons e, portanto, não podem ser investigados após o truncamento. Em luz destas considerações, restringimos nossa análise aos estados de menor energia com ênfase ao estado fundamental.

A primeira análise examina numericamente o número médio de fótons no estado fundamental, como uma função de $l$ e $\lambda$, com $N$ e $\omega$ fixos. Para isso, implementamos um código computacional que diagonaliza o Hamiltoniano da Eq. (4.1), para $N \sim o\left(10^{1}\right)$, posto que o espaço de Fock correspondente cresce como $l \times 2^{N}$. Seja $\nu=0,1, \ldots, l \times 2^{N}-1$ o índice dos vetores da base do espaço de Fock, $\left|\phi_{\nu}\right\rangle$. Estes índices são melhor descritos pela fórmula $\nu=\alpha+\left(2^{N}\right)^{m}, \operatorname{com} \alpha=0,1, \ldots, 2^{N}-1$ e $m=0,1, \ldots, l-1$, que essencialmente quebra a enumeração do espaço vetorial em $l$ segmentos com $m$ fótons e $N$ átomos de dois níveis. Nesta base o estado fundamental possui a seguinte decomposição $\left|\psi_{0}\right\rangle=\sum_{\nu} c_{\nu}\left|\phi_{\nu}\right\rangle$. Por conta da segmentação ordenada do espaço de Fock, convém definir o índice médio do estado fundamental $\mu \equiv \sum_{\nu} \nu\left|c_{\nu}\right|^{2}$ e a respectiva variância $\sigma^{2}=\sum_{\nu}(\nu-\mu)^{2}\left|c_{\nu}\right|^{2}$. Em princípio, um aumento na densidade média de fótons também implica num aumento da média $\mu$. 


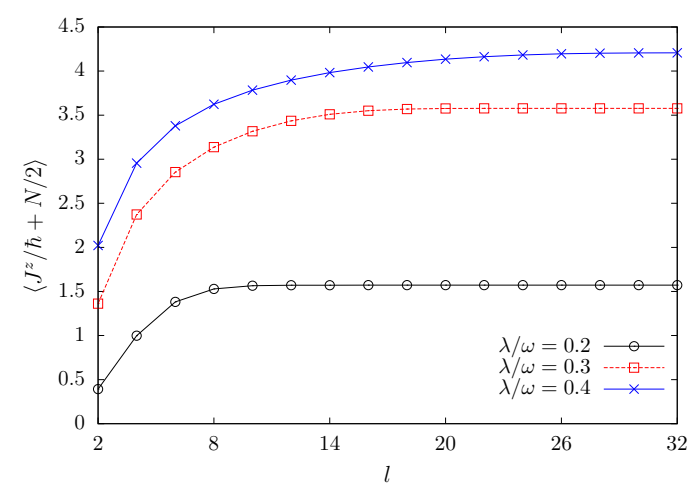

(a)

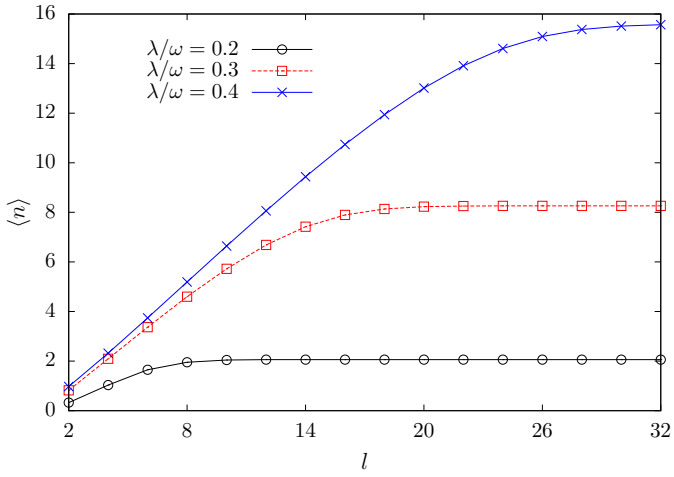

(b)

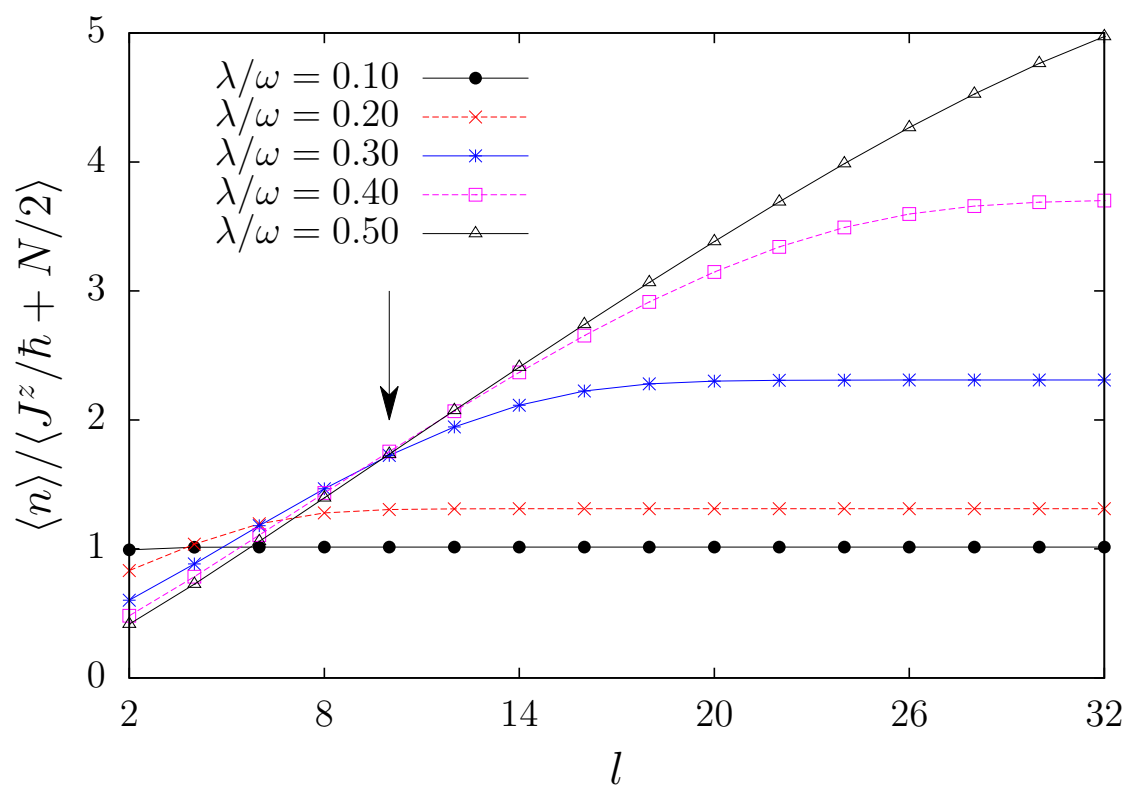

(c)

Figura 4.2: Efeitos de tamanho finito. a) o número médio de átomos excitados $\left\langle J^{z} / \hbar+N / 2\right\rangle$ e b) o número médio de átomos $\langle n\rangle$, ambos para o estado fundamental do modelo de Dicke, de acordo com o valor do corte $l$. Correções devido a $l$ finito são observáveis em ambos os casos até atingir um limiar $l_{c}(\lambda)$ para cada acoplamento $\lambda$. Em c), a flecha indica que a razão $r_{N}(\lambda, l)=$ $\langle n\rangle /\left\langle J^{z} / \hbar+N / 2\right\rangle$ desenvolve um ponto de cruzamento para $l=N$ para $\lambda>\lambda_{c}$.

Como o Hamiltoniano do problema possui invariância por permutações cíclicas quanto aos átomos, utilizamos a base cíclica conforme descrito no Apêndice 2, reduzindo substancialmente a complexidade computacional em troca da introdução de um número quântico adicional. Ao variar-se $l \operatorname{com} \lambda$ fixo, observa-se que existe um valor $l_{c}(\lambda)$, a partir do qual incrementos subsequentes de $l$ mantém $\langle n\rangle$ inalterado. Comportamento semelhante é observada para o número médio de átomos excitados, uma vez que os campos de radiação e matéria estão acoplados. A Figura 4.2 mostra os resultados numéricos para ambos $\left\langle J^{z} / \hbar+N / 2\right\rangle$ e $\langle n\rangle$ com $\lambda / \omega=m / 10$ 
$(m=2,3,4)$ e $N=10$.

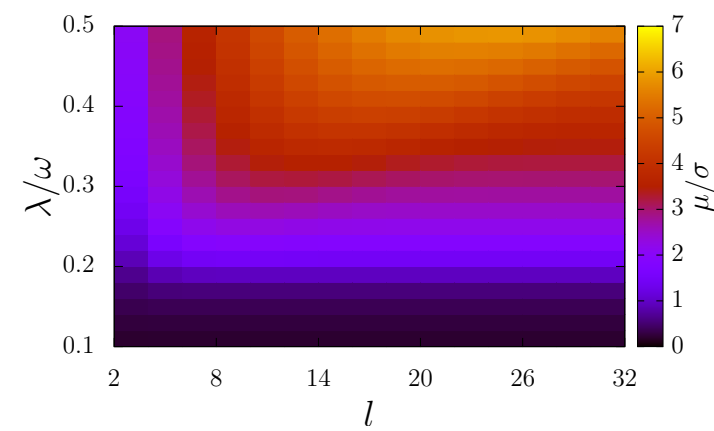

(a)

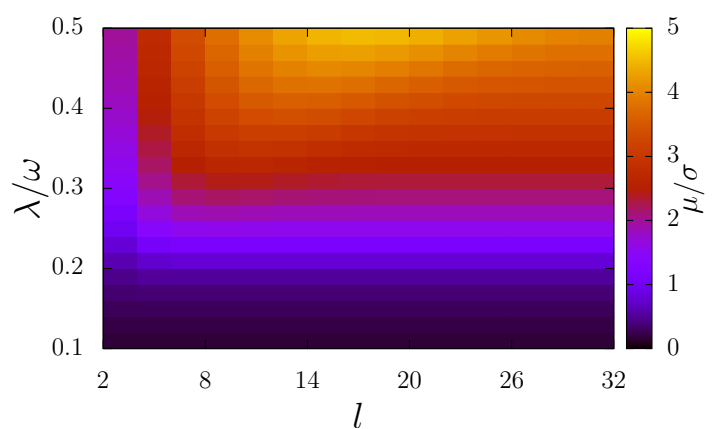

(b)

Figura 4.3: Relação sinal-ruído. a) A relação sinal-ruído para $N=10$. Para baixos valores de acoplamento $\lambda$, a energia do estado fundamental $E_{0}=-\hbar \Omega N / 2$ independe de $\langle n\rangle$ e o estado fundamental exibe forte resistência à correções de tamanho finito. Para $\lambda>\lambda_{c}, \mu / \sigma$ desenvolve um gradiente positivo com $l$ e $\lambda$. b) Comportamento semelhante é observado para $N=8$.

Para $l \gg N$, ambos número médio de fótons e estados excitados assumem valores constantes. Assim, a razão $r_{N}(\lambda, l)=\langle n\rangle /\left\langle J^{z} / \hbar+N / 2\right\rangle$ também adquire valor constante e serve para identificar o regime físico do sistema. No que segue, a análise centra-se ao redor do corte $l=N$, onde se espera a emergência de efeitos finitos já que o número máximo de fótons é igual ao número total de átomos. De acordo com nosso argumento anterior, correções devido ao número finito de fótons são esperadas com o aumento do valor do acoplamento $\lambda$. Nossos resultados numéricos indicam que existe um valor crítico $\lambda_{c}$, no plano $l=N$, a partir do qual $r_{N}\left(\lambda \geqslant \lambda_{c}, l=N\right) \equiv r_{c}(N)$, isto é, depende unicamente do número total de átomos como mostra a Figura. 4.2c). O acoplamento $\lambda_{c}$ marca, portanto, dois comportamentos distintos para o estado fundamental. No primeiro, com $\lambda<\lambda_{c}$, o estado fundamental é insensível à correções não-extensivas devido ao corte $l$. Esse efeito também pode ser observado por meio da relação sinal-ruído $\mu / \sigma$, ilustrado pela Figura 4.3. Já na região complementar, efeitos oriundos do corte são relevantes.

A fim de complementar nossa análise, convém utilizar o fator de Fano, $F_{N}(\lambda, l)=\sigma^{2} / \mu \equiv F$, mais conhecido como índice de dispersão em Estatística. A Figura 4.4 mostra que $F$ possui um máximo para $l \geqslant N$ para $\lambda=\lambda_{c}$. Além disso, $F$ é insensível a efeitos finitos desde que $l \geqslant N$ e $\lambda<\lambda_{c}$, em concordância com nossos resultados anteriores. Porém, ele também desenha três regiões em nosso espaço de parâmetros com diferentes características. Na primeira região, designada 
por $R_{1}$, o fator $F$ é uma função decrescente de $\lambda$, reflexo de correções devido ao corte $l$. Na segunda região, $R_{2}, F$ ainda decresce com $\lambda$ mas a uma taxa muito mais lenta do que em $R_{1}$, criando platôs para $l \gg N$. Logo, nessa região, o formato da distribuição dos coeficientes lineares do estado fundamental se mantém aproximadamente constante para diferentes valores de $\lambda$. Por fim, na região $R_{3}$, efeitos devido à finitude do sistema não são apreciáveis.

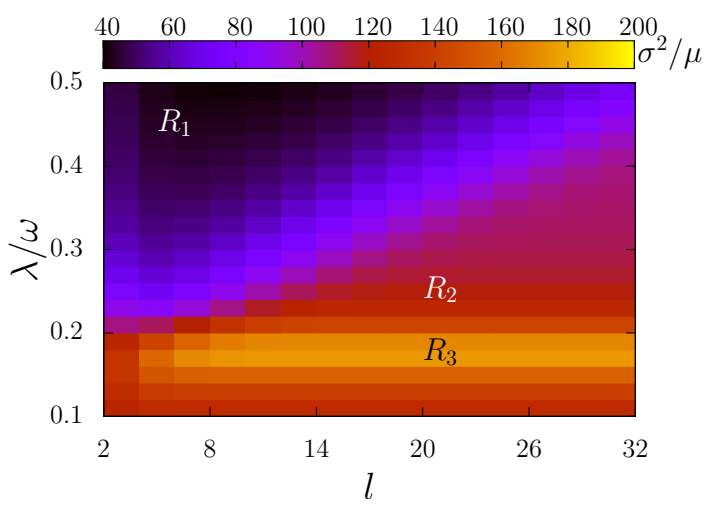

(a)

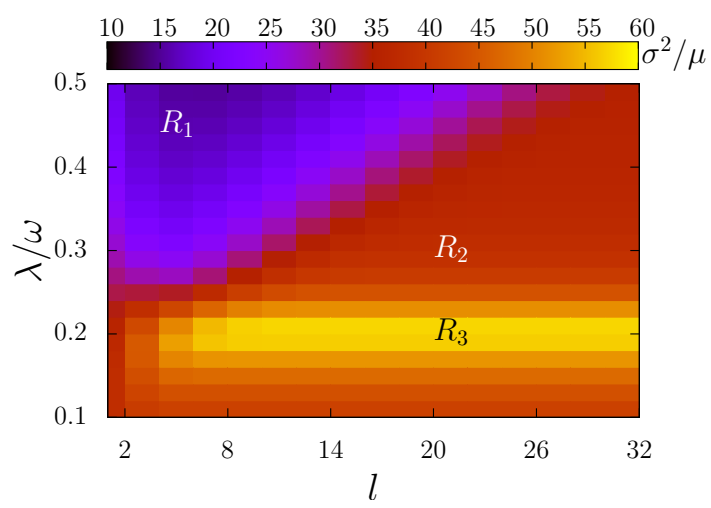

(b)

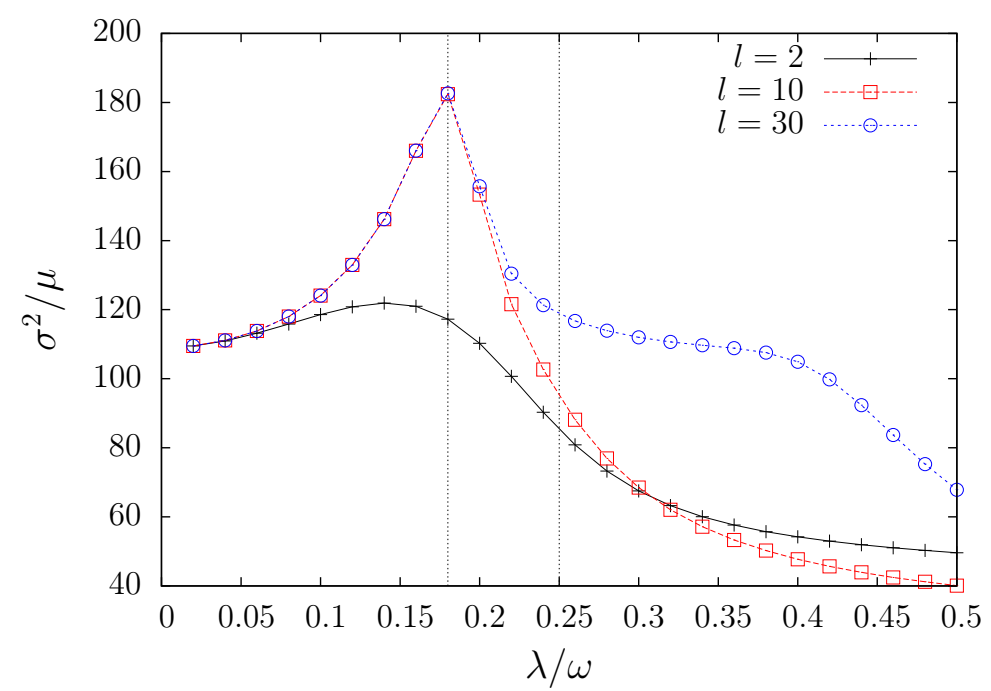

(c)

Figura 4.4: Fator de Fano $F_{N}(\lambda, l)$ para o estado fundamental. Em a) $N=10$ átomo enquanto em b) $N=8$. Na região $R_{1}\left(R_{3}\right)$, efeitos finitos são dominantes (desprezáveis). Na região intermediária $R_{2}$, incrementos em $\mu$ são seguidos por incrementos similares na variância $\sigma$, criando platôs. c) Fator de Fano para $N=10$ e valores de corte $l=2,10$ e 30. A linha tracejada indica o valor $\lambda_{c}$ onde $F_{N}$ desenvolve um máximo. Para $l \geqslant N$ e $\lambda<\lambda_{c}$, as propriedades do estado fundamental não são alteradas pelo corte $l$. 


\subsection{Simetria $U(1)$}

Apesar da definição do valor $\lambda_{c}$, por meio do estado fundamental, como estimador do impacto de efeitos finitos, não podemos inferir que o mesmo ocorre para o restante do autoespectro da Eq. (4.1). A fim de investigar o sistema como um todo, convém examinar as simetrias discretas da Eq. (4.1), em particular aquelas que se relacionam com os estados atômicos. Para isto, considere o operador de permutações cíclicas $\hat{P}$, que atua apenas sobre estados atômicos. Por exemplo, se o vetor $|\uparrow \downarrow \cdots \downarrow\rangle$ representa uma configuração atômica de $N$ átomos, na qual átomos em seu estado excitado são indicados pelo símbolo $\uparrow$. A ação de $\hat{P}$ sobre este vetor produz $\hat{P}|\uparrow \downarrow \cdots \downarrow\rangle=|\downarrow \uparrow \downarrow \cdots \downarrow\rangle$. Os autovetores do operador $\hat{P}$ são muito convenientes aqui pois quebram o operador $\hat{H}$ em $N$ blocos disjuntos. Estes blocos - ou setores, como também são conhecidos - são enumerados por um número quântico $p=0,1, \ldots, N-1$. Neste Capítulo, expressamos estes autovetores através de sua configuração explícita, seguida do número de permutação (ou momento) $p$ : $|\uparrow \downarrow \cdots \downarrow, p\rangle$, tal que $\hat{P}|\uparrow \downarrow \cdots \downarrow, p\rangle=\mathrm{e}^{-2 \imath \pi p / N}|\uparrow \downarrow \cdots \downarrow, p\rangle$. Veja o Apêndice 2 para uma discussão mais detalhada sobre essa construção.

Os operadores $\hat{J}^{ \pm, z}$ são simétricos quanto à ação da permutação cíclica, isto é, eles comutam com $\hat{P}$ : $\left[\hat{J}^{ \pm, z}, \hat{P}\right]=0$. A consequência desta conservação implica que o Hamiltoniano da Eq. (4.1) também pode ser escrito em setores de momento $p$. Em particular, o estado fundamental $\left|\psi_{0}\right\rangle$ encontra-se no setor $p=0$. A Figura 4.5 exibe o espectro de energia no setor $p=0$ e como a interação radiação-matéria quebra simetrias em relação ao problema não-interagente, conforme o acréscimo do corte no número de fótons $l$.

Efeitos finitos devido ao corte $l$ são verificados a partir da diferença de energia $\Delta E(\lambda)=E_{1}(\lambda)-E_{0}(\lambda)$, onde $E_{0}$ e $E_{1}(\lambda)$ são as energias do estado fundamental e do primeiro estado excitado, respectivamente, no setor $p=0$ como ilustra a Figura 4.6. De acordo com nossos resultados numéricos, o sistema desenvolve um gap de energia finito $\Delta E>0$ para $l>N$ e $\lambda \leqslant \lambda_{c}$. Para $\lambda>\lambda_{c}$, como indicado pelo gráfico da Figura 4.6b), o gap de energia diminui exponencialmente com o incremento do corte, sugerindo fortemente que tais correções desaparecem no limite termodinâmico.

No regime em que $\lambda$ se anula, a Eq. (4.1) conserva tanto o número médio de fótons quanto o número de átomos excitados. Essas regras de conservação podem 


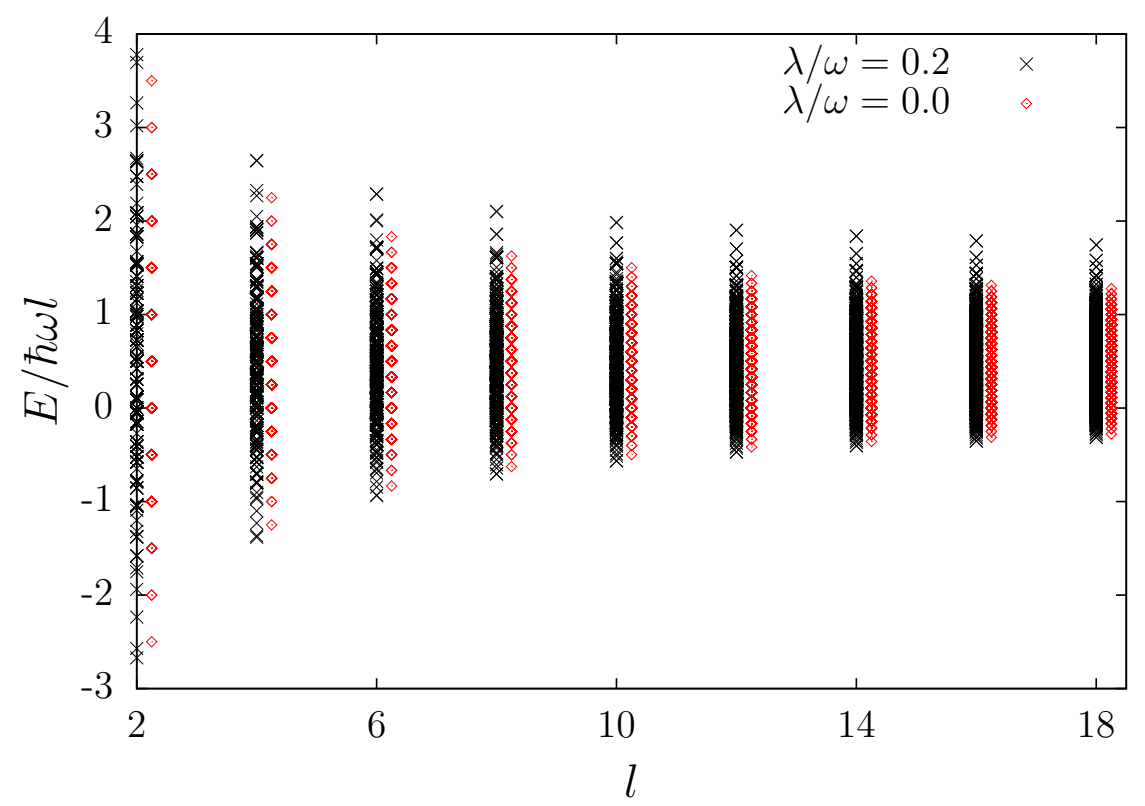

Figura 4.5: Degenerescência espectral. Espectro de energia normalizado para $N=10$ átomos $(\Omega=\omega)$ para diferentes valores de corte $l$, no setor de momento $p=0$. Os diamantes vermelhos são as energias no problema não-interagente, $\lambda / \omega=0$ e são ligeiramente deslocadas por $\delta l$ por questão de clareza. Neste regime, a menor (maior) energia vale $E_{\mp} / \hbar \omega l=\mp(N / 2 l)+(1 \mp 1) / 2$. As cruzes pretas mostram os níveis de energia para $\lambda / \omega=1 / 5$. A interação com a radiação levanta a degenerescência atômica e, concomitantemente, desloca os níveis de energia para baixo, conforme o acréscimo de $l$, produzindo densidades de fótons não-triviais.

ser traduzidas na simetria $U(1) \otimes U(1)$, que simplesmente expressa a conservação independente de partículas. Porém, para $\lambda$ não-nulo, esse cenário não se sustenta pois a razão $\langle n\rangle /\left\langle J^{z} / \hbar+N / 2\right\rangle$ depende do valor $\lambda / \omega$. A fim de clarificar essa afirmação, considere a ação da Eq. (4.1) no regime de acoplamento forte $\lambda \gg \omega=\Omega$ sobre o estado entrópico

$$
|\psi\rangle=\left[\prod_{\otimes}^{N} \frac{|\uparrow\rangle-|\downarrow\rangle}{\sqrt{2}}\right] \otimes\left[\frac{\sum_{m=0}^{l}|m\rangle}{\sqrt{l+1}}\right],
$$

que resulta na desigualdade

$$
-2 \hbar \lambda N l^{1 / 2} \leqslant\left\langle\psi\left|\lambda\left(\hat{a}^{\dagger}+\hat{a}\right)\left(\hat{J}^{+}+\hat{J}^{-}\right)\right| \psi\right\rangle \leqslant-\hbar \lambda N l^{1 / 2} .
$$

A manipulação algébrica da expressão acima produz $-2 \hbar \lambda N l^{1 / 2} \leqslant\langle\psi|\hat{H}| \psi\rangle-$ $\hbar \omega l / 2 \leqslant-\hbar \lambda N l^{1 / 2}$, cuja condição extrema ocorre em $N(\lambda / \omega)^{2} \leqslant 2\langle n\rangle / N \leqslant$ $4 N(\lambda / \omega)^{2}$.

A dependência aparente de $\left\langle J^{z}\right\rangle$ com $\langle n\rangle$ é clarificada a partir das transformações unitárias $\hat{U}(\theta)=\exp \left[\imath \theta\left(\hat{a}^{\dagger} \hat{a}-N / 2+\hat{J}^{z} / \hbar\right)\right]$. Sob a simetria $U(1)$, tanto matéria quanto campos de radiação modificam-se de maneira coerente, devido ao 


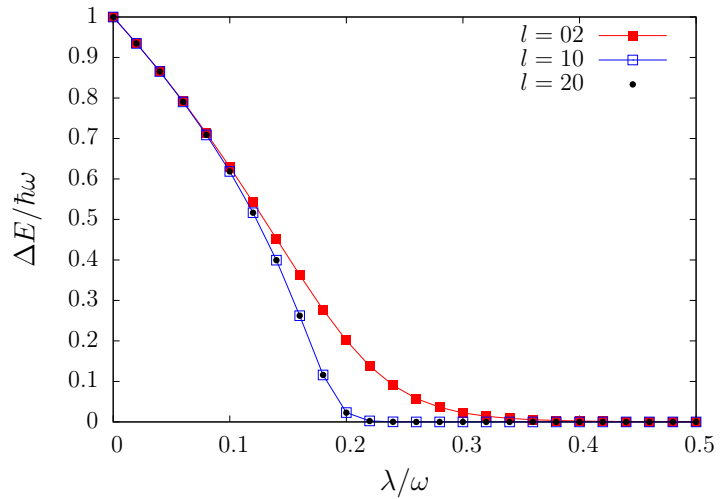

(a)

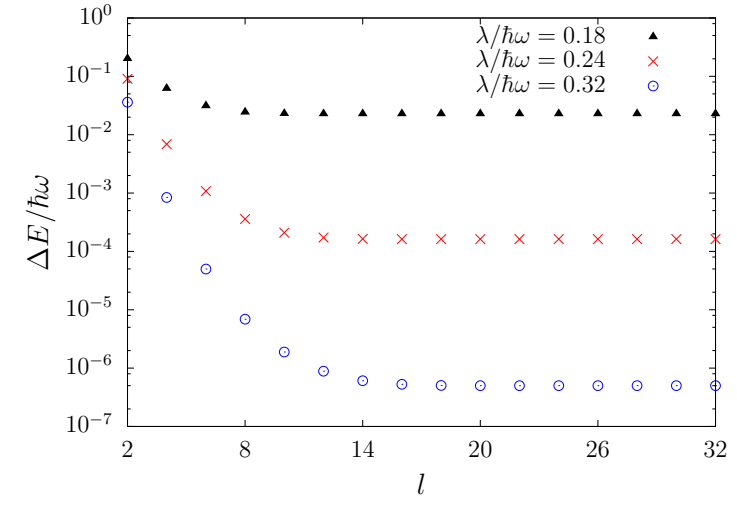

(b)

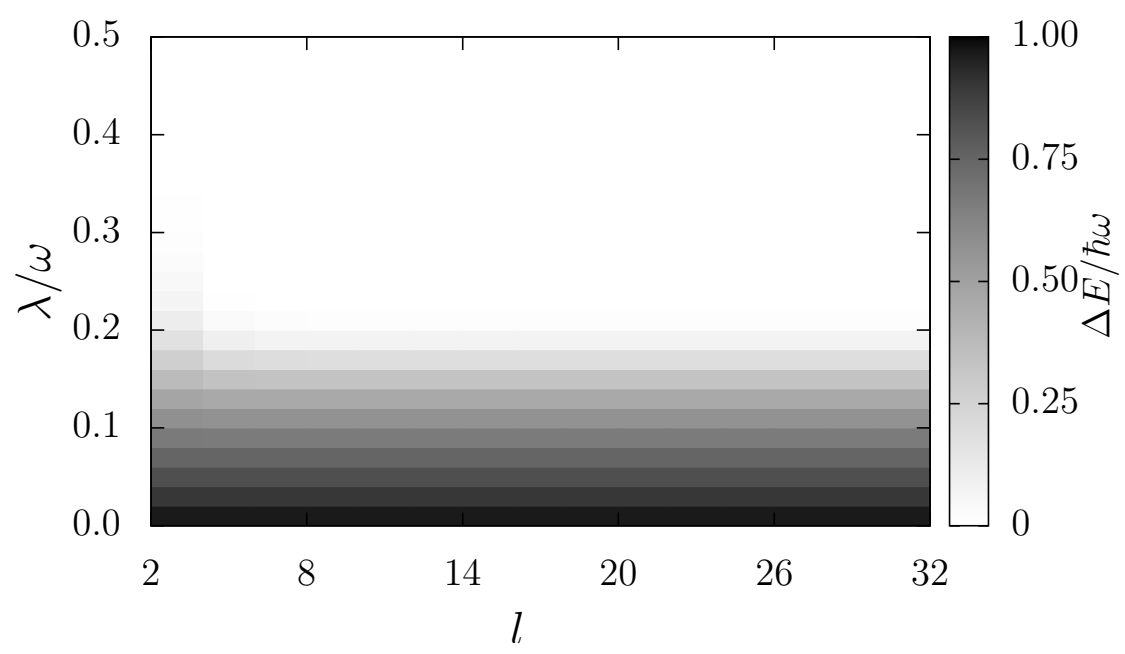

(c)

Figura 4.6: Gap de energia. Gap de energia para $N=10$ átomos $(\Omega=\omega)$ para diferentes valores de corte $l$, no setor de permutação $p=0$. a) Para $l \geqslant N$ e $\lambda<\lambda_{c}$, as curvas de diferença de energia colapsam, indicando a ausência de efeitos finitos devido ao número de fótons. O gap de energia eventualmente desaparece com o incremento de $\lambda$. b) Para $\lambda_{c}, \Delta E$ diminui exponencialmente com $\lambda$ e $l$. c) Mapa de cores para $\Delta E / \hbar \omega$ no setor $p=0$ sector. O valor $\lambda_{c}$ é o valor do acoplamento que separa a fase de $\Delta E$ finito daquela sem gap.

vínculo da cavidade, preservando a carga elétrica $q$ em seu interior. Por questão de simplicidade, considere o Hamiltoniano do modelo de Dicke não-interagente na Eq. (4.1). Ele claramente admite a simetria $U(1) \otimes U(1)$

$$
\hat{J}_{k}^{ \pm} \rightarrow \mathrm{e}^{\mp \imath \phi} \hat{J}_{k}^{ \pm}, \quad \hat{a} \rightarrow \mathrm{e}^{\imath \theta} \hat{a},
$$

onde as fases reais $\phi$ e $\theta$ identificam as transformações de gauge de primeiro tipo para os campos atômicos e de radiação, respectivamente. Estas transformações mantém as relações algébricas dos campos inalteradas. Ao mesmo tempo, a fundamentação de ambos operadores encontra-se na teoria eletromagnética e, portanto, as operações 
de simetria apenas expressam a conservação da carga elétrica. Nesse caso particular, como os campos não interagem, a carga é conservada de maneira independente para cada campo.

Para $\lambda \neq 0$, porém, ocorre a interação entre o momento de dipolo elétrico dos átomos e o campo de radiação. Na ausência de efeitos relativísticos e fótons de altas energias, eventos de criação de pares podem ser desprezados. Um resultado disso é que agora os campos de matéria e de radiação se amarram para garantir a conservação da carga elétrica total dentro da cavidade. Essa exigência introduz uma dependência mútua para as transformações de gauge, ou seja, $\phi \equiv \phi(\theta)$. Essa relação quebra a simetria $U(1) \otimes U(1)$ do caso não-interagente na simetria $U(1)$, gerando vínculos adicionais que no modelo de Dicke. Sob tais transformações, o

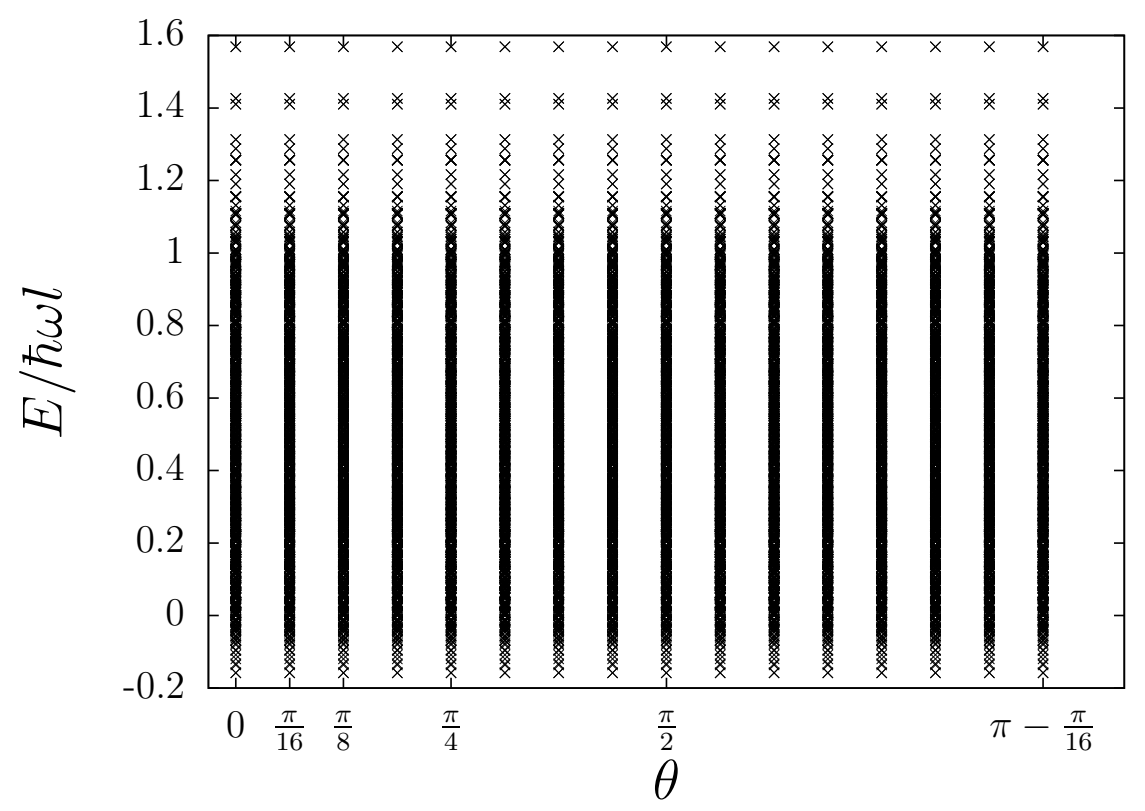

Figura 4.7: Invariância espectral por transformações de gauge. As autoenergias normalizadas em um sistema de $N=8$ átomos ( $\operatorname{corte} l=32, \lambda / \omega=1 / 4$ e setor de permutação $p=0$ ) se mantém inalteradas após as transformações de gauge de primeiro tipo, para para diversos valores do parâmetro $\theta$.

Hamiltoniano da Eq. (4.1) fica

$$
\begin{aligned}
\hat{H} & =\hat{H}_{0}+\lambda \sum_{k}^{N}\left(\hat{a} \hat{J}_{k}^{+} \mathrm{e}^{\imath(\theta-\phi(\theta))}+\mathrm{hc}\right)+\lambda \sum_{k}^{N}\left(\hat{a} \hat{J}_{k}^{-} \mathrm{e}^{\imath(\theta+\phi(\theta))}+\mathrm{hc}\right), \\
\hat{H}_{0} & =\hbar \omega \hat{a}^{\dagger} \hat{a}+\Omega \hat{J}^{z} .
\end{aligned}
$$

O espectro de energia no setor $p=0$ é exibido na Figura 4.7, normalizado pela máxima energia possível do campo de radiação, para diversos valores do parâmetro 
$\theta$. Conforme esperado, o espectro de energia é invariante por transformações de gauge e não dependem de nenhum valor particular de $\theta$ ou $\phi(\theta)$. Assim, podemos utilizar essa informação para determinar se existem vínculos adicionais e quais são suas expressões, especialmente em relação aos termos anti-girantes.

As duas observáveis mais convenientes desse problema são os números médios de fótons e a "magnetização" atômica, respectivamente, $\left\langle\hat{a}^{\dagger} \hat{a}\right\rangle$ e $\left\langle\hat{J}^{z}\right\rangle$. Essas quantidade são naturalmente invariantes por transformações de gauge de primeiro tipo, de maneira que $(d / d \theta)\left\langle\hat{a}^{\dagger} \hat{a}\right\rangle=(d / d \theta)\left\langle\hat{J}^{z}\right\rangle=0$. Convém, portanto, estudar suas equações de movimento a la Ehrenfest $[41], \imath \hbar(d / d t)\langle\hat{O}\rangle=\langle[\hat{O}, \hat{H}]\rangle$, em que $\hat{O}=\hat{a}^{\dagger} \hat{a}, \hat{J}^{z}$. Essas operações resultam em

$$
\begin{aligned}
-\frac{\hbar}{\lambda} \frac{d}{d \theta} \frac{d}{d t}\left\langle\hat{a}^{\dagger} \hat{a}\right\rangle=0 & =\sum_{k=1}^{N}\left\langle\left(1+\frac{d \phi}{d \theta}\right)\left(\hat{a} \hat{J}_{k}^{-} \mathrm{e}^{\imath(\theta+\phi)}+\hat{a}^{\dagger} \hat{J}_{k}^{+} \mathrm{e}^{-\imath(\theta+\phi)}\right)\right\rangle \\
& +\sum_{k=1}^{N}\left\langle\left(1-\frac{d \phi}{d \theta}\right)\left(\hat{a} \hat{J}_{k}^{+} \mathrm{e}^{\imath(\theta-\phi)}+\hat{a}^{\dagger} \hat{J}_{k}^{-} \mathrm{e}^{-\imath(\theta-\phi)}\right)\right\rangle . \\
-\frac{1}{\lambda} \frac{d}{d \theta} \frac{d}{d t}\left\langle\hat{J}^{z}\right\rangle=0 & =\sum_{k=1}^{N}\left\langle\left(1+\frac{d \phi}{d \theta}\right)\left(\hat{a} \hat{J}_{k}^{-} \mathrm{e}^{\imath(\theta+\phi)}+\hat{a}^{\dagger} \hat{J}_{k}^{+} \mathrm{e}^{-\imath(\theta+\phi)}\right)\right\rangle \\
& -\sum_{k=1}^{N}\left\langle\left(1-\frac{d \phi}{d \theta}\right)\left(\hat{a} \hat{J}_{k}^{+} \mathrm{e}^{\imath(\theta-\phi)}+\hat{a}^{\dagger} \hat{J}_{k}^{-} \mathrm{e}^{-\imath(\theta-\phi)}\right)\right\rangle .
\end{aligned}
$$

A partir das Eqs. (4.8a) e (4.8b), seleciona-se as escolhas dos gauges para os operadores de matéria e de radiação. De fato, descobre-se uma relação útil e relacionada com a RWA quando $d \phi / d \theta=1$ :

$$
\sum_{k=1}^{N}\left\langle\hat{a} \hat{J}_{k}^{-} \mathrm{e}^{2 \imath \theta}+\hat{a}^{\dagger} \hat{J}_{k}^{+} \mathrm{e}^{-2 \imath \theta}\right\rangle=0 .
$$

Fixando-se $\theta=m \pi, m=0, \pm 1, \ldots$, equivale a afirmar que a contribuição dos operadores anti-girantes se anula quando mediada. Uma outra forma de se expressar esse resultado consiste em assumir que não existe correlações entre os átomos e a radiação dentro da cavidade, consistente com as hipóteses da RWA. De fato, esse resultado enfatiza a grande aplicabilidade da RWA.

Outra propriedade é derivada a partir das equações de energia: o vínculo na Eq. (4.9) transforma a equação de autoenergia em um problema de minimização. Seja $\left|\varphi_{r}\right\rangle$ um autoestado do Hamiltoniano $\hat{H}$, sujeito à RWA, com autovalores $E_{r}^{\text {RWA }}$. Se a combinação linear $|\psi\rangle=\sum_{r} c_{r}\left|\varphi_{r}\right\rangle$, com a correspondente matriz densidade $\hat{\rho}$, satisfaz a Eq. (4.9), então a equação de energia se reduz a $\operatorname{Tr}\left[\rho\left(H-H^{\mathrm{RWA}}\right)\right]=0$, 
que é um funcional de energia para a solução $\hat{\rho},\langle E\rangle=\sum_{r} E_{r}^{\mathrm{RWA}} \rho_{r r}$.

\subsection{Operadores angulares}

Os efeitos atômicos mediados pelos campos de radiação são mais evidentes quando se considera os efeitos e vínculos impostos por rotações globais. Para este propósito, os operadores da Eq. (4.1) requerem transformações adicionas para exibir um conteúdo rotacional, preservando também o número quântico $p$. Nós começamos pelo caso no qual $0 \leq n \leq l$ fótons estão confinados na cavidade ótica sem átomos, $N=0$. Neste cenário, o momento angular total é $\Delta m=\hbar n$ e a energia vale $E=\hbar \omega n$. O espectro completo de energia é obtido variando-se $n=0,1, \ldots, l$; os autoestados correspondem à base do operador de momento angular orbital, $|l, m\rangle$, com $m=-l,-l+1, \ldots,+l$. Este modelo simples permite a identificação $q=l$ como o autovalor de momento angular total, enquanto $q_{z}=m$ corresponde à projetação no eixo z. Essa discussão é então generalizada para $N$ não-nulo. Para esse propósito, faz-se uso das transformações de Holstein-Primakoff [11], levando-se em conta a definição da carga conservada $q_{z}=\left\langle\hat{Q}^{z}\right\rangle / \hbar$, como já apresentado no Capítulo 2. Neste caso,

$$
\begin{gathered}
\hat{Q}^{z} \equiv \hat{L}^{z}+\hat{J}^{z}, \\
\hat{Q}^{+} \equiv \hat{L}^{+}+\hat{J}^{+}, \\
\hat{Q}^{-} \equiv \hat{L}^{-}+\hat{J}^{-},
\end{gathered}
$$

com $\hat{L}^{+}=\hbar \sqrt{l+1-\hat{a}^{\dagger} \hat{a}} \hat{a}^{\dagger}$ e $\hat{L}^{z}=\hbar\left(2 \hat{a}^{\dagger} \hat{a}-l\right)$. O operador $\hat{L}^{-}$é obtido através da conjugação Hermitiana de $\hat{L}^{+}$. Aqui, $l$ é valor do corte para o número de fótons aprisionados na cavidade para cada modo bosônico. A partir destas considerações, obtém-se $\left[\hat{Q}^{+}, \hat{Q}^{-}\right]=2 \hbar \hat{Q}^{z}$ e $\left[\hat{Q}^{z}, \hat{Q}^{ \pm}\right]= \pm \hbar \hat{Q}^{ \pm}$. Além disso, a identidade de Jacobi é satisfeita, $\left[\hat{Q}^{z},\left[\hat{Q}^{+}, \hat{Q}^{-}\right]\right]+\left[\hat{Q}^{-},\left[\hat{Q}^{z}, \hat{Q}^{+}\right]\right]+\left[\hat{Q}^{+},\left[\hat{Q}^{-}, \hat{Q}^{z}\right]\right]=0$, definindo, portanto, uma álgebra de Lie [15]. O maquinário algébrico construído até o momento encoraja a interpretação do operador $\hat{Q}^{z}$ como a projeção no eixo $z$ do operador de momento angular. Logo, os operadores radiativos são interpretados como operadores de momento angular orbital, enquanto os de matéria tem papel análogo ao spin. Essas afirmativas levam naturalmente à definição do operador de Casimir quadrado, $\hat{Q}^{2}=\left(\hat{Q}^{z}\right)^{2}+(1 / 2)\left\{\hat{Q}^{+}, \hat{Q}^{-}\right\}$. Vale a pena reforçar que utilizamos o corte finito para 
número de fótons para fundamentar as transformações de Holstein-Primakoff para o campo de fótons, enfatizando o papel do momento angular. ${ }^{3}$

As correções de efeito finito oriundas do corte $l$ aparecem nas Eqs. (4.10a)(4.10c) após o procedimento de linearização

$$
\hat{L}^{+}=\hbar \sqrt{l+1}\left(1-\frac{\hat{a}^{\dagger} \hat{a}}{2 l+2}\right) \hat{a}^{\dagger}+o\left(l^{-3 / 2}\right) .
$$

Em ordem $l^{-1}$, calcula-se o operador inverso $\left[1-\hat{a}^{\dagger} \hat{a} /(l+1)\right]^{-1 / 2}=1+\hat{a}^{\dagger} \hat{a} /(2 l+$ $2)+o\left(l^{-2}\right)$. Este operador permite reescrever os operadores bosônicos $\hat{a}^{\dagger}$ e $\hat{a}$ por meio de suas contrapartes orbitais $\hat{L}^{+}$e $\hat{L}^{-}$, respectivamente. Estes resultados provêm uma interpretação para os operadores girantes e anti-girantes: eles são operadores do tipo spin-órbita, o que favorece o emprego do operador de Casimir quadrático e leva a duas importantes conclusões. Primeiro, os operadores girantes são diagonais

$$
\hat{a} \hat{J}^{+}+\hat{a}^{\dagger} \hat{J}^{-} \approx \frac{1}{\hbar \sqrt{l+1}}\left[\left(\hat{Q}^{2}-\left(\hat{Q}^{z}\right)^{2}-2 \hat{L}^{z} \hat{J}^{z}\right)\left(1+\frac{l}{4(l+1)}+\frac{\hat{L}^{z}}{2 \hbar(l+1)}\right)\right] .
$$

Esta expressão reflete a simplicidade dos operadores girantes e de suas contribuições, em concordância com as asserções de que a RWA é incapaz de intermediar interações complexas (e interferência) no gás atômico. Segundo, os operadores anti-girantes produzem contribuições não-diagonais

$$
\hat{a}^{\dagger} \hat{J}^{\dagger}+\hat{a} \hat{J} \approx \frac{2}{\hbar \sqrt{l+1}}\left[\left(\hat{L}^{x} \hat{J}^{x}-\hat{L}^{y} \hat{J}^{y}\right)\left(1+\frac{l}{4(l+1)}+\frac{\hat{L}^{z}}{2 \hbar(l+1)}\right)\right],
$$

os quais, ao contrário de (4.12), acoplam diferentes setores de $q_{z}$ para dado $q$.

\subsection{Duas espécies atômicas}

As ideias ao redor dos operadores de momento angular como elementos centrais em generalizações modelo de Dicke podem ser aplicadas a sistemas com duas espécies atômicas de dois níveis, $\xi=1,2$. Conforme esquematizado pela Figura 4.8,

\footnotetext{
${ }^{3}$ No limite termodinâmico, o costume é utilizar o número total de átomos e transformar o problema em osciladores harmônicos acoplados $[146,147]$. Ao fazer essa escolha, o comportamento bosônico da parte atômica é favorecido em detrimento da contraparte fermiônica. Essa opção é compatível com intensa agitação térmica, onde as excitações coletivas melhor caracterizam o sistema de $N$ átomos. Não se deve confundir esse caso com condensação de BoseEinstein, que introduz elevado grau de coerência entre os entes componentes. Baseado nesses argumentos, temos a opinião que esse cenário não é consistente com os experimentos e estudos mais recentes de superradiância [27, $28,142,155,156]$, posto que enfatiza modos de altas frequências e, portanto, comprimentos de onda muito curtos, o que intuitivamente resulta em baixa coerência entre os estados atômicos. Mais recentemente, a possibilidade de superradiância por modos fermiônicos foi investigada na Ref. [157]. Em nossa abordagem, evitamos essa escolha pois transformamos o campo bosônico radiativo em um outro modo igualmente bosônico.
} 
a cavidade óptica não dissipativa confina duas espécies atômicas ultrafrias, as quais interagem apenas com os modos radiativos correspondentes, cujas energias são $\hbar \omega_{\xi}$ e spin $m_{\xi}=(-1)^{\xi-1}$. Claro, trata-se de uma idealização bastante grosseira vez que campos de radiação perturbam os autoestados naturais dos sistemas atômicos. A ideia aqui é investigar se as leis de conservação do momento angular do sistema produzem interferência entre as duas espécies, que de outra forma seriam não-interagentes. Em resumo, a cavidade confina duas espécies de átomos e dois modos bosônicos. Assim como no caso anterior, a cavidade impõe vínculos sobre a matéria e radiação em seu interior.

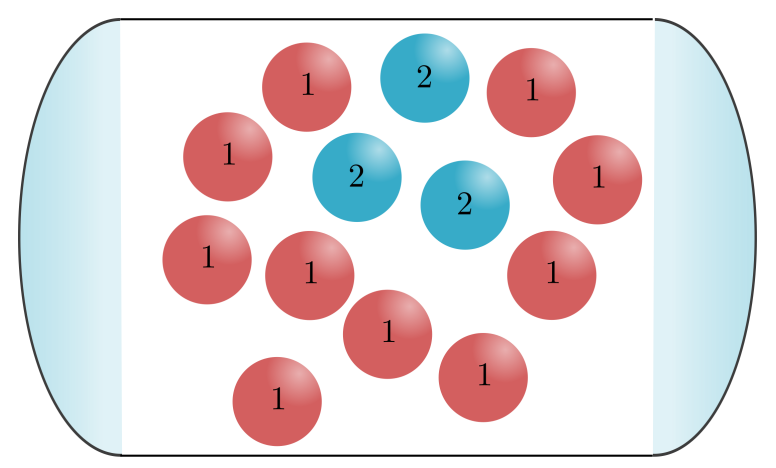

Figura 4.8: Duas espécies de atômicas confinadas numa cavidade óptica idealizada não-dissipativa. Cada tipo de átomo é identificado pelo inteiro $\xi=1,2$ e interage apenas com a radiação eletromagnética ressonante correspondente. Átomos do tipo $\xi=1(\xi=2)$ absorvem apenas fótons com energia $\hbar \omega_{1}\left(\hbar \omega_{2}\right)$ e spin $m=+1(m=-1)$.

Seguindo os argumentos da seção anterior, podemos definir as transformações de gauge para cada espécie atômica. A diferença se dá quando ambas as nuvens atômicas estão presentes, pois a superposição dos campos eletromagnéticos dentro da cavida também acopla os parâmetros das transformações unitárias $\theta_{1,2}$. Esta é a condição necessária para garantir a conservação da carga elétrica da teoria. Assim, podemos concluir que $\theta_{1}=\theta_{2}=\theta$. Esta propriedade dita que as densidades para as diferentes espécies atômicas não são completamente descorrelacionadas: a Eq. (4.9) correspondente a cada espécie estão acopladas pelo parâmetro compartilhado $\theta$. Por exemplo, dada uma matriz densidade $\rho_{1}^{\prime}$ correspondente à espécie $\xi=1$, encontra-se um valor adequado $\theta^{\prime}$ como solução da Eq. (4.9). A fim de obter a solução completa, resolve-se a Eq. (4.9), agora com $\theta^{\prime}$ fixo, de onde se determina a matriz densidade $\rho_{2}^{\prime}$ correspondente à espécie $\xi=2$. Assim, as soluções obtidas para uma espécie de certa maneira restringem o espaço de soluções da outra espécie atômica. 
Assim como no caso anterior, iniciamos nossa análise considerando o caso onde $0 \leq n_{\xi} \leq l$ fótons estão confinados dentro de uma cavidade óptica sem átomos $N_{1}=N_{2}=0$, produzindo momento angular líquido $\Delta m=\hbar\left(n_{1}-n_{2}\right)$ e energia $E=\hbar \omega_{1} n_{1}+\hbar \omega_{2} n_{2}$. Novamente, identifica-se $q=l$ e $q_{z}=\Delta m$ como os autovalores de momento angular total e projetado, respectivamente. Estes resultados são generalizados para valores não-nulos de $N_{\xi}$ por meio das Eqs. (4.8a) e (4.8b), para ambos tipos de espécie atômica. A escolha mais intuitiva aqui resume-se a tomar $q_{z}=\sum_{\xi}\left\langle\hat{a}_{\xi}^{\dagger} \hat{a}_{\xi}+\hat{J}_{\xi}^{z} / \hbar\right\rangle$. Porém, essa escolha só é viável em conjunção com a RWA, em concordância com a Eq. (4.9). As transformações Holstein-Primakoff são utilizadas novamente com carga $q_{z}=\left\langle\hat{Q}^{z}\right\rangle / \hbar$, tal que

$$
\begin{aligned}
& \hat{Q}^{z} \equiv \hbar\left(\hat{a}_{1}^{\dagger} \hat{a}_{1}-\hat{a}_{2}^{\dagger} \hat{a}_{2}\right)+\hat{J}_{1}^{z}-\hat{J}_{2}^{z}, \\
& \hat{Q}^{+} \equiv \hat{L}_{1, l}^{+}+\hat{L}_{2, l}^{-}+\hat{J}_{1}^{+}+\hat{J}_{2}^{-}, \\
& \hat{Q}^{-} \equiv \hat{L}_{1, l}^{-}+\hat{L}_{2, l}^{+}+\hat{J}_{1}^{-}+\hat{J}_{2}^{+} .
\end{aligned}
$$

onde $\hat{L}_{1, l}^{+}=\hbar \sqrt{l+1-\hat{a}_{1}^{\dagger} \hat{a}_{1}} \hat{a}_{1}^{\dagger}$ e $\hat{L}_{2, l}^{-}=\hbar \hat{a}_{2} \sqrt{l+1-\hat{a}_{2}^{\dagger} \hat{a}_{2}}$. As Eqs. (4.14a)-(4.14c) geram as estruturas algébricas idênticas àquelas do case de uma única espécie, resultando em igual interpretação para $\hat{Q}^{z}$ e para o operador de Casimir quadrático $\hat{Q}^{2}=\left(\hat{Q}^{z}\right)^{2}+(1 / 2)\left\{\hat{Q}^{+}, \hat{Q}^{-}\right\}$.

O novo espaço de Hilbert e os operadores angulares correspondentes, $\hat{Q}$, são candidatos naturais para estudar a interferência entre as nuvens atômicas, especialmente em situações de forte correlação como na fase superradiante. Esta fase é caracterizada por um grande armazenamento de energia nos campos de matéria (átomos excitados), que então é descarregado de maneira coerente como um pulso de curta duração. Para a cavidade ideal, isso implica que a energia disponível a partir de fontes eletromagnéticas é menor que aquelas em fases não-superradiantes. Assim, existe previsão que o número médio de fótons $n_{\xi}$ seja muito menor que o corte $l$ para fótons dentro da cavidade, ou seja, $\delta n_{\xi}=\left\langle\hat{a}_{\xi}^{\dagger} \hat{a}_{\xi}\right\rangle / l \ll 1$. Esta observação justifica o procedimento de linearização das transformações de Holstein-Primakoff para valores arbitrários de $N_{\xi}$, já que $l$ é o único parâmetro relevante, compartilhado por ambos. Desta forma, temos

$$
\hat{L}_{1, l}^{+} \approx \hbar \sqrt{l+1}\left(1-\frac{\hat{a}_{1}^{\dagger} \hat{a}_{1}}{2 l+2}\right) \hat{a}_{1}^{\dagger} .
$$

Expansões análogas são obtidas para o restante dos operadores levantamento e abai- 
xamento $\hat{L}_{\xi, l}^{ \pm}$.

Novamente, até ordem $l^{-1}$, a inversão do operador $\left[1-a_{\xi}^{\dagger} a_{\xi} /(l+1)\right]^{-1 / 2}$ pro$\operatorname{duz} 1+a_{\xi}^{\dagger} a_{\xi} /(2 l+2)+o\left(l^{-2}\right)$, permitindo a substituição dos operadores bosônicos $\hat{a}_{\xi}^{\dagger}$ e $\hat{a}_{\xi}$ por suas respectivas contrapartes orbitais $\hat{L}_{\xi, l}^{+}$e $\hat{L}_{\xi, l}^{-}$, respectivamente. Assim como no caso anterior, as contribuições girantes e anti-girantes são interpretadas como operadores spin-órbita. Todavia, diferentemente do caso anterior, a ação destes operadores conectam a evolução temporal das duas espécies atômicas via campo eletromagnético compartilhado, muito embora os átomos propriamente ditos não tenham interação direta.

Devido à conservação de $q_{z}$ e $q$, as contribuições anti-girantes agora exigem o emprego do operador $\hat{Q}^{2}$ e sua base de autovetores correspondente. Esta exigência mistura ambos estados atômicos, o que em troca permite a interferência interatômica. Como exemplo, considere o caso onde o sistema encontra-se inicialmente na configuração que contém uma espécie atômica na fase superradiante enquanto a outra encontra-se completamente descarregada, ambas com $N$ átomos. Por questão de simplicidade, vamos assumir que $N$ fótons com spin $m=-1$ estão disponíveis dentro da cavidade. Essa configuração é representada pelo vetor $|N, 0 ; 0, N\rangle \operatorname{com} q_{z}=0$. Após um intervalo de tempo $t$, há possibilidade de se medir o estado $|0, N ; N, 0\rangle$. O estado $|0,0 ; 0,0\rangle$ também carrega $q_{z}=0$ e pode também poderia ter sido medido. Enquanto o primeiro evento é esperado no contexto de RWA, incluindo aí a interpretação simples de absorção-emissão, a segunda possibilidade é um desenlace comum na teoria do momento angular. A energia é conservada pois o operador de Casimir e os operadores spin-órbita comutam entre si. De fato, a Eq. (4.9) nos informa que os operadores anti-girantes produzem contribuições nulas para a energia, pois mistura diversos estados com o mesmo número quântico $q$.

\subsection{Conclusão}

Neste estudo idealizado, mostramos o papel desempenhado pelas leis de conservação no modelo de Dicke. A simetria $U(1)$, apesar de sua simplicidade, impõe uma forte restrição para os operadores anti-girantes, correlacionando as componentes de matéria, de tal forma que suas contribuições para a energia anulam-se, em média. Quando examinada sob o ponto de vista da conservação do momento angular 
total, mostramos que os termos de interação radiação-matéria equivalem a operadores spin-órbita. Tal fato permite a identificação dos princípios que guiam a interação indireta entre duas espécies atômicas não-interagentes umas com as outras. Devemos enfatizar que a exigência de cavidade não-dissipativas não é factível na prática: cavidades ópticas sempre dissipam energia, incluindo ai, absorção da radiação pelo material constituinte da cavidade, transmissão de luz, radiação de corpo negro, etc. A fim de levar em conta efeitos dissipativos, tanto em relação à energia quanto à coerência, deve-se adicionar os operadores de Lindblad, levando a uma versão dissipativa da Eq. (4.9). Em adição, tal generalização também estende o arcabouço programático do sistema ao adicionar uma propriedade que lembra torques para a equação de movimento, criando transições entre estados com diferentes valores de $q$. 


\section{5}

\section{Nanoestruturas magnéticas}

Se eu conseguisse explicá-la para uma pessoa mediana, ela não seria digna de um prêmio Nobel.

- Richard Feynman

Nanomateriais são aglomerados de átomos ou moléculas que formam estruturas cristalinas e apresentam propriedades físicas intermediárias entre aquelas do estado atômico e do regime macroscópico [158, 159]. São caracterizados pela limitação espacial de suas dimensões geométricas, as quais se estendem entre 1-100nm, em uma ou mais direções. Em virtude da finitude do sistema, efeitos de localização e confinamento espacial tornam-se extremamente relevantes [160]. A densidade de estados eletrônicos sofre alterações, tornando-se mais acentuada ao redor de determinados valores de energia, evidenciando a discretização dos níveis de energia. Isso permite a modelagem das interações microscópicas do sistema através de potenciais confinantes efetivos, tal qual o poço de potencial. As dimensões espaciais do material, portanto, desempenham um papel não trivial e tornam-se um parâmetro relevante para a caracterização de suas propriedades físico-químicas [161].

Neste estudo, investigamos as propriedades físicas de um sistema composto por duas nanoestruturas magnéticas, separadas espacialmente por um meio material dielétrico. Individualmente, cada nanoestrutura apresenta um rico espectro de energia e conteúdo termodinâmico. A introdução de um dielétrico, confinado entre ambas, permite a mediação de excitações pelas partes componentes do sistema. A fim de compreender as mudanças emergentes, torna-se necessário investigar os mecanismos microscópicos das interações presentes e o papel da finitude do sistema 
como elemento controlador de propriedades físicas. Em particular, estamos interessados nas características de caráter organizacional, como ordenamento magnético, e de transporte, como correntes de tunelamento entre as nanoestruturas.

A fim de compreender a dinâmica microscópica, as nanoestruturas magnéticas serão modeladas como uma composição de átomos dispostos em uma cadeia linear com condições periódicas de contorno [162, 163]. Estas estruturas recebem o nome de cadeias de spin quando os graus de liberdade relevantes podem ser efetivamente descritos por estados discretos, como descrevemos a seguir. Considere um conjunto de átomos com propriedades magnéticas, interagentes e alinhados numa dada direção espacial. Cada átomo encontra-se localizado em torno de uma região, ou sítio, que demarca o ponto de equilíbrio ou mínimo potencial coulombiano entre vizinhos. Os sítios possuem separação interatômica $a$ e são identificados pelo índice $k=1,2, \ldots, L$. Este sistema forma uma cadeia ou rede unidimensional cuja energia potencial possui periodicidade $a$, e que, em princípio, contempla as interações coulombianas inter e intra atômicas (elétron-elétron, núcleo-elétron, núcleo-núcleo).

O tratamento exato do problema de muitos corpos é extremamente complexo e, portanto, aproximações são introduzidas para reproduzir a dinâmica apenas dos elétrons mais fracamente ligados, responsáveis pelas propriedades de condução térmica e elétrica, etc. Por exemplo, elétrons em metais são modelados como um gás de partículas livres, enquanto semicondutores apresentam uma barreira de potencial para condução elétrica. Nestas aproximações de partículas quase livres, o spin eletrônico não desempenha papel relevante na formação de domínios magnéticos. De fato, a magnetização emerge devido às correlações de longo alcance entre elétrons da rede, oriundas do potencial coulombiano. O hamiltoniano não-relativístico para um sistema de elétrons é dado por

$$
\hat{H}=\sum_{i}\left[\frac{\hat{p}_{i}^{2}}{2 m}+\hat{V}\left(\hat{\vec{r}}_{i}\right)\right]+\frac{1}{2} \sum_{i \neq j} \frac{e^{2}}{\left|\hat{\vec{r}}_{i}-\hat{\vec{r}}_{j}\right|} .
$$

O índice $i$ refere-se ao $i$-ésimo elétron, $-e$ é a carga elementar do elétron, $\hat{\vec{r}}_{i}$ é o operador posição do $i$-ésimo elétron e $\hat{V}\left(\hat{\vec{r}}_{i}\right)$ é a energia potencial elétron-núcleo. A segunda parcela do lado direito da expressão acima é a energia de interação entre os elétrons. A interação entre elétrons de sítios vizinhos, da ordem de meV, é aquela que transmite vibrações pela rede e também responsável por fenômenos coletivos 
como a magnetização.

O hamiltoniano da Eq. (5.1) possui uma parcela, $\hat{H}_{0}$, que descreve a dinâmica de elétrons não interagentes sob ação de potenciais periódicos em uma rede, e outra, $\hat{H}_{1}$, que descreve o comportamento de elétrons interagentes, tal que $\hat{H}=\hat{H}_{0}+\hat{H}_{1}$. O autovetor $|m\rangle$ associado ao operador $\hat{H}_{0}$ descreve o estado de partículas livres e dá origem à banda $m$ de energia $\varepsilon_{m}$ observada em metais e semicondutores. Pelo formalismo da segunda quantização, o hamiltoniano (5.1) é descrito pelos operadores fermiônicos de criação e destruição de partículas livres, $\hat{a}_{m}^{\dagger}$ e $\hat{a}_{m}$, respectivamente,

$$
\hat{H}=\sum_{m, n}\left\langle m\left|\hat{H}_{0}\right| n\right\rangle \hat{a}_{m}^{\dagger} \hat{a}_{n}+\frac{1}{2} \sum_{m_{1}, m_{2}} \sum_{n_{1}, n_{2}}\left\langle m_{1} m_{2}\left|\hat{H}_{1}\right| n_{1} n_{2}\right\rangle \hat{a}_{m_{1}}^{\dagger} \hat{a}_{m_{2}}^{\dagger} \hat{a}_{n_{1}} \hat{a}_{n_{2}}
$$

Os operadores de criação (destruição) criam (destroem) a função de onda $\psi_{m}(\vec{r})$ da $m$-ésima banda de energia tal que

$$
\left\langle m\left|\hat{H}_{0}\right| n\right\rangle=\int d \vec{r} d \vec{r}^{\prime} \psi_{m}^{*}(\vec{r})\left\langle\vec{r}\left|\hat{H}_{0}\right| \vec{r}^{\prime}\right\rangle \psi_{n}\left(\vec{r}^{\prime}\right)=\varepsilon_{m} \delta_{m n} .
$$

A interação entre as diversas bandas impossibilita qualquer tentativa em extrair o comportamento essencial dos elétrons. Para baixas temperaturas, as características de sistemas monovalentes são majoritariamente influenciadas por uma única banda. Despreza-se, então, as contribuições de todas as bandas, exceto a dominante. Essa é a aproximação de banda única. Na representação de Bloch ou Fourier da Eq. (5.2) e considerando-se explicitamente os spins eletrônicos $s= \pm 1 / 2$, temos

$$
\hat{H}=\sum_{\vec{k}, s} \varepsilon_{\vec{k}} \hat{a}_{\vec{k} s}^{\dagger} \hat{a}_{\vec{k} s}+\sum_{\{k, s\}} \Gamma_{14}^{23} \hat{a}_{\overrightarrow{k_{1} s_{1}}}^{\dagger} \hat{a}_{\overrightarrow{k_{2} s_{2}}}^{\dagger} \hat{a}_{\overrightarrow{k_{3} s_{3}}} \hat{a}_{\overrightarrow{k_{4} s_{4}}}
$$

onde o segundo somatório é realizado sobre todos os possíveis valores de vetor de onda da rede cristalina $\vec{k}_{i}$ e spin $s_{i}$. As energias $\varepsilon_{\vec{k}}$ determinam as banda de energia. Os operadores $\hat{a}_{\vec{k} s}^{\dagger}$ criam elétrons com vetor de onda $\vec{k}$ e spin $s$. O acoplamento $\Gamma_{14}^{23}$ contém as contribuições da interação elétron-elétron. Retornando ao espaço real com as funções de Wannier [164], o segundo termo do lado direito da expressão acima é dado por

$$
\frac{1}{2} \sum_{i, j, k, l} \sum_{s, s^{\prime}}\left\langle i j\left|\frac{e^{2}}{r}\right| k l\right\rangle \hat{a}_{i s}^{\dagger} \hat{a}_{j s^{\prime}}^{\dagger} \hat{a}_{k s^{\prime}} \hat{a}_{l s},
$$

onde a soma $i, j, k, l$ é realizada sobre todos os sítios e $\hat{a}_{j s^{\prime}}$ são operadores que criam elétrons na banda de valência localizados no sítio $j$ com spin $s$. Através da relação de anticomutação, $\left\{\hat{a}_{j s}^{\dagger}, \hat{a}_{j s^{\prime}}\right\}=\delta_{j j^{\prime}} \delta_{s s^{\prime}}$, é possível identificar dois tipos de contribuição. 
A primeira é $n_{i s} n_{i s^{\prime}} \delta_{s,-s^{\prime}}$ e envolve spins antiparalelos no mesmo sítio, enquanto a segunda é

$$
\hat{H}_{\text {spin }}=-\sum_{i, j} \sum_{s, s^{\prime}} J_{i j} \hat{a}_{i s}^{\dagger} \hat{a}_{i s^{\prime}} \hat{a}_{j s^{\prime}}^{\dagger} \hat{a}_{j s}
$$

onde $J_{i j}$ é a energia de troca entre sítio $i$ e $j$.

Exceto pelo operador $\hat{H}_{\text {spin }}$, descrito pela Eq. (5.6), todos as outras contribuições são independentes do spin. Isto significa que $\hat{H}_{\text {spin }}$ contém em si o princípio de exclusão de Pauli para sítios suficientemente próximos, e é o único termo capaz de alterar a magnetização do meio. Por fim, identifica-se os operadores $\hat{a}_{i s}^{\dagger} \hat{a}_{i s^{\prime}}$ com as matrizes de Pauli localizadas no sítio $i, \hat{\boldsymbol{\sigma}}_{i}=\left(\hat{\sigma}_{i}^{x}, \hat{\sigma}_{i}^{y}, \hat{\sigma}_{i}^{z}\right)$, tal que

$$
\hat{H}_{\text {spin }}=-\frac{1}{4} \sum_{i, j \neq i} J_{i j} \hat{\boldsymbol{\sigma}}_{i} \cdot \hat{\boldsymbol{\sigma}}_{j}
$$

Na base $\left|\uparrow_{j}\right\rangle$ e $\left|\downarrow_{j}\right\rangle$, as matrizes de Pauli localizadas são

$$
\hat{\sigma}_{j}^{x}=\left(\begin{array}{cc}
0 & 1 \\
1 & 0
\end{array}\right), \hat{\sigma}_{j}^{y}=\left(\begin{array}{cc}
0 & -i \\
i & 0
\end{array}\right), \hat{\sigma}_{j}^{z}=\left(\begin{array}{cc}
1 & 0 \\
0 & -1
\end{array}\right),
$$

que são os geradores do grupo $S U(2)$, possuem autovalores $m= \pm 1$, e satisfazem a seguinte regra de comutação $\left[\hat{\sigma}_{j}^{\mu}, \hat{\sigma}_{i}^{\nu}\right]=2 i \sum_{\gamma} \varepsilon_{\mu \nu \gamma} \hat{\sigma}_{j}^{\gamma} \delta_{j i}$, com $\mu, \nu, \gamma=x, y, z$ e $\varepsilon_{\mu \nu \gamma}$ é o tensor anti-simétrico. Adicionalmente, as matrizes de Pauli ainda podem ser escritas como combinação dos operadores de levantamento $\hat{\sigma}_{j}^{+}$e abaixamento $\hat{\sigma}_{j}^{-}$de spins localizados: $\hat{\sigma}_{j}^{x}=\hat{\sigma}_{j}^{+}+\hat{\sigma}_{j}^{-}, \hat{\sigma}_{j}^{y}=-i\left(\hat{\sigma}_{j}^{+}-\hat{\sigma}_{j}^{-}\right), \hat{\sigma}_{j}^{z}=2 \hat{\sigma}_{j}^{+} \hat{\sigma}_{j}^{-}-1$ e que satisfazem a regra de anticomutação local, $\left\{\hat{\sigma}_{j}^{+}, \hat{\sigma}_{j}^{-}\right\}=1$.

O operador $\hat{H}_{\text {spin }}$ é geralmente escrito numa forma que o associa diretamente ao spin eletrônico. Nesta forma, recebe o nome de hamiltoniano de Heisenberg [30]

$$
\hat{H}=-\sum_{i, j \neq i} J_{i j} \hat{\boldsymbol{S}}_{i} \cdot \hat{\boldsymbol{S}}_{j}
$$

onde $\hat{\boldsymbol{S}}_{j}=(\hbar / 2) \hat{\boldsymbol{\sigma}}_{j}$ é o operador de spin do sítio $\vec{r}_{j}$ e $J_{i j}=J\left(\left|\vec{r}_{i}-\vec{r}_{j}\right|\right)$ fornece a intensidade média do acoplamento coulombiano entre dois sítios, e cujo valor é da ordem 4-7 meV. Em uma dimensão, o hamiltoniano de Heisenberg descreve uma cadeia de spin [17]. Os graus de liberdade eletrônico foram sistematicamente reduzidos, simplificados e mediados a fim de se obter uma descrição para fenômenos coletivos, em uma escala de energia da ordem de meV. 


\subsection{Cadeias de spin}

Os estados físicos associados às cadeias de spin contém tipicamente competição do tipo ordem-desordem. A ordem é caracterizada pelo alinhamento paralelo de spins e formação de domínios magnéticos. Suas excitações elementares são as ondas de spin, possuem comprimento típico maior que o espaçamento interatômico e baixa energia de ativação [165]. São capazes de alterar a magnetização local de spins presentes numa dada vizinhança, aumentando a desordem do sistema o que determina a formação e permanência de domínios magnéticos. Neste contexto, é intuitivo esperar que duas cadeias de spin com magnetizações distintas, ou diferentes níveis de ordem, entrem em equilíbrio caso possam interagir com um meio em comum, que passa a desempenhar um papel de mediador de excitações, acoplando as cadeias [166]. Essa transmissão pode ser interpretada como tunelamento de spins entre cadeias ou como uma corrente de spins [167-169].

A evolução temporal de uma cadeia de spin, de comprimento $L$ e com espaçamento interatômico $a$, é inteiramente descrita pelo hamiltoniano $\hat{H}_{\mathrm{sc}}$. Este por sua vez é composto pelo produto dos operadores de spin localizados $\hat{\boldsymbol{\sigma}}_{k}=$ $\left(\hat{\sigma}_{k}^{x}, \hat{\sigma}_{k}^{y}, \sqrt{\Delta} \hat{\sigma}_{k}^{z}\right)$, que são as matrizes de Pauli localizadas no sítio $k(k=1,2, \ldots, L)$, conforme regra a Eq. (5.8), com o vínculo de periodicidade $\hat{\boldsymbol{\sigma}}_{L+1}=\hat{\boldsymbol{\sigma}}_{1}$. O parâmetro $\Delta \in \mathbb{R}$ descreve a presença de um eixo preferencial de alinhamento para os spins. A intensidade do campo magnético externo na região do sítio $k$ é descrita por $\boldsymbol{h}_{k}=\left(h_{k}^{x}, h_{k}^{y}, h_{k}^{z}\right)$ e $\mu_{B}$ é o magneton de Bohr. Desta maneira, seguindo os moldes da Eq. (5.9),

$$
\hat{H}_{\mathrm{sc}}=-J \frac{\hbar^{2}}{4} \sum_{k=1}^{L}\left(\hat{\boldsymbol{\sigma}}_{k+1} \cdot \hat{\boldsymbol{\sigma}}_{k}\right)-\frac{\mu_{B} \hbar}{2} \sum_{k=1}^{L}\left(\boldsymbol{h}_{k} \cdot \hat{\boldsymbol{\sigma}}_{k}\right) .
$$

Os produtos escalares dos operadores de spin, $\hat{\boldsymbol{\sigma}}_{k+1} \cdot \hat{\boldsymbol{\sigma}}_{k}$, podem ser convenientemente decompostos em componentes difusivas e potenciais: $\left(\hat{\boldsymbol{\sigma}}_{k+1} \cdot \hat{\boldsymbol{\sigma}}_{k}\right) / 2=$ $\hat{\sigma}_{k+1}^{+} \hat{\sigma}_{k}^{-}+\hat{\sigma}_{k}^{+} \hat{\sigma}_{k+1}^{-}+2 \Delta \hat{n}_{k+1} \hat{n}_{k}-\Delta \hat{n}_{k+1}-\Delta \hat{n}_{k}+\Delta$. O operador localizado de número de partículas é obtido por meio da seguinte composição $\hat{n}_{k}=\hat{\sigma}_{k}^{+} \hat{\sigma}_{k}^{-}$. Seus autovalores são 0 e 1 para sítios com spin $m_{z}=-1 / 2,+1 / 2$, respectivamente. Há, portanto, uma interpretação conveniente para os operadores de levantamento (abaixamento) como operadores de criação (destruição) de partículas sem spin onde a ocupação nula (unitária) é associada ao número quântico magnético $-1 / 2(+1 / 2)$. 
Já o operador $\sigma_{k+1}^{+} \sigma_{k}^{-}$atua sobre estados do sistema, diminuindo a projeção do spin na direção $z$ por uma unidade, no sítio $k$. Posteriormente, o sítio vizinho $k+1$ tem sua projeção incrementada por uma unidade. A situação inversa é descrita pelo seu hermitiano conjugado. Neste formato, os operadores $\hat{\sigma}_{k+1}^{+} \hat{\sigma}_{k}^{-}$e seu hermitiano conjugado explicitam a difusão de spins pela cadeia, enquanto acoplamentos contendo $n_{k}$ férmions descrevem barreiras de potencial para a mencionada difusão.

Considerando-se a difusão e potenciais estáticos, bem como translações, o hamiltoniano (5.10) assume a seguinte forma

$$
\hat{H}_{\mathrm{sc}}=-J \frac{\hbar^{2}}{2} \sum_{k=1}^{L}\left(\hat{\sigma}_{k+1}^{+} \hat{\sigma}_{k}^{-}+\hat{\sigma}_{k}^{+} \hat{\sigma}_{k+1}^{-}+2 \Delta \hat{n}_{k} \hat{n}_{k+1}-2 \Delta \hat{n}_{k}\right)-J \frac{\hbar^{2}}{4} \Delta L .
$$

O operador número total de partículas em cada cadeia, $\hat{N}=\sum_{k} \hat{n}_{k}$, relaciona-se com o operador magnetização $\hat{M}=\sum_{k} \hat{\sigma}_{k}^{z}=2 \hat{N}-L$. Além disso, o operador expresso na Eq. (5.11) conserva a magnetização e, por extensão, ocorre também a conservação do número de partículas, ou seja, $\left[\hat{N}, \hat{H}_{\mathrm{sc}}\right]=0$. Adicionalmente, a conjunção da conservação de $N$ com a invariância translacional fixa o valor esperado da ocupação por sítio $\langle n\rangle=N / L$.

Salienta-se que o operador na Eq. (5.11) possui um rico espectro de energia e caracteriza os fenômenos coletivos que geram a disputa ordem/desordem. Para campo magnético $h$ nulo e $-1<\Delta<1$, o operador Eq. (5.11) possui o seguinte espectro [32]:

$$
E_{m m^{\prime}}^{\alpha \beta}-E_{0}=\frac{2 \pi v}{L} x_{m m^{\prime}}^{\alpha \beta}+o\left(L^{-2}\right) .
$$

A velocidade do som $v=v(\Delta)$ caracteriza a propagação da excitação e é obtida a partir da primeira excitação no momento; a dimensão anômala $x_{m m^{\prime}}^{\alpha \beta}=x^{\alpha \beta}+m+m^{\prime}$ determina a degenerescência e posição do nível no setor de momento $\alpha$, magnetização $\beta$, com $m, m^{\prime}$ inteiros positivos. O parâmetro $x^{\alpha \beta}$ caracteriza as correlações espaciais da excitação. A energia do estado fundamental,

$$
E_{0}=e_{\infty}-\frac{\pi v c}{6 L}+o\left(L^{-2}\right)
$$

possui a assinatura da anomalia conforme ${ }^{1} c$, que caracteriza a classe de universalidade do sistema [35]. Em particular, o valor para condições de contorno não

\footnotetext{
${ }^{1} \mathrm{~A}$ anomalia ou carga conforme é oriunda das leis de escala do operador energia-momentum sob a óptica de simetrias conformes em duas dimensões. Seu valor independe dos detalhes microscópicos da rede adjacente e caracteriza de maneira única as diversas classes de universalidade. Como exemplo, o modelo isotrópico XY possui $c=1$, enquanto Ising bidimensional possui $c=1 / 2$.
} 
torcidas $^{2}$ é $c=1$. O primeiro termo do lado direito da expressão acima, $e_{\infty}$, é a energia no limite termodinâmico e depende das características microscópicas do sistema estudado. Esta descrição para o espectro de energia é típico para cadeias com relação de dispersão relativística. De fato, a forma acima é consequência da associação entre cadeias unidimensionais de spin com Teorias de Campo Conforme em $1+1$ dimensões.

\subsection{Fermionização}

Há ainda um aspecto importante a ser discutido sobre a natureza dos operadores de spin localizados, $\hat{\sigma}_{k}^{ \pm}$. Para $k>k^{\prime}$, sem perda de generalidade, suas relações de comutação $\left[\hat{\sigma}_{k}^{ \pm}, \hat{\sigma}_{k^{\prime}}^{ \pm}\right]=0,\left[\hat{\sigma}_{k}^{ \pm}, \hat{\sigma}_{k^{\prime}}^{\mp}\right]=0$; enquanto a relação de anticomutação no mesmo sítio é $\left\{\hat{\sigma}_{k}^{ \pm}, \hat{\sigma}_{k}^{\mp}\right\}=1$. Estas relações algébricas não determinam se tais operadores são fermiônicos ou bosônicos. Essa incongruência é eliminada com o procedimento conhecido por fermionização ${ }^{3}$. O processo consiste na aplicação de transformações inversíveis $W_{k}$ sobre os operadores de levantamento e abaixamento, tal que as relações de anticomutação sejam aquelas tipicamente associadas aos férmions. Para isso, define-se um novo conjunto de operadores de criação e destruição, $\hat{c}_{k}^{\dagger}$ e $\hat{c}_{k}$, localizados no sítio $k$, que satisfazem às seguintes regras

$$
\left\{\hat{c}_{k}^{\dagger}, \hat{c}_{k^{\prime}}\right\}=\delta_{k k^{\prime}}, \quad\left\{\hat{c}_{k}, \hat{c}_{k^{\prime}}\right\}=0 \text {. }
$$

Cabe às transformações unitárias $\hat{W}_{k}$ a introdução de correlações não locais a fim de satisfazer as propriedades mencionadas acima. O método tradicional para a construção de $\hat{W}_{k}$ é realizado da seguinte maneira [10]

$$
\hat{W}_{k}=\exp \left(i \pi \sum_{l=1}^{k-1} \hat{n}_{l}\right) .
$$

Nota-se que essa escolha satisfaz o papel imposto às transformações $\hat{W}_{k}$, garantindo a não localidade da fase, tal que $\hat{c}_{k}=\hat{W}_{k} \hat{\sigma}_{k}^{-}$. Assim posta, as transformações $\hat{W}_{k}$ são conhecidas como transformações de Jordan-Wigner ou fermionização. A

\footnotetext{
${ }^{2}$ Condições de contorno torcidas ou twisted são aquelas onde há um ganho de fase $\varphi$ não múltiplo de $\pi$ sempre que o contorno é atingido. Neste caso, a fase do contorno é absorvida pela anomalia conforme, $c(\varphi)=c_{0}+f(\varphi)$, onde $f(\varphi)$ é uma função que depende das interações e simetrias presentes. Como $c(\varphi)$ define classes de universalidade, o ajuste de $\varphi$ permite a exploração de diversos comportamentos críticos em um único sistema.

${ }^{3}$ Procedimento análogo conhecido como bosonificação também pode ser realizado. Neste caso, as propriedades bosônicas emergem e dão origem à interpretação de operadores das excitações elementares. Ainda assim, o papel do potencial químico deve ser cuidadosamente analisado já que os níveis de energia e, portanto, ocupações de excitações são limitados.
} 
partir destas definições, mostra-se que o operador número adota a forma $\hat{n}_{k}=\hat{c}_{k}^{\dagger} \hat{c}_{k}$. Adicionalmente, também determina-se as seguintes propriedades, a saber,

$$
\left\{\hat{c}_{k}^{\dagger}, \hat{n}_{k}\right\}=\hat{c}_{k}^{\dagger}, \quad\left\{\hat{c}_{k}^{\dagger}, \mathrm{e}^{ \pm i \pi \hat{n}_{k}}\right\}=0, \quad\left[\hat{c}_{k}^{\dagger}, \hat{n}_{k^{\prime}}\right]=\delta_{k k^{\prime}} \hat{c}_{k}^{\dagger} \mathrm{e}^{i \pi\left(\hat{n}_{k}+1\right)} .
$$

Do processo de fermionização decorre também a alteração da condição de contorno da cadeia. Ela pode se manter periódica ou mudar para antiperiódica dependendo unicamente da paridade do número total de férmions $N$. A investigação do comportamento dos operadores de spin, no contorno, mostra que

$$
\hat{\sigma}_{L}^{\dagger} \hat{\sigma}_{1}^{-}+\mathrm{hc}=\mathrm{e}^{i \pi(N+1)} \hat{c}_{L}^{\dagger} \hat{c}_{1}+\mathrm{hc} .
$$

Devido à conservação de número de férmions de $\hat{H}_{\mathrm{sc}}$ na Eq. (5.11), o autovalor de $\mathrm{e}^{i \pi(N+1)}$ é constante em um dado setor de magnetização. Logo, essa fase pode ser absorvida na definição dos operadores $\hat{c}_{k}$ tal que

$$
\hat{c}_{k} \rightarrow \hat{c}_{k} \mathrm{e}^{-i k \gamma},
$$

$\operatorname{com} \gamma=-\pi(N+1) / L$. Essa transformação de gauge de primeira ordem reintroduz o contorno periódico e é válida para condições de contorno twisted em geral. As relações de anticomutação permanecem inalteradas enquanto os termos associados à energia cinética passam a carregar uma fase proporcional a magnetização local, isto é, $\hat{c}_{k+1}^{\dagger} \hat{c}_{k} \rightarrow \mathrm{e}^{i \gamma} \hat{c}_{k+1}^{\dagger} \hat{c}_{k}$. Já a energia potencial, $V=-J \Delta \hbar^{2} \sum_{k}\left(\hat{n}_{k} \hat{n}_{k+1}-\hat{n}_{k}\right)$, não sofre correções adicionais.

Uma vez realizada a fermionização, o operador $\hat{H}_{\mathrm{sc}}$ assume a seguinte forma:

$$
\hat{H}_{\mathrm{sc}}=-J\left(\hbar^{2} / 2\right) \sum_{k=1}^{L}\left(\hat{c}_{k+1}^{\dagger} \hat{c}_{k} \mathrm{e}^{i \gamma}+\hat{c}_{k}^{\dagger} \hat{c}_{k+1} \mathrm{e}^{-i \gamma}+2 \Delta n_{k+1} \hat{n}_{k}-2 \Delta \hat{n}_{k}\right) .
$$

Existem diversos métodos a extração de seus autoestados e respectivos autovalores ${ }^{4}$. A invariância por translações espaciais sugere a decomposição espectral

$$
\hat{c}_{k}=\frac{1}{\sqrt{2 \pi}} \int d q \sqrt{\rho(q, \Delta)} \mathrm{e}^{-i q r} \delta(r-k a) \hat{\eta}_{q}
$$

onde a densidade de estados $\rho(q, \Delta) \equiv \rho(q)$ é uma função real do acoplamento $\Delta$ e os operadores fermiônicos $\hat{\eta}_{q}^{\dagger}\left(\hat{\eta}_{q}\right)$ de criação(destruição) de partículas com momento $q$.

\footnotetext{
${ }^{4}$ Dentre os métodos mais conhecidos, três destacam-se: a decomposição espectral de Fourier, o ansatz de Bethe $[34,74]$ e o método de espalhamento inverso. Neste estudo abordamos a decomposição de Fourier. O ansatz de Bethe compartilha diversas similaridades com o método de Fourier. A principal diferença entre os dois métodos mencionados é que o ansatz assume uma distribuição não uniforme para os números de onda, os quais são obtidos mediante equações de autoconsistência. Por fim, o espalhamento inverso é fundamentado sobre o ansatz de Bethe, diferindo na metodologia para a extração das equações de autoconsistência.
} 
Em particular, para $\Delta=0$, reobtemos o caso livre, $\sqrt{\rho(q, 0)}=\sqrt{2 \pi / L} \sum_{m=0}^{L-1} \delta(q-$ $q_{m}$ ), onde $q_{m}$ é o $m$-ésimo momento da transformada de Fourier associada à cadeia de spin, isto é,

$$
\hat{c}_{k}=\frac{1}{\sqrt{L}} \sum_{m=0}^{L-1} \mathrm{e}^{i q_{m} k} \hat{\eta}_{q_{m}}
$$

Uma vez aplicada as transformações da Eq. (5.20) no operador $\hat{H}_{\mathrm{sc}}$ da Eq. (5.19), obtém-se

$$
\begin{aligned}
& \frac{\hat{H}_{\mathrm{sc}}}{-J \hbar^{2} / 2}=\int d q\left(\frac{L}{2 \pi}\right)\left[2 \cos (q+\gamma)+2 \Delta\left(\frac{\rho(q)}{2 \pi}-1\right)\right] \rho(q) \hat{\eta}_{q}^{\dagger} \hat{\eta}_{q}+ \\
& -\frac{\Delta L}{2 \pi^{2}} \int d^{3} q\left[\rho\left(q_{1}\right) \rho\left(q_{2}\right) \rho\left(q_{3}\right) \rho\left(q_{1}+q_{3}-q_{2}\right)\right]^{1 / 2} \hat{\eta}_{q_{1}}^{\dagger} \hat{\eta}_{q_{3}}^{\dagger} \hat{\eta}_{q_{2}} \hat{\eta}_{q_{1}+q_{3}-q_{2}} .
\end{aligned}
$$

O conhecimento da distribuição dos números de onda $\rho(q)$ permite a execução do cálculo do espectro de energia de $\hat{H}_{\mathrm{sc}}$, bem como efeitos de correlação e ordenamento. Por outro lado, o conhecimento preciso de $\rho(q)$ é equivalente a determinação exata dos autoestados do problema interagente. Para o caso livre, $\Delta=0$, temos a familiar expressão

$$
\frac{\hat{H}_{\mathrm{sc}}}{J \hbar^{2} / 2}=-\sum_{m=0}^{L-1} 2 \cos \left(q_{m}+\gamma\right) \hat{\eta}_{q_{m}}^{\dagger} \hat{\eta}_{q_{m}},
$$

com os números de onda dados por $q_{m}=-\pi+2 \pi m / L,(m=0,1, \ldots, L-1)$. Um exemplo muito simples é obtido para uma cadeia $L=4$ e $N=2$ para $\Delta=0$. Em termos da zona de Brillouin, os números de onda possíveis são $Q \in\{-3 \pi / 4,-\pi / 4, \pi / 4,3 \pi / 4\}$. Define-se o setor de momento $P=\sum Q$ associado à translação espacial com energia $2 E^{N, P} / J \hbar^{2}=-2 \sum \cos (Q)$. O estado de menor energia para $(N, P)=(2,0)$ possui ocupação não nula para $Q_{1}=\pi / 4 \mathrm{e}$ $Q_{-1}=-\pi / 4$, tal que $2 E^{2,0} / J \hbar^{2}=-4 \cos (\pi / 4)$.

\subsection{Modelo}

Para cadeias acopladas, o cenário previamente apresentado modifica-se caso exista a troca de excitações elementares entre elas pois há quebra da simetria local $U(1)$, isto é, a conservação de magnetização local. Interpreta-se esse efeito como uma corrente de spin, que flui de uma cadeia para a outra, onde o meio atua como uma barreira permissível [166, 170]. Aqui, estamos interessados em estudar a dinâmica microscópica de duas cadeias de spin interagentes finitas, cujas dimensões são compatíveis com aquelas de sistemas nanoestruturados. 
O sistema é composto por duas cadeias lineares de spin $R_{1}$ e $R_{2}$, concêntricas e paralelas, com condições periódicas de contorno, separadas por um meio dielétrico de espessura $d$. Cada cadeia contém partículas de spin $1 / 2$ localizadas em sítios, as quais interagem com seus vizinhos próximos. $R_{1}$ e $R_{2}$ possuem espaçamento de rede $a_{1}=a_{2}=a$ com $L_{1}=L_{2}=L$ número de sítios. $R_{1}$ encontra-se em $z=-d / 2$, enquanto $R_{2}$ localiza-se em $z=d / 2$. A evolução do sistema é governada pelo seguinte operador hamiltoniano independente do tempo: $\hat{H}=\hat{H}_{1}+\hat{H}_{2}+\lambda \hat{H}_{12}$. Os operadores $\hat{H}_{1}$ e $\hat{H}_{2}$ descrevem as interações locais entre partículas contidas em $R_{1}$ e $R_{2}$, respectivamente. O conteúdo operatorial tanto de $\hat{H}_{1}$ quanto de $\hat{H}_{2}$ é descrito pelo operador (5.19) sem a transformação de gauge $(\gamma=0)$, com termo de contorno expresso pela Eq. (5.17). O operador $\hat{H}_{12}$ descreve as interações entre as cadeias, caracterizada pelo acoplamento $\lambda$.

Neste ponto, cabe retornar à questão das relações de anticomutação fermiônicas, mas desta vez entre os entes de cadeias distintas. Indicamos os operadores fermiônicos $\hat{c}_{i k}$ como operadores de destruição de férmions sem spin no sítio $k$ da cadeia $R_{i}(i=1,2)$. Analogamente, para os operadores de criação $\hat{c}_{i k}^{\dagger}$. Todos os operadores e parâmetros relevantes adotam a mesma nomenclatura, inclusive $\hat{n}_{i k}=\hat{c}_{i k}^{\dagger} \hat{c}_{i k}$, que é o operador número do $k$-ésimo sítio da rede $R_{i}$. De acordo com a argumentação feita na seção anterior, dois objetos são férmions se $\left\{\hat{c}_{i k}, \hat{c}_{i^{\prime} k^{\prime}}^{\dagger}\right\}=\delta_{i^{\prime} i} \delta_{k^{\prime} k}$. Posto que os férmions em $R_{1,2}$ são construídos de maneira independente, fica patente que a relação de anticomutação fermiônica não é válida. Ao contrário, espera-se que comutem, ou seja, $\left[\hat{c}_{i k}, \hat{c}_{i^{\prime} k^{\prime}}^{\dagger}\right]=0$ para $i \neq i^{\prime}$. Novamente, para que as relações fermiônicas sejam satisfeitas simultaneamente por ambas as cadeias, é necessário que também exista correlação entre férmions de cadeias distintas.

Para sanar essa dificuldade, implementamos uma segunda rodada de transformações fermiônicas. Uma escolha conveniente para a execução da fermionização é dada pela seguinte transformação:

$$
\hat{c}_{i k} \rightarrow \mathrm{e}^{i \hat{\varphi}_{i}} \hat{c}_{i k}, \quad \hat{c}_{i k}^{\dagger} \rightarrow \mathrm{e}^{-i \hat{\varphi}_{i}} \hat{c}_{i k}^{\dagger}
$$

O operador $\hat{\varphi}_{i}$ introduz as correlações não-locais entre as diferentes cadeias. Escolhemos convenientemente $\hat{\varphi}_{1}=0$ e $\hat{\varphi}_{2}=\pi \sum_{l=1}^{L} \hat{n}_{1 l}=\pi \hat{N}_{1}$. Quando não há interação entre as cadeias, $\hat{\varphi}_{1,2}$ nada mais são do que fases globais. De fato, a introdução destas fases deve-se somente ao requerimento de que ambos sejam férmions e cuja 
origem é puramente algébrica. Produtos bilineares naturalmente cancelam tais fases. Isto significa que a correlação entre férmions de $R_{1}$ e $R_{2}$ não sofre redução com o incremento da separação espacial.

O operador $\lambda \hat{H}_{12}$ contém os ingredientes necessários para descrever a ocorrência de eventos de tunelamento de spin entre as cadeias $R_{1}$ e $R_{2}$. Neste caso, o operador mais geral é descrito como

$$
\frac{\lambda \hat{H}_{12}}{J \hbar^{2} / 2}=\sum_{k, k^{\prime}} \lambda_{k k^{\prime}}^{\mathrm{eff}}\left(\hat{c}_{1 k}^{\dagger} \hat{c}_{2 k^{\prime}}+\mathrm{hc}\right)+2 \Delta^{\mathrm{eff}} \sum_{k}\left(\hat{n}_{1 k}-\hat{n}_{2 k}\right) .
$$

O acoplamento $\lambda_{k k^{\prime}}^{\text {eff }}$ depende das características microscópicas e temperatura. Já o acoplamento $\Delta^{\text {eff }}$ é responsável pelo equilíbrio magnetização. Claramente, a generalidade do hamiltoniano na Eq. (5.25) carece de restrições para que seja possível realizar um tratamento analítico. A seguir, impomos as restrições ao modelo e os critérios para seu estabelecimento.

As interações contidas em $\lambda \hat{H}_{12}$ quebram a conservação local da magnetização. Isto é facilmente observado pela presença dos operadores $\hat{c}_{1 k}^{\dagger} \hat{c}_{2 k^{\prime}}$. Estes removem spins de $R_{2}$ e os transferem para $R_{1}$. Seu hermitiano conjugado realiza a operação inversa. Porém a conservação do número total de férmions, $N=\sum_{j, k} n_{j k}$, ainda permanece uma quantidade conservada $\left[\lambda \hat{H}_{12}, \hat{N}\right]=0$.

Aqui considera-se a dinâmica mais simples que contemple tunelamentos e satisfaça as conservações mencionadas acima, ou seja, $\lambda_{k^{\prime} k}^{\text {eff }}=\lambda \delta_{k^{\prime} k}$. O acoplamento diagonal $\lambda$ captura efeitos que influenciam inversão de população de modos com velocidade baixa. Considerando-se essas modificações, o hamiltoniano assume a forma

$$
\begin{aligned}
\frac{\hat{H}_{\mathrm{eff}}}{J \hbar^{2} / 2}= & -\sum_{i=1}^{2} \sum_{k=1}^{L-1}\left(\hat{c}_{i k+1}^{\dagger} \hat{c}_{i k}+\hat{c}_{i k}^{\dagger} \hat{c}_{i k+1}+2 \Delta_{i} \hat{n}_{i k+1} \hat{n}_{i k}\right)+ \\
& +\sum_{k}^{L}\left(2 \Delta_{1}^{\prime} \hat{n}_{1 k}+2 \Delta_{2}^{\prime} \hat{n}_{2 k}+\lambda \hat{c}_{1 k}^{\dagger} \hat{c}_{2 k}+\lambda \hat{c}_{2 k}^{\dagger} \hat{c}_{1 k}\right)+ \\
& -\sum_{i}\left(\mathrm{e}^{i \pi\left(\hat{N}_{i}+1\right)} \hat{c}_{i L}^{\dagger} \hat{c}_{i 1}+\text { hc } .\right)
\end{aligned}
$$

com $\Delta_{1}^{\prime}=\Delta_{1}+\Delta^{\text {eff }}$ e $\Delta_{2}^{\prime}=\Delta_{2}-\Delta^{\text {eff }}$. Ao contrário do modelo livre $\mathrm{XY}$, as cadeias podem desenvolver ordenamento mesmo com ausência de um eixo preferencial de magnetização, isto é, em $\Delta_{i}=0$. 


\subsection{Quasipartículas}

O operador hamiltoniano da Eq. (5.26) contém as interações que descrevem a dinâmica de férmions interagentes sem spin. Mais ainda, apresenta simetria por reflexão em $z=0$. Quando em conjunto com a invariância translacional, há degenerescência das energias a respeito da definição do sentido do movimento entre as cadeias ou helicidade. Outro aspecto importante a ser revisado é a fase na Eq. (5.24) que os operadores $c_{2 k}$ carregam sobre $R_{1}$. Essas questões servem de elemento motivador para a formulação de transformações canônicas capazes de responder às incongruências acima sem perturbar o cenário de formulação fermiônica.

Ao invés de trabalhar com férmions sem spin $\hat{c}_{1 k}$ e $\hat{c}_{2 k}$, define-se um novo conjunto localizado de quasipartículas $\hat{\psi}_{k \uparrow}$ e $\hat{\psi}_{k \downarrow}$,

$$
\begin{aligned}
& \hat{\psi}_{k \uparrow}^{\dagger}=+\cos \theta_{k} \hat{c}_{1 k}^{\dagger}+\sin \theta_{k} \hat{c}_{2 k}, \quad \hat{\psi}_{k \uparrow}=+\cos \theta_{k} \hat{c}_{1 k}+\sin \theta_{k} \hat{c}_{2 k}^{\dagger}, \\
& \hat{\psi}_{k \downarrow}^{\dagger}=-\cos \theta_{k} \hat{c}_{2 k}^{\dagger}+\sin \theta_{k} \hat{c}_{1 k}, \quad \hat{\psi}_{k \downarrow}=-\cos \theta_{k} \hat{c}_{2 k}+\sin \theta_{k} \hat{c}_{1 k}^{\dagger} .
\end{aligned}
$$

Uma notável consequência é a manutenção do número de graus de liberdade, porém, com redução efetiva da dimensionalidade do sistema e um grau de liberdade adicional $\sigma=\uparrow, \downarrow$. Por conveniência, associa-se o grau de liberdade $\sigma$ com pseudo-spin. A álgebra que os operadores de criação e destruição das novas quasipartículas satisfazem são aquelas de férmions, a saber, $\left\{\hat{\psi}_{k \sigma}^{\dagger}, \hat{\psi}_{k^{\prime} \sigma^{\prime}}\right\}=\delta_{k^{\prime} k} \delta_{\sigma^{\prime} \sigma}$ e identicamente nula para todas as demais. Os parâmetros $\theta_{k}$ valores são ajustados de maneira fixar a dinâmica dos férmions $\hat{\psi}_{k \sigma}$, bem como condições de contorno e densidade de estados. Esta é uma propriedade inerente de sistemas fortemente correlacionados [171].

Ainda vale a pena observar que essa parametrização permite uma forte interpretação em termos de rotações $\mathrm{SU}(2)$ ao redor de um eixo,

$$
\left(\begin{array}{c}
\hat{\psi}_{k \uparrow}^{\dagger} \\
\hat{\psi}_{k \downarrow} \\
\hat{\psi}_{k \downarrow}^{\dagger} \\
\hat{\psi}_{k \uparrow}
\end{array}\right)=\left(\begin{array}{cccc}
\cos \theta_{k} & \sin \theta_{k} & 0 & 0 \\
\sin \theta_{k} & -\cos \theta_{k} & 0 & 0 \\
0 & 0 & \cos \theta_{k} & \sin \theta_{k} \\
0 & 0 & \sin \theta_{k} & -\cos \theta_{k}
\end{array}\right)\left(\begin{array}{c}
\hat{c}_{1 k}^{\dagger} \\
\hat{c}_{2 k} \\
\hat{c}_{2 k}^{\dagger} \\
\hat{c}_{1 k}
\end{array}\right) .
$$

Por ser uma rotação $\mathrm{SU}(2)$, podemos interpretar a quasipartícula como um ente de spin 1/2. A inversão é facilmente obtida (dado que cada bloco diagonal é uma 
matriz $2 \times 2$ ) e de onde se obtém

$$
\begin{aligned}
& \hat{c}_{1 k}^{\dagger}=\cos \theta_{k} \hat{\psi}_{k \uparrow}^{\dagger}+\sin \theta_{k} \hat{\psi}_{k \downarrow}, \\
& \hat{c}_{1 k}=\cos \theta_{k} \hat{\psi}_{k \uparrow}+\sin \theta_{k} \hat{\psi}_{k \downarrow}^{\dagger}, \\
& \hat{c}_{2 k}^{\dagger}=\sin \theta_{k} \hat{\psi}_{k \uparrow}-\cos \theta_{k} \hat{\psi}_{k \downarrow}^{\dagger}, \\
& \hat{c}_{2 k}=\sin \theta_{k} \hat{\psi}_{k \uparrow}^{\dagger}-\cos \theta_{k} \hat{\psi}_{k \downarrow} .
\end{aligned}
$$

É possível extrair uma propriedade relevante sobre as condições de contorno nesta nova formulação. Primeiro, a transformação que levam os operadores de levantamento $\hat{\sigma}_{i k}^{+}$em operadores fermiônicos $\hat{c}_{i k}^{\dagger}$ exigem $\hat{c}_{i L+1}^{\dagger}=\left(\mathrm{e}^{i \pi \sum_{l}^{L} \hat{n}_{i l}}\right) \hat{c}_{i 1}^{\dagger}$. Essa condição de auto-consistência no contorno conduz a um termo de superfície. A conservação local de número de partículas, em uma dada rede, permite que essa contribuição seja espalhada para todos os operadores via transformação de gauge de primeiro tipo, restaurando a condição periódica de contorno. O viés de tal transformação de gauge é a modificação na distribuição dos momenta, como observa-se na Eq. (5.18). Quando existe interação entre as cadeias, não fica claro a priori se é possível definir uma transformação de gauge pois também cessa a conservação local do número de partículas.

As transformações fermiônicas para partículas com spin $\hat{\psi}_{k \sigma}$ automaticamente resolvem a questão da condição de contorno. Isto se deve à liberdade dos parâmetros $\theta_{k}$. Escolhendo-se $\theta_{L+1}=\theta_{1}+\pi N=\theta_{1}+\pi N_{1}+\pi N_{2}$, obtém-se a seguinte regra de transformação para $\hat{c}_{1 L+1}$,

$$
\hat{c}_{1 L+1}=\cos \pi N_{1} \cos \left(\theta_{1}+\pi N_{2}\right) \hat{\psi}_{L+1 \uparrow}+\cos \pi N_{1} \sin \left(\theta_{1}+\pi N_{2}\right) \hat{\psi}_{L+1 \downarrow}^{\dagger}
$$

A associação $\hat{c}_{1 L+1}=\cos \left(\pi N_{1}\right) \hat{c}_{1}$ é consistente caso os operadores fermiônicos $\hat{\psi}_{k \sigma}$ transformem-se como $\hat{\psi}_{L+1 \sigma}=\hat{\psi}_{1 \sigma}$, para $\sigma=\downarrow$, $\uparrow$. Situação análoga ocorre para os operadores $\hat{c}_{2 L+1}$. Conclui-se, portanto, que a condição periódica é restaurada, independentemente do número local de partículas.

Com as condições de contorno bem definidas, podemos analisar com segurança as regras e condições de transformação dos operadores bilineares do hamiltoniano na Eq. (5.26). Uma breve inspeção das transformações das Eqs. (5.27a)-(5.27b) mostra que o produto bilinear $\hat{c}_{i k}^{\dagger} \hat{c}_{i k}$ produz termos diagonais e cruzados,

$$
\hat{c}_{1 k}^{\dagger} \hat{c}_{1 k}=\cos ^{2} \theta_{k} \hat{\psi}_{k \uparrow}^{\dagger} \hat{\psi}_{k \uparrow}+\sin ^{2} \theta_{k} \hat{\psi}_{k \downarrow} \hat{\psi}_{k \downarrow}^{\dagger}+\frac{\sin 2 \theta_{k}}{2}\left(\hat{\psi}_{k \uparrow}^{\dagger} \hat{\psi}_{k \downarrow}^{\dagger}-\hat{\psi}_{k \uparrow} \hat{\psi}_{k \downarrow}\right),
$$




$$
\hat{c}_{2 k}^{\dagger} \hat{c}_{2 k}=\cos ^{2} \theta_{k} \hat{\psi}_{k \downarrow}^{\dagger} \psi_{k \downarrow}+\sin ^{2} \theta_{k} \hat{\psi}_{k \uparrow} \hat{\psi}_{k \uparrow}^{\dagger}+\frac{\sin 2 \theta_{k}}{2}\left(\hat{\psi}_{k \uparrow}^{\dagger} \hat{\psi}_{k \downarrow}^{\dagger}-\hat{\psi}_{k \uparrow} \hat{\psi}_{k \downarrow}\right)
$$

Para simplificar a discussão, define-se o operador número para férmions $\hat{n}_{k \sigma}=$ $\hat{\psi}_{k \sigma}^{\dagger} \hat{\psi}_{k \sigma}$ com spin $\sigma=\uparrow, \downarrow$. Adicionalmente, define-se as seguintes quantidades

$$
\begin{aligned}
\bar{n}_{k \uparrow} & =\cos ^{2} \theta_{k} \hat{n}_{k \uparrow}-\sin ^{2} \theta_{k} \hat{n}_{k \downarrow}, \\
\bar{n}_{k \downarrow} & =\cos ^{2} \theta_{k} \hat{n}_{k \downarrow}-\sin ^{2} \theta_{k} \hat{n}_{k \uparrow}, \\
\hat{C}_{k} & =\sin ^{2} \theta_{k}+\frac{\sin 2 \theta_{k}}{2}\left(\hat{\psi}_{k \uparrow}^{\dagger} \hat{\psi}_{k \downarrow}^{\dagger}+\hat{\psi}_{k \downarrow} \hat{\psi}_{k \uparrow}\right) .
\end{aligned}
$$

O operador $\hat{C}_{k}$ contém os operadores associados a criação e destruição de pares de férmions num mesmo sítio. As Eqs. (5.31a) e (5.31b) produzem $\hat{n}_{1 k}-\hat{n}_{2 k}=\hat{n}_{k \uparrow}-\hat{n}_{k \downarrow}$, que é uma identidade local. A diferença de populações pode ser vista como um a projeção de spin no eixo de quantização, $\hat{Q}_{k}$, e relaciona-se com a magnetização vertical da nanoestrutura,

$$
\hat{Q}_{k}=\hat{n}_{k \uparrow}-\hat{n}_{k \downarrow}=\hat{n}_{1 k}-\hat{n}_{2 k} .
$$

Mais ainda, a conservação da magnetização total permite a seguinte regra de soma:

$$
\sum_{k=1}^{L} \hat{C}_{k}=\frac{N}{2}-\frac{1}{2} \sum_{k=1}^{L} \cos 2 \theta_{k}\left(\hat{n}_{k \uparrow}+\hat{n}_{k \downarrow}\right) .
$$

A partir deste resultado, ficam estabelecidos seus limites superior e inferior: $0 \leqslant$ $\left\langle\sum C_{k}\right\rangle \leqslant N$. A invariância translacional fornece outra propriedade importante, a saber, $\left\langle\sum C_{k}\right\rangle=\left\langle C_{k}\right\rangle L=N / 2-\left\langle\cos 2 \theta_{k}\left(\hat{n}_{k \uparrow}+\hat{n}_{k \downarrow}\right)\right\rangle L / 2$, isto é,

$$
\left\langle\sin 2 \theta_{k}\left(\hat{\psi}_{k \uparrow}^{\dagger} \hat{\psi}_{k \downarrow}^{\dagger}+\hat{\psi}_{k \downarrow} \hat{\psi}_{k \uparrow}\right)\right\rangle+\left\langle\cos 2 \theta_{k}\left(\hat{n}_{k \uparrow}+\hat{n}_{k \downarrow}-1\right)\right\rangle=\frac{N}{L}-1 .
$$

Para $N=L$, segue que $\left\langle\cos 2 \theta_{k}\left(\hat{n}_{k \uparrow}+\hat{n}_{k \downarrow}-1\right)\right\rangle \rightarrow 0$ implica na ausência de pares de férmions ou sítios desocupados. O grau de ocupação de um dado sítio fica então diretamente associado com a distribuição de valores dos ângulos $\theta_{k}$ e sua relação com a densidade total de férmions $N / L$.

Os operadores que registram o tunelamento de férmions entre as cadeias são obtidos a partir de $\hat{c}_{1 k}^{\dagger} \hat{c}_{2 k}+\hat{c}_{2 k}^{\dagger} \hat{c}_{1 k}=-\left(\hat{\psi}_{k \uparrow}^{\dagger} \hat{\psi}_{k \downarrow}+\hat{\psi}_{k \downarrow}^{\dagger} \hat{\psi}_{k \uparrow}\right)$. Ao invés de realizar a troca de partículas entre duas cadeias, o sistema unidimensional passa a flipar os spins dos férmions $\hat{\psi}_{k \sigma}$ com intensidade oposta àquela do modelo original. Em seguida, estuda-se as leis de transformação para os termos cinéticos. Estes são caracterizados 
por operadores $\hat{c}_{i k}^{\dagger} \hat{c}_{i k^{\prime}}$, onde uma partícula é destruída no sítio $k^{\prime}$ e criada em $k$ na cadeia $R_{i}$,

$$
\begin{aligned}
\hat{c}_{1 k}^{\dagger} \hat{c}_{1 l}+\hat{c}_{1 l}^{\dagger} \hat{c}_{1 k} & =\cos \theta_{k} \cos \theta_{l}\left(\hat{\psi}_{k \uparrow}^{\dagger} \hat{\psi}_{l \uparrow}+\hat{\psi}_{l \uparrow}^{\dagger} \hat{\psi}_{k \uparrow}\right)-\sin \theta_{k} \sin \theta_{l}\left(\hat{\psi}_{k \downarrow}^{\dagger} \hat{\psi}_{l \downarrow}+\hat{\psi}_{l \downarrow}^{\dagger} \hat{\psi}_{k \downarrow}\right) \\
& +\cos \theta_{k} \sin \theta_{l}\left(\hat{\psi}_{k \uparrow}^{\dagger} \hat{\psi}_{l \downarrow}^{\dagger}-\hat{\psi}_{k \uparrow} \hat{\psi}_{l \downarrow}\right)+\cos \theta_{l} \sin \theta_{k}\left(\hat{\psi}_{l \uparrow}^{\dagger} \hat{\psi}_{k \downarrow}^{\dagger}-\hat{\psi}_{l \uparrow} \hat{\psi}_{k \downarrow}\right) . \\
\hat{c}_{2 k}^{\dagger} \hat{c}_{2 l}+\hat{c}_{2 l}^{\dagger} \hat{c}_{2 k} & =\cos \theta_{k} \cos \theta_{l}\left(\hat{\psi}_{k \downarrow}^{\dagger} \hat{\psi}_{l \downarrow}+\hat{\psi}_{l \downarrow}^{\dagger} \hat{\psi}_{k \downarrow}\right)-\sin \theta_{k} \sin \theta_{l}\left(\hat{\psi}_{k \uparrow}^{\dagger} \hat{\psi}_{l \uparrow}+\hat{\psi}_{l \uparrow}^{\dagger} \hat{\psi}_{k \uparrow}\right) \\
& +\cos \theta_{k} \sin \theta_{l}\left(\hat{\psi}_{l \uparrow}^{\dagger} \hat{\psi}_{k \downarrow}^{\dagger}-\hat{\psi}_{l \uparrow} \hat{\psi}_{k \downarrow}\right)+\cos \theta_{l} \sin \theta_{k}\left(\hat{\psi}_{k \uparrow}^{\dagger} \hat{\psi}_{l \downarrow}^{\dagger}-\hat{\psi}_{k \uparrow} \hat{\psi}_{l \downarrow}\right) .
\end{aligned}
$$

As transformações acima preveem a possibilidade de criação e destruição de pares oriundos de um operador difusivo. Este cenário difere daquele de partículas difusivas confinadas numa rede. Para remover esse comportamento não-físico, vínculos são impostos à distribuição de valores dos ângulos $\theta_{k}$. A soma das expressões (5.36a) e (5.36b) determina a seguinte condição: $\sin \left(\theta_{k}+\theta_{k+1}\right)=0$. Logo, desde que essa condição seja satisfeita, a difusão dos $\hat{\psi}_{i \sigma}$ também conserva o número total de férmions. É importante salientar que a equação acima correlaciona os valores dos ângulos $\theta_{k}$ entre seus vizinhos. Por ser um sistema unidimensional efetivo, fixando-se o valor de $\theta_{1}$, em princípio, os valores de todos os outros $L-1$ parâmetros são fixados. Essa observação mostra que a paridade e comprimento total da cadeia, implicitamente, desempenham papel relevante para determinação do valor $\theta_{1}$. Nesta situação, o operador associado à difusão dos férmions $\hat{\psi}_{k \sigma}$,

$$
\left.\hat{c}_{1 k}^{\dagger} \hat{c}_{1 k+1}+\hat{c}_{2 k}^{\dagger} \hat{c}_{2 k+1}+\mathrm{hc}\right)=\cos \left(\theta_{k}+\theta_{k+1}\right)\left(\hat{\psi}_{k \uparrow}^{\dagger} \hat{\psi}_{k+1 \uparrow}-\hat{\psi}_{k \downarrow}^{\dagger} \hat{\psi}_{k+1 \downarrow}+\mathrm{hc}\right),
$$

mostra que contribuição para a difusão dos dois tipos de férmions possuem amplitude iguais, como na formulação anterior, mas opostas. Como discutido anteriormente, isto é um reflexo da degenerescência em relação à escolha do sentido do movimento entre ambas as cadeias.

Por fim, consideramos as regras e vínculos derivados dos operadores associados à energia potencial. Seja o operador número de férmions sem spin $\hat{n}_{k}=\hat{n}_{1 k}+\hat{n}_{2 k}$ do sítio $k$. A partir de $\hat{n}_{k}$ e do operador carga $\hat{Q}_{k}$, determina-se $\hat{n}_{1 k}=\left(\hat{n}_{k}+\hat{Q}_{k}\right) / 2$ e $\hat{n}_{2 k}=\left(\hat{n}_{k}-\hat{Q}_{k}\right) / 2$. Como vimos, $\hat{Q}_{k}=\hat{n}_{k \uparrow}-\hat{n}_{k \downarrow}$ enquanto $n_{k}=\cos 2 \theta_{k}\left(\hat{n}_{k \uparrow}+\hat{n}_{k \downarrow}\right)+2 \hat{C}_{k}$. O operador $\hat{C}_{k}$ por sua vez, contém operadores de criação e destruição de pares, modulados por $\sin 2 \theta_{k}$. Portanto, a soma dos potenciais 
estáticos de contato, $\hat{n}_{i k} \hat{n}_{i k+1}$, é escrita como

$$
\sum_{i=1,2} \Delta_{i} \hat{n}_{i k} \hat{n}_{i k+1}=\left(\frac{\Delta_{1}+\Delta_{2}}{2}\right)\left(\hat{n}_{k} \hat{n}_{k+1}+\hat{Q}_{k} \hat{Q}_{k+1}\right)+\left(\frac{\Delta_{1}-\Delta_{2}}{2}\right)\left(\hat{Q}_{k} \hat{n}_{k+1}+\hat{n}_{k} \hat{Q}_{k+1}\right) .
$$

As interações quadráticas representam um grande desafio para a resolução exata do problema. Essa interação pode ser simplificada pela introdução da aproximação de campo médio, $\sum_{i, k} \Delta_{i} \hat{n}_{i k} \hat{n}_{i k+1} \approx F_{+} \hat{N}+F_{-} \sum_{k} \hat{Q}_{k}$. Aqui, $\Delta_{ \pm}=\left(\Delta_{1} \pm \Delta_{2}\right) / 2 \mathrm{e}$ $F_{ \pm}=\left(\Delta_{ \pm}\langle n\rangle+\Delta_{\mp}\langle Q\rangle\right) / 2$, com $\langle n\rangle=L^{-1} \sum\left\langle n_{k}\right\rangle$ e $\langle Q\rangle=L^{-1} \sum\left\langle Q_{k}\right\rangle$. O cenário das quasipartículas mantém correlações, mesmo na aproximação de campo médio, enquanto $\langle Q\rangle \Delta_{+} \neq-\langle n\rangle \Delta_{-}$. No que segue, admite-se que $F_{-} \neq 0$.

\subsection{Hamiltoniano canônico}

A dinâmica existente neste nanosistema com tunelamento entre cadeias de spin caracteriza-se por três acoplamentos: $\Delta_{1}, \Delta_{2}$ e $\lambda$. O caso mais simples, que mimica partículas livres, ocorre quando $\Delta_{1,2}=\lambda=0$. Este regime possui um comportamento crítico, o qual se mantém mesmo para valores não nulos de $\Delta_{1,2}$. Nesta seção, estudamos a modificação do comportamento crítico no cenário das quasipartículas $\psi_{k \sigma}$. Uma vez compreendidas as propriedades físicas da nanoestrutura, habilitamos o tunelamento, $\lambda \neq 0$, e analisamos as modificações pertinentes à nova dinâmica fermiônica.

Para $\lambda=0$, operador hamiltoniano é

$$
\hat{H}=-\sum_{k}\left[J \cos \left(\theta_{k}+\theta_{k+1}\right)-\varepsilon_{0}\right]\left(\hat{\psi}_{k \uparrow}^{\dagger} \hat{\psi}_{k+1 \uparrow}-\hat{\psi}_{k \downarrow}^{\dagger} \hat{\psi}_{k+1 \downarrow}+\mathrm{hc}\right)+H_{N},
$$

onde $\varepsilon_{0}=-2 J\left(F_{-} \Delta_{)}\right.$e $H_{N}=-2 J\left(F_{+}-\Delta_{+}\right) N$. A decomposição espectral $\psi_{k \sigma}=$ $L^{-1 / 2} \sum_{q} \mathrm{e}^{i q k} \Psi_{q \sigma}$, onde $\Psi_{q \sigma}$ é o operador de destruição de férmions de spin $\sigma$ e momento $q=-\pi+\pi / L+2 \pi m / L$ com $m=0, \ldots, L-1$, produz

$$
\hat{H}=\sum_{q, q^{\prime}}\left(\Omega_{q q^{\prime}}+\varepsilon_{0}\right)\left(\hat{\Psi}_{q \uparrow}^{\dagger} \hat{\Psi}_{q^{\prime} \uparrow}-\hat{\Psi}_{q \downarrow}^{\dagger} \hat{\Psi}_{q^{\prime} \downarrow}\right)+H_{N}
$$

$\operatorname{com} \Omega_{q q^{\prime}}=-(2 J \cos q / L) \sum_{k} \mathrm{e}^{i k\left(q-q^{\prime}\right)} \cos \left(\theta_{k}+\theta_{k+1}\right)$, onde $\theta_{k}+\theta_{k+1}=m_{k} \pi$ e $\theta_{L+1}=$ $\theta_{1}+\left(N_{1}+N_{2}\right) \pi$. A particular distribuição dos inteiros $m_{k}$ determina o valor de $\Omega_{q q^{\prime}}$. Aqui, assumimos que $\theta_{k}+\theta_{k+1}=2 m_{k} \pi$, que produz $\tilde{\Omega}_{q q^{\prime}}=-2 J \delta_{q q^{\prime}} \cos q$. As autoenergias do hamiltoniano na Eq. (5.40) ficam então determinada a partir da 
distribuição dos autovalores do operador número:

$$
E=\sum_{q}\left(-2 J \cos q+\varepsilon_{0}\right)\left(n_{q \uparrow}-n_{q \downarrow}\right)+H_{N} .
$$

A energia do estado fundamental, $E_{0}$, é obtida considerando-se máxima ocupação dos níveis que produzem $\cos q<0$, pelos férmions com $\sigma=\uparrow$. Já os férmions $\operatorname{com} \sigma=\downarrow$ apresentam $\cos q>0$. Mais ainda, o parâmetro de campo médio $\varepsilon_{0}$ desloca os níveis de energia para ambas as variedades de spin, mas em sentidos opostos. Quando $\varepsilon_{0}<0$, as energias de férmions com spin $\sigma=\uparrow(\sigma=\downarrow)$ são decrementadas (incrementadas), semelhante ao efeito Zeeman. Resultados numéricos são exibidos na Figura 5.1. Para o caso não-interagente, $\lambda=\Delta_{1,2}=0$ e a energia do estado fundamental é $\lim _{\Delta_{1,2} \rightarrow 0} E_{0}=-4 J / \sin (\pi / L)$. A partir deste resultado, torna-se possível extrair tanto o valor da energia no limite termodinâmico, $e_{\infty}=-4 \mathrm{JL} / \pi$, quanto a anomalia conforme $c=1$, que é a classe de universalidade do modelo XY unidimensional. A energia do estado excitado é obtida através da promoção de uma quasipartícula de momento $q=\pi / 2-\pi / L$ com $\sigma=\uparrow$ para o nível com momento $q^{\prime}=q+2 \pi / L: E_{1}=E_{0}+4 J \sin (\pi / L)$. Esse processo mantém tanto o número $N$ de férmions constante bem como a carga total $Q=\sum Q_{q}$. A expansão da energia de excitação expressa-se como uma relação de dispersão relativística, linear com o vetor de onda ou momento, $\Delta E_{1} \approx 2 J(2 \pi / L)=2 J(\delta q)$, donde se conclui que a velocidade da excitação é $v_{s}=2 a J$.

Cabe observar como o sistema se comporta quando contempla eventos de tunelamento, $\lambda \neq 0$, e além disso possui eixos preferencias de magnetização, $\Delta_{i} \neq 0$. Nessa situação, o hamiltoniano na aproximação de campo médio é

$$
\hat{H}=\sum_{q}\left(\tilde{\Omega}_{q q}+\varepsilon_{0}\right)\left(\hat{\Psi}_{q \uparrow}^{\dagger} \hat{\Psi}_{q \uparrow}-\hat{\Psi}_{q \downarrow}^{\dagger} \hat{\Psi}_{q \downarrow}\right)+\lambda \sum_{q}\left(\hat{\Psi}_{q \uparrow}^{\dagger} \hat{\Psi}_{q \downarrow}+\hat{\Psi}_{q \downarrow}^{\dagger} \hat{\Psi}_{q \uparrow}\right)+H_{N},
$$

onde $\varepsilon_{0}=-2 J\left(F_{-} \Delta_{)}\right.$e $H_{N}=-2 J\left(F_{+}-\Delta_{+}\right) N$. Para um dado momento $q$, o acoplamento $\lambda$ na Eq. (5.42) conecta dois apenas spins opostos, que sugere o emprego das transformações de Bogoliubov [12],

$$
\begin{aligned}
& \hat{\zeta}_{q \uparrow}=\cos \left(\frac{\alpha_{q}}{2}\right) \hat{\psi}_{q \uparrow}+\sin \left(\frac{\alpha_{q}}{2}\right) \hat{\psi}_{q \downarrow}, \\
& \hat{\zeta}_{q \downarrow}=\sin \left(\frac{\alpha_{q}}{2}\right) \hat{\psi}_{q \uparrow}-\cos \left(\frac{\alpha_{q}}{2}\right) \hat{\psi}_{q \downarrow}
\end{aligned}
$$

com $\tan \alpha_{q}=\lambda /\left(\tilde{\Omega}_{q q}+\varepsilon_{0}\right)$. Estes operadores, por sua vez, satisfazem as relações de anticomutação fermiônicas $\left\{\hat{\zeta}_{q \sigma}^{\dagger}, \hat{\zeta}_{q^{\prime} \sigma^{\prime}}\right\}=\delta_{q q^{\prime}} \delta_{\sigma \sigma^{\prime}}$. Os modos fermiônicos $\hat{\zeta}_{q \sigma}$ são 
modos coletivos resultantes do espalhamento entre as quasipartículas $\hat{\psi}_{q \sigma}$ com spins opostos. Utilizando os operadores diagonais $\zeta_{q \sigma}$, obtém-se a seguinte representação para o operador hamiltoniano

$$
\hat{H}=\sum_{q} \Lambda_{q}\left(\hat{\nu}_{q \uparrow}-\hat{\nu}_{q \downarrow}\right)+H_{N}
$$

onde $\nu_{q \sigma}=\zeta_{q \sigma}^{\dagger} \zeta_{q \sigma}$ é o operador número do modo $\zeta_{q \sigma} \operatorname{com} \Lambda_{q}=\lambda \cot \alpha_{q} \sqrt{1+\tan ^{2} \alpha_{q}}$.

Seja $X=X\left(\varepsilon_{0}\right)$ tal que $\Lambda_{X}=0$. Observa-se que os modos associados ao spin $\sigma=\uparrow$, com momento $-X \leqslant q \leqslant X$, fornecem contribuições negativas à energia. Para modos associados aos spins $\sigma=\downarrow$, ocorre a situação inversa: as contribuições negativas ocorrem somente para momento fora do intervalo $[-X, X]$. Os modos que produzem contribuições positivas geram níveis de alta energia, resultado da excitação do sistema. Esse sistema possui uma estrutura de bandas caracterizada

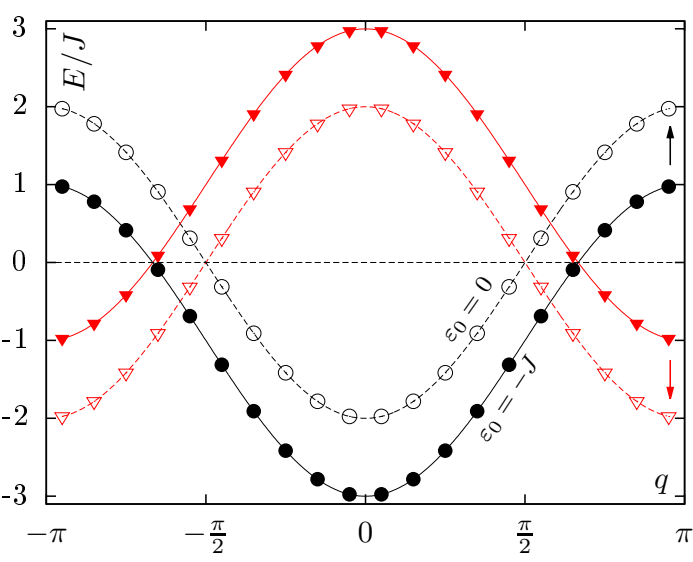

a)

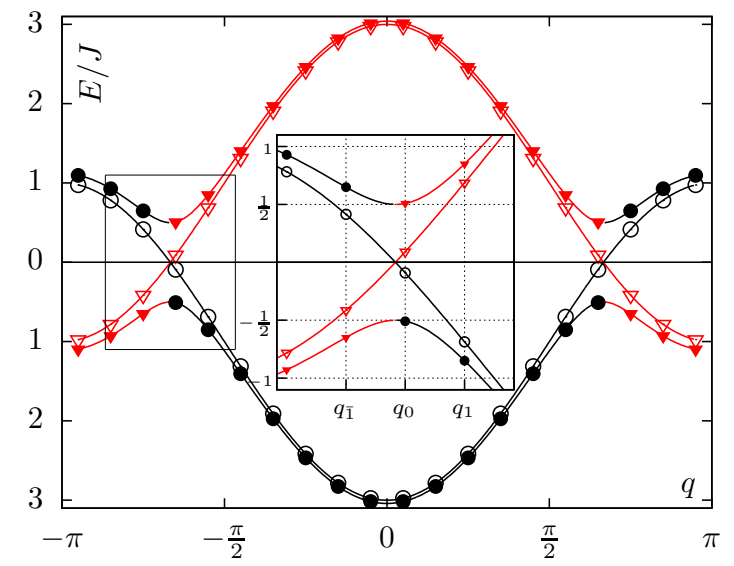

b)

Figura 5.1: Diagrama de bandas para rede de tamanho $L=20 \mathrm{com}$ aproximação de campo médio. Em a), os símbolos vazios (cheios) representam $\Lambda_{q} / J$ para momento $q$ com $\varepsilon_{0}=0\left(\varepsilon_{0}=-J\right)$. As linhas contínuas são apenas guia para os olhos. Os círculos (triângulos) são as quasipartículas de spin $\sigma=\uparrow(\downarrow)$. O acoplamento entre as cadeias é nulo, $\lambda=0$, e as bandas de energia para ambos os tipos de spin são contínuas. O potencial na aproximação de campo médio produz deslocamento $\varepsilon_{0}\left(-\varepsilon_{0}\right)$ para todos os níveis de energia para as quasipartículas de spin $\sigma=\uparrow(\downarrow)$, compatível com a ação de um campo magnético no efeito Zeeman. Em b), $\varepsilon=-J$ e os círculos (triângulos) cheios (vazios) representam as quasipartículas de spin $\sigma=\uparrow(\downarrow) \operatorname{com} \lambda=J / 2(\lambda=0)$. Verifica-se numericamente que o acoplamento entre as cadeias introduz o gap de energia $\Delta E=2|\lambda|$, criando bandas de condução e valência para cada tipo de spin. Devido a $\varepsilon_{0}$, há polarização de spin $\sigma$ para o estado fundamental. Figura reproduzida da Ref.[66] sob permissão acadêmica.

pela presença de dois poços ou mínimos de energia e um gap de energia $\Delta E_{g}=2|\lambda|$, em torno do ponto $X$, para cada spin $\sigma$ conforme ilustra o diagrama da Figura 5.1.

Dessa forma, observa-se duas bandas de valência e condução, uma para cada 
valor de spin, o que impede o comportamento crítico do modelo. Mais ainda, como $\varepsilon_{0}$ desloca os níveis de acordo com o spin, a composição do estado fundamental possui $\sum\left\langle Q_{q}\right\rangle \neq 0$, ou seja, há polarização em relação à variável de spin.

\subsection{Conclusão}

O comportamento de duas cadeias de spin interagentes foi investigado utilizando quasipartículas fermiônicas $\hat{\psi}_{k \sigma}$. A motivação para a introdução das quasipartículas foi remover efeitos de superfície, que são perturbações relevantes no estudo de nanoestruturas magnéticas. Em particular, essa abordagem explicou a emergência da polarização das quasipartículas no sistema bem como as regras de transição valência-condução, na aproximação de campo médio. 


\section{6}

\section{Conclusão e perspectivas}

Uma adaga afiada não é nada sem uma visão aguçada.

- Provérbio Klingon

As propriedades e características coletivas de sistemas complexos, sejam elas físicas ou matemáticas, emergem devido às interações entre as partes que os compõem. Nesta tese, utilizamos a teoria do momento angular como plataforma de investigação dessas propriedades e características, de certo modo contornando as barreiras que separam as áreas do conhecimento envolvidas. Em parte, isso foi consequência natural do emprego de operadores localizados de spin como elementos descritores dos sistemas, em conjunção com o emprego da teoria de grafos e redes. A adoção da teoria do momento angular permitiu a aproximação de conceitos e técnicas de áreas geralmente independentes. Por meio desse procedimento, verificamos que a modelagem de sistemas complexos via operadores de spin localizados permite a exploração de simetrias operatoriais, abordagem sobre a natureza fermiônica ou bosônica de operadores coletivos, além do desenvolvimento de novos métodos quantitativos.

Apesar de ter sido o tópico mais recente a ser estudado, a modelagem de sistemas epidemiológicos apresenta-se como o ápice da interdisciplinaridade desta tese. Nela, utilizamos operadores de spin localizados, estruturados e organizados por meio de grafos, para extrair a matriz de transição correspondente ao processo Markoviano do modelo SIS. Abordamos desde questões simples como a organização do espaço vetorial correspondente, passando pela reobtenção de modelos já bem estabelecidos, culminando no desenvolvimento de uma nova equação para processos 
Markovianos.

Parte da motivação desse estudo se deve à indagação a respeito do papel das flutuações estocásticas nos modelos epidêmicos, outrora negligenciadas por modelos compartimentais. Após um primeiro contato com o assunto ${ }^{1}$ a intuição indicava que uma dinâmica similar àquela do modelo de Ising fundamentava o processo, isto é, a existência de uma competição coletiva ordem-desordem. A dedução da matriz de transição exata a partir de primeiros princípios, com ajuda dos operadores de spin localizados, foi o primeiro passo nessa direção.

De fato, a conexão apropriada com o modelo de Ising se deu apenas no final de 2016, após o entendimento sobre a descrição do processo como um problema de Mecânica Estatística clássica por meio da definição de reservatório térmico (Apêndice 3.3). Essa conexão ainda requer testes mais sistemáticos e rigorosos a fim de determinar com precisão seu regime de validade - ou ao menos passar por testes que objetivam sua rejeição -, tarefas inviáveis no tempo alocado em nosso estudo corrente. Se tal hipótese for correta, os processos de disseminação de doenças podem usufruir do vasto arcabouço ferramental oriundo da Termodinâmica e Mecânica Estatística de fenômenos coletivos e transição críticas de fase associados ao modelo de Ising. Dentro do contexto social, tanto brasileiro como de outros países tropicais, podemos falar na questão da malária, uma das responsáveis pela grande mortalidade causada por doenças comunicáveis. Essa linha de pesquisa, entretanto, requer cooperação e coordenação com outra áreas do conhecimento, bem como financiamento adequado e compatível com as exigências socioeconômicas. Dentre nossas prioridades acadêmicas, temos boas expectativas quanto à essa linha, dado a evolução de novas técnicas computacionais e de novas gerações e tipos de processadores.

Quanto ao desenvolvimento da Eq. (3.65), temos grandes expectativas quanto à sua aplicação em outros problemas estocásticos. No caso dos modelos epidêmicos, há certa intuição a respeito de sua aplicação em conjunção com a aproximação de van Mieghem. O conjunto de informações até o momento sugere uma metodologia muito similares àquela da DFT. Essa intuição, fortemente baseada em experiências com problemas de estado sólido, será verificada com maiores detalhes em algum

\footnotetext{
${ }^{1}$ De passagem, convém mencionar que a primeira discussão sobre modelos epidêmicos ocorreu em meados de 2014 com o físico médico Enock Neto, então aluno de iniciação científica. Seu admirável e empolgante interesse sobre o assunto foi, sem dúvida nenhuma, meu maior incentivo para adentrar essa linha de pesquisa no final do ano seguinte. Com muita gratidão ofereço meus sinceros agradecimentos pelas discussões sobre toda a problemática, definições e meandros envolvidos.
} 
momento oportuno. Excluindo-se aproximações diretas acerca da distribuição de probabilidade, que são imposições muito fortes sobre as propriedades do modelo, a Eq. (3.65) também abre portas para o aplicação de aproximações ou técnicas perturbativas para o conteúdo espectral do operador simetrizado $\hat{\mathcal{H}}$. Este caso depende muito mais dos acoplamentos das interações do problema e da estrutura de vizinhança do grafo ou rede. Em particular, essa abordagem une de maneira serena estudos epidemiológicos com as metodologias de muitos corpos. Apesar da extensa discussão sobre o tópico ao longo do Capítulo 3, e também presentes nos Apêndices, exploramos apenas superficialmente as diversas possibilidades, seja por familiaridade prévia ou vantagem numérica. Por exemplo, a formulação de um esquema perturbativo empregando estados coerente, emprestando conceitos diretamente da Óptica Quântica. Todavia, nossa pretensão imediata é a formalização conceitual da entropia de Rényi para processos Markovianos. Sabemos que seu emprego em simulações de Monte Carlo quântico permite a descrição de problemas antes considerados intratáveis [172]. Isso por si só sugere que a entropia pode e deve ser utilizada também como elemento de redução de complexidade para descrever processos epidêmicos.

Já na parte mais física deste estudo, revisamos um problema bem conhecido de modelos de cavidade, sempre observado a teoria e propriedades oriundas do momento angular. A discussão sobre a natureza fermiônica e bosônica dos operadores de spin localizados culmina com indagação sobre a interpretação dos operadores anti-girantes como operadores dissipativos. Essa interpretação deriva da interação da radiação com átomos isolados, em que os conceitos de partículas são precisos. Assim como ocorre em matéria condensada, essa interpretação exige correções adicionais de acordo com o incremento da correlação atômica. Aqui, nos limitamos às correções de efeito finito devido ao número de fótons na cavidade ideal. Nesse regime e em conjunção com a simetria de gauge de primeiro tipo, descrevemos o sistema como um problema de interação spin-órbita. Ao tratar o problema dessa maneira evitamos a fermionização ou bosonificação dos campos de matéria, mais adequado para sistemas finitos. Em contrapartida, com a Eq. (4.9) mostramos que muito embora os operadores anti-girantes possuam contribuição nula para a energia, seus efeitos são relevantes para a distribuição de probabilidade de estados do sistema. A partir dessa premissa e para o caso de cavidade ideal não-dissipativa, concluímos que 
a presença de operadores anti-girantes produz interferência entre espécies atômicas que não interagem diretamente.

Por fim, nossos resultados também incluem o comportamento e propriedades espectrais para duas cadeias de spin interagentes. Esse modelo, muito mais especializado que os demais assuntos, é matéria constante e situa-se na interface de Mecânica Estatística, matéria condensada e sistemas exatamente integráveis. Apesar da conexão com o momento angular ser bastante evidente nestes problemas, seu emprego raramente se transforma em propriedades úteis devido ao elevado número de graus de liberdade. Contudo, essa situação se reverte no regime de nanometros, área que observou intenso desenvolvimento nas duas últimas décadas, devido ao grande número potencial tecnológico. No nosso caso, o estudo de duas cadeia paralelas é bastante simplificado, posto que definimos o problema diretamente com férmions. A partir da subsequente investigação das relações algébricas, introduzimos quasipartículas fermiônicas por meio das transformações de Bogolibov, dotadas de um grau de liberdade compatível com o spin (pseudo-spin). Em vistas de nossos resultados, concluímos que o acoplamento entre as diferentes cadeias gera um gap de energia. Assim, observa-se a emergência de bandas de condução e valência das cadeias, bem como as suas respectivas polarizações. Questões quanto ao transporte de spin e geração de ondas de spin por efeito Seeback ou interação com fônons ainda são questão em aberto. Esperamos abordá-las futuramente, com maior foco sobre materiais reais. 


\section{Apêndice}

\section{Grafos e redes}

A descrição formal de um grafo $G$ descreve uma estrutura matemática formado por um conjunto de vértices $V=\left\{v_{0}, v_{1}, \ldots\right\}$ e por um conjunto de arestas $E=\left\{e_{0}, e_{1} \ldots\right\}$. A ideia central que circunda esse conceito é descrever relações dois-a-dois entre vértices distintos, por meio das arestas, as quais representam conexões ou correlações. Essa abordagem é muito ampla e permite a descrição do inter-relacionamento de objetos reais, sejam eles pessoas, empresas, moléculas, etc. Dentre as suas inúmeras aplicações, grafos encontram um ambiente adequado para descrever processos epidêmicos na modelagem baseada em agentes [13].

A Teoria de Grafos é um ramo muito rico e bastante desenvolvido da Matemática, que implica numa extensa carga conceitual, com diversas propriedades relevantes [173]. Nesta tese, utilizamos o conceito de grafos e redes, como uma estrutura subjacente, onde ocorre algum tipo de dinâmica. No caso de modelos epidêmicos, a dinâmica é o processo de disseminação de doenças. Assim, apenas algumas propriedades são de nosso interesse imediato. Portanto, não convém escrever um tratado sobre o tema aqui, de modo que nos restringimos às propriedades relevantes.

A primeira propriedade, e extensivamente utilizada ao longo desta tese, findase na matriz de adjacência $A \equiv A(G)$, do grafo $G$. A matriz $A$ tem como papel fundamental atuar como o descritor primário de um grafo. Existem diversas maneiras de se representar uma matriz de adjacência. Aqui, escolhemos a mais tradicional e simples, que é uma representação matricial $N \times N$, onde $N$ é o número de vértices em $V$. Para manter consistência com o corpo principal deste estudo, convém representar a matriz na base $|i\rangle$, com $i=0,1, \ldots, N-1$, que representa cada vértice. 
Em termos dessa base, a matriz $A$ fica

$$
A=\sum_{i j=0}^{N-1} A_{i j}|i\rangle\langle j| .
$$

Os coeficientes $A_{i j}=1$ se o vértice $j$ possui uma conexão com o vértice $i$, ou anula-se caso contrário. Quando $A_{i j} \neq A_{j i}$, diz-se que o grafo é direcionado, e não-direcionado caso contrário.

Várias propriedades de grafos podem ser extraídas diretamente da matriz $A$. Por exemplo, o número de conexões que partem do $i$-ésimo vértice, mais conhecido como grau $\kappa_{i}$, é obtida por meio da soma da coluna de $A$ correspondente ao vértice $i$, isto é,

$$
\kappa_{i}=\sum_{j} A_{j i}=\sum_{j}\langle j|A| i\rangle \equiv\langle\phi|A| i\rangle .
$$

Na expressão acima, aproveitamos a oportunidade e também definimos o vetor $|\phi\rangle=$ $\sum_{j}|j\rangle$. Analogamente, pode-se calcular o grau de incidência do vértice $i$, ou seja, o número total de arestas que incidem no vértice $i$ :

$$
\kappa_{i}^{\prime}=\sum_{j} A_{i j}=\sum_{j}\langle i|A| j\rangle=\langle i|A| \phi\rangle .
$$

Se o grafo for não-direcionado, então $\kappa_{i}=\kappa_{i}^{\prime}$.

Seja a matriz $\rho=|\phi\rangle\langle\phi|$, cujos elementos de matriz são $\rho_{i j}=1$. O grau médio para os $N$ vértices do grafo $G$ é dado pela seguinte expressão:

$$
\left\langle\kappa_{G}\right\rangle=\frac{1}{N} \sum_{i=0}^{N-1} \kappa_{i}=\frac{\operatorname{Tr}(\rho A)}{\operatorname{Tr}(\rho)}=\frac{1}{N} \operatorname{Tr}(\rho A) .
$$

A propriedade cíclica do traço também permite concluir que o grau incidente médio $\left\langle\kappa_{G}^{\prime}\right\rangle=\left\langle\kappa_{G}\right\rangle$, como esperado. Já o valor médio do produto dos graus incidente e emergente produz a expressão

$$
\left\langle\kappa_{G}^{\prime} \kappa_{G}\right\rangle=\frac{\sum_{i}\langle i|A| \phi\rangle\langle\phi|A| i\rangle}{\operatorname{Tr}(\rho)}=\frac{1}{N} \operatorname{Tr}\left(\rho A^{2}\right) .
$$

Para grafos não-direcionados, $\left\langle\kappa_{G}^{\prime} \kappa_{G}\right\rangle=\left\langle\kappa_{G}^{2}\right\rangle$, o que nos permite definir a respectiva variância $\sigma_{G}^{2}=\left\langle\kappa_{G}^{2}\right\rangle-\left\langle\kappa_{G}\right\rangle^{2}$. Além disso, a partir da identidade $\rho^{2}=N \rho$ e definido-se a matriz

$$
A^{\prime}=\rho A=\sum_{i j} \kappa_{j}|i\rangle\langle j|,
$$


cujos autovalores são $a_{i}^{\prime}(i=0,1, \ldots, N-1)$, temos

$$
\begin{aligned}
\left\langle\kappa_{G}\right\rangle & =\frac{\sum_{i} a_{i}^{\prime}}{N}, \\
\left\langle\kappa_{G}^{2}\right\rangle & =\frac{1}{N^{2}} \operatorname{Tr}\left[\left(A^{\prime}\right)^{T} A^{\prime}\right]=\frac{\sum_{i} b_{i}^{\prime}}{N^{2}}, \\
\sigma_{G}^{2} & =\frac{1}{N^{2}} \sum_{i j}\left[b_{i}^{\prime} \delta_{i j}-a_{i}^{\prime} a_{j}^{\prime}\right],
\end{aligned}
$$

onde $b_{i}^{\prime}$ são os autovalores correspondentes a $\left(A^{\prime}\right)^{T} A^{\prime}$.

Uma propriedade muito útil é facilmente deduzida diretamente da Eq. (1.6):

$$
\left(A^{\prime}\right)^{2}=N\left\langle\kappa_{G}\right\rangle A^{\prime}
$$

Se aplicada à equação de autovalor e autovetor, conclui-se que $a_{i}^{\prime}=0$ ou $a_{i}^{\prime}=N\left\langle\kappa_{G}\right\rangle$. Note que todos os autovalores são maiores ou iguais a zero e, portanto, pelo teorema de Perron-Frobenius autovetor correspondente ao maior autovalor é não degenerado. Logo, apenas um autovalor vale $a_{0}^{\prime}=N\left\langle\kappa_{G}\right\rangle$. Argumento semelhante é utilizado para mostrar que apenas um autovalor $b_{0}^{\prime}=N^{2}\left\langle\kappa_{G}^{2}\right\rangle$ da matriz simétrica $\left(A^{\prime}\right)^{T} A^{\prime}$ é não nulo. Porém, por ser uma matriz simétrica $b_{0}^{\prime} \geqslant\left(a_{0}^{\prime}\right)^{2}$, de tal maneira que

$$
\sigma_{G}^{2}=\frac{b_{0}^{\prime}-\left(a_{0}^{\prime}\right)^{2}}{N^{2}} \geqslant 0
$$

como esperado. Por se tratarem dos maiores autovalores, o método da potência é muito bem vindo.

\section{$1.1 \quad$ Redes}

Outra ferramenta utilizada para modelar relações dois-a-dois é a teoria de rede, muitas vezes empregada como sinônimo de grafos. Mas o que é exatamente uma rede? Quais são suas classes e propriedades? De acordo com Newman [99], uma rede é um grafo. Já Albert e Barábasi [55], argumentam que redes são estruturas caracterizadas por suas propriedades topológicas como grau médio, conectividade,

etc. Essas definições não são equivalentes entre si, já que existem diferentes grafos com mesmos valores topológicos.

O cálculo das estatísticas do grafo, como grau médio e variância entre outras, é realizado a partir de potências da matriz $A(G)$, correspondente ao grafo $G$. Ao mesmo tempo, a menção da palavra estatística nos remete ao conceito de acaso, que 
grosso modo expressa a variabilidade de conexões entre vértices. Portanto, é razoável considerar que as arestas são geradas de acordo com uma distribuição de probabilidade $p(E)$. Assim como qualquer distribuição de probabilidade, $p(E)$ fundamenta-se sobre seu espaço amostral, constituído por um ensemble de grafos, $\mathcal{N}$. Dessa maneira, o grafo $G_{k}$ nada mais é do que uma amostra de $\mathcal{N}=\left\{G_{0}, G_{1}, \ldots, G_{k}, \ldots\right\}$.

A existência de $p(E)$ não altera a análise individual já realizada para os grafos. Porém, semeia a ideia que todos os grafos de $\mathcal{N}$ tem algo em comum. Esse conceito é intuitivo e sempre foi explorado no estudo de teoria de redes aleatórias. Por exemplo, numa rede aleatória com distribuição uniforme de probabilidade para as arestas, $p$, o número total de arestas médio vale $p N(N-1) / 2$. Isso não quer dizer que todos os grafos aleatórios, com probabilidade $p$, tenham esse número de arestas. Alguns podem ter poucas arestas, por exemplo.

A partir do conceito de ensemble de grafos, podemos definir o estimador para a matriz de adjacência,

$$
\langle A\rangle=\frac{1}{m} \sum_{k=0}^{m-1} A\left(G_{k}\right),
$$

com $G_{k} \in \mathcal{N}$ e $m \gg 1$. Para o exemplo anterior do grafo aleatório, temos o seguinte elemento de matriz $(\langle A\rangle)_{i j}=p\left(1-\delta_{i j}\right)$. A matriz correspondente possui $p N(N-1)$ conexões totais e, portanto, $p N(N-1) / 2$ arestas já que o grafo não é direcionado. Além do primeiro momento, define-se os momento de ordem $\ell$ :

$$
\left\langle A^{\ell}\right\rangle=\frac{1}{m} \sum_{k=0}^{m-1} A^{\ell}\left(G_{k}\right)
$$

$\operatorname{com} \ell=0,1,2, \ldots$ inteiro.

Para conectar as propriedades do ensemble com a definição de rede de Albert e Barabási, basta impor condições restritivas aos grafos que compõem $\mathcal{N}$, em analogia ao ensemble microcanônico [53]. Como exemplo inicial, suponha que o grafo $G_{j}$ do ensemble é tal que o grau médio $\left\langle\kappa_{j}\right\rangle$ situa-se entre $\kappa$ e $\kappa+\delta \kappa$. Aqui, é suficiente especificar que $\delta \kappa$ decresce com o incremento de $N$. Sob essas circunstâncias, o estimador na Eq. (1.10) torna-se

$$
\langle A\rangle=\frac{1}{m} \sum_{j=0}^{m-1} A\left(G_{j}\right)\left[\theta\left(\left\langle\kappa_{j}\right\rangle-\kappa\right) \theta\left(\kappa+\delta \kappa-\left\langle\kappa_{j}\right\rangle\right)\right],
$$

onde $\theta(x)$ é a função degrau. Em relação ao parâmetro $\kappa$, todos os grafos do ensemble são equivalentes, e portanto, cada grafo é também um bom representativo da rede, 
compatibilizando as duas definições de redes.

Despeito a discussão em torno de redes como um ensemble de grafos, na prática, utiliza-se apenas um único grafo, geralmente com $N \gg 1$. A justificativa para isso baseia-se em ideias similares àquelas da Hipótese Ergódica [53]. Ao invés de utilizar um ensemble de grafos, particiona-se um grafo $G$ em $M \ll N$ subgrafos $g_{k}(k=0,1, \ldots, M-1)$, com $n \approx N / M$ vértices cada um. De certa maneira, o conjunto de subgrafos $\left\{g_{0}, g_{1}, \ldots, g_{M-1}\right\}$ forma um ensemble.

Considere o grafo $G$ da Figura 1.1. $G$ é particionado em $M=4$ grafos menores $\left\{g_{0}, \ldots, g_{M-1}\right\}$. Durante a partição, as arestas entre subgrafos diferentes são desprezadas de modo a garantir o isolamento entre os vértices de cada subgrafo. Então, podemos analisar cada grafo $g_{k}$ individualmente, incluindo aí, o cálculo de suas estatísticas, que representamos por $\left\langle s_{k}\right\rangle$. Para $N \gg M>1$, define-se o estimador da estatística $s$ para o grafo $G$ :

$$
\langle s\rangle_{G}=\frac{1}{M} \sum_{k=0}^{M-1}\left\langle s_{k}\right\rangle
$$

Essa estimativa melhora quanto menor for o número de ligações quebradas para se construir os $M$ subgrafos e, por este motivo, a relevância da condição $N \gg 1 .^{2}$

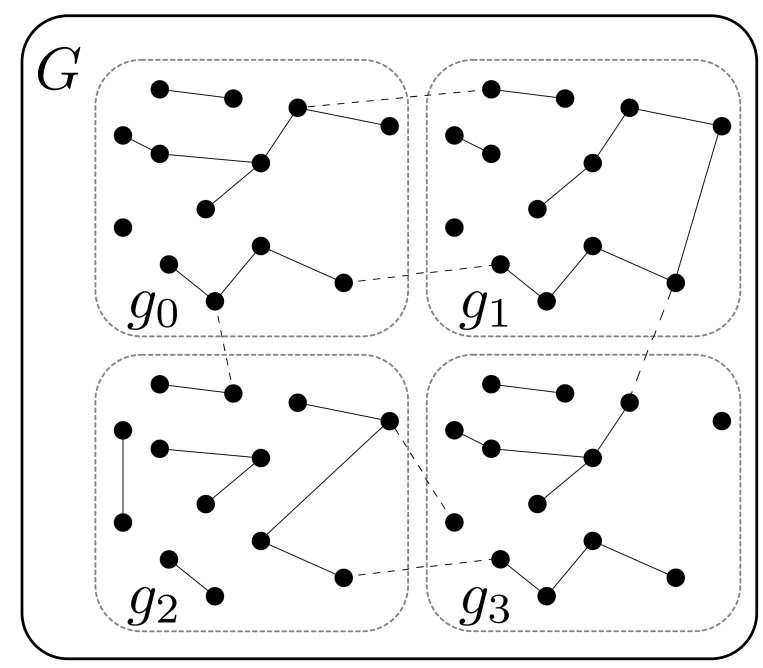

Figura 1.1: Grafo $G$ com $N=52$ vértices. O grafo é particionado em 4 grafos menores $g_{0}, g_{1}, g_{2}$ e $g_{3}$, cada um com $n=13$ vértices. As arestas pontilhadas não fazem parte dos grafos $g_{k}$, mas sim do grafo $G$. As propriedades topológicas de cada grafo $g_{k}$ representam muito bem as propriedades topológicas médias do grafo $G$.

\footnotetext{
${ }^{2}$ Nem todo grafo apresenta a propriedade de auto-mediação, isto é, partes isoladas tornam-se bons representativos das propriedades do todo, embora sujeitos a desvios estatísticos.
} 


\subsection{Combinação linear de Grafos}

Considere um sistema é constituído por $N$ componentes interagentes, que são identificadas por vértices. No entanto, existem dois conjuntos de regras para descrever as interações entre os vértices, digamos, tipo $\mathcal{A}$ e $\mathcal{B}$. De acordo, constróise dois grafos não-direcionais $G_{A}$ e $G_{B}$, respectivamente, um para cada regra de interação. Cada grafo possui uma matriz de adjacência correspondente, $A$ e $B$.

Um cenário muito frequente decorre da tentativa de construir um novo grafo, $G_{C}$, que represente a combinação do conteúdo de ambos grafos $G_{A}$ e $G_{B}$. Essa situação é bastante diferente daquelas descritas anteriormente, pois tenta interpolar diferentes regras ou ensembles, no caso de redes. Uma possibilidade consiste na combinação linear

$$
C=\alpha A+\beta B
$$

onde $\alpha$ e $\beta$ são coeficientes reais a ser determinados. Apesar de serem indeterminados, no momento, eles não podem ser arbitrários. A razão disso é que, seja lá qual forem seus valores, a variância $\sigma_{C}^{2}$ de $G_{C}$ dever maior ou igual a zero, assim como o grau médio $\left\langle\kappa_{C}\right\rangle$.

Vejamos então como se comportam estas estatísticas. O grau médio de $G_{C}$ é dado pela expressão

$$
\left\langle\kappa_{C}\right\rangle=\frac{1}{N}\left[\alpha \sum_{i k} A_{i k}+\beta \sum_{i k} B_{i k}\right]=\alpha\left\langle\kappa_{A}\right\rangle+\beta\left\langle\kappa_{B}\right\rangle,
$$

em que tanto $\left\langle\kappa_{A, B}\right\rangle \geqslant 0$. Em contrapartida, a variância é expressa da seguinte maneira:

$$
\begin{aligned}
\sigma_{C}^{2} & =\left\langle\kappa_{C}^{2}\right\rangle-\left\langle\kappa_{C}\right\rangle^{2} \\
& =\alpha^{2} \sigma_{A}^{2}+\beta^{2} \sigma_{B}^{2}+\frac{\alpha \beta}{N}\left[\operatorname{Tr}(\rho A B)+\operatorname{Tr}(\rho B A)-2 N\left\langle\kappa_{A}\right\rangle\left\langle\kappa_{B}\right\rangle\right], \\
& \equiv \alpha^{2} \sigma_{A}^{2}+\beta^{2} \sigma_{B}^{2}+2 \alpha \beta \sigma_{A B} \geqslant 0 .
\end{aligned}
$$

As parcelas $\alpha^{2} \sigma_{A}^{2}$ e $\beta^{2} \sigma_{B}^{2}$ são necessariamente positivas. Por outro lado, a partir da desigualdade $\sum_{i} \sum_{l m} \sum_{j} A_{j m} B_{m l} \leqslant \sum_{i j} A_{i j} \sum_{l m} B_{l m}$, temos

$$
\operatorname{Tr}(\rho A B+\rho B A)=\sum_{i m}\left(\sum_{l} A_{l m}\right) B_{m i}+\sum_{i m}\left(\sum_{l} B_{l m}\right) A_{m i} \leqslant 2 N\left\langle\kappa_{A}\right\rangle\left\langle\kappa_{B}\right\rangle .
$$

Ora, segue que a parcela referente à covariância $\sigma_{A B}=(1 / 2)[\operatorname{Tr}(\rho A B+\rho B A)-$ $\left.2 N\left\langle\kappa_{A}\right\rangle\left\langle\kappa_{B}\right\rangle\right]$ da Eq. (1.16) pode vir a adotar valores negativos. 
Levando em conta que $\alpha$ e $\beta$ não são independentes, pois na realidade imitam pesos, podemos impor a relação $\beta=1-\alpha$. Essa imposição ${ }^{3}$ permite determinar o valor de $\alpha=\alpha^{\prime}$ como o parâmetro que minimiza a variância $\sigma_{C}^{2}$ :

$$
\alpha^{\prime}=\frac{\sigma_{B}^{2}-\sigma_{A B}}{\sigma_{A}^{2}+\sigma_{B}^{2}-2 \sigma_{A B}} .
$$

A fim de ilustrar nosso argumento, considere as matrizes de adjacência $A$ de um grafo aleatório com $p=3 / 4 ; B$ onde apenas um vértice se conecta com todos os outros vértices; e $C=\alpha A+\beta B$ :

$$
\begin{aligned}
A & =\left[\begin{array}{lllll}
0 & 1 & 1 & 1 & 0 \\
1 & 0 & 1 & 1 & 1 \\
1 & 1 & 0 & 1 & 0 \\
1 & 1 & 1 & 0 & 1 \\
0 & 1 & 0 & 1 & 0
\end{array}\right], \quad B=\left[\begin{array}{lllll}
0 & 1 & 1 & 1 & 1 \\
1 & 0 & 0 & 0 & 0 \\
1 & 0 & 0 & 0 & 0 \\
1 & 0 & 0 & 0 & 0 \\
1 & 0 & 0 & 0 & 0
\end{array}\right] \\
C & =\left[\begin{array}{ccccc}
0 & \alpha+\beta & \alpha+\beta & \alpha+\beta & \beta \\
\alpha+\beta & 0 & \alpha & \alpha & \alpha \\
\alpha+\beta & \alpha & 0 & \alpha & 0 \\
\alpha+\beta & \alpha & \alpha & 0 & \alpha \\
\beta & \alpha & 0 & \alpha & 0
\end{array}\right],
\end{aligned}
$$

que produzem $\sigma_{C}^{2}=(1 / 25)\left[14 \alpha^{2}-6 \alpha(1-\alpha)+36(1-\alpha)^{2}\right]$ (ver Figura 1.2).

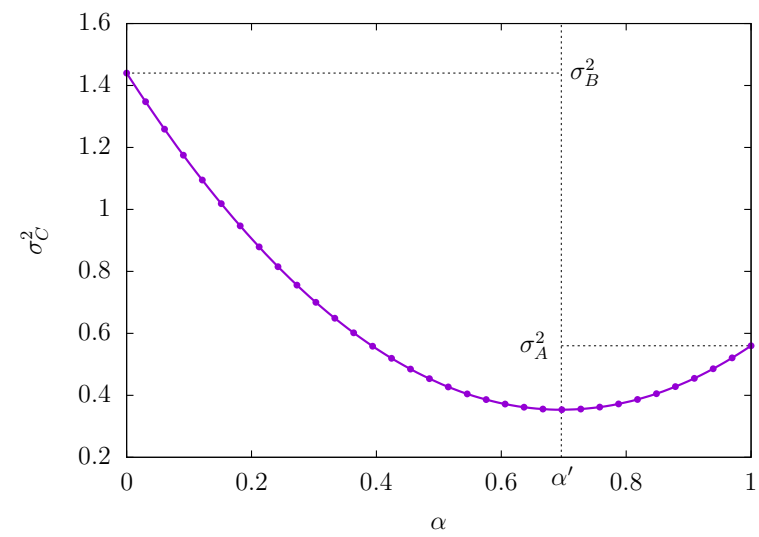

Figura 1.2: Variância do grafo $G_{C}$ formado a partir da combinação dos grafos $G_{A}$ e $G_{B}$ para $N=5$ vértices. Os grafos $G_{A}$ e $G_{B}$ descrevem relacionamentos distintos entre os vértices. A combinação $C=\alpha A+\beta B$ produz menor variância quando $\alpha=\alpha^{\prime}$.

\footnotetext{
${ }^{3}$ Em geral, minimiza-se o valor de $\sigma_{C}^{2}$ mantendo-se os vínculos $0 \leqslant x \leqslant 1$ e $\left\langle\kappa_{C}\right\rangle \geqslant 0$.
} 


\section{Apêndice}

\section{Simetrias finitas e implementação computacional}

Um dos grandes benefícios de simetrias é a redução do esforço computacional necessário para se calcular elementos de matriz. Sob considerações de simetria, matrizes são quebradas em um conjunto de blocos disjuntos, cada um indexado por um número - o número quântico para Mecânica Quântica, por exemplo. Os cálculos numéricos de matriz de transição $\hat{T}$ e do gerador de translações temporais $\hat{H}$ não são exceções.

As interações nos modelos epidêmicos estão intrinsecamente relacionadas com as propriedades da matriz de adjacência. Estas por sua vez podem possuir simetrias geométricas, como invariância por translação, rotação, etc. De acordo com o teorema de Cayley [43], transformações geométricas finitas são isomórficas ao grupo de permutação. Portanto, este grupo é utilizado para gerar um espaço vetorial invariante por permutações cíclicas. Se a matriz de adjacência $A$ de um grafo possuir tal simetria, o uso das bases correspondentes reduzem $\hat{T}$ em $N$ blocos disjuntos. Se simetrias adicionais forem consideradas, como por exemplo, conservação do número de infectados, cada um dos $N$ blocos subdivide-se em blocos menores, como acontece no caso de grafos completos.

\subsection{Espaço vetorial}

A base ingênua descreve o estado de saúde de cada um dos $N$ agentes, totalizando $2^{N}$ vetores distintos. Esses elementos são os vetores configuração $\left|C_{\mu}\right\rangle \equiv|\mu\rangle$ descritos na Eq. (3.13) e exemplificado na Figura 2.3. A representação binária é con- 
veniente caso se faça uso de operações lógicas para mimicar transformações, ou para se manter um código numérico independente de linguagem computacional. Outra alternativa viável, caso exista suporte eficiente da linguagem, é a representação da configuração por meio de strings. ${ }^{4}$

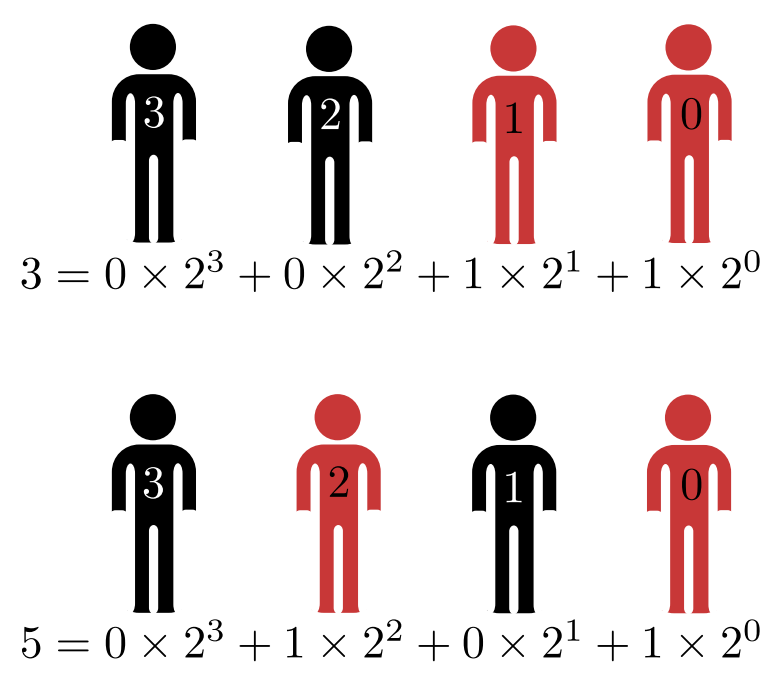

Figura 2.3: Representação binária $\mu=3$ e 5 para $N=4$.

Seja $\hat{P}$ o operador de permutação cíclica. Sua ação sobre uma configuração $|\mu\rangle$ translada cada configuração $\sigma_{k}$ para o vértice $k+1$. A exceção é o vértice $N-1$, cuja variável $\sigma_{N-1}$ vai para a posição correspondente ao vértice $k=0$. Por exemplo,

$$
\hat{P}|0101\rangle=|1010\rangle
$$

Naturalmente, a ação sucessiva de $N$ permutações retorna o estado ao seu estado inicial. Logo $\hat{P}^{N} \equiv \mathbb{1}$. Portanto, os autovalores de $\hat{P}$ são $\mathrm{e}^{-2 \imath \pi p / N}$, onde $p=$ $0,1,2, \ldots, N-1 .^{5}$ Um vetor $\left|\mu_{p}\right\rangle$ é invariante por permutações cíclicas se cumprir a equação de autovalor

$$
\hat{P}\left|\mu_{p}\right\rangle=\mathrm{e}^{-2 \imath \pi p / N}\left|\mu_{p}\right\rangle
$$

Ora, dado o vínculo $\hat{P}^{N} \equiv \mathbb{1}$, uma escolha adequada consiste em escolher uma configuração básica $|\mu\rangle$ e, a partir desta, gerar todas as suas $N-1$ permutações.

\footnotetext{
${ }^{4}$ Os trechos de código computacional exibidos aqui são escritos em Python, uma vez que a linguagem Python (versão 3.5) tem se mostrado mais popular que o bom e velho Fortran. Contudo, recomenda-se o uso de Fortran ou C, especialmente se paralelização por meio de openMP ou MPI for desejada. Destaca-se que a linguagem Python (versão 3.5) possui algumas diferenças em relação à versão 2.7, especialmente quanto ao papel dos geradores xrange (2.7) e range (3.5).

${ }^{5}$ Esse é o argumento por trás da seleção uniforme dos comprimentos de onda da transformada de Fourier. Se porventura houvesse ganho de fase após as $N$ permutações, a distribuição não necessariamente seria uniforme. De fato, essa é a ideia por trás do ansatz de Bethe [74].
} 
Tabela 2.1: Autovetores da base cíclica para $N=4$. A primeira coluna exibe o número de infectados.

\begin{tabular}{lllll}
\hline \hline$n$ & $p=0$ & $p=1$ & $p=2$ & $p=3$ \\
0 & $\left|0_{0}\right\rangle$ & & & \\
1 & $\left|1_{0}\right\rangle$ & $\left|1_{1}\right\rangle$ & $\left|1_{2}\right\rangle$ & $\left|1_{3}\right\rangle$ \\
2 & $\left|3_{0}\right\rangle$ & $\left|3_{1}\right\rangle$ & $\left|3_{2}\right\rangle$ & $\left|3_{3}\right\rangle$ \\
2 & $\left|5_{0}\right\rangle$ & & $\left|5_{2}\right\rangle$ & \\
3 & $\left|7_{0}\right\rangle$ & $\left|7_{1}\right\rangle$ & $\left|7_{2}\right\rangle$ & $\left|7_{3}\right\rangle$ \\
4 & $\left|15_{0}\right\rangle$ & & & \\
\hline \hline
\end{tabular}

Desse modo, todos os vetores relacionados por permutações ficam atrelados uns aos outros. Assim,

$$
\left|\mu_{p}\right\rangle=\frac{1}{\mathcal{N}_{p \mu}} \sum_{k}\left(\mathrm{e}^{2 \imath \pi p / N} \hat{P}\right)^{k}|\mu\rangle .
$$

O número $\mathcal{N}_{p \mu}$ serve para normalizar o vetor. É fácil ver que $\left|\mu_{p}\right\rangle$ satisfaz a equação $\hat{P}\left|\mu_{p}\right\rangle=\mathrm{e}^{-2 \imath \pi p / N}\left|\mu_{p}\right\rangle$. Como exemplo, considere o vetor

$$
\left|1_{0}\right\rangle=\frac{|1000\rangle+|0100\rangle+|0010\rangle+|0001\rangle}{2} .
$$

Para se determinar todos os vetores da base cíclica, precisa-se resolver quatro problemas. O primeiro problema é a determinação do vetor básico (representativo) sobre o qual se atua o operador $\sum_{k}\left(\mathrm{e}^{2 \imath \pi p / N} \hat{P}\right)^{k}$. O critério mais simples para definir o representativo é selecionar a configuração com o menor valor $\mu$, dentre todas as configurações relacionadas apenas por permutações cíclicas. Por exemplo, as configurações $|1\rangle,|2\rangle,|4\rangle$ e $|8\rangle$ são relacionadas por permutações mas o vetor $|1\rangle$ possui o menor índice inteiro (ver Tabela 2.1).

O problema seguinte, e mais complicado, é a determinação da normalização $\mathcal{N}_{p \mu}$. Para entender essa afirmação, considere $\hat{P}\left|\mu_{p}\right\rangle+\left|\mu_{p}\right\rangle=\left(1+\mathrm{e}^{-2 \imath \pi p / N}\right)\left|\mu_{p}\right\rangle$. Para determinados valores de $p$, a soma das exponencias pode se cancelar, produzindo o vetor nulo, que não faz parte da base. Como exemplo, considere o vetor $\left|0_{p}\right\rangle=$ $\mathcal{N}_{p 0}^{-1}\left(1+\mathrm{e}^{-2 \imath \pi p / N}+\mathrm{e}^{-4 \imath \pi p / N}+\mathrm{e}^{-6 \imath \pi p / N}\right)|0000\rangle$, que se anula para $p \neq 0$.

De maneira geral, temos

$$
\left\langle\mu_{p} \mid \mu_{p}\right\rangle=\frac{N}{\mathcal{N}_{p \mu}}\left\langle\mu \mid \mu_{p}\right\rangle \equiv \frac{N R_{p \mu}}{\mathcal{N}_{p \mu}^{2}} .
$$

Observe que o produto escalar $\left\langle\mu \mid \mu_{p}\right\rangle=1$ se não existir repetição da configuração $|\mu\rangle$, que resulta em $\mathcal{N}_{p \mu}=\sqrt{N}$. Caso exista repetições, digamos a cada $\ell$ permutações, 
então

$$
R_{p \mu}=\sum_{m=0}^{N / \ell-1} \mathrm{e}^{2 \imath \pi m \ell p / N} .
$$

Quando $R_{p \mu}=0$, temos combinações proibidas, que permite a contabilidade correta da dimensão do espaço vetorial. Caso contrário, o vetor pertence à base e possui norma $\mathcal{N}_{\mu}=\sqrt{N R_{\mu}}$.

Com os detalhes fora do caminho, a construção da base é bastante simples. Primeiro, note que permutações cíclicas não mudam o número de agentes infectados. Isto quer dizer que o número total de infectados e a permutação comutam. O número total de infectados consiste no número de bits na representação binária do representativo $\mu$. Funções especializadas como bit_count, nativas ou da biblioteca gmpy2, são bem otimizadas. Exibimos uma implementação com intuito pedagógico no Algoritmo 1, utilizando apenas operações discretas. Se representação de string for utilizada, a função count produz resultados equivalentes.

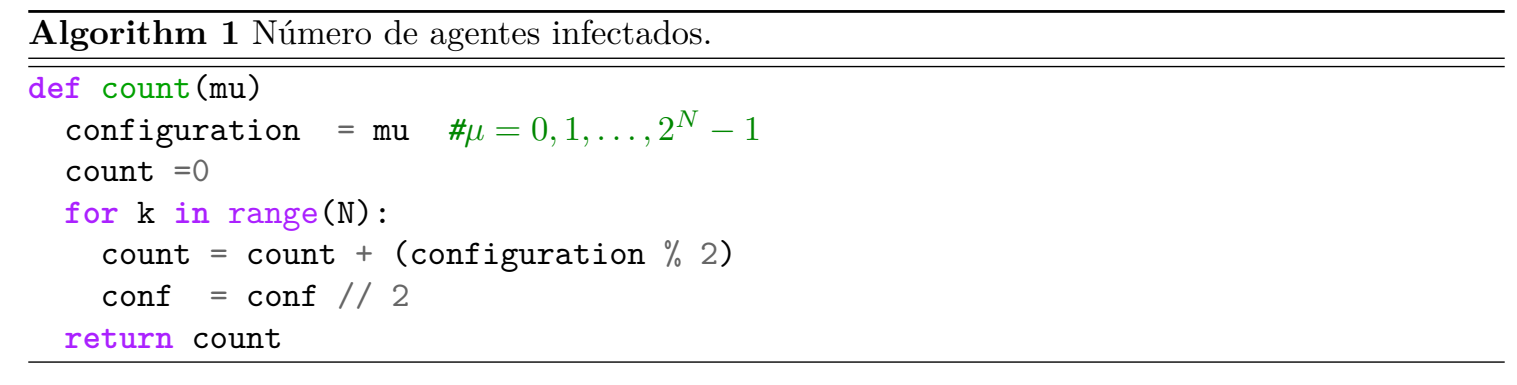

O passo seguinte consiste na implementação da operação de permutação cíclica. Aqui, existem diversas opções, dependendo da implementação da configuração. Para strings, o comando new_conf $=\operatorname{conf}[-1]+\operatorname{conf}[:-2]$ excuta a permutação cíclica. Para base binária, a função ISHFTC executa essa operação. Equivalentemente, operações de matemática discreta, como resto \% e divisão inteiro //, produzem o mesmo resultado $\mu^{\prime}=\left(2 \mu \% 2^{N}\right)+\left(2 \mu / / 2^{N}\right)$.

No Algoritmo 2, exibimos um código computacional para determinar o inteiro representativo $\mu$, bem como o número de repetições $\ell$ após $N$ permutações. Com essas informações, determina-se $R_{p \mu}$ e, portanto, a norma $\mathcal{N}_{p \mu}$.

As configurações representativas e as respectivas normas são então armazenadas em duas listas ou dicionários. O uso de dicionários ou mecanismos de busca por hash são recomendados para $N>20$, pois minimizam operações de busca. 


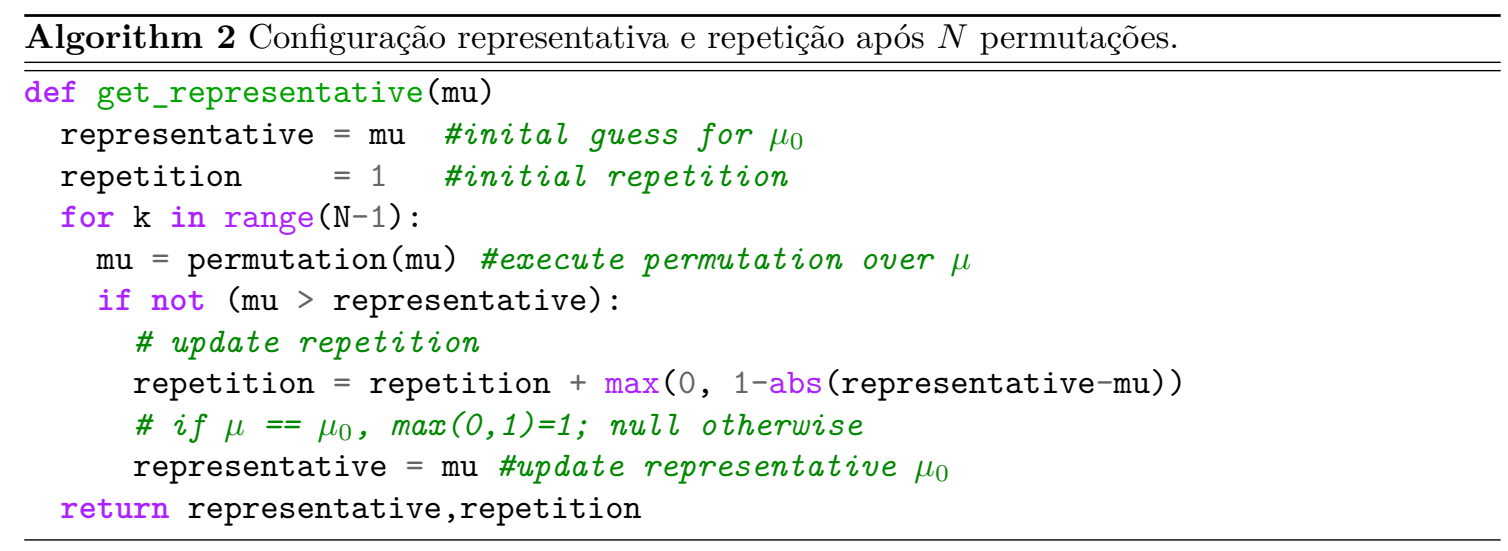

\subsection{Representação matricial}

O setor mais relevante para processos epidêmicos ocorre para $p=0$. No que segue, nos restringimos apenas a esse setor. Considere uma matriz de adjacência $A$ que seja invariante por permutações cíclicas. A matriz $A$ pode contemplar outras simetrias finitas. Neste caso, o operador $\hat{T}$ também goza de tal propriedade, que implica $[\hat{T}, \hat{P}]=0$. Logo,

$$
\hat{T}\left|\mu_{0}\right\rangle=\frac{1}{\mathcal{N}_{p \mu}} \sum_{k=0}^{N-1} \hat{P}^{k} \hat{T}|\mu\rangle .
$$

Ora, a vantagem nessa observação é imediata: ao invés de calcular a ação de $\hat{T}$ sobre cada configuração gerada por permutações, calcula-se apenas $\hat{T}|\mu\rangle$. As configurações geradas, no entanto, podem ser permutações de configurações representativas. Estas por sua vez diferem da configuração representativa por uma fase. Por exemplo, considere $\hat{T}\left|7_{0}\right\rangle$ para $N=3$ :

$$
\begin{aligned}
\hat{T}\left|7_{0}\right\rangle & =\frac{1}{\mathcal{N}_{7}} \sum_{k=0}^{2} \hat{P}^{k} \hat{T}|7\rangle=\frac{\gamma}{\mathcal{N}_{7}} \sum_{k=0}^{2} \hat{P}^{k}(|3\rangle+|5\rangle+|6\rangle) \\
& =\frac{\gamma}{\mathcal{N}_{7}} \sum_{k=0}^{2} \hat{P}^{k}\left(|3\rangle+\hat{P}|3\rangle+\hat{P}^{2}|3\rangle\right)=\left(3 \gamma \frac{\mathcal{N}_{3}}{\mathcal{N}_{7}}\right)\left|3_{0}\right\rangle \\
& =\sqrt{3} \gamma\left|3_{0}\right\rangle .
\end{aligned}
$$

A construção dos elementos de matriz de $\hat{T}$ requer o cálculo de contribuições provenientes de dois tipos de fontes: de um corpo (cura), e de dois corpos (transmissão). As transições nada mais são do que regras para configurações de estados de saúde de cada agente, e cada par de agentes, na configuração $|\mu\rangle$. Essas regras são 
armazenadas em tabelas de consulta ou dicionários. A entrada deste dicionário pode ser a configuração a ser testada, como ilustra a Figura 2.4. Cada entrada do dicionário corresponde a uma tuple com dois valores imutáveis, a saber, a acoplamento da transição e a configuração de saída.

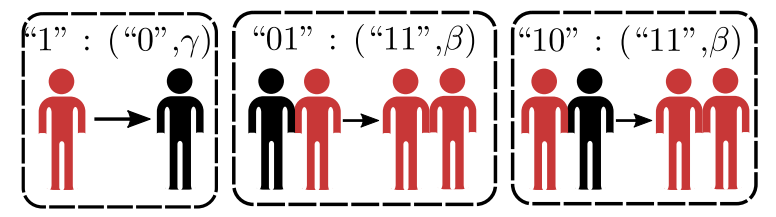

Figura 2.4: Transições não-diagonais para o modelo SIS. A estrutura de dados segue a convenção income-outcome. Cada entrada do dicionário corresponde um tipo de estado de entrada, enquanto o primeiro valor da tuple descreve a configuração de saída.

Com as transições diagonais e fora da diagonal em mãos, basta inspecionar cada agente (pares de agente) e produzir as novas configurações. O Algoritmo 3 mostra as transições não-diagonais de um corpo, enquanto a Figura 2.5 ilustra o procedimento.

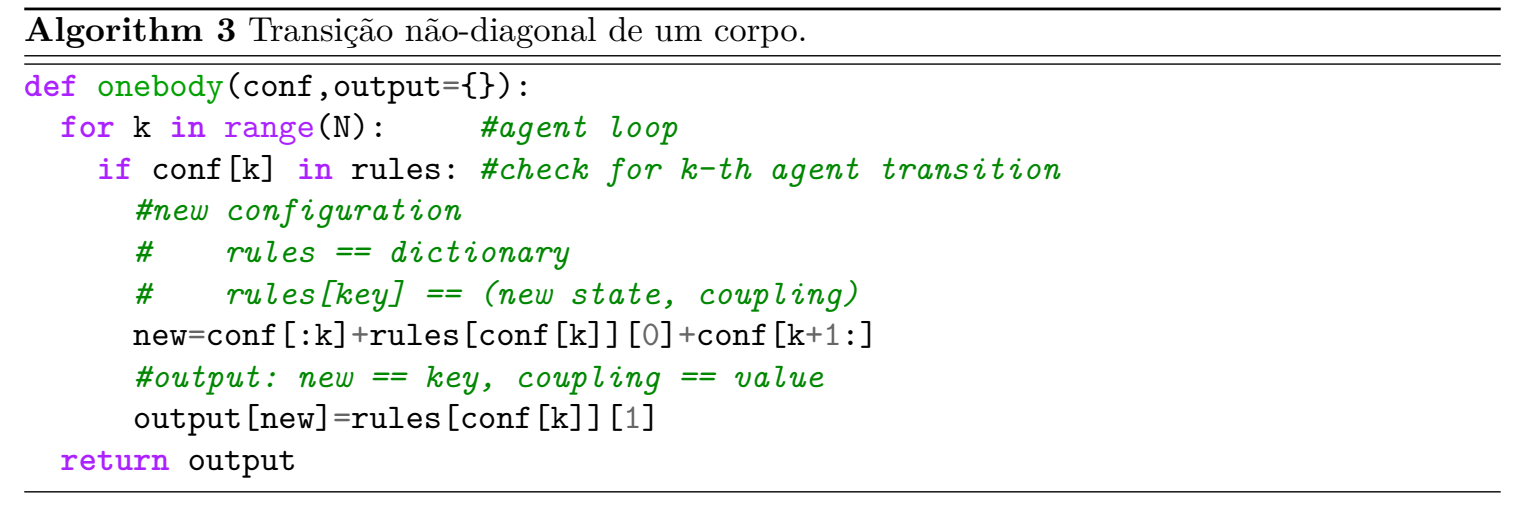

O procedimento para o cálculo das contribuições de dois corpos é semelhante aquele das contribuições de um corpo. Ao invés de percorrer sobre os agentes, fazse a checagem de todos os pares $(i, j)$ de agentes possíveis, e então multiplica-se pelo elemento $A_{i j}$ da matriz de adjacência. Apresentamos um código computacional simplificado no Algoritmo 4, e cuja aplicação é ilustrada na Figura 2.6. 


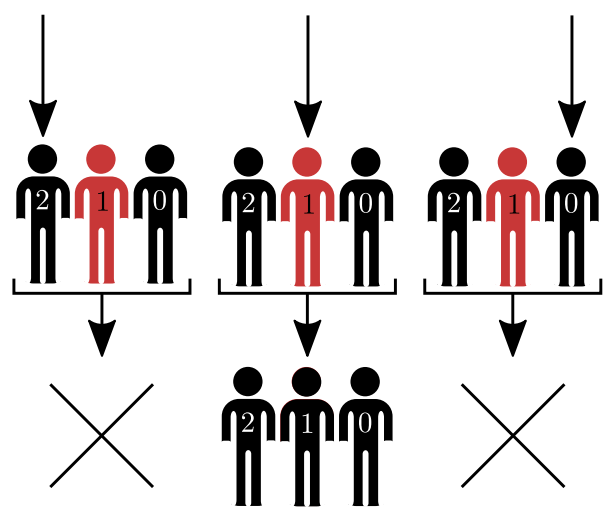

Figura 2.5: Contribuição não-diagonal de um corpo para a configuração $|2\rangle$, no modelo SIS com $N=3$. Apenas o agente infectado em $k=1$ produz transição, resultando na configuração $|0\rangle$.

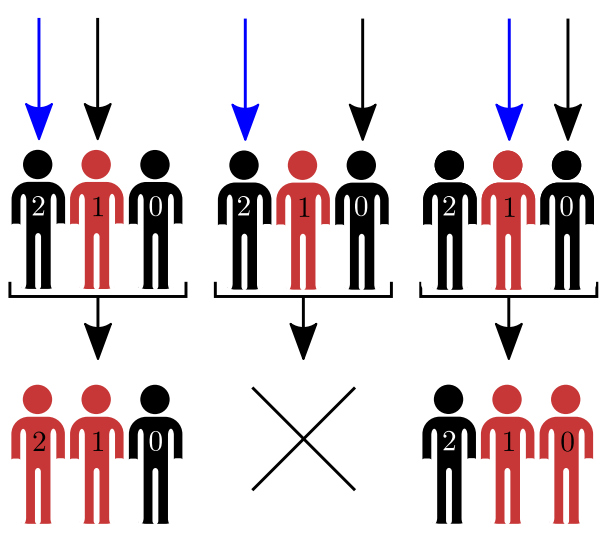

Figura 2.6: Contribuição não-diagonal de dois corpos para a configuração $|2\rangle$, no modelo SIS com $N=3$. Aqui, $A_{i j}=1-\delta_{i j}$. O agente $k=1$ contamina o agente $k=0(k=2)$, cuja configuração resultante é $|3\rangle(|6\rangle)$.

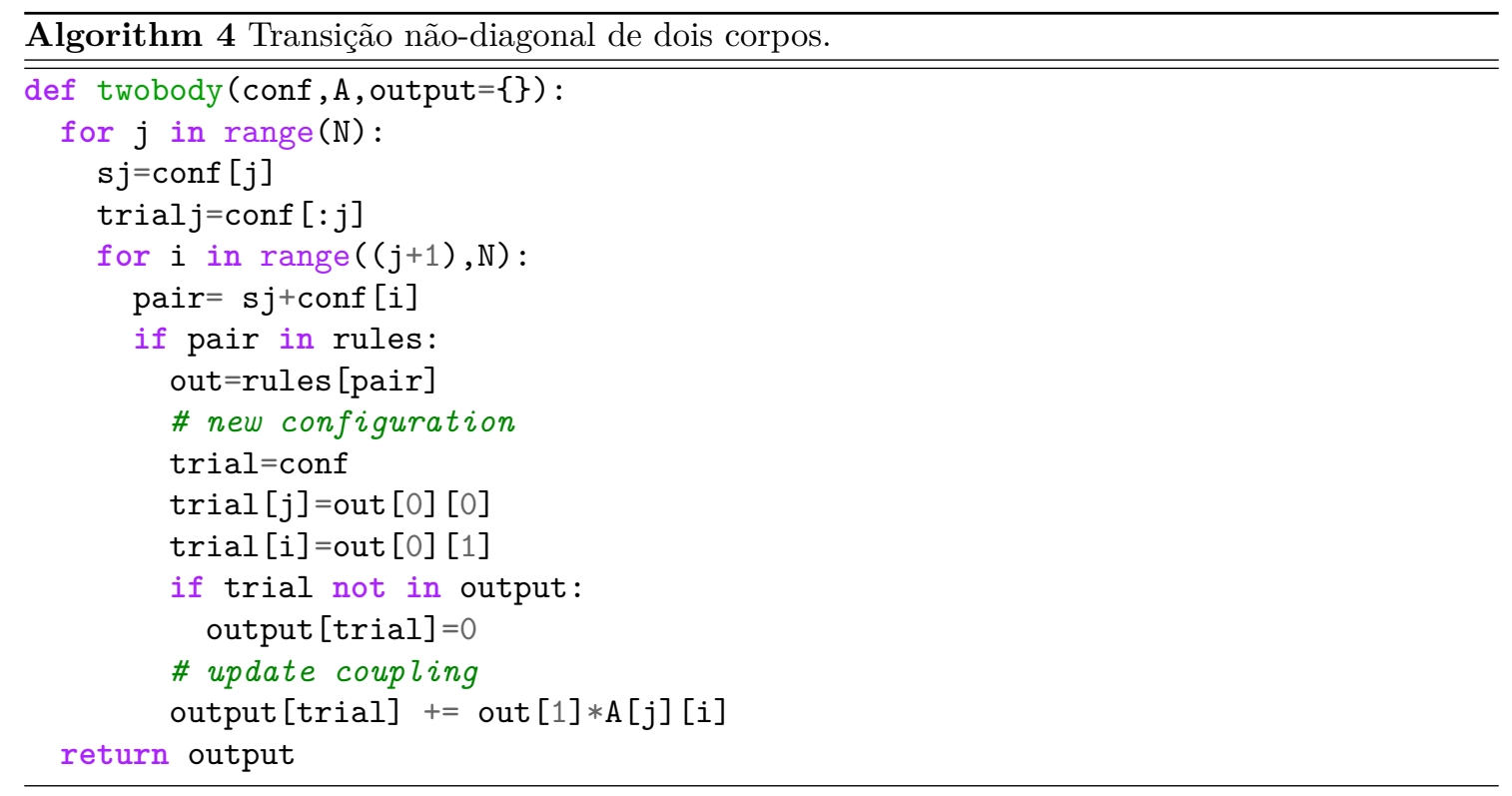




\section{Apêndice}

\section{Matriz de transição para mais que dois estados epidêmicos}

Os conceitos desenvolvidos na seção anterior são facilmente expandidos para a construção da matriz de transição de modelos epidemiológicos com mais de dois estados de saúde. As configurações $\left|C_{\mu}\right\rangle$ que geram o espaço de Hilbert correspondente são aqueles definidas pelas Eqs. (3.18a-3.18c). Assim como no caso $q=2$, a confecção de $\hat{T}$ requer o estudo das transições diagonais e não-diagonais.

Para $q=2$, as transição não-diagonais foram recuperadas por meio dos operadores de levantamento e abaixamento de spin localizado, $\hat{s}_{k}^{ \pm}=\hbar \hat{\sigma}_{k}^{ \pm}$. No entanto, essa escolha não é adequada para $q>2$. A ação dos operadores de levantamento e abaixamento produz

$$
\frac{\hat{s}_{k}^{ \pm}}{\hbar}\left|\sigma_{0} \cdots \sigma_{k} \cdots \sigma_{N-1}\right\rangle=\sqrt{s_{k}\left(s_{k}+1\right)-m_{k}\left(m_{k} \pm 1\right)}\left|\sigma_{0} \cdots\left(\sigma_{k} \pm 1\right) \cdots \sigma_{N-1}\right\rangle,
$$

onde $m_{k}=-s_{k},-s_{k}+1, \ldots, s_{k}$ com os valores de $s_{k}=(q-1) / 2,(q-1) / 2-1, \ldots$, sujeitos à condição $s_{k} \geqslant 0$. O coeficiente escalar $\sqrt{s_{k}\left(s_{k}+1\right)-m_{k}\left(m_{k} \pm 1\right)}$ é o termo problemático aqui, pois especifica o setor de momento angular $s_{k}$. Isto complica significativamente a construção de $\hat{T}$, pois este não pode depender do vetor sobre o qual atua.

\subsection{Matrizes de Weyl}

Uma solução simples para contornar esse problema requer o uso das matrizes $q \times q$ de Weyl [174], $\hat{E}_{k}^{x_{2} x_{1}}, \operatorname{com} k=0,1, \ldots, N-1$ e $x_{1,2}=0,1, \ldots, q-1$. Essas matrizes são bastante simples: todos seus elementos são nulos exceto um, 
$\left(\hat{E}_{k}^{x_{2} x_{1}}\right)_{i j}=\delta_{i, x_{2}} \delta_{j, x_{1}}$. Grosso modo, a matriz $\hat{E}_{k}^{x_{2} x_{1}}$ muda o estado de saúde do $k$-ésimo agente de $x_{1}$ para $x_{2}$, sem a multiplicação por termos adicionais, que é exatamente o comportamento necessário para construir $\hat{T}$.

Assim como no modelo SIS, dividimos as transições em três categorias, a saber, um corpo, dois corpos e diagonais. Transições de um corpo expressam a mudança do estado $x$ para o estado $y$. Correspondem ao produto $D^{y x} \hat{E}_{k}^{y x}$, onde $D^{y x} / \delta t$ é a taxa de transição por unidade de tempo $\delta t$. Analogamente, a transição de dois corpos é dada por $G_{x_{2} x_{1}}^{y_{2} y_{1}} A_{j k} \hat{E}_{j}^{y_{2} y_{1}} \hat{E}_{k}^{x_{2} x_{1}}$, onde $G_{x_{2} x_{1}}^{y_{2} y_{1}} / \delta t$ é a taxa correspondente à transição $\left(y_{1} \rightarrow y_{2}, x_{1} \rightarrow x_{2}\right)$. Nos modelos epidêmicos típicos, o evento de transmissão de patógeno entre agentes geralmente não modifica o estado de saúde do agente previamente infectado, isto é, $x_{1}=x_{2}$.

O mesmo raciocínio empregado para determinar as transições diagonais do modelo SIS podem ser utilizadas aqui. A ideia é determinar a probabilidade de permanecer no estado a partir das possíveis transições não-diagonais. Portanto, a matriz de transição é dada por

$$
\hat{T}=\mathbb{1}-\sum_{k, j=0}^{N-1} \sum_{a, b, c=0}^{q-1} G_{c c}^{b a} A_{j k}\left(\hat{E}_{j}^{a a}-\hat{E}_{j}^{b a}\right) \hat{E}_{k}^{c c}-\sum_{k=0}^{N-1} \sum_{a, b=0}^{q-1} D^{b a}\left(\hat{E}_{k}^{a a}-\hat{E}_{k}^{b a}\right) .
$$

A representação matricial de $\hat{T}$ possui dimensão $q^{N} \times q^{N}$. Podemos utilizar o modelo SEIRS como exemplo. Neste caso, $q=4$ e os estados são organizados na ordem $(R, S, E, I)$. Os acoplamentos de dois corpos são todos nulos exceto por $G_{33}^{21}$, que descreve a transição $I S \rightarrow I E$. Já os acoplamentos não-nulos de um corpo são $D^{32}, D^{03}$ e $D^{10}$, que correspondem às transições $E \rightarrow I, I \rightarrow R$ e $R \rightarrow S$, respectivamente.

\subsection{Movimentos difusivos de patógenos}

Na modelagem baseada em agentes, geralmente associa-se um vértice a um agente, enquanto as arestas associadas ao vértice representam especificamente os contatos do agente. Essa vertente privilegia o estado de saúde dos agentes como variável descritiva.

Porém, também é possível descrever o sistema por meio do estado de desenvolvimento do patógeno. Por exemplo, no modelo SIS, a presença do patógeno significa $\sigma=1$, nulo caso contrário. No modelo SIRS, a impossibilidade de seu 
desenvolvimento no hospedeiro no $k$-ésimo vértice é indicado pela variável $\sigma_{k}=0$, susceptível à infecção como $\sigma_{k}=1$, e já desenvolvido como $\sigma_{k}=2$. A primeira vista, ambas modelagens são equivalentes.

Contudo, é permitido ao patógeno - mas não ao agente - migrar entre os vértices. Esse movimento difusivo, em adição ao mecanismo de infecção, produz espalhamento mais efetivo dos patógenos entre a população. Nesse caso, a matriz de transição é dada por

$$
\hat{T}=\mathbb{1}-\sum_{k=0}^{N-1} \sum_{a, b=0}^{q-1} D^{b a}\left(\hat{E}_{k}^{a a}-\hat{E}_{k}^{b a}\right)-\sum_{k, j=0}^{N-1} \sum_{a b c d=0}^{q-1} A_{j k} G_{d c}^{b a}\left(\hat{E}_{j}^{a a} \hat{E}_{k}^{c c}-\hat{E}_{j}^{b a} \hat{E}_{k}^{d c}\right) .
$$

Como exemplo, considere o modelo SIS. Nesse caso, os termos usuais são $D^{01}=\gamma \delta t$ e $G_{11}^{10}=(\beta / N) \delta t$, enquanto os acoplamentos difusivos não-nulos são $G_{01}^{10}=G_{10}^{01}=\Omega$. Para especializar a discussão em torno da difusão, faça $\beta=\gamma=0$. Por meio das identidades para $q=2, \hat{E}_{k}^{00}=1-\hat{n}_{k}$ e $E_{k}^{11}=\hat{n}_{k}$, temos

$$
\hat{T}=\mathbb{1}-\Omega \sum_{k, j} A_{j k}\left[\left(1-\hat{n}_{j}\right) \hat{n}_{k}+\left(1-\hat{n}_{k}\right) \hat{n}_{j}-\left(\hat{\sigma}_{j}^{+} \hat{\sigma}_{k}^{-}+\hat{\sigma}_{j}^{-} \hat{\sigma}_{k}^{+}\right)\right] .
$$

Se $A_{j k}=\delta_{j, k \pm 1}$ representa um grafo regular periódico, então

$$
\hat{T}=\mathbb{1}+\Omega \sum_{k}\left[4 \hat{n}_{k} \hat{n}_{k+1}+2\left(\hat{\sigma}_{k+1}^{+} \hat{\sigma}_{k}^{-}+\hat{\sigma}_{k+1}^{-} \hat{\sigma}_{k}^{+}\right)-4 \hat{n}_{k}\right] .
$$

A substituição $\hat{n}_{k}=\left(\hat{\sigma}_{k}^{z}+1\right) / 2$ produz

$$
\hat{T}=\mathbb{1}+2 \Omega \sum_{k}\left[2^{-1} \hat{\sigma}_{k}^{z} \hat{\sigma}_{k+1}^{z}+\left(\hat{\sigma}_{k+1}^{+} \hat{\sigma}_{k}^{-}+\hat{\sigma}_{k+1}^{-} \hat{\sigma}_{k}^{+}\right)-\hat{n}_{k}\right]+\Omega N .
$$

O operador entre colchetes nada mais é do que o Hamiltoniano do modelo XXZ ferromagnético com acoplamento axial $\Delta=1 / 2$ e campo magnético $h=\Delta$. O modelo XXZ com $\Delta=1 / 2$ é invariante por transformações conforme, possui invariância $Z(2)$, não possui gap no espectro de energia (excitações sem massa e correlações de longo alcance), e preserva a magnetização total.

\subsection{Endemias}

De acordo com a definição, uma epidemia descreve o aumento significativo de ocorrência de uma doença comunicável numa dada população, num certo intervalo de tempo e região geográfica. A menção à localização geográfica tem grande relevância: dez novos casos de malária na Rússia, durante o inverno, certamente qualificam 
a presença de uma epidemia; ja a incidência de dez novos casos de malária na floresta amazônica, durante o inverno, não representa uma incremento expressivo. O motivo do tratamento diferenciado é que malária é uma doença endêmica para populações tropicais. Uma endemia trata de um processo epidêmico, de uma doença comunicável, que atinge estabilidade numa determinada população, geralmente devido a condições geográficas e climáticas favoráveis [90].

Para fins de modelagem matemática, a definição de endemias recorre à palavra chave: estabilidade. Ela estabelece que há uma distribuição de probabilidade estacionária para o processo epidêmico, parametrizada de acordo com fatores demográficos e geoclimáticos. Esse é o nosso ponto de partida para a discussão abaixo.

Concomitante ao desenvolvimento do processo epidêmico do modelo SIS, reforçamos a relevância do gerador das translações temporais, $\hat{H}$, e da matriz de transição $\hat{T}$. Naturalmente, há uma certa semelhança de $\hat{H}$ com o Hamiltoniano de sistemas mais tradicionais. Essa associação de conceitos, porém, depende da natureza intrínseca do problema em questão. Por exemplo, se o processo Markoviano descreve a evolução de um sistema quântico com Hamiltoniano $\hat{\mathbb{H}}$, as probabilidades de transição entre configurações por são expressas por $T_{\mu \nu}=\delta t\left|\left\langle\phi_{\mu}|\hat{\mathbb{H}}| \phi_{\nu}\right\rangle\right|^{2}$. A partir desta observação conclui-se os valores taxas de transição de $\hat{H}$ são funções dos acoplamentos das interações expressas em $\hat{\mathbb{H}}$. Apesar disto, a ação de ambos operadores sempre coincide, de maneira que podemos relacionar um com o outro.

Agora, qual a vantagem em estabelecer a correspondência entre o gerador $\hat{H}$ e o Hamiltoniano $\mathbb{H}$ ? Se permitido, existem situações nas quais o sistema atinge um regime de equilíbrio e, portanto, possui uma distribuição de probabilidade de equilíbrio. Ao invés de lidar com todos os $N$ agentes, podemos examinar apenas o comportamento de $N^{\prime}$ agente e assumir que os $\left(N-N^{\prime}\right) \gg N^{\prime}$ agentes formam um reservatório térmico. Grosso modo, essa hipótese afirma que a probabilidade conjunta dos $N$ agentes é o produto entre a probabilidade do sistema de $N^{\prime}$ agentes pela probabilidade do sistema de $N-N^{\prime}$ agentes. Uma característica importante do reservatório é que suas propriedades, incluindo aí a energia e entropia, são praticamente independentes dos $N^{\prime}$ agentes. Também decorrem duas consequências importantes. Primeiro, a energia total do sistema é a soma das energias do sistema de $N^{\prime}$ agentes, do reservatório e da interação entre ambos. A troca de energia entre 
os $N^{\prime}$ agentes e o reservatório permite o estabelecimento da distribuição canônica para o sistema de $N^{\prime}$ agentes. Segundo, dado que $N-N^{\prime} \gg N^{\prime}$, a entropia do reservatório é muito maior que a entropia do sistema de $N^{\prime}$ agentes. Portanto, podemos definir uma temperatura característica ao reservatório.

Para prosseguir, precisamos definir as energias do sistema. A partir dos termos diagonais do gerador $\hat{H}$, podemos assumir que as energias do sistema de $N^{\prime}$ agentes e reservatório são, respectivamente,

$$
\begin{aligned}
& E^{\prime}=\frac{\beta}{N} \sum_{i j=0}^{N^{\prime}-1} A_{i j}\left(1-n_{i}\right) n_{j}+\gamma \sum_{i=0}^{N^{\prime}-1} n_{i}, \\
& E=\frac{\beta}{N} \sum_{i j=N^{\prime}}^{N-1} A_{i j}\left(1-n_{i}\right) n_{j}+\gamma \sum_{i=N^{\prime}}^{N-1} n_{i} .
\end{aligned}
$$

A função de partição do reservatório não envolve as transições entre configurações e vale $Z=\sum_{\{E\}} \exp \left(-\beta_{B} E\right)$, em que $\beta_{B}$ é o parâmetro que corresponde ao inverso da temperatura. Formalmente, a Eq.(3.34b) equivale ao modelo de Ising anisotrópico, sujeito a um campo magnético, se fizermos a transformação de variáveis $n_{k}=s_{k}+$ $1 / 2$, com $s_{k}= \pm(1 / 2)$, tal que

$$
E=-\frac{\beta}{N} \sum_{i j=N^{\prime}}^{N-1} A_{i j} s_{i} s_{j}+\gamma \sum_{i=N^{\prime}}^{N-1} s_{i}+\left[\frac{\gamma}{2}\left(N-N^{\prime}\right)+\frac{\beta}{4 N} \sum_{i j=N^{\prime}}^{N-1} A_{i j}\right] .
$$

A parcela entre colchetes é apenas uma constante que depende do número de vértices e do grau médio do grafo, ambos restritos ao reservatório. Para $\beta=0$, a menor energia representa o caso onde todos os $N-N^{\prime}$ agentes estão saudáveis. Para $\beta>0$, há uma competição ordem-desordem e, portanto, transição de fase crítica. ${ }^{6}$ De acordo, $\langle s\rangle_{R}=-\left(1 / \beta_{B} N\right)(\partial / \partial \gamma) \ln Z$ expressa o valor médio do spin do reservatório, enquanto a susceptibilidade magnética é dada por $\chi_{R}=(\partial / \partial \gamma)\langle s\rangle_{R}$.

Com a caracterização do reservatório em mãos, podemos examinar o que acontece com sistema de $N^{\prime}$ agentes quando entra em contato com o reservatório. Para simplificar ainda mais a discussão, vamos adotar $N^{\prime}=1$, que contém apenas o agente no vértice $V_{0}$. Nesse caso, existem apenas duas configurações possíveis $|0\rangle$

\footnotetext{
${ }^{6}$ As propriedades do regime crítico dependem da matriz $A$. A dimensão crítica superior do modelo de Ising é $d_{c}=4$ para redes regulares. Isto quer dizer que qualquer sistema descrito pela interação do modelo de Ising, que ocorre numa rede regular com dimensionalidade espacial $d \geqslant 4$, possui comportamento crítico descrito integralmente por uma teoria de campo médio. Aqui, a matriz $A$ pode apresentar regularidade e interação entre vizinhos próximos mas raramente é o caso. Para ao menos estabelecer uma analogia, digamos que a dimensão característica é metade do grau médio do grafo correspondente ao reservatório, $d_{R}=(1 / 2)\langle\kappa\rangle_{R}$. Logo, se $\langle\kappa\rangle_{R} \geqslant 8$, espera-se uma boa descrição do comportamento crítico pela teoria de campo médio (apenas do comportamento crítico).
} 
e $|1\rangle$. A equação mestra para a ocupação média de infectados fica

$$
\frac{d n}{d t}=\frac{\beta}{N} \sum_{k=0}^{N-1} A_{k 0}[1-n(t)]\langle n\rangle_{R}-\gamma n(t)
$$

Definindo-se $\gamma^{\prime} \equiv \beta\langle n\rangle_{R} \kappa_{0} / N$, onde $\kappa_{0}$ é o grau do vértice $V_{0}$, obtém-se a seguinte solução para a Eq. (3.36):

$$
n(t)=n(0) \mathrm{e}^{-\left(\gamma+\gamma^{\prime}\right) t}+\left(\frac{\gamma^{\prime}}{\gamma+\gamma^{\prime}}\right)\left[1-\mathrm{e}^{-\left(\gamma+\gamma^{\prime}\right) t}\right] .
$$

Portanto, a densidade de equilíbrio é $n_{e}=\gamma^{\prime} /\left(\gamma+\gamma^{\prime}\right)$.

Vejamos em detalhes dois limites interessantes para a solução da Eq. (3.37), para baixas temperaturas do reservatório. Para o caso $\gamma / \beta^{\prime} \ll 1$ e grafo completo, há quebra espontânea de simetria e o reservatório encontra-se completamente infectado ou completamente susceptível. Considere o primeiro cenário, cujo valor esperado $\langle n\rangle_{R}=1-\varepsilon$ onde $\varepsilon \ll 1$. Logo, $n_{e}=(1-\gamma / \beta)+o(\gamma \varepsilon / \beta)$, que é o resultado esperado do modelo compartimental. Considere agora o caso contrário, isto é, $\gamma^{\prime} / \gamma \ll 1$, cujo resultado é $n_{e}=\beta\langle n\rangle_{R} / \gamma+o\left(\left(\gamma^{\prime} / \gamma\right)^{2}\right)$.

Não se deve esperar que a investigação de um único agente seja capaz de reproduzir a dinâmica de uma doença endêmica com precisão. O motivo é que o processo epidêmico do sistema de $N^{\prime}$ agentes não possui qualquer heterogeneidade, mesmo que as propriedades do reservatório sejam bastante representativas. Portanto, deve-se considerar um valor $N^{\prime} \geqslant 1$ a fim de se capturar aspectos oriundos da heterogeneidade da população. 


\section{Apêndice}

\section{4}

\section{Aproximações}

\subsection{Estados coerentes}

Simetrias são cruciais na redução da complexidade de operadores de muitos corpos. Além das simetrias associadas à topologia da matriz de adjacência, seja de um grafo ou de uma rede, existem também as simetrias internas, características dos estados de saúde dos agentes, e aquelas derivadas dos processos de interação.

Para grafos completos, a simetria por permutações arbitrárias garante a conservação de um número "quântico" $j$. Tanto a notação quanto a descrição sugerem a interpretação de $j$ como o valor do momento angular total. Claro, trata-se somente de uma interpretação. Podemos levar essa interpretação adiante e estudar seus efeitos e metodologias que são tipicamente associadas a ela.

Dito isto, por que estudar metodologias associadas à teoria do momento angular de muitos corpos ao invés de tratar com ela diretamente? A resposta, muito embora nãos seja excitante, é que a construção dos estados $|j, m\rangle$ em termos de um conjunto de $N$ variáveis torna-se exaustivamente complexa para $N \gg 1$. Não somente complexo, mas muitas vezes gera confusão. Por exemplo, considere um sistema formado por $N=3$ partículas de spin $s=1 / 2$. Este sistema conta com $2^{3}=8$ configurações distintas. E também possui 8 estados na base $|j, m\rangle$. Como é bem sabido, $j=1 / 2(m= \pm 1 / 2)$ ou $j=3 / 2(m= \pm 3 / 2, \pm 1 / 2)$, que à primeira vista totalizam 6 estados. O problema, que na verdade não é problemático, se origina na degenerescência dos estados $j=1 / 2$ e $m= \pm 1 / 2$, pois cada estado possui duas representações em diferentes setores de permutação cíclica (Apêndice 2). Mais especificamente, considere as combinações lineares de vetores com $m=-1 / 2$ : 


$$
\frac{|100\rangle+\mathrm{e}^{2 \imath \pi p / 3}|010\rangle+\mathrm{e}^{4 \imath \pi p / 3}|001\rangle}{\sqrt{3}},
$$

onde o inteiro $p$ pode assumir os valores 0,1 e 2 . Quando $p=0$, reconhecemos essa combinação linear por $|j=3 / 2, m=-1 / 2\rangle$. Ora, então segue que ambas as combinações remanescentes são válidas para descrever o par $(j=1 / 2, m=-1 / 2)$.

Uma forma muito simples de escapar do dilema envolvendo a multiplicidade dos vetores $|j, m\rangle$ tem origem na teoria do momento angular posto adiante por Schwinger [19]. A ideia consiste em utilizar os autovetores e operadores do oscilador harmônico, que são muito mais simples, ao invés de trabalhar com os autovetores e operadores do momento angular. Essa associação não é exata, restrita a alguns setores de $j$ e setores de permutação. Contudo, quando disponível, o operador resultante cai na mesma classe de problemas típicos estudados em Ótica Quântica e alguns sistemas ferromagnéticos.

O primeiro passo para estabelecer a conexão com os operadores $\hat{a}$ e $\hat{a}^{\dagger}$ do oscilador harmônico, tal que $\left[\hat{a}, \hat{a}^{\dagger}\right]=\mathbb{1}$, fundamenta-se na transformação de HolsteinPrimakoff [11]. Essa transformação foi introduzida por Primakoff e Holstain nos anos 40 para descrever ondas de magnetização, também conhecidas por spin waves. No contexto presente e para o setor de momento angular $j$, as transformações são

$$
\begin{aligned}
& \hat{J}^{+}=\sqrt{2 j+1-\hat{n}} \hat{a}^{\dagger}, \\
& \hat{J}^{-}=\hat{a} \sqrt{2 j+1-\hat{n}} .
\end{aligned}
$$

Essa formulação é escolhida de modo a garantir as relações de comutação entre os operadores $\hat{J}^{ \pm, z}$. Se mantidos dentro da raiz quadrada, estes operadores são muito complicados. Porém, se a ação destes operadores restringe-se apenas a vetores com $n \ll(2 j+1)$, então a raiz pode ser linearizada. Essa condição tem mais chance de ser satisfeita para $j=N / 2$. Neste setor, temos

$$
\begin{aligned}
& \hat{J}^{+}=\sqrt{N+1-\hat{n}} \hat{a}^{\dagger}, \\
& \hat{J}^{-}=\hat{a} \sqrt{N+1-\hat{n}},
\end{aligned}
$$

os quais sob a condição $\langle n\rangle / N \ll 1$ são linearizados:

$$
\hat{J}^{+} \approx \sqrt{N+1}\left(1-\frac{1}{2} \frac{\hat{a}^{\dagger} \hat{a}}{N+1}\right) \hat{a}^{\dagger},
$$




$$
\hat{J}^{-} \approx \hat{a} \sqrt{N+1}\left(1-\frac{1}{2} \frac{\hat{a}^{\dagger} \hat{a}}{N+1}\right) \quad .
$$

No que segue, utilizamos apenas o espaço vetorial correspondente ao oscilador harmônico [41], cujos autovetores são $|m\rangle(m=0,1,2, \ldots)$ com $\hat{a}|0\rangle=0$. Nessa "mudança" de base, o espaço vetorial deixa de ter dimensão finita (momento angular) e passa a ter infinitos estados (oscilador). O truque agora consiste em especificar a propriedade principal do tipo das soluções desejadas, em concordância com o vínculo $|n\rangle \ll N$. Em particular, podemos procurar soluções que sejam robustas frente a atuação de operadores $\hat{a}$ e $\hat{a}^{\dagger}$. Esse tipo de estado é compatível com o que se espera do sistema no regime de equilíbrio.

Como ansatz podemos considerar que tais estados são estados coerentes [85]. Estados coerentes $\lambda$ são combinações lineares dos estados o oscilador harmônico,

$$
|\lambda\rangle=\mathrm{e}^{-\lambda^{2} / 2} \sum_{m=0}^{\infty} \frac{\lambda^{m}}{\sqrt{m !}}|m\rangle,
$$

onde aqui escolhemos $\lambda \in \mathbb{R}$. É fácil ver que $\langle\lambda \mid \lambda\rangle=1$. A propriedade mais importante dos estados coerentes é que esle são autovetores do operador não-hermitiano $\hat{a}$, ou seja,

$$
\hat{a}|\lambda\rangle=\lambda|\lambda\rangle
$$

Essa estado possui tantos quanta que a remoção de um único quantum não altera o perfil do sistema. Por este motivo, encontra uma fértil área de atuação em Ótica Quântica, embora seja necessário contabilizar efeitos de decoerência e grandes perdas, etc. Para nós, a equação de autovalor provê $\langle\lambda|\hat{a}| \lambda\rangle=\left\langle\lambda\left|\hat{a}^{\dagger}\right| \lambda\right\rangle=\lambda \mathrm{e}$ $\left\langle\lambda\left|\hat{a}^{\dagger} \hat{a}\right| \lambda\right\rangle=\lambda^{2}$.

Antes de prosseguir convém calcular duas propriedades. A primeira estabelece a igualdade $\hat{n}^{2}=\hat{a}^{\dagger} \hat{a}^{\dagger} \hat{a} \hat{a}+\hat{a}^{\dagger} \hat{a}$. A segunda é obtida a partir das Eqs. (4.41a) e (4.41b):

$$
\left\langle\lambda\left|\hat{J}^{+} \hat{n}\right| \lambda\right\rangle=\left\langle\lambda\left|\hat{n} \hat{J}^{-}\right| \lambda\right\rangle=\sqrt{N+1} \lambda^{3}\left[\left(1-\frac{1}{N+1}\right)-\frac{\lambda^{2}}{2 N+2}\right] .
$$

A substituição da Eq. (4.44) na Eq.(3.76) produz seguinte o valor esperado para estados coerentes:

$$
\frac{1}{N^{2}} \frac{\langle\lambda|\hat{\mathcal{H}}| \lambda\rangle}{\beta / N} \approx \frac{\lambda^{2}}{N}\left(1+\frac{\Gamma}{N}-\frac{\lambda^{2}}{N}\right)-\frac{1}{2} \frac{\lambda}{\sqrt{N}}\left(\frac{\lambda^{2}}{N}+\frac{\Gamma}{N}\right)\left(2-\frac{\lambda^{2}}{N}\right)+o\left(\frac{\lambda^{3}}{N^{5 / 2}}\right) .
$$




\subsection{Aproximação de Bethe-Peierls}

Via de regra, perturbações para a topologia de redes não são obrigadas a afetar todas as arestas de mesma maneira. Quando isso acontece, amplia-se a significância da contribuição de campo médio, como vimos na seção anterior. O mesmo raciocínio se aplica ao operador não-perturbado. Aqui apresentamos um método baseado na aproximação de Bethe-Peierls [118] para estimar os autovetores e autovalores $\Lambda_{\mu}$, bem como as correções perturbativas $\Delta \Lambda_{\mu}^{(1)}$.

A aproximação de Bethe-Peierls é mais conhecida em Mecânica Estatística como aproximação de campo médio. Nessa aproximação, substitui-se um dos operadores diagonais de termos quadráticos por operadores lineares. Por meio desta substituição, tenta-se expressar o valor típico do operador diagonal localizado $\hat{n}_{k} \mathrm{em}$ termos de seu valor médio global $\bar{n}$, isto é, $\hat{O}_{j} \hat{n}_{k} \approx \hat{O}_{j} \bar{n}$, onde $\hat{O}_{j}$ é um operador localizado arbitrário.

Os operadores da Eq. (3.74b) são lineares e, portanto, permanecem inalterados. Já os operadores da Eq. (3.74a) são modificados de maneira que temos

$$
\hat{\mathcal{H}} \approx \frac{\beta}{2 N}\left[N \Gamma+\bar{n} \sum_{j} \kappa_{j}+2 \sum_{j}\left(\Gamma-\bar{n} \kappa_{j}\right) \hat{\sigma}_{j}^{z}-2 \sum_{j}\left(\Gamma+\bar{n} \kappa_{j}\right) \hat{\sigma}_{j}^{x}\right],
$$

com $\kappa_{j}=\sum_{k} A_{j k}$ e $\left[\hat{\sigma}_{k}^{a}, \hat{\sigma}_{k^{\prime}}^{b}\right]=\imath \delta_{k, k^{\prime}} \sum_{c=x, y, z} \epsilon^{a b c} \hat{\sigma}_{k}^{c}$. Nesse ponto, convém fazer a identificação

$$
\begin{aligned}
\theta_{j} & =\tan ^{-1}\left(\frac{\Gamma+\bar{n} \kappa_{j}}{\Gamma-\bar{n} \kappa_{j}}\right), \\
\Omega^{\prime} & =\Gamma N+\bar{n} \sum_{j} \kappa_{j}, \\
\Omega_{j} & =\sqrt{2\left(\Gamma^{2}+\bar{n}^{2} \kappa_{j}^{2}\right)}, \\
\hat{\mathcal{H}} & \approx \frac{\beta}{2 N}\left[\Omega^{\prime}+2 \sum_{j} \Omega_{j}\left(\cos \theta_{j} \hat{\sigma}_{j}^{z}-\sin \theta_{j} \hat{\sigma}_{j}^{x}\right)\right],
\end{aligned}
$$

O operador da Eq. (4.47d) trata de $N$ operadores separáveis, os quais são diagonalizáveis por meio de rotações ao redor do eixo $y$, isto é, $\hat{U}_{k}=\exp \left(-\imath \theta_{k} \hat{\sigma}_{k}^{y}\right)$. Portanto, os autovalores aproximados são

$$
\Lambda_{\mu}=\frac{\beta}{2 N}\left[\Omega^{\prime}+\sum_{j} \Omega_{j} \mathrm{e}^{\imath \pi \ell_{j}},\right]
$$

onde os números $\ell_{j}= \pm 1$ para $j=0,1, \ldots, N-1$. 


\section{Apêndice}

\section{Aplicações em processos estocásticos com memória}

A examinação de processos estocásticos por meio das propriedades espectrais da matriz de transição $\hat{T}$ revela informações relevantes quanto aos estados estacionários. Tradicionalmente, o foco da análise espectral centra-se sobre o gerador temporal $\hat{H}=(\mathbb{1}-\hat{T}) / \delta t$. Ao longo do Capítulo 3, mostramos que os estados estacionários, bem com o ponto de máxima entropia durante o regime transiente, podem ser obtidos a partir da Eq. (3.65), que utiliza o gerador simétrico $\hat{\mathcal{H}}=(1 / 2)\left(\hat{H}+\hat{H}^{\dagger}\right)$. A escolha de uma metodologia em detrimento da outra depende tão somente das simetrias do gerador correspondente - e suas vantagens - na resolução das equações espectrais.

Amarrando todas essas metodologias para processos estocásticos, encontrase a linearidade da matriz de transição, que por sua vez é consequência natural apenas para processos Markovianos. Essa restrição não é pequena: ela impõe que a evolução temporal de eventos dependem única e exclusivamente do estado atual do sistema. De fato, essa definição contempla problemas importantes para o entendimento de processos físicos básicos como movimento de partículas em meios difusivos, transições atômicas, etc. Contudo, essa definição também exclui uma série de processos estocásticos onde decisões ou eventos prévios influenciam a evolução temporal corrente. Essas situações são típicas nas decisões, comportamento e aprendizado humano ou animal, por exemplo. Isso também vem recebendo mais importância no cenário tecnológico atual com o desenvolvimento automatizado de extração de padrões e classificadores de objetos. 
Porém, há uma pequena classe de processos estocásticos com memória (processos não-Markovianos) que podem ser transformados em processos Markovianos com a introdução adequada de graus de liberdade. Por falta de nome mais adequado, denominamos esse procedimento como transmutação Markoviana. Aqui, vamos examinar como se dá essa transformação, bem como o significado dos graus de liberdade adicionais, para o exemplo da caminhada do turista [175]. ${ }^{7}$ É importante frisar que após a transmutação o processo estocástico pode ser resolvido por meio da análise espectral.

Em sua versão original, a caminhada do turista descreve um viajante que sempre visita o vértice (sítio) mais próximo não visitado nos últimos $\mu$ passos. Nesta formulação, existem dois vínculos: a visitação do vértice mais próximo, segundo alguma métrica (por exemplo, distância espacial euclideana); a exclusão das últimas $\mu$ localidades visitadas. Este último vínculo fornece o caráter não-Markoviano do problema, ao contrário da visitação determinística de sítios mais próximos. Aqui, adotamos a generalização da Ref. [176] e relaxamos a condição determinística de visitação do vizinho mais próximo, portanto, permitindo saltos.

A transmutação desse problema exige que seja introduzidos $\mu$ graus de liberdade adicionais, como mostramos a seguir. Como de praxe, a caminhada se dá ao longo de um grafo, cuja matriz de adjacência $A$ possui dimensão $N \times N$. Também é necessário definir a matriz de vizinhos $D(N \times N)$ descreve a ordem relativa dos vértices em relação à distância, isto é, a ordem do vizinho. Para uma dada coluna $k, D_{k k}=0$, pois o vizinho de ordem 0 é o próprio vértice; enquanto o vértice $j$ corresponde ao vizinho de ordem $m, D_{j k}=m$, em que $m \leqslant N-1 .^{8}$

O "truque" da transmutação consiste em definir $\mu+1$ variedades ${ }^{9}$ de viajantes. Dessa maneira, se o viajante da variedade $s>0$ sempre se mover para a posição do viajante da variedade $s-1$, a sucessão de eventos entre os $\mu+1$ viajantes reproduz a trilha de visitação do viajante da variedade 0 (o viajante real). Formalmente, o

\footnotetext{
${ }^{7}$ Um outro processo estocástico com memória passível de transmutação Markoviana é a caminhada aleatória auto-repulsiva. Neste problema, cada vértice é identificado unicamente por um número inteiro $k=0,1, \ldots, N-1$, com matriz de adjacência $A$. Na versão mais simples do problema, cada vértice comporta no máximo uma partícula de dois tipos (sabores) diferentes. Os operadores de criação e destruição localizados correspondentes são $\hat{\sigma}_{s k}^{+}$, onde o índice $s=0,1$ representa a variedade da partícula. Os vetores de base são $\left|n_{00} n_{10} ; n_{01} n_{11} ; \cdots\right\rangle$ com $n_{s k}=0,1$. A matriz de transição deste problema é: $\hat{T}=\sum_{k j} \hat{\sigma}_{1 k}^{+} \hat{\sigma}_{0 k}^{+} \hat{\sigma}_{0 j}^{-} \hat{p}_{k j}$. O operador $\hat{p}_{k j}=A_{k j} /\left[\sum_{m} A_{m j}\left(\mathbb{1}-\hat{\sigma}_{1 m}^{+} \hat{\sigma}_{1 m}^{-}\right)\right]$ determina as probabilidades condicionais.

${ }^{8}$ A desigualdade $m \leqslant N-1$ surge se permitirmos que dois vértices sejam vizinhos de mesma ordem em relação a algum $k$. Isso ocorre quando as distâncias são iguais para uma dada precisão nas medidas de distância.

${ }^{9}$ Nota: Admito que neste trecho, a influência das nomenclaturas da Cromodinâmica quase venceu à sobriedade geralmente esperada em Mecânica Estatística. No fim, mantive o adjetivo variedades ao invés de sabores.
} 
espaço de Hilbert correspondente requer vetores com $\mu+1$ graus de liberdade por vértice,

$$
\left|\sigma_{00} \sigma_{10} \cdots \sigma_{\mu 0} ; \sigma_{01} \sigma_{11} \cdots \sigma_{\mu 1} ; \cdots ; \sigma_{0 N-1} \sigma_{1 N-1} \cdots \sigma_{\mu N-1}\right\rangle
$$

em que $\sigma_{r k}=0$ ou 1 e, portanto, possui dimensão $2^{N(\mu+1)}$. Na prática, a simetria que expressa a conservação do número de viajantes (um para cada variedade) reduz o número de estados disponíveis para $N^{\mu+1}$.

Em seguida, considere os os operadores localizados de levantamento $\left(\hat{\sigma}_{s k}^{+}\right)$, abaixamento $\left(\hat{\sigma}_{s k}^{-}\right)$e número $\left(\hat{n}_{s k}=\hat{\sigma}_{s k}^{+} \hat{\sigma}_{s k}^{-}\right)$para o viajante de variedade $s$ no vértice $k$. Esses operadores são necessários para descrever o movimento dos viajantes pelo grafo. Aliás, dentre os movimentos disponíveis, os movimentos mais simples são aqueles que expressam a memória, ou seja, o viajante de variedade $s$ move-se do vértice $j$ para o vértice $k$, correspondente à posição do viajante de variedade $s-1$, $\hat{\sigma}_{s k}^{+} \hat{\sigma}_{s j}^{+}$. Contudo, o $s$-ésimo viajante precisa confirmar que a variedade $s-1$ está de fato em $k$. Isto é realizado por meio do operador número $\hat{n}_{s-1 k}, \operatorname{logo}\left(\hat{\sigma}_{s k}^{+} \hat{n}_{s-1 k}\right) \hat{\sigma}_{s j}^{-}$ reproduz o movimento desejado para $s>0$.

O movimento do viajante original (variedade $s=0$ ) carrega em si a maior parte da complexidade do problema. Em parte isto se dá pois ele é quem determina o movimento de todos os outros. Além disso, não visita um vértice que já contém ao menos um viajante de qualquer espécie. De fato, expressa-se seu movimento da seguinte maneira: $\left(\hat{\sigma}_{0 k}^{+} \hat{\sigma}_{0 j}^{-}\right) \hat{p}_{k j}$. A parcela entre parênteses descreve o movimento uniforme enquanto $\hat{p}_{k j}$ determina a probabilidade do movimento acontecer na direção dos vértices $j$ para $k$ com $j \neq k$. Podemos inferir que os autovalores deste operador são proporcionais aos elementos de matriz de $A_{j k}$ e a $\exp \left[-\beta D_{k j} f(n)\right]$, em que $\beta$ é um parâmetro e $f(n)$ é uma função monotônica crescente. O parâmetro $\beta$ desempenha o papel de inverso de temperatura, tal que $\beta \gg 1$ tende a selecionar caminhos com $D_{k j}=1$. Como ansatz, escolhemos $f(n)=1+\chi n$, onde o parâmetro $\chi$ sempre respeita o vínculo $\beta \chi \gg 1$. Assim, a probabilidade associada ao caminho $j \rightarrow k$ é

$$
\begin{aligned}
p_{k j} & =\frac{1}{Z_{j}} A_{k j} \exp \left[-\beta D_{k j}\left(1+\chi \sum_{s=0}^{\mu+1} n_{s k}\right)\right], \\
Z_{j} & =\sum_{i=0}^{N-1} A_{i j} \exp \left[-\beta D_{i j}\left(1+\chi \sum_{s=0}^{\mu+1} n_{s i}\right)\right] .
\end{aligned}
$$


A fim de permitir saltos entre vértices distantes $\left(D_{k j}>1\right)$ como alternativa à visitação de vértices já ocupados, devemos também impor o vínculo $\exp (-\beta \chi) / \exp (-\beta N) \ll 1, \log 0 \chi \gg$. Sob tais condições, o viajante tente a visitar localidades distantes para evitar dupla visitação. Destaca-se que, em princípio, o formalismo acima permite a visitação de contendo número não nulo de visitantes, embora com probabilidade proporcional a $\mathrm{e}^{-\beta \chi D_{k j}}$. Em contrapartida, não é necessário lidar com probabilidades condicionais, o que por si só facilita aplicações e estabelece a conexão com a generalização proposta na Ref. [176].

Em resumo, a matriz de transição do sistema $\hat{T} \equiv \hat{T}_{0} \hat{T}_{1} \cdots \hat{T}_{\mu}$ nada mais é do que a composição de $\mu+1$ operadores ordenados, tal que

$$
\begin{aligned}
\hat{T}_{s>0} & =\sum_{k j=0}^{N-1}\left(\hat{\sigma}_{s k}^{+} \hat{n}_{s-1 k}\right) \hat{\sigma}_{s j}^{-}, \\
\hat{T}_{0} & =\sum_{k j=0}^{N-1}\left(\hat{\sigma}_{0 k}^{+} \hat{\sigma}_{0 j}^{-}\right) \hat{p}_{k j}, \\
\hat{p}_{k j} & =\frac{1}{Z_{j}} A_{k j} \exp \left[-\beta D_{k j}\left(1+\chi \sum_{s=0}^{\mu+1} \hat{n}_{s k}\right)\right] .
\end{aligned}
$$

A condição inicial estabelece que todos os viajantes partem de um mesmo vértice no instante de tempo $t=0$. Esse problema também contempla uma espécie de estacionariedade, onde o viajante fica preso em caminhos cíclicos, semelhante ao conceito de localização. Um caminho cíclico de $\ell$ passos satisfaz à equação de autovalor

$$
\hat{T}^{\ell}|\lambda\rangle=|\lambda\rangle
$$

Portanto, os autovalores associados à rota cíclica valem $\mathrm{e}^{2 \imath \pi m / \ell} \operatorname{com} m=0,1 \ldots, \ell-$ 1. Já os autovetores dependem explicitamente das matrizes $A$ e $D$, mais especificamente, subregiões do grafo, cuja dinâmica (do turista) é invariante por permutações finitas. Esse problema espectral é bastante fértil e encontra aplicações em áreas diversas como processamento de imagens. 


\section{Referências $^{10}$}

[1] R. Pastor-Satorras e A. Vespignani, "Epidemic dynamics and endemic states in complex networks", Phys. Rev. E, vol. 63, p. 066117, May 2001. 1, 39, 62

[2] A. G. Haldane e R. M. May, "Systemic risk in banking ecosystems", Nature, vol. 469, pp. 351-355, Jan 2011. 1

[3] R. M. May, S. A. Levin e G. Sugihara, "Complex systems: Ecology for bankers", Nature, vol. 451, pp. 893-895, Feb 2008. 1

[4] E. Bullmore e O. Sporns, "Complex brain networks: graph theoretical analysis of structural and functional systems", Nat. Rev. Neurosci., vol. 10, pp. 186198, Mar 2009. 1

[5] F. Iglói, R. Juhász e H. Rieger, "Random antiferromagnetic quantum spin chains: Exact results from scaling of rare regions", Phys. Rev. B, vol. 61, pp. 11552-11568, May 2000. 1

[6] E. Dagotto, "Complexity in strongly correlated electronic systems", Science, vol. 309, no. 5732, pp. 257-262, 2005. 1

[7] F. Dyson, "Statistical theory of the energy levels of complex system I", J. Math. Phys, vol. 3, 1962.

[8] F. Dyson, "Statistical theory of the energy levels of complex system II", J. Math. Phys, vol. 3, 1962.

[9] F. Dyson, "Statistical theory of the energy levels of complex system III", $J$. Math. Phys, vol. 3, 1962. 1

\footnotetext{
${ }^{10}$ De acordo com o estilo LMSC (baseado no estilo IEEE Transactions - Electrical and Electronics Engineers).
} 
[10] E. Lieb, T. Schultz e D. Mattis, "Two soluble models of an antiferromagnetic chain", Ann. Phys. (N.Y.), vol. 16, no. 3, pp. 407 - 466, 1961. 2, 15, 52, 53, 113

[11] T. Holstein e H. Primakoff, "Field dependence of the intrinsic domain magnetization of a ferromagnet", Phys. Rev., vol. 58, pp. 1098-1113, Dec 1940. 2, $23,100,154$

[12] N. N. Bogoljubov, "On a new method in the theory of superconductivity", Nuovo Cimento, vol. 7, no. 6, pp. 794-805, 1958. 2, 123

[13] M. Keeling e K. Eames, "Networks and epidemic models", J. R. Soc. Interface, vol. 2, no. 4, pp. 295-307, 2005. 2, 38, 39, 45, 46, 131

[14] A.-L. Barabási e R. Albert, "Emergence of scaling in random networks", Science, vol. 286, no. 5439, pp. 509-512, 1999. 2, 12, 46

[15] M. Tinkham, Group theory and quantum mechanics. Courier Corporation, 2003. $2,6,7,100$

[16] E. H. Stanley, Introduction to phase transition and critical phenomena. New York: Oxford University Press, 1979. 2, 20, 83

[17] J. B. Kogut, "An introduction to lattice gauge theory and spin systems", Rev. Mod. Phys., vol. 51, pp. 659-713, Oct 1979. 2, 14, 53, 72, 110

[18] E. Ising, "Beitrag zur theorie des ferromagnetismus", Z. Physik, vol. 31, 1925. 2

[19] J. Schwinger, On angular momentum. Jan 1952. 2, 23, 154

[20] L. Reichl, A modern course in statistical physics. Wiley, 1998. 3, 11, 43, 50, 57

[21] R. Pastor-Satorras, C. Castellano, P. Van Mieghem e A. Vespignani, "Epidemic processes in complex networks", Rev. Mod. Phys., vol. 87, pp. 925-979, Aug 2015. $3,36,37,39,46,51,62$

[22] H. Heesterbeek, R. M. Anderson, V. Andreasen, S. Bansal, D. De Angelis, C. Dye, K. T. D. Eames, W. J. Edmunds, S. D. W. Frost, S. Funk, T. D. 
Hollingsworth, T. House, V. Isham, P. Klepac, J. Lessler, J. O. Lloyd-Smith, C. J. E. Metcalf, D. Mollison, L. Pellis, J. R. C. Pulliam, M. G. Roberts e C. Viboud, "Modeling infectious disease dynamics in the complex landscape of global health", Science, vol. 347, no. 6227, 2015. 3, 43

[23] S. Bansal, B. T. Grenfell e L. A. Meyers, "When individual behaviour matters: homogeneous and network models in epidemiology", J. R. Soc. Interface, vol. 4, no. 16, pp. 879-891, 2007. 3, 38, 39, 46

[24] R. H. Dicke, "Coherence in spontaneous radiation processes", Phys. Rev., vol. 93, pp. 99-110, Jan 1954. 3, 14, 86, 87, 88

[25] M. Tavis e F. W. Cummings, "Exact solution for an $n$-molecule-radiationfield hamiltonian", Phys. Rev., vol. 170, pp. 379-384, Jun 1968. 14, 87

[26] K. Hepp e E. H. Lieb, "On the superradiant phase transition for molecules in a quantized radiation field: the Dicke maser model", Ann. Phys. (NY), vol. 76, no. 2 , pp. $360-404,1973.3,87$

[27] J. G. Bohnet, Z. Chen, J. M. Weiner, D. Meiser, M. J. Holland e J. K. Thompson, "A steady-state superradiant laser with less than one intracavity photon", Nature (London), vol. 484, pp. 78-81, Apr 2012. 3, 101

[28] D. Meiser e M. J. Holland, "Intensity fluctuations in steady-state superradiance", Phys. Rev. A, vol. 81, p. 063827, Jun 2010. 101

[29] G. Khitrova e H. M. Gibbs, "Quantum dots: Collective radiance", Nat. Phys., vol. 3 , no. 2, pp. $84-86,2007.3$

[30] W. Heisenberg, "Zur theorie des ferromagnetismus", Z. Phys., vol. 49, no. 9, pp. 619-636, 1928. 4, 110

[31] F. Alcaraz, M. Droz, M. Henkel e V. Rittenberg, "Reaction-diffusion processes, critical dynamics, and quantum chains", Ann. Phys., vol. 230, no. 2, pp. 250 $-302,1994.4$

[32] M. Henkel, Conformal Invariance and Critical Phenomena. Heidelberg: Springer, 1999. 4, 20, 63, 72, 112 
[33] J. L. Cardy, Phase Transition and Critical Phenomena, vol. 11. New York: Academic Press, 1987. 4, 20, 63

[34] F. C. Alcaraz e G. M. Nakamura, "Phase diagram and spectral properties of a new exactly integrable spin-1 quantum chain", J. Phys. A: Math. Gen., vol. 43 , no. 15 , p. 155002 , 2010. 4, 12, 14, 17, 20, 114

[35] H. W. J. Blöte, J. L. Cardy e M. P. Nightingale, "Conformal invariance, the central charge, and universal finite-size amplitudes at criticality", Phys. Rev. Lett., vol. 56, pp. 742-745, Feb 1986. 112

[36] J. L. Cardy, "Conformal invariance and universality in finite-size scaling", J. Phys. A: Math. Gen., vol. 17, no. 7, 1984. 20

[37] J. L. Cardy, "Conformal invariance and surface critical behavior", Nucl. Phys. $B$, vol. 240 , no. $4,1984.4$

[38] H. Goldstein, Classical mechanics. Addison-Wesley, 1950. 5, 6, 71

[39] L. D. Landau e E. M. Lifshitz, Mechanics: Volume 1 (Course of Theoretical Physics). Butterworth-Heinemann, 2nd ed. ed., 1982. 5, 7, 71

[40] A. Messiah, Quantum Mechanics. Dover, 1999. 5, 8, 48, 74

[41] J. J. Sakurai e S. F. Tuan, Modern quantum mechanics. Addison-Wesley, 1994. $5,7,11,19,20,64,76,99,155$

[42] L. D. Landau e E. M. Lifshitz, Quantum Mechanics: Non-Relativistic Theory. Butterworth-Heinemann, 3rd ed. ed., 2003. 5, 7, 48, 74, 88

[43] M. Hamermesh, Group theory and its application to physical problems. Courier Corporation, 1962. 6, 139

[44] E. Noether, "Invariante variationsprobleme", Nachrichten von der Gesellschaft der Wissenschaften zu Göttingen, Mathematisch-Physikalische Klasse, vol. 1918, pp. 235-257, 1918. 6

[45] E. Noether, "Invariant variation problems", Transport Theory and Statistical Physics, vol. 1, no. 3, pp. 186-207, 1971. 6 
[46] G. E. Uhlenbeck e S. Goudsmit, "Spinning electrons and the structure of spectra", Nature (London), vol. 117, pp. 264-265, fev. 1926. 8

[47] A. Pais, "George Uhlenbeck and the discovery of electron spin", Phys. Today, vol. 42, pp. 34-40, dez. 1989. 8

[48] W. Pauli, "The connection between spin and statistics", Phys. Rev., vol. 58, pp. 716-722, Oct 1940. 11, 14

[49] W. Pauli, "Über den zusammenhang des abschlusses der elektronengruppen im atom mit der komplexstruktur der spektren", Z. Phys., vol. 31, no. 1, pp. 765-783, 1925. 11

[50] F. J. Dyson e A. Lenard, "Stability of matter. I", J. Math. Phys., vol. 8, no. 3, pp. $423-434,1967.11$

[51] N. Ashcroft e N. D. Mermin, Solid state physics. Saunders College, 1988. 12, 20,45

[52] R. Baxter, Exactly soluble models in statistical mechanics. New York: Academic Press, 1990. 14, 78

[53] F. Reif, Fundamentals of statistical and thermal physics. Waveland Press, 2009. 20, 43, 72, 134, 135

[54] D. J. Amit e V. Martín-Mayor, Field Theory, the Renormalization Group, and Critical Phenomena: Graphs to Computers. World Scientific Press, jun. 2005. $12,14,20,63,72$

[55] R. Albert e A.-L. Barabási, "Statistical mechanics of complex networks", Rev. Mod. Phys., vol. 74, pp. 47-97, Jan 2002. 12, 45, 46, 133

[56] A. Barrat, M. Barthélemy, R. Pastor-Satorras e A. Vespignani, "The architecture of complex weighted networks", Proc. Natl. Acad. Sci. USA, vol. 101, no. 11 , pp. $3747-3752,2004.12$

[57] A. Arenas, A. Díaz-Guilera e C. J. Pérez-Vicente, "Synchronization reveals topological scales in complex networks", Phys. Rev. Lett., vol. 96, p. 114102, Mar 2006. 
[58] M. Boguñá e R. Pastor-Satorras, "Epidemic spreading in correlated complex networks", Phys. Rev. E, vol. 66, p. 047104, Oct 2002. 39, 62

[59] T. Aoki e T. Aoyagi, "Scale-free structures emerging from co-evolution of a network and the distribution of a diffusive resource on it", Phys. Rev. Lett., vol. 109, p. 208702, Nov 2012. 12

[60] E. T. Jaynes e F. W. Cummings, "Comparison of quantum and semiclassical radiation theories with application to the beam maser", Proc. IEEE, vol. 51, pp. 89-109, Jan 1963. 14, 87

[61] C. Emary e T. Brandes, "Chaos and the quantum phase transition in the dicke model", Phys. Rev. E, vol. 67, p. 066203, Jun 2003. 91

[62] J. Keeling, "Coulomb interactions, gauge invariance, and phase transitions of the Dicke model", J. Phys.: Condens. Matter, vol. 19, no. 29, p. 295213, 2007.

[63] P. W. Milonni, J. R. Ackerhalt e H. W. Galbraith, "Chaos in the semiclassical $n$-atom jaynes-Cummings model: Failure of the rotating-wave approximation", Phys. Rev. Lett., vol. 50, pp. 966-969, Mar 1983.

[64] L. Amico e K. Hikami, "Integrable spin-boson interaction in the TavisCummings model from a generic boundary twist", Eur. Phys. J. B, vol. 43, no. 3, pp. 387-391, 2005.

[65] M. D. Crisp, "Jaynes-Cummings model without the rotating-wave approximation", Phys. Rev. A, vol. 43, pp. 2430-2435, Mar 1991. 14

[66] G. M. Nakamura, M. Mulato e A. S. Martinez, "Spin gap in coupled magnetic layers", Physica A, vol. 451, pp. 313 - 319, 2016. 14, 124

[67] E. Dagotto e T. M. Rice, "Surprises on the way from one- to two-dimensional quantum magnets: The ladder materials", Science, vol. 271, no. 5249, pp. 618 623, 1996.

[68] I. Affleck, "Universal term in the free energy at a critical point and the conformal anomaly", Phys. Rev. Lett., vol. 56, pp. 746-748, Feb 1986. 20 
[69] I. Affleck, T. Kennedy, E. Lieb e H. Tasaki, "Rigorous results on valence-bond ground states in antiferromagnets", Phys. Rev. Lett., vol. 59, pp. 799-802, Aug 1987.

[70] I. Affleck, "Quantum spin chains and the haldane gap", J. Phys.: Condens. Matter., vol. 1, no. 19, p. 3047, 1989.

[71] F. C. Alcaraz e A. L. Malvezzi, "Conformal invariance and the phase diagram of the anisotropic heisenberg spin-1 ladder", J. Phys.: Condens. Matter., vol. 9, no. 14 , p. $3055,1997.14$

[72] G. M. Nakamura, "Cadeias quânticas de spin : alguns estudos numéricos e analíticos", Dissertação de Mestrado, Universidade de São Paulo, Instituto de Física de São Carlos, 2006. 15

[73] A. Tsvelik, "Field theory for a fermionic ladder with generic intrachain interactions", Phys. Rev. B, vol. 83, p. 104405, Mar 2011. 15

[74] H. Bethe, "Zur theorie der metalle", Z. Phys., vol. 71, no. 3-4, pp. 205-226, 1931. $17,78,114,140$

[75] H. Frahm e A. Schadschneider, "Critical exponents of the degenerate hubbard model", J. Phys. A: Math. Gen., vol. 26, no. 7, p. 1463, 1993.

[76] F. C. Alcaraz, M. N. Barber e M. T. Batchelor, "Conformal invariance, the xxz chain and the operator content of two-dimensional critical systems", Ann. Phys., vol. 182, no. 2, pp. 280 - 343, 1988. 17, 20

[77] J. J. Sakurai, Advanced quantum mechanics. Addison-Wesley, 1967. 19, 74, 87,88

[78] J. L. Cardy, "Operator content of two-dimensional conformally invariant theories", Nucl. Phys. B, vol. 270, no. 0, 1986. 20

[79] J. J. Binney, N. J. Dowrick, A. J. Fisher e M. Newman, The theory of critical phenomena: an introduction to the renormalization group. Oxford University Press, Inc., 1992. 20, 72

[80] F. Woynarovich, "Excitation spectrum of the spin- $1 / 2$ heisenberg chain and conformal invariance", Phys. Rev. Lett., vol. 59, pp. 259-261, Jul 1987. 20 
[81] F. C. Alcaraz, M. N. Barber e M. T. Batchelor, "Conformal invariance, the xxz chain and the operator content of two-dimensional critical systems", Ann. Phys., vol. 182, 1988. 20

[82] K. G. Wilson, "Renormalization group and critical phenomena. i. renormalization group and the kadanoff's scaling picture.", Phys. Rev. B, vol. 4, 1971. 20,83

[83] A. Belavin, A. Polyakov e A. Zamolodchikov, "Infinite conformal symmetry in two-dimensional quantum field theory", Nucl. Phys. B, vol. 241, no. 2, pp. 333 $-380,1984.20$

[84] V. Knizhnik e A. Zamolodchikov, "Current algebra and Wess-Zumino model in two dimensions", Nucl. Phys. B, vol. 247, no. 1, pp. 83 - 103, 1984. 20

[85] R. J. Glauber, "Coherent and incoherent states of the radiation field", Phys. Rev., vol. 131, pp. 2766-2788, Sep 1963. 26, 155

[86] G. M. Nakamura, A. C. P. Monteiro, G. C. Cardoso e A. S. Martinez, "Efficient method for comprehensive computation of agent-level epidemic dissemination in networks", Scientific Reports, vol. 7, p. 40885, 1 2017. 29, 47, 56, 66, 68, 70

[87] G. M. Nakamura, A. C. P. Monteiro, G. C. Cardoso e A. S. Martinez, "Finite symmetries in epidemic models", ArXiv e-prints, set. 2016. 29

[88] J. M. Last, J. H. Abramson e G. D. Freidman, A dictionary of epidemiology, vol. 141. Oxford Univ. Press, 2001. 30

[89] R. Pastor-Satorras e A. Vespignani, "Epidemic spreading in scale-free networks", Phys. Rev. Lett., vol. 86, pp. 3200-3203, Apr 2001. 30, 39, 62, 80

[90] R. Bonita, R. Beaglehole e T. Kjellström, Basic epidemiology. World Health Organization, 2006. 30, 31, 34, 150

[91] W. O. Kermack e A. G. McKendrick, "A contribution to the mathematical theory of epidemics", Proc. R. Soc. A, vol. 115, no. 772, pp. 700-721, 1927. 30

[92] S. Riley, C. Fraser, C. A. Donnelly, A. C. Ghani, L. J. Abu-Raddad, A. J. Hedley, G. M. Leung, L.-M. Ho, T.-H. Lam, T. Q. Thach, P. Chau, K.-P. 
Chan, S.-V. Lo, P.-Y. Leung, T. Tsang, W. Ho, K.-H. Lee, E. M. C. Lau, N. M. Ferguson e R. M. Anderson, "Transmission dynamics of the etiological agent of sars in hong kong: Impact of public health interventions", Science, vol. 300, no. 5627, pp. 1961-1966, 2003. 31

[93] J. Heffernan, R. Smith e L. Wahl, "Perspectives on the basic reproductive ratio", J. R. Soc. Interface, vol. 2, no. 4, pp. 281-293, 2005. 31

[94] G. I. S. Network(GISN), "Organização mundial de saúde/flunet”, 2016. último accesso em 31.12.2016. 33

[95] W. E. R. Team, "West african ebola epidemic after one year — slowing but not yet under control", N. Engl. J. Med., vol. 372, no. 6, pp. 584-587, 2015. 32

[96] G. D. Maganga, J. Kapetshi, N. Berthet, B. Kebela Ilunga, F. Kabange, P. Mbala Kingebeni, V. Mondonge, J.-J. T. Muyembe, E. Bertherat, S. Briand, J. Cabore, A. Epelboin, P. Formenty, G. Kobinger, L. González-Angulo, I. Labouba, J.-C. Manuguerra, J.-M. Okwo-Bele, C. Dye e E. M. Leroy, "Ebola virus disease in the democratic republic of congo", N. Engl. J. Med., vol. 371, no. 22, pp. 2083-2091, 2014. 32

[97] M. D. Van Kerkhove, A. I. Bento, H. L. Mills, N. M. Ferguson e C. A. Donnelly, "A review of epidemiological parameters from ebola outbreaks to inform early public health decision-making”, Sci. Data, vol. 2, pp. 150019 EP -, May 2015. Data Descriptor. 32

[98] J. Mlakar, M. Korva, N. Tul, M. Popović, M. Poljšak-Prijatelj, J. Mraz, M. Kolenc, K. Resman Rus, T. Vesnaver Vipotnik, V. Fabjan Vodušek, A. Vizjak, J. Pižem, M. Petrovec e T. Avšič Županc, "Zika virus associated with microcephaly", N. Engl. J. Med., vol. 374, no. 10, pp. 951-958, 2016. 32

[99] M. E. J. Newman, "The structure and function of complex networks", SIAM Review, vol. 45, no. 2, pp. 167-256, 2003. 37, 46, 133

[100] K. T. D. Eames e M. J. Keeling, "Modeling dynamic and network heterogeneities in the spread of sexually transmitted diseases", Proc. Natl. Acad. Sci. USA, vol. 99, no. 20, pp. 13330-13335, 2002. 39, 43, 45 
[101] S. Gómez, A. Arenas, J. Borge-Holthoefer, S. Meloni e Y. Moreno, "Discretetime markov chain approach to contact-based disease spreading in complex networks", EPL (Europhysics Letters), vol. 89, no. 3, p. 38009, 2010. 39

[102] R. Pathria, Statistical Mechanics (Second Edition). Oxford: ButterworthHeinemann, second edition ed., 1996. 43, 63

[103] N. G. van Kampen, Stochastic Processes in Physics and Chemistry, vol. 1. Elsevier Science, Amsterdam, 1992. 43, 51

[104] S. Boccaletti, V. Latora, Y. Moreno, M. Chavez e D.-U. Hwang, "Complex networks: Structure and dynamics", Phys. Rep., vol. 424, no. 4-5, pp. $175-$ 308, 2006. 45, 46

[105] F. Chung e L. Lu, "The average distances in random graphs with given expected degrees", Proc. Natl. Acad. Sci. USA, vol. 99, no. 25, pp. 15879-15882, 2002.

[106] R. Durrett, "Some features of the spread of epidemics and information on a random graph", Proc. Natl. Acad. Sci. USA, vol. 107, no. 10, pp. 4491-4498, 2010. $45,55,76$

[107] R. Albert, H. Jeong e A.-L. Barabasi, "Error and attack tolerance of complex networks", Nature, vol. 406, pp. 378-382, Jul 2000. 46

[108] D. J. Watts e S. H. Strogatz, "Collective dynamics of small-world networks", Nature, vol. 393, pp. 440-442, Jun 1998. 46, 78

[109] S. Boccaletti, G. Bianconi, R. Criado, C. del Genio, J. Gómez-Gardeñes, M. Romance, I. Sendiña-Nadal, Z. Wang e M. Zanin, "The structure and dynamics of multilayer networks", Phys. Rep., vol. 544, no. 1, pp. $1-122$, 2014. The structure and dynamics of multilayer networks. 46

[110] C. D. Batista e G. Ortiz, "Generalized jordan-wigner transformations", Phys. Rev. Lett., vol. 86, pp. 1082-1085, Feb 2001. 53

[111] W. H. Press, Numerical recipes 3rd edition: The art of scientific computing. Cambridge University Press, 2007. 58 
[112] P. V. Mieghem, J. Omic e R. Kooij, "Virus spread in networks", IEEE/ACM Transactions on Networking, vol. 17, pp. 1-14, Feb 2009. 62

[113] P. Van Mieghem, "The n-intertwined sis epidemic network model", Computing, vol. 93, no. 2, pp. 147-169, 2011. 63

[114] P. Van Mieghem, D. Stevanović, F. Kuipers, C. Li, R. van de Bovenkamp, D. Liu e H. Wang, "Decreasing the spectral radius of a graph by link removals", Phys. Rev. E, vol. 84, p. 016101, Jul 2011.

[115] P. Van Mieghem e E. Cator, "Epidemics in networks with nodal self-infection and the epidemic threshold", Phys. Rev. E, vol. 86, p. 016116, Jul 2012.

[116] E. Cator e P. Van Mieghem, "Second-order mean-field susceptible-infectedsusceptible epidemic threshold”, Phys. Rev. E, vol. 85, p. 056111, May 2012.

[117] H. Wang, Q. Li, G. D'Agostino, S. Havlin, H. E. Stanley e P. Van Mieghem, "Effect of the interconnected network structure on the epidemic threshold", Phys. Rev. E, vol. 88, p. 022801, Aug 2013. 62, 63

[118] H. A. Bethe, "Statistical theory of superlattices", Proc. R. Soc. A, vol. 150, no. 871 , pp. $552-575,1935.63,156$

[119] W. Kohn e L. J. Sham, "Self-consistent equations including exchange and correlation effects", Phys. Rev., vol. 140, pp. A1133-A1138, Nov 1965. 65

[120] P. Hohenberg e W. Kohn, "Inhomogeneous electron gas", Phys. Rev., vol. 136, pp. B864-B871, Nov 1964. 65

[121] J. W. Eaton, D. Bateman, S. Hauberg e R. Wehbring, GNU Octave version 3.8.1 manual: a high-level interactive language for numerical computations. CreateSpace Independent Publishing Platform, 2014. ISBN 1441413006. 70

[122] R. P. Feynman, "Space-time approach to non-relativistic quantum mechanics", Rev. Mod. Phys., vol. 20, pp. 367-387, Apr 1948. 71

[123] H. J. Rothe, Lattice gauge theories: an introduction, vol. 74. World Scientific, 2005. 72 
[124] A. Parvan e T. Biró, "Extensive rényi statistics from non-extensive entropy", Phys. Lett. A, vol. 340, no. 5-6, pp. 375 - 387, 2005. 72

[125] C. Tsallis, "Possible generalization of boltzmann-gibbs statistics", J. Stat. Phys., vol. 52, no. 1, pp. 479-487, 1988. 73

[126] R. Courant e D. Hilbert, Methods of Mathematical Physics, vol. 1. Interscience, 1953. 76

[127] E. Fermi, "Quantum theory of radiation", Rev. Mod. Phys., vol. 4, pp. 87-132, Jan 1932. 85

[128] H. A. Bethe, "The electromagnetic shift of energy levels", Phys. Rev., vol. 72, pp. 339-341, Aug 1947. 85

[129] M. Kozub, L. Pawicki e P. Machnikowski, "Enhanced spontaneous optical emission from inhomogeneous ensembles of quantum dots is induced by shortrange coupling", Phys. Rev. B, vol. 86, p. 121305, Sep 2012. 86

[130] M. Scheibner, T. Schmidt, L. Worschech, A. Forchel, G. Bacher, T. Passow e D. Hommel, "Superradiance of quantum dots", Nat. Phys., vol. 3, pp. 106-110, Feb 2007.

[131] V. I. Yukalov e E. P. Yukalova, "Dynamics of quantum dot superradiance", Phys. Rev. B, vol. 81, p. 075308, Feb 2010. 86, 87

[132] V. N. Pustovit e T. V. Shahbazyan, "Cooperative emission of light by an ensemble of dipoles near a metal nanoparticle: The plasmonic Dicke effect", Phys. Rev. Lett., vol. 102, p. 077401, Feb 2009. 86

[133] T. V. Teperik e A. Degiron, "Superradiant optical emitters coupled to an array of nanosize metallic antennas", Phys. Rev. Lett., vol. 108, p. 147401, Apr 2012. 86

[134] V. I. Yukalov e E. P. Yukalova, "Possibility of superradiance by magnetic nanoclusters", Laser Physics Letters, vol. 8, no. 11, p. 804, 2011. 86

[135] P. Rotondo, M. Cosentino Lagomarsino e G. Viola, "Dicke simulators with emergent collective quantum computational abilities", Phys. Rev. Lett., vol. 114, p. 143601, Apr 2015. 86 
[136] A. T. Sornborger, A. N. Cleland e M. R. Geller, "Superconducting phase qubit coupled to a nanomechanical resonator: Beyond the rotating-wave approximation", Phys. Rev. A, vol. 70, p. 052315, Nov 2004. 86, 88

[137] S. Hofferberth, B. Fischer, T. Schumm, J. Schmiedmayer e I. Lesanovsky, "Ultracold atoms in radio-frequency dressed potentials beyond the rotatingwave approximation", Phys. Rev. A, vol. 76, p. 013401, Jul 2007. 86

[138] M. P. Baden, K. J. Arnold, A. L. Grimsmo, S. Parkins e M. D. Barrett, "Realization of the Dicke model using cavity-assisted Raman transitions", Phys. Rev. Lett., vol. 113, p. 020408, Jul 2014. 86

[139] M. Gross e S. Haroche, "Superradiance: An essay on the theory of collective spontaneous emission", Phys. Rep., vol. 93, no. 5, pp. $301-396,1982.87$

[140] Y. Kaluzny, P. Goy, M. Gross, J. M. Raimond e S. Haroche, "Observation of self-induced Rabi oscillations in two-level atoms excited inside a resonant cavity: The ringing regime of superradiance", Phys. Rev. Lett., vol. 51, pp. 11751178, Sep 1983. 87

[141] N. Skribanowitz, I. P. Herman, J. C. MacGillivray e M. S. Feld, "Observation of Dicke superradiance in optically pumped HF gas", Phys. Rev. Lett., vol. 30, pp. 309-312, Feb 1973. 87

[142] J. A. Mlynek, A. A. Abdumalikov, C. Eichler e A. Wallraff, "Observation of Dicke superradiance for two artificial atoms in a cavity with high decay rate", Nat. Commun., vol. 5, Nov 2014. Article. 87, 101

[143] R. Bonifacio, P. Schwendimann e F. Haake, "Quantum statistical theory of superradiance. i", Phys. Rev. A, vol. 4, pp. 302-313, Jul 1971. 87

[144] P. L. Knight e L. Allen, "Rotating-wave approximation in coherent interactions", Phys. Rev. A, vol. 7, pp. 368-370, Jan 1973. 87

[145] J. Jing, Z.-G. Lü e Z. Ficek, "Breakdown of the rotating-wave approximation in the description of entanglement of spin-anticorrelated states", Phys. Rev. A, vol. 79, p. 044305, Apr 2009. 87 
[146] C. Emary e T. Brandes, "Quantum chaos triggered by precursors of a quantum phase transition: The Dicke model", Phys. Rev. Lett., vol. 90, p. 044101, Jan 2003. 88,101

[147] T. Brandes, "Excited-state quantum phase transitions in Dicke superradiance models", Phys. Rev. E, vol. 88, p. 032133, Sep 2013. 88, 101

[148] Q.-H. Chen, Y.-Y. Zhang, T. Liu e K.-L. Wang, "Numerically exact solution to the finite-size Dicke model", Phys. Rev. A, vol. 78, p. 051801, Nov 2008. 88

[149] E. K. Irish, "Generalized rotating-wave approximation for arbitrarily large coupling", Phys. Rev. Lett., vol. 99, p. 173601, Oct 2007. 88

[150] H. Zheng, S. Y. Zhu e M. S. Zubairy, "Quantum Zeno and anti-Zeno effects: Without the rotating-wave approximation", Phys. Rev. Lett., vol. 101, p. 200404, Nov 2008.

[151] M. O. Scully, "Collective Lamb shift in single photon Dicke superradiance", Phys. Rev. Lett., vol. 102, p. 143601, Apr 2009.

[152] Y. Li, J. Evers, W. Feng e S.-Y. Zhu, "Spectrum of collective spontaneous emission beyond the rotating-wave approximation", Phys. Rev. A, vol. 87, p. 053837, May 2013. 88

[153] T. Werlang, A. V. Dodonov, E. I. Duzzioni e C. J. Villas-Bôas, "Rabi model beyond the rotating-wave approximation: Generation of photons from vacuum through decoherence", Phys. Rev. A, vol. 78, p. 053805, Nov 2008. 88

[154] K. Baumann, C. Guerlin, F. Brennecke e T. Esslinger, "Dicke quantum phase transition with a superfluid gas in an optical cavity", Nature (London), vol. 464, pp. 1301-1306, Apr 2010. 90

[155] D. Meiser, J. Ye, D. R. Carlson e M. J. Holland, "Prospects for a millihertzlinewidth laser", Phys. Rev. Lett., vol. 102, p. 163601, Apr 2009. 101

[156] Y. Chen, Z. Yu e H. Zhai, "Superradiance of degenerate fermi gases in a cavity", Phys. Rev. Lett., vol. 112, p. 143004, Apr 2014. 101 
[157] J. Keeling, M. J. Bhaseen e B. D. Simons, "Fermionic superradiance in a transversely pumped optical cavity", Phys. Rev. Lett., vol. 112, p. 143002, Apr 2014. 101

[158] A. P. Alivisatos, "Semiconductor clusters, nanocrystals, and quantum dots", Science, vol. 271, no. 5251, pp. 933-937, 1996. 107

[159] A. Henglein, "Small-particle research: physicochemical properties of extremely small colloidal metal and semiconductor particles", Chem. Rev., vol. 89, no. 8, pp. 1861-1873, 1989. 107

[160] R. H. Kodama, "Magnetic nanoparticles", J. Magn. Magn. Mater., vol. 200, no. 1 , pp. 359-372, 1999. 107

[161] R. C. Ashoori, H. L. Stormer, J. S. Weiner, L. N. Pfeiffer, K. W. Baldwin e $\mathrm{K}$. W. West, " $N$-electron ground state energies of a quantum dot in magnetic field", Phys. Rev. Lett., vol. 71, pp. 613-616, Jul 1993. 107

[162] J. Dorantes-Dávila e G. M. Pastor, "Magnetic anisotropy of one-dimensional nanostructures of transition metals", Phys. Rev. Lett., vol. 81, pp. 208-211, Jul 1998. 108

[163] P. Gambardella, A. Dallmeyer, K. Maiti, M. Malagoli, W. Eberhardt, K. Kern e C. Carbone, "Ferromagnetism in one-dimensional monatomic metal chains", Nature (London), vol. 416, no. 6878, pp. 301-304, 2002. 108

[164] G. H. Wannier, "The structure of electronic excitation levels in insulating crystals", Phys. Rev., vol. 52, pp. 191-197, Aug 1937. 109

[165] J. des Cloizeaux e J. J. Pearson, "Spin-wave spectrum of the antiferromagnetic linear chain", Phys. Rev., vol. 128, pp. 2131-2135, Dec 1962. 111

[166] S. Owerre e M. Paranjape, "Macroscopic quantum spin tunneling with two interacting spins", Phys. Rev. B, vol. 88, p. 220403, Dec 2013. 111, 115

[167] S. Murakami, N. Nagaosa e S.-C. Zhang, "Dissipationless quantum spin current at room temperature", Science, vol. 301, no. 5638, pp. 1348-1351, 2003. 111 
[168] P. Paluskar, J. Attema, G. de Wijs, S. Fiddy, E. Snoeck, J. Kohlhepp, H. Swagten, R. de Groot e B. Koopmans, "Spin tunneling in junctions with disordered ferromagnets", Phys. Rev. Lett., vol. 100, p. 057205, Feb 2008.

[169] M. L. Polianski e P. W. Brouwer, "Current-induced transverse spin-wave instability in a thin nanomagnet", Phys. Rev. Lett., vol. 92, p. 026602, Jan 2004. 111

[170] S. G. Tan, M. B. A. Jalil, S. B. Kumar e G.-C. Liang, "Spin tunneling in multilayer spintronic devices", Phys. Rev. B, vol. 77, p. 085424, Feb 2008. 115

[171] J. Bardeen, L. N. Cooper e J. R. Schrieffer, "Theory of superconductivity", Phys. Rev., vol. 108, pp. 1175-1204, Dec 1957. 118

[172] M. B. Hastings, I. González, A. B. Kallin e R. G. Melko, "Measuring renyi entanglement entropy in quantum monte carlo simulations", Phys. Rev. Lett., vol. 104, p. 157201, Apr 2010. 129

[173] P. O. B. Netto, Grafos: teoria, modelos, algoritmos. Edgard Blücher, 2003. 131

[174] F. C. Alcaraz e M. J. Lazo, "Exact solutions of exactly integrable quantum chains by a matrix product ansatz", J. Phys. A: Math. Gen., vol. 37, no. 14, p. $4149,2004.147$

[175] G. F. Lima, A. S. Martinez e O. Kinouchi, "Deterministic walks in random media", Phys. Rev. Lett., vol. 87, p. 010603, Jun 2001. 158

[176] J. M. S. Berbert, "Transição entre os comportamentos estendido e localizado em caminhadas estocásticas parcialmente auto-repulsivas em sistemas desordenados unidimensionais", Dissertação de Mestrado, Universidade de São Paulo, Ribeirão Preto, 2009. 158, 160 